$71-17,987$

DIGGES, Kennerly Hite, 1933-

THEORY OF AN AIR CUSHION LANDING SYSTEM FOR AIRCRAFT.

The Ohio State University, Ph.D., 1970

Engineering, mechanical

University Microfilms, A XEROX Company , Ann Arbor, Michigan

(c) Copyright by

Kennerly Ftite Digges

1971 


\section{THEORY OF AN AIR CUSHION \\ LANDING SYSTEM FOR AIRCRAFT}

\section{DISSEFTATION}

Presented in Partial Fulfillment of the Requirements for the Degree of Doctor of Philosophy in the Graduate

School of The Ohio State University

By

KENNERLYH. DIGGES, B.S., M.Sc.

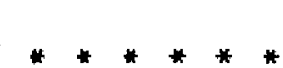

The Ohio State University

1970

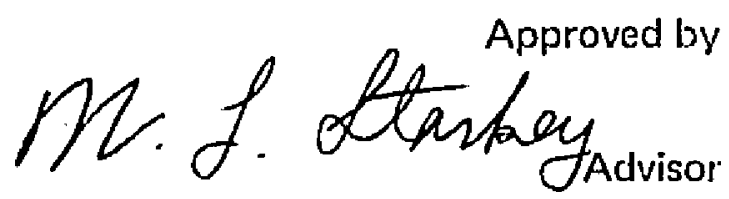

Department of Mechanical Engineering 
PLEASE NOTE :

Some pages have small and indistinct type.

Filmed as received.

University Microfilms 


\section{ACKNOWLEDGEMENTS}

The aluthor wishes to express his appreciation for the contributions of a number of persons who greatly assisted in this work. For their valuable contributions in conducting tests, plotting curves, and making computations the efforts of the following personnel were noteworthy: B. J. Brookman, G. R. Wyan, D. M. Gorman, J. E. Krysiak, D. J. Perez, S. Campluell, D. J. Pool, and B. K. Wansgard. The expertise of S. Lanaccia and K. Johnson in developing workable computer programs was a substantial contribution to the success of this work. For her excellence in grammatical corrections and in the final typing of the manuscript, the contribution of Mary Brooks is gratefully acknowledged.

Fin.lly, the author wishes to thank Dr. Han, Dr. Foster, and Dr. Davidson for their many lm, ful suggestions, and particularly Dr. Starkey for his assistance, encourivement and enthusiasm during the project. 

Institute, Blacksburg, Virginia.

$1955-1956$

Maintenance Engineer, Esso Standard Oil Company, Baltimore, Maryland

$1956-1960$

Aeronautical Research Engineer, Aircraft Laboratory, Wright-Patterson AFB, Ohio

$1960-1963$

Chief, Mechanical Section, Air Force Flight Dynamics Laboratory, Wright-Patterson AFB, Ohio

1962

M.Sc., Miech. Engr., Ohio State University, Columbus, Ohio

$1963-1970$

Chief, Mechanical Brancin, Air Force Flight Dynaniics Laboratory, Wright-Patterson AFB, Ohio

\section{PUBLICATIONS}

"Design of Transparent Vision Areas for Orbital Glide Vehicles," presented at USAF/NASA Confercnce on Lifting Manned Hypervelocity and Re-entry Vehicles, Langley Research Center, Langley Field, Virginia, April 1 1-14, 1960.

"The Investigation of Techniques for Predicting the Decompression Rate of Closed Vessels Exhausting Through a Nozzle at Critical Velocity," M.S. thesis, Ohio State University, 1961.

"The Aerospace Challenge to Bearing Technology," presented at 1963 USAF Aerospace Fluids and Lubricants Conference, San Antonio, Texas, April 16-19, 1963. 
"An Analysis of Control Bearing Requirements for Lifting Re-entry Vehicles," Aeronautical Systems Division Technical Memo ASRMF-TM-63-7, June 1963.

"Design of High Temperature Aircraft Windows," presented at the Conference on Transparent Materials for Aerospace Enclosures, December 8-10, 1964.

"Results of Studies to Improve the Ground Flotation of Aircraft," presented at the SAE Aerospace Systems Conference, June 27-30, 1967.

"There Are Many Ways to Improve Ability of Aircraft to Land on Poorly Surfaced Fields," The SAE Journal, Vol. 76, No. 9, September 1968.

"Air Cushion Landing System for STOL Aircraft," V/STOL Technology and Planning Confercnce, September 23-25, 1969.

"Air Cushion Landing System Digest," AFFDL Technical Memorandum 69-10-FDFM, April 1969. 


\section{TABLE OF CONTENTS}

Acknowledgments

Page

Vita

iii

List of Illustrations

viii

List of Tables

xi

List of Symbols

xii

1. INTRODUCTION

1.1 Statement of the Problem

1.2 Background

1.3 The ACLS Concept

2. PERIPHERAL JET FLOW RELATIONSHIIPS 13

2.1 Method of Approach to Problem 13

2.2. Background 13

2.3 Development of Common Relationships 15

2.4 General Technique for Developing Flow Relationships 21

2.5 The Thin Jet Theory 31

2.6 The Exponential Theory 36

2.7 The Barratt Theory 39

2.8 Plenum Theory 46

3. COMPARISON OF FLOW THEORIES $\quad \cdot \quad 49$

3.1 Introduction 49

3.2 Recovery Pressure Ratio · $\quad 50$

3.3 Nozzle Thickness Parameter 52

3.4 Pressure Coefficient 52

3.5 Power Thickness Parameter 56

3.6 Power-Height Parameter 60

3.7 Augmentation Ratio 60

$3.8 \quad$ Summary of Results 63 
4. PREDICTION OF THE SHAPE OF A TWO DIMENSIONAL AIR CUSHION TRUNK

4.1 Approach

$\begin{array}{lll}4.2 & \text { Background } & 67\end{array}$

4.3 Development of Common Relationships 70

4.4 Free'Trunk Shape $\quad 75$

4.5 Loaded Trunk Shape 83

4.6 Trunk Cross Sectional Area 95

4.7. Analytical Results 97

5. ANALYSIS OF DISTRIBUTED JET FLOW

$\begin{array}{lll}5.1 & 120\end{array}$

5.2 Distributed Jet Momentum Theory 123

$\begin{array}{lll}5.3 & \text { Flow Restrictor Tineory } & 136\end{array}$

$\begin{array}{lll}5.4 & \text { Analytical Results } & 146\end{array}$

6. EXPERIMENTAL PROGRAM - STATIC MODEL 151

6.1 Experimental Apparatus - Static Tests 151

6.2 Experimental Proceciures - Static Test $\quad 153$

6.3 Summary of Results - Static Tests. 155

7. DYNAMIC ANALYSIS OF THE AIR CUSHION LANDING SYSTEIVI 175

$\begin{array}{lll}7.1 & 175\end{array}$

7.2 Simple Dynamic Model 180

7.3 Air Cushion Trunk Dynamic Analysis 189

7.4 Complete Air Cushion System Dynamic Analysis 205

8. EXPERIMENTAL PROGRAM - DYNAMIC MODEL 221

8.1 Experimental Apparatus - Dynamic Tests . 221

8.2 Determination of Discharage Coefficient $C_{x} \quad 226$

8.3 Determination of Jet Thrust and $C_{z} \quad 229$

8.4 Deternination of $\mathrm{A}_{3}$ and $\mathrm{C}_{y} \quad 229$

8.5 Determination of Trunk Volume 235

8.6 Fan Characteristics 240

8.7 Dynamic Model Test 242

8.8 Summary of Dynamic Test Results 247 
9. SUMMARY OF RESULTS 248

$\begin{array}{lll}9.1 & \text { Design Considerations } & 248\end{array}$

9.2 Aircraft Variables . 250

9.3 Jet System Variables 251

9.4 Trunik Variables 253

9.5 Power System Variables 256

9.6 Power Requirements for the ACLS 257

$\begin{array}{lll}9.7 & \text { Conclusions } & 259\end{array}$

$\begin{array}{lll}\text { Appendix I } & \text { Free Trunk Shape (Inelastic) } & 262\end{array}$

$\begin{array}{lll}\text { Appendix II } & 271\end{array}$

Appendix 1!l Elastic Free Trunk Shape 288

$\begin{array}{ll}\text { Appendix IV } & 291\end{array}$

Appendix $V \quad$ Determination of Flow Leakage 295

$\begin{array}{ll}\text { Appendix VI } & 297\end{array}$

$\begin{array}{ll}\text { References } & 299\end{array}$ 


\section{LIST OF ILLUUSTRATIONS}

Figure

Page

1-1 Air Cushion Suspension System 3

1-2 Historical GETOL Designs

1-3 Artist's Concept of the Air Cushion Landing System

$\begin{array}{lll}1.4 & \text { Braking System for ACLS } & 8\end{array}$

1-5 ACLS Footprint Pressure Distribution 8

1.6 Historical Air Cuslion Vehicles $\quad 10$

1.7 Comparison of Air Cushion Designs 12

2-1 Air Cushion Model Configuration $\quad 17$

2-2. Model for General Theory 23

2-3 Nodel ior Thin Jet and Exponential Theory 32

2-4 Model for Barritt Theory 40

3-1 Nozzle Thickness Parameter versus $\mathrm{P}_{\mathrm{c}} / \mathrm{P}_{\mathbf{j}} \quad 51$

3-2 Flow Coefficient vorsus $P_{c} / P_{j}$

3-3 Power-Thickness Parameter versus $P_{c} / P_{j}$

3-4(a) Power-Height versus $P_{c} / P_{j}$, Simple Jet Theory 57

3-4(b) Power-Height Porameter versus $P_{c} / P_{j}$, Exponential Theory 58

3-4(c) Power-Height Parameter versus $P_{c} / P_{j}$, Barratt Theory 59

3-5 Augmentation versus Jet Height to Cushion Diameter Ratio 62

$\begin{array}{lll}\text { 4-1 Free Trunk Shape } & 68\end{array}$

4-2 Loaded Trunk Shape $\quad 69$

4-3 Fres Body Diagram of Trunk Loading $\quad 74$

4-4 Physical Interpretation of Positive and Negative Square Root 80

4.5 Lllustration of Minimum Trunk Length 90

4-6 Geometry for Calculating the Upper Bound for $R_{1}$. 92

4-7 Physically Impossible Solution $\quad 93$

4-8 Air Cushion Model $\quad 99$

4-9 Side Trunk Shape 100

4-10. End Trunk Shape 101

4-11 $Y_{\mathrm{o}}$ versus $P_{c} / P_{j}$, Side and End Truink 103

4-12 $X_{o}$ versus $P_{c} / P_{j}$, Side and End Trunk. 104

4-13 $\quad A_{j}$ versus $P_{c} / P_{j}$, Side and End Trunk . 105

4-14 Elastic Curve for Trunk Material 107 
4-15 Trunk Length versus $P_{c} / P_{j}$, Elastic Side Trunk

4-16 $Y_{o}$ versus $P_{c} / P_{j}$, Elastic Side Trunk

4-17 $\quad Y_{o}$ versus $P_{c} / P_{j}$, Elastic End Trunk

4-18 $\quad A_{j}$ versus $P_{c} / P_{j}$, Elastic Side Trunk

4-20 $Y_{o}$ versus $P_{C} / P_{j}$, Comparison of Results

4-21 $l_{3}$ versus $Y_{0} / Y_{\text {co, }}$ Side Trunk

4-22 $\quad l_{3}$ versus $Y_{0} / Y_{\infty}$, End Trunk

4-23 $\quad A_{j}$ versus $Y_{o} / Y_{\infty}$ Side Trunk

4-24 $\quad A_{j}$ versus $Y_{o} / Y_{\text {Dor }}$ End Trunk

5-1 Distributed Jet Geometry

5-2 Trunk Geometry for Distributed Jet

5.3 Three Cases for Jet Locations 133

5-4 Location of Jets Relative to Low Point 139

5-5 Typical Jet Spacing $\quad 140$

5-6 Analytical Predictions of $d / t$ versus $P_{c} / P_{j}$-Model Side Trunk 148

5.7. Analytical Predictions of $C_{Q}$ versus $P_{c} / P_{j}$-Model Sicle Trunk 149

6.1 Static (2D) Test Rig $\quad 152$

6-2 $\quad \ell_{1}$ versus $P_{c} / P_{j}$ Results $\quad$. 156

6.3 $X_{o}$ versus $P_{c} / P_{j}$ Results $\quad 157$

6.4 $\quad Y_{0}$ versus $P_{c} / P_{j}$ Results 158

6.5 Trunk Shape Results 159

6.6 $\quad l_{3}$ versus $Y_{o} / Y_{\infty}$ Results, $P_{c} / P_{j}=0 \quad 161$

6.7 $l_{3}$ versus $Y_{o} / Y_{\infty}$ Results, $P_{c} / P_{j}=0.41 \quad \cdot 162$

6.8 Cushion Exhaust Pressure Distribution, $P_{c} / P_{j}=0.28 \quad 168$

6-9 Cushion Exhaust Pressure Distribution, $P_{c} / P_{j}=0.52 \quad 169$

6-10 Cushion Exhaust Pressure Distribution, $\mathrm{P}_{\mathrm{C}} / \mathrm{P}_{\mathrm{j}}=0.7 \quad 170$

6.11 $C_{Q}$ versus $P_{c} / P_{j}$, Results 173

6-12 $d / t$ versus $P_{c} / P_{j}$ Results $\quad 174$

7-1 Simple Model for Dynamic Analysis $\quad$. 179

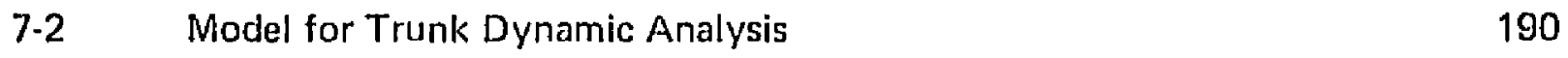

7.3 Model for Pressure Distribution Across the Footprint 199

7.4 Load-Deflection Characteristics of the Cushion Exhaust Gap 200

7-5 Free Body Diagram for Trunk Footprint 201 
7-6 Model for Air Cushion System Dynamic Analysis 204

$\begin{array}{lll}7.7 & X_{0} \text { versus } Y \text { for Model Trunk } & 208\end{array}$

7.8 $\quad M_{2}$ versus $Y_{0}$ far Model Trunk $\quad 213$

7-9 Centroidal Radius versus Trunk Height for Model Trunk 216

8-1 Dyuamic Model and Test Platform 222

8.2 Hydraułic Mótor Characteristics 224

8.3 Fan Chanacteristics $\quad 225$

8.4 Trunk Discharge Cofficient versus $\mathrm{P}_{\mathrm{A}} / \mathrm{P}_{\mathrm{j}} \quad 227$

8-5 Thrust 'iersus Trunk Pressure $\quad 228$

8.6 Trunk Pressure and Jet Height Variation with Vehicle Height 230

8.7 Footprint Alea versus Vehicle Height 231

8-8 Cushion Discharge Cocticient versus Vehicle Height 234

8-9 Trunk Volume Ratio versus Vehicle Height Ratio 239

8-10 Assumed Fan Characteristics $\quad 241$

8-11 Trunk Pressure During Drop Test 244

8-12 Accerleration During Drop Test 245

8-13 Displacement During Drop Test 246

9-1 Power Height Parameter for Two Trunk Designs 254

9-2 Load Deflection Characteristics • 258

9-3 Fuselage Area versiss A/C Gross Weight 258

9.4 Fuselage Perimeicr versus A/C Gross Weight 258

9.5 ACLS Power versus A/C Weight 258 


\section{LIST OF TABLES}

Table

Page

3-1 Expressions for $\mathrm{pc} / \mathrm{pj}$ and $\mathrm{C}_{\mathrm{Q}}$ for Momentum Flow Theories 66

4.1 Trunk Model Dimensions 98

5.1 Values of Trunk Design Variables $\quad 124$

6.1 Pressure Ratio $\left(\mathrm{p}_{\mathrm{C}} / \mathrm{p}_{\mathrm{j}}\right)$ vs Vehicle Height $(H)$ and Trunk Pressure $\left(\mathrm{p}_{\mathrm{j}}\right) \quad 164$

6-11 Flow Theory Coefficient $\left(\mathrm{C}_{\mathrm{Q}}\right)$ vs Vehicle Height $(H)$ and Trunk Pressure: $\left(\mathrm{P}_{\mathrm{j}}\right) \quad 165$

6-III Jet Height- Thickness Ratio (d/t) vs Vehicle Height $(H)$ and Trunk Pressure $\left(\mathrm{p}_{\mathrm{j}}\right)$

G.IV Calculated Datu vs Vehiche Height (H) 167

8.1 Dynamic Model Trunk Design Variables 223 


\section{LIST OF SYMBOLS}

Latin letters

A piston area, $\mathrm{ft}^{2}$

$A_{c} \quad$ cushion area, $\mathrm{rt}^{2}$

$A_{g} \quad$ cushion area under trunk, $\mathrm{ft}^{2}$

$A_{h} \quad$ cushion arca under jircraft hard structure, $\mathrm{ft}^{2}$

$A_{1}$ jet augmentation ratio

$A_{j} \quad$ cross-sectional area of the trunk, $\mathrm{ft}^{2}$

$A_{3}$ trunk footprint area, $\mathrm{ft}^{2}$

a $\quad x$ coordinate of upper trunk attachment point, $\mathrm{ft}$.

$\bar{a} \quad$ horizontal distance between trunk attachment points, $\mathrm{ft}$

$a_{D}$ total area of exhaust nozzle for fan calibration test, $\mathrm{ft}^{2}$

$a_{j} \quad$ total area of all orifices in the trunk, $\mathrm{ft}^{2}$

$a_{n}$ total arca of all orifices in the $n$th segment of the trunk, $\mathrm{ft}^{2}$

$a_{r} \quad$ effective flow area for fan backflow, $\mathrm{rt}^{2}$

$a_{3}$ ' effective flow area for the $\ell_{3}$ segment of the trunk, $\mathrm{ft}^{2}$

$\mathrm{b} \quad \mathrm{y}$ coordinate of upper trunk attachment point, $\mathrm{ft}$

$C_{D} \quad$ coefficient of discharge for plenum chamber

$C_{\text {hd }} \quad$ power - jet height parameter

$\mathrm{C}_{\mathrm{ht}} \quad$ power-thickness parameter

$\mathrm{C}_{\mathrm{p}} \quad$ specific heat at constant pressure, $\mathrm{Btu} / \mathrm{lb} \mathrm{b}^{\circ} \mathrm{F}$

$\mathrm{C}_{\mathrm{Q}} \quad$ flow coefficient for pressure distribution across the jets

$\left(\mathrm{C}_{\mathrm{Q}}\right)_{\mathrm{n}} \quad$ flow coefficient for pressure distribution across the nth row of jets

$\mathrm{C}_{\mathrm{T}} \quad$ percent reduction in flow area of cushion exhaust caused by trunk jets

$\mathrm{C}_{\mathrm{v}} \quad$ coefficient of discharge for the trunk

$\mathrm{C}_{\mathrm{X}} \quad$ coefficient of discharge for the trunk nth row of orifices in the trunk 


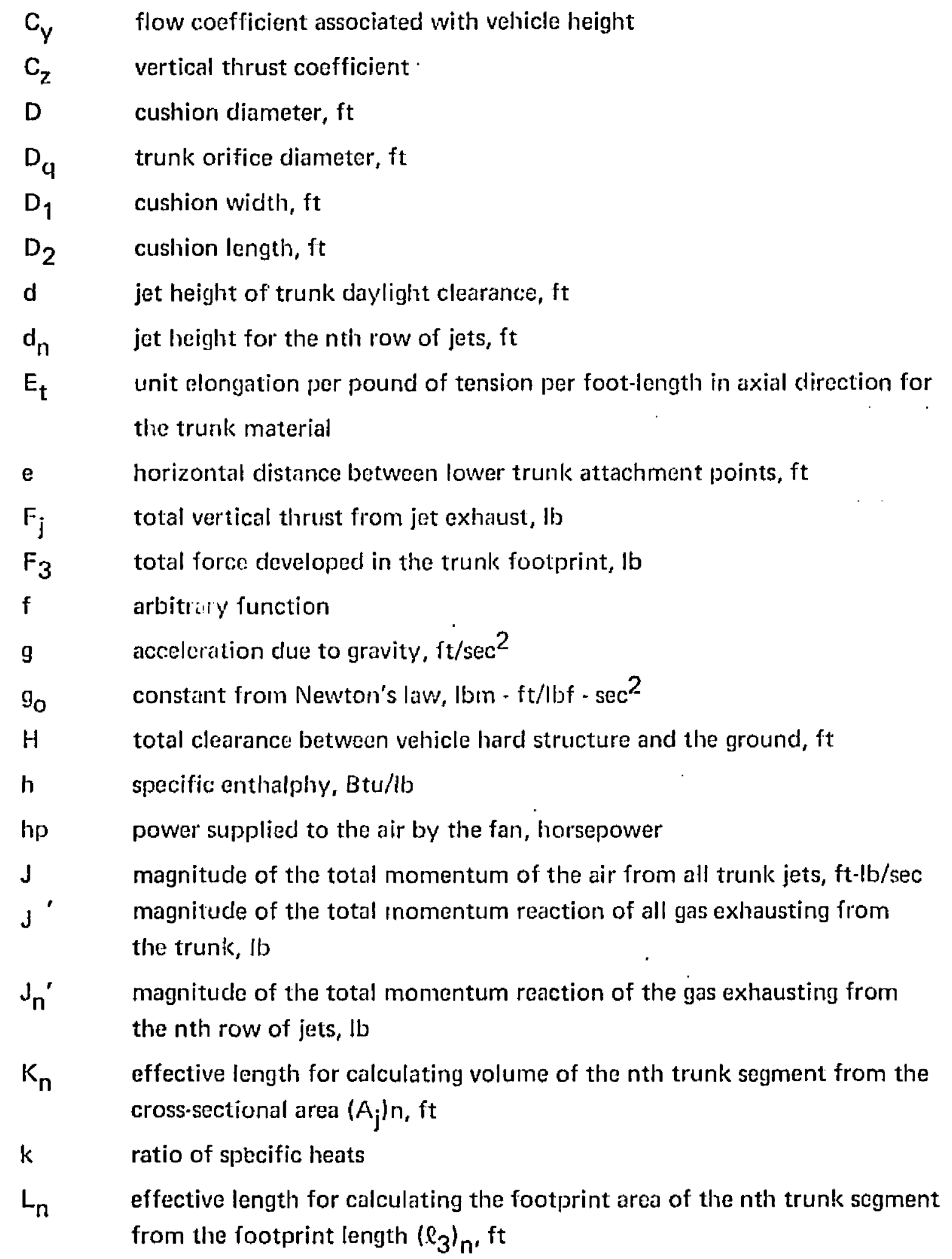




\begin{tabular}{|c|c|}
\hline$L_{s}$ & length of trunk side segment, $\mathrm{ft}$ \\
\hline$\ell$ & cross-sectional length of trunk, $\mathrm{ft}$ \\
\hline $\bar{\ell}$ & trial value of the cross-sectional length of trunk, $\mathrm{ft}$ \\
\hline$\ell_{n}$ & length of trunk segment $\mathrm{n}, \mathrm{ft}$ \\
\hline$\ell_{0}$ & design lengt/i of trunk cross-section, $\mathrm{ft}$ \\
\hline M & total number of rows of orifices \\
\hline$M_{n}$ & number of rows of orifices \\
\hline $\mathbf{m}$ & $\begin{array}{l}\text { mass per uniz width of an infinitesimal elemer of gus in jet :- :e figute } \\
2-2) \text {, slugs/ft }\end{array}$ \\
\hline$\dot{\mathrm{m}}$ & mass flow rate, slugs/sec \\
\hline$N$ & number of jet orifices per row \\
\hline$n^{\prime}$ & $\begin{array}{l}\text { effective number of rows or orifices which contribute to cushion exhaust } \\
\text { nozzle area reduction }\end{array}$ \\
\hline$P(p)$ & pressure, psfa $(p, f:)$ \\
\hline $\mathrm{P}_{\mathrm{a}}$ & atmospheric pressure, !sfa \\
\hline$P_{c}\left(p_{c}\right)$ & cushion presi. $r \ldots$, psfa (psfg) \\
\hline$P_{j}\left(p_{j}\right)$ & trunk pressure, pefa $(t) i g)$ \\
\hline$P_{\mathfrak{n}}$ & static pressure in cushion exhaust nozzle at nth row of trunk orifices, psf \\
\hline $\mathrm{P}_{\mathrm{c}} / \mathrm{P}_{\mathrm{j}}$ & cusinion to trunk pressure ratio (both pressures in psfg) \\
\hline$p_{c} / p_{j}$ & cushion to trunk pressure ratio (both pressures in psfg) \\
\hline $\mathbf{Q}$ & flow rate, $\mathrm{ft}^{3} / \mathrm{sec}$ \\
\hline $\mathrm{O}_{\mathrm{c}}$ & total flow from the cushion, $\mathrm{ft}^{3} / \mathrm{sec}$ \\
\hline $\mathrm{Q}_{\mathbf{i}}$ & total flow from the fan, $\mathrm{ft}^{3} / \mathrm{sec}$ \\
\hline $\mathrm{o}_{\mathrm{j}}$ & total flow from the trunk, $\mathrm{ft}^{3} / \mathrm{sec}$ \\
\hline $\mathrm{a}_{\mathrm{L}}$ & leakage flow, $\mathrm{ft}^{3} / \mathrm{sec}$ \\
\hline$a_{n}$ & total flow from the orifices in the $\left(l_{n}\right)^{\text {th }}$ trunk segment, $\mathrm{ft}^{3} / \mathrm{sec}$ \\
\hline$Q_{p}$ & total flow from the plenum chamber, $\mathrm{ft}^{3} / \mathrm{sec}$ \\
\hline$Q_{r}$ & total fan flow at stall pressure, $\mathrm{ft}^{3} / \mathrm{sec}$ \\
\hline$\left(O_{t}\right)_{n}$ & total flow from the jets in row $n$ through row $\mathrm{m}, \mathrm{ft}^{3} / \mathrm{sec}$ \\
\hline
\end{tabular}




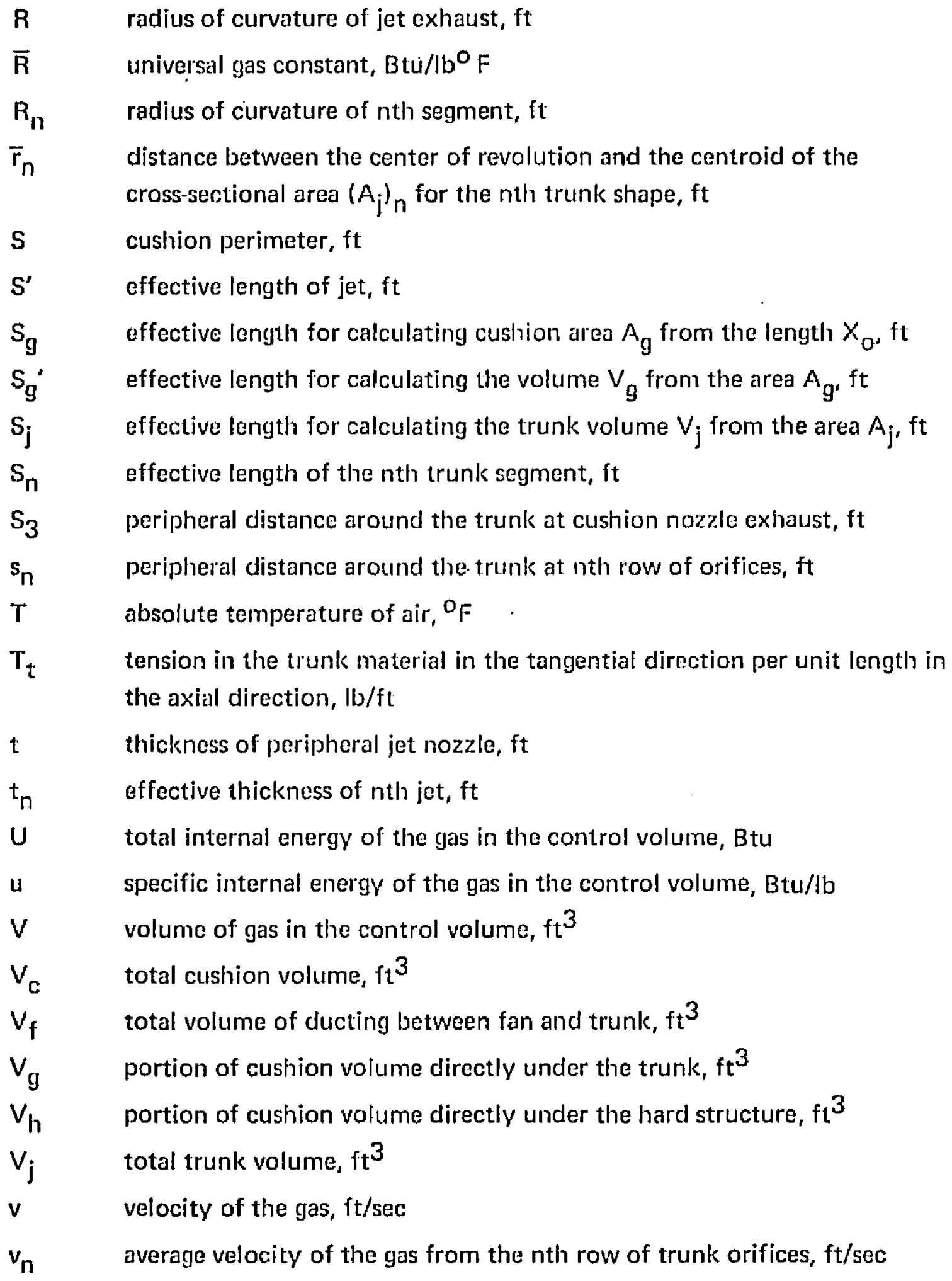




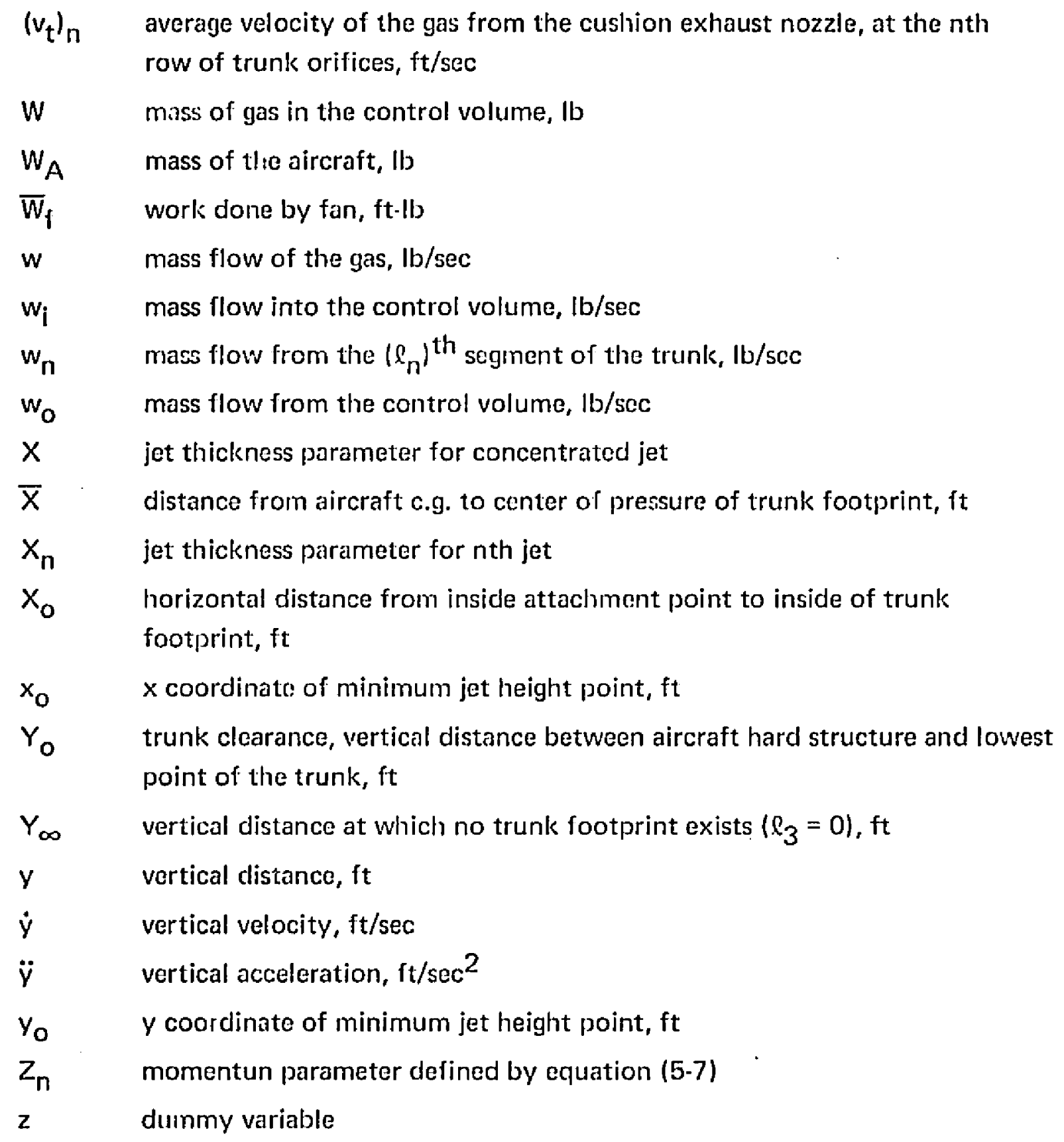

Greek letters

$\alpha_{n} \quad$ angle of revolution for trunk cross-section to form trunk volume segment $n$, radians

$\beta_{n} \quad$ angular position of $n$th row of orifices relative to the vertical, radians 


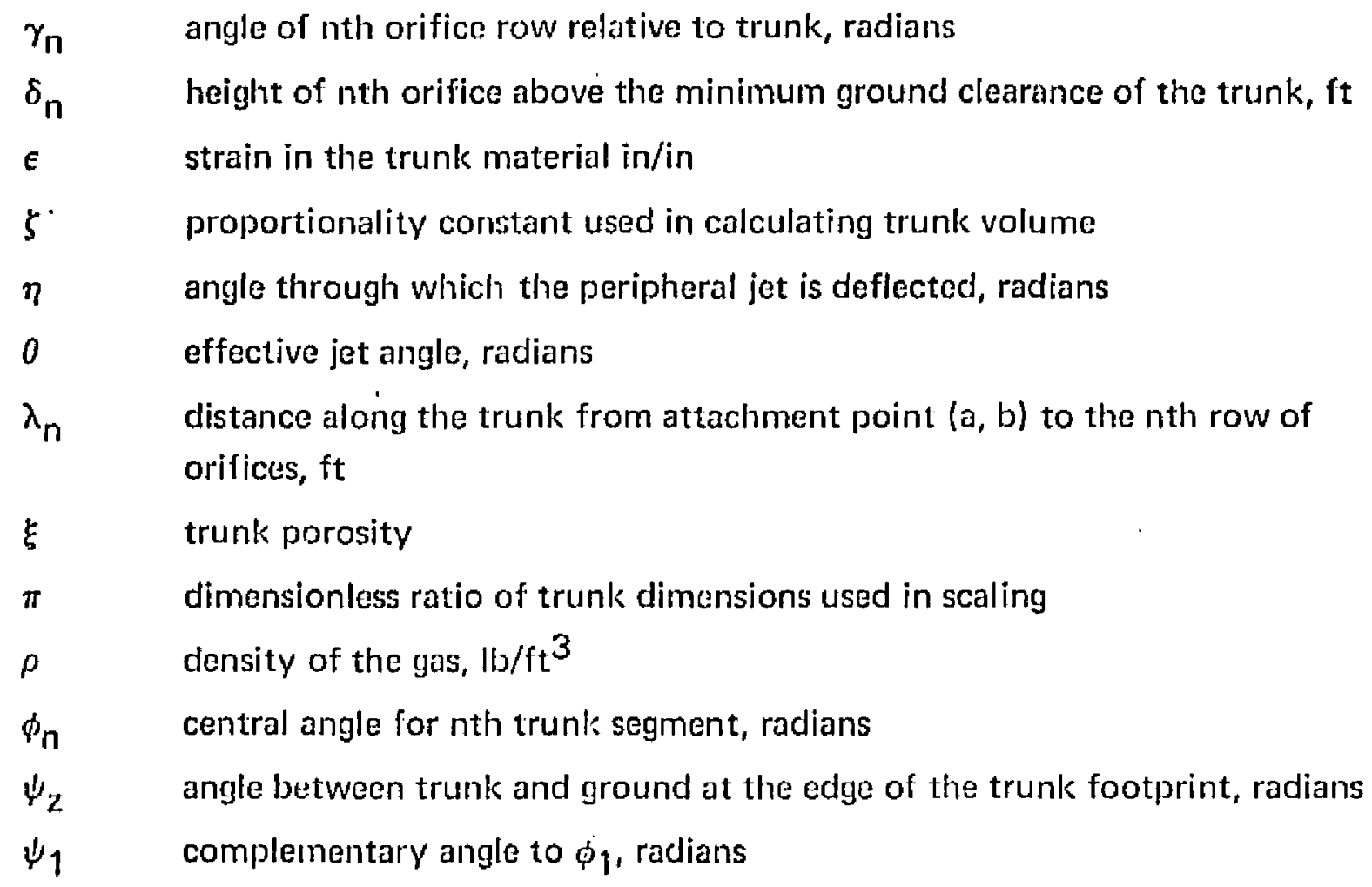

Subscripts

$\begin{array}{ll}\text { A } & \text { aircraft } \\ \text { a } & \text { atmosphere } \\ \text { c } & \text { cushion } \\ \text { e } & \text { end section of trunk volume } \\ \text { f } & \text { fan } \\ \text { g } & \text { cushion volume under trunk } \\ \text { h } & \text { cushion volume under hard structure } \\ \text { i } & \text { flow into the control volume } \\ \text { j } & \text { trunk } \\ k & \text { corner section of trunk volume } \\ \text { l } & \text { first row of jet orifices inside the cushion } \\ \text { m } & \text { last row of jet orifices inside the cushion } \\ n & \text { arbitrary }\end{array}$




$\begin{array}{ll}\text { o } & \text { flow out of the control volume } \\ \mathbf{q} & \text { plenum chamber } \\ \mathbf{r} & \text { orifices } \\ \mathbf{s} & \text { stall conclition of the fan } \\ \mathbf{t} & \text { side section of trunk volume } \\ 0 & \text { total value of all parts } \\ 1 & \text { atmosphere } \\ 2 & \text { trunk seginent inscribed by angle } \phi_{1} \\ 3 & \text { trunk segment inscribed by angle } \phi_{2} \\ 4 & \text { trunk segment flat tened against the ground } \\ \infty & \text { distance at which trunk support is negligible }\left(l_{3}=0\right)\end{array}$




\section{INTRODUCTION}

\subsection{Statement of the Problem}

The purpose of this work is to develop design techniques which can predict analytically the power requirements and dynamic response of a unique air suspension system which can be used to replace the landing gear on aircraft. The particular system analyzed will be referred to as the Air Cushion Landing System and abbreviated ACLS. The ACLS was developed jointly by Bell Aerosystems and the Air Force Flight Dynamics Laboratory. It utilizes a flexible skirt or "trunk" and a distributed poripheral jet as described in Section 1.3. The development program for the ACLS is documented by References $1,2,3,4,5$, and 6 . The referenced program was largely experimental. This study is intended to present analytical techniques which will be useful in extrapolating the reported experimental results and in designing larger and more efficient air suspension systems for aircraft.

The power requirements for an air suspension system may be stated in terms of pressure versus flow characteristics for the fan which supplies the air for the system. In the following chapters, relationship: are developed which relate the pressure and flow to the resulting ground clearance and overpressure beneath the aircraft. For the purposes of this work, the effect of forward velocity is neglected.

The dynamic response of interest in this work is the response of the air cushion trunk to landing impact. It is desired to predict the forces and motions which result from a residual vertical velocity of the aircraft at touchdown. Of particular interest are the maximum acceleration and the maximum trunk deflection for a given aircraft weight and sink rate. For the purpose of this work, only vertical forces and motions are considered. 
Aerodynamic forces resulting from the aircraft surfaces are neglected as are all moments and angular motions.

Static analyses of the trunk shape and flow churacteristics are prerequicites to analytical treatment of both power requirements and the dynamic response of the stem. Consequently, these analyses are developed and experimentally verified prior to presenting the dynamic and power system analysis.

The most widely accepted flow theories for predicting the cushion pressure in air cushion vehicles are summarized in Chapter 2. Nondimensional flow parameters are developed in Chapter 3. The prediction of the trunk shape and cross-sectional area is developed in Chapter 4. Flow theories for the combined trunk-jet system are presented in Chapter 5. Experimental results to verify the trunk shape and flow theories are presented in Chapter 6. An analysis of the dynamic response of the trunk system is derived in Chapter 7. Experimental verification of the dynamic system is presented in Chapter 8.

A summary of the design considerations, the dynamic response and the power requirements is included in Chaptor 9.

\subsection{Background}

An air suspension system supports a vehicle on a cushion of air trapped between the vehicle underside and the ground. The vehicle weight is uniformly distributed by the air cushion over a large area. Extremely low ground pressure results. Consequently, such a system offers the potential for operating on extremely soft ground and even water.

The two most common air suspension systems are known as the plenum chamber and the peripheral jet. These systems are illustrated in Figures 1-1(a) and 1-1(b), respectively. Both systems rely on "ground effects" or an overpressure caused by the presence of the ground for support. In both systems, input power is required to maintain the air cushion. The major difference between the two systems lies in the mechanism by which the overpressure is maintained. The plenum chamber utilizes a flow restriction, while the peripheral jet maintains the overpressure by a momentum "seal". 
3

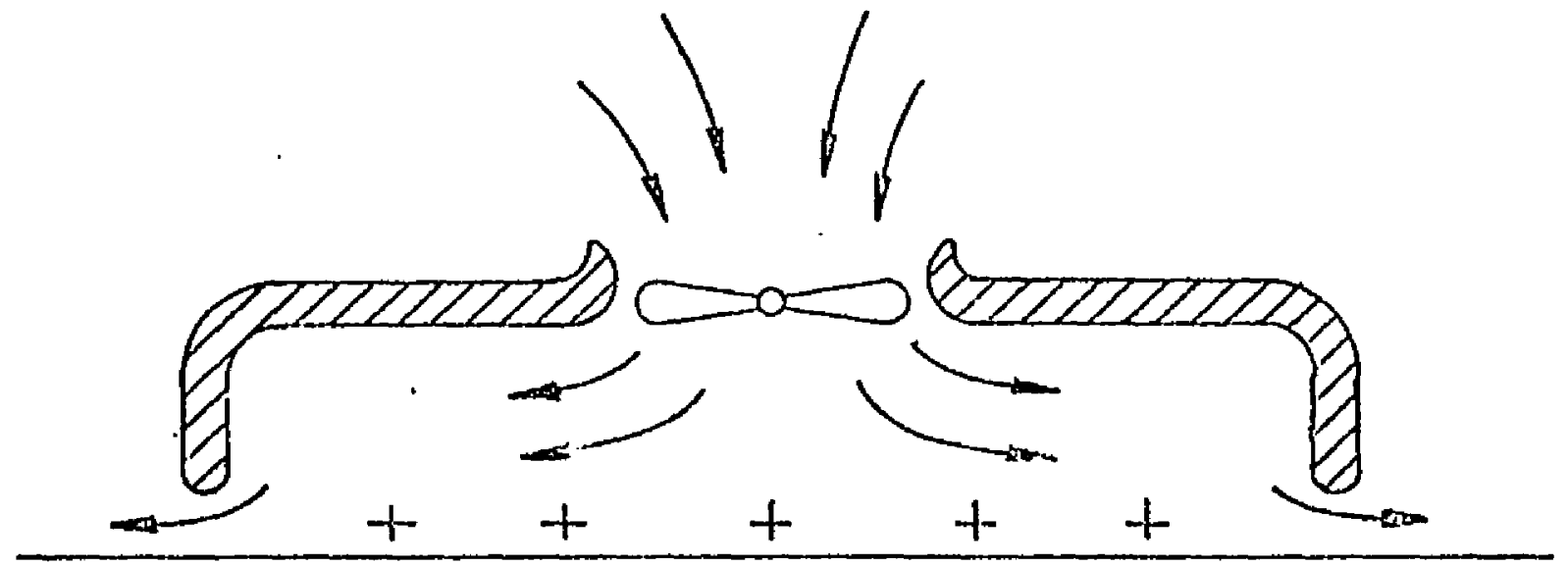

(a) Plenum chamber

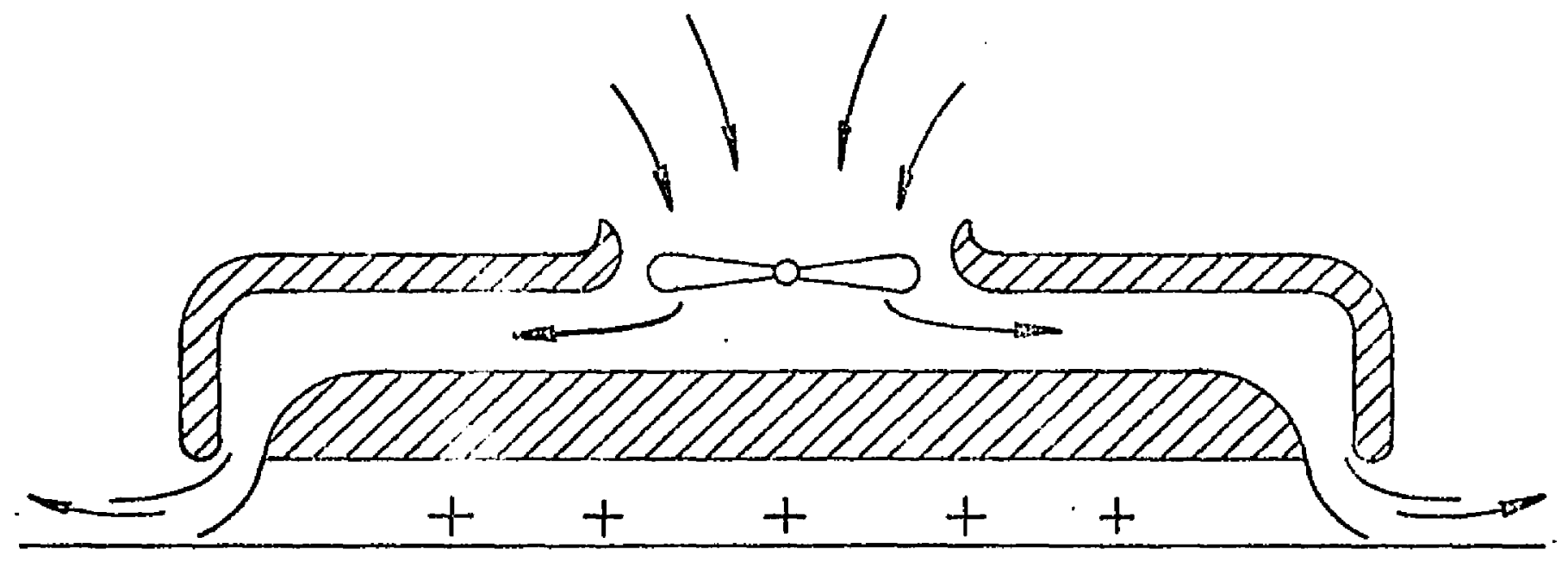

(b) PERIPHERAL JET

AIR CUSHION SUSPENSION SYSTEMS

FIGURE 1-1 
In the case of the plenum chamber, air is pumped into the cavity under the vehicle and leaks out through a narrow yap between the periphery of the vehicle and the ground. An overpressure is maintained in the cavity as a consequence of equilibrium between the pressure differential across the gap and the combined acceleration and frictional forces which limit the flow of air through the gap. The result is a flow restriction of the exhaust plane.

In the cise of a peripheral jet, air is vented in a jot at the periphory to form an air curtain seal. The scaling effect of the jet is a consequence of the equilibrium between the pressure differential across the jet and the centrifugal forces in the curved jet airflow. Pressure in the cushion is maintained by this air curtain seal. In a "pure" peripheral jet air suspension system, all air is introduced at the periphery. In theory, air neither enters nor leaves the cavity when the system is at equilibrium.

The concept of using an air cushion (or ground elfects) to support an aircraft during take-off and landing is not new. Machines which utilize this principle are called Ground Effects Take-off and Landing aircraft and are abbreviated GETOL aircraft. Studies of GETOL concepts have been conducted by AVRO Canada, ONERA (France), UTIAS (Canada), DORNIER (Gelmany) and VERTOL and CONVAIR, and Bell Aerosystems in the United States of America. $(7,8)$ *

Figure 1-2(a) shows the AVROCAR, a peripheral jet concept which was studied by AVRO between 1954 and 1962 . $^{(9,10,11,12,13)}$ Research was discontinued because of excessive power consumption (attributed to high duct losses) and instability when out of ground effect.

Figure 1-2(b) shows a GETOL aircraft design proposed by VERTOL. The VERTOL studies indicated that their design is competitive with conventional aircraft in weight and performance. $(14,15,16,17,18)$ However, the static and dynamic stability and control of the craft present major problems.

\footnotetext{
* Numbers in parentheses refer to references.
} 


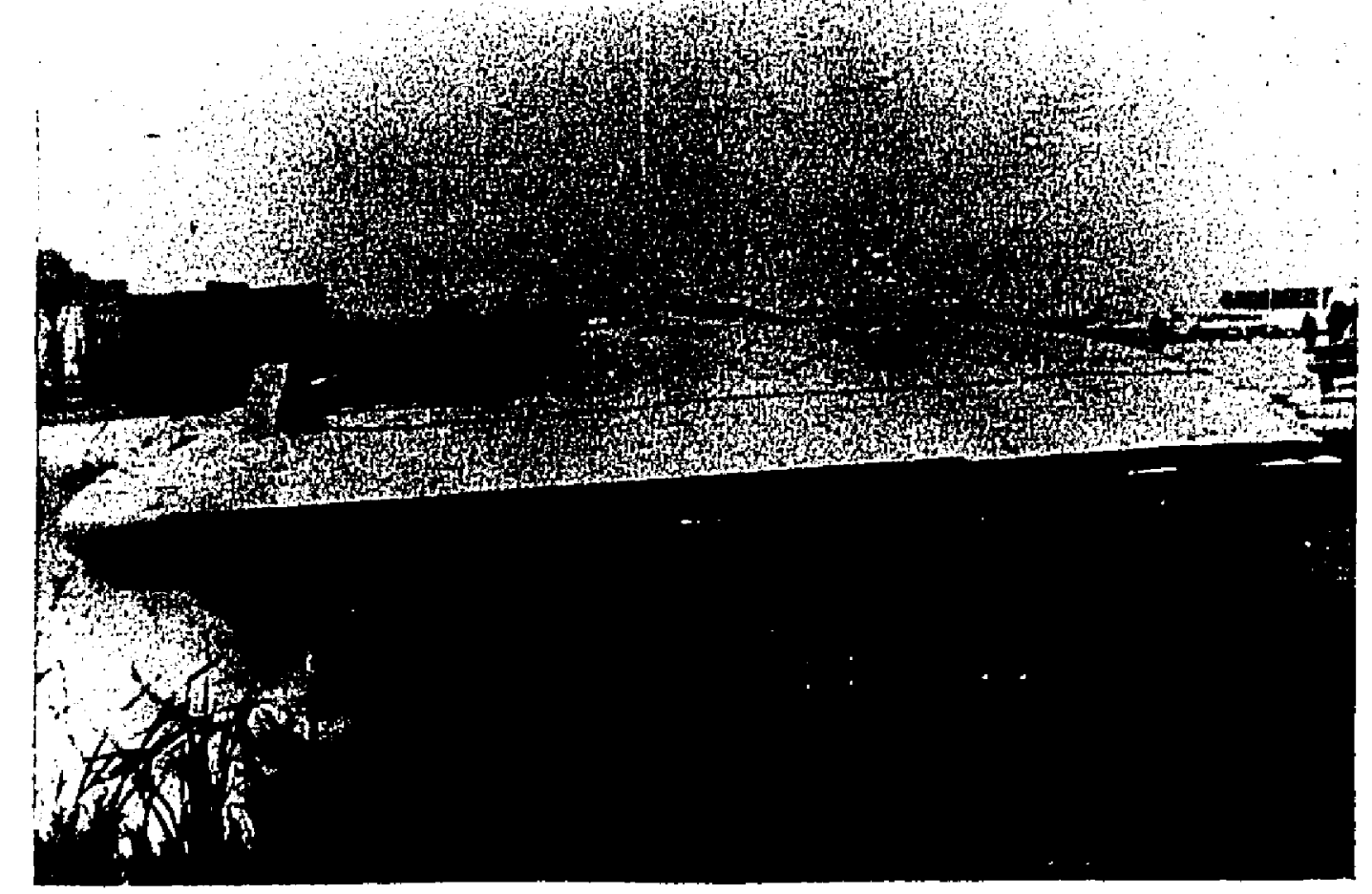

(a) THE AVROCAR

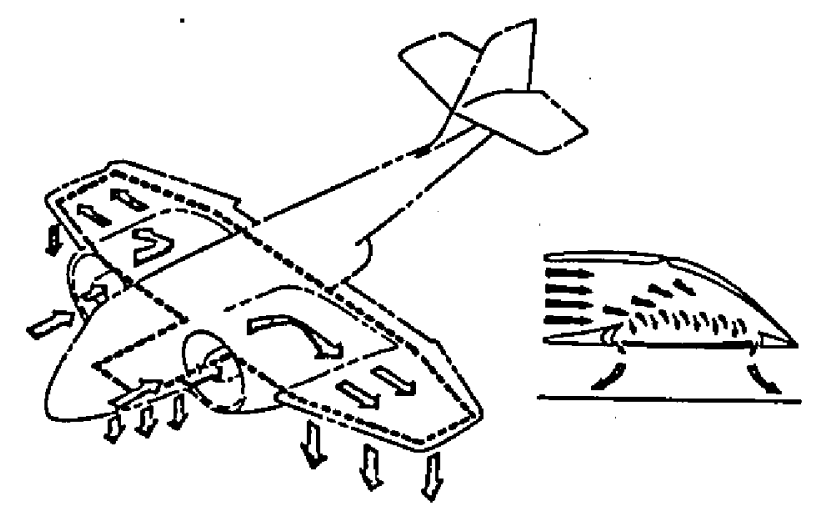

(b) VERTOL PROPOSED GETOL

historical getol designs

FIGURE 1-2 
CONVAIR studied a GETOL aircraft with a thick rectangular wing equipped with a peripheral nozzle. $(19,20)$ The major difficulties anticipated were stability and excessive energy losses.

ONERA, ${ }^{(21,22)}$ UTIAS $(23,24,25,26)$ and DORNIER ${ }^{(27)}$ have studied wings of various shapes equipped with peripheral nozzles. Each of the studies mentioned above employed a jet height (ground clearance) measurable in feet. Several deficiencies are associated with such large ground clearances. These deficiencies include poor stiffness, poor vertical cnergy absorptive properties and large power requirements.

The concept developed jointly by Bell and the Air Force Flight Dynamics Laboratory is unique. $(1,2,3,4)$ It utilizes a jet height of less than one inch, thus reducing the power requirements to an acceptable level. The use of flexible skirts around the periphery of the air cushion greatly increases the stiffness and energy absorptive properties of the system.

\subsection{The ACLS Concept}

The Air Cushion Landing System completely eliminates the conventional aircraft landing gear and replaces it with a cushion of air maintained beneath the fuselage during take-off and landing. An artist's concept of the system is shown in Figure 1-3. The elongated doughnut shown on the bottom of the fuselage is called a trunk. The trunk forms the flexible ducting required to provide a continuous curtain of air around the periphery of the fuselage,

Air is fed into the trunk from a compressor located in the nose wheel well. The air is ducted by the trunk to the fuselage periphery and exhausted through jets in the trunk to form a jet curtain. This jet curtain seals a pressure of one to two psi under the aircraft fuselage when the ground is approached. The trunks are made of rubber and nylon. When inflated, they stretch approximately $300 \%$ to assume the shape shown in Figure 1-3. When not pressurized, they shrink and hug the fuselage like a de-icing boot.

A braking system is shown in Figure 1-4. Braking is accomplished by pressing a brake material against the ground. The brake material may be replaced without replacing the 


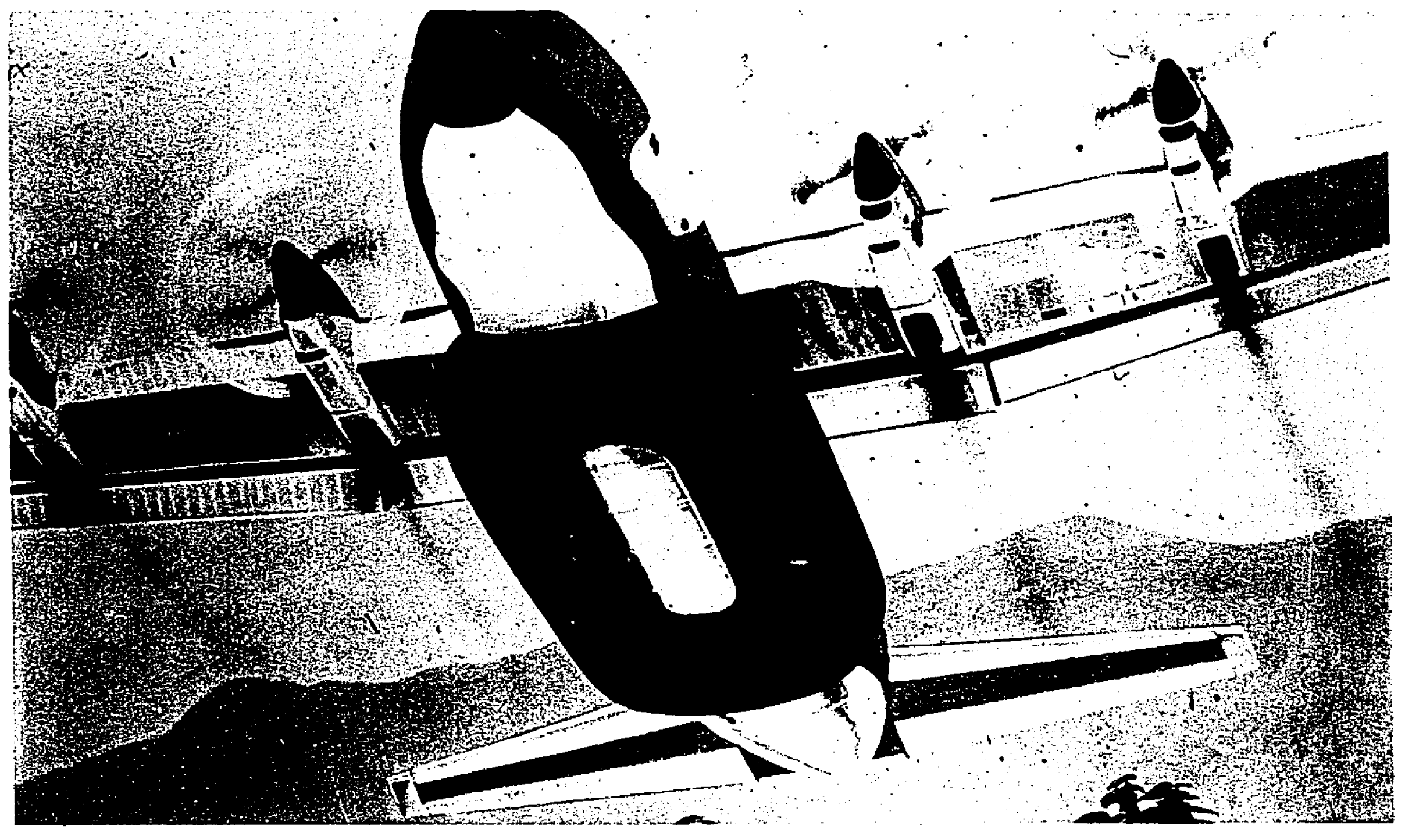

ARTIST'S CONCEPT OF THE AIR CUSHION

LANDING SYSTEM

FIGURE 1-3 


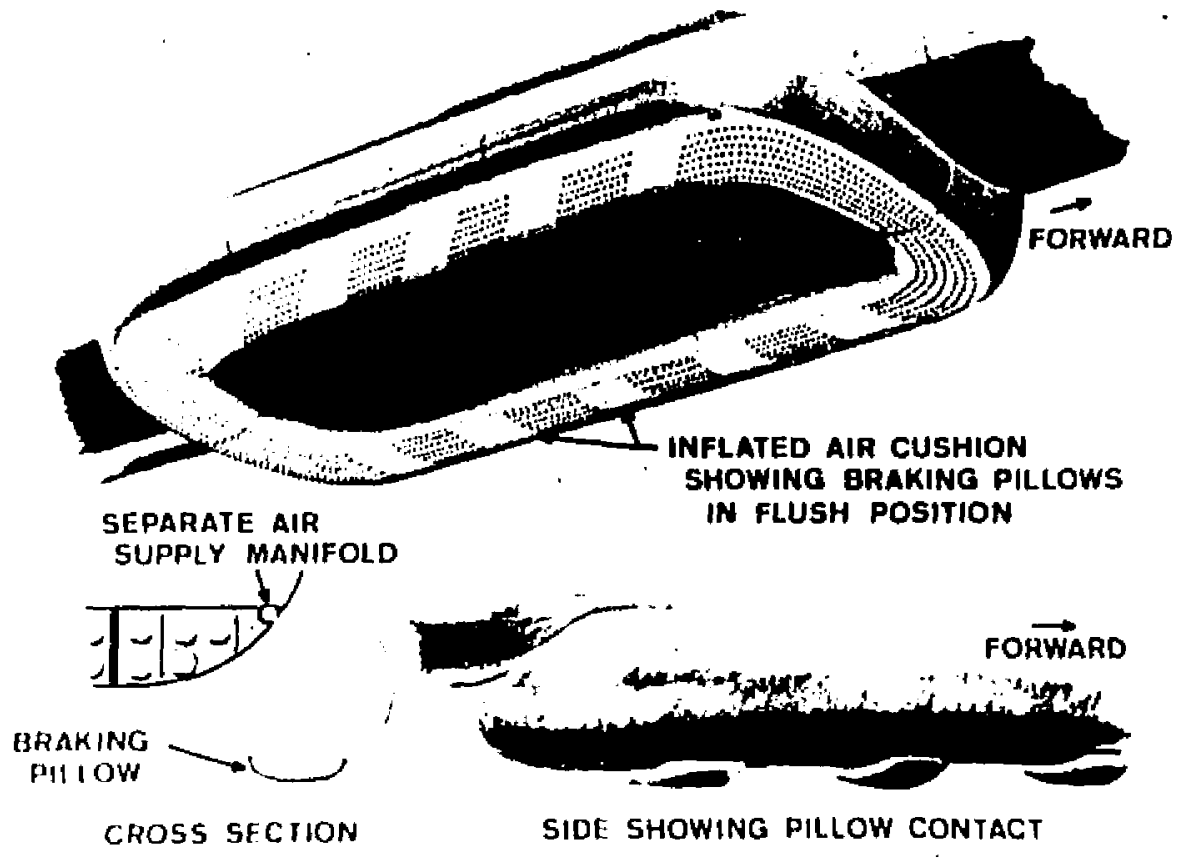

\section{BRAKING SYSTEM FOR ACLS \\ FIGURE I-4}

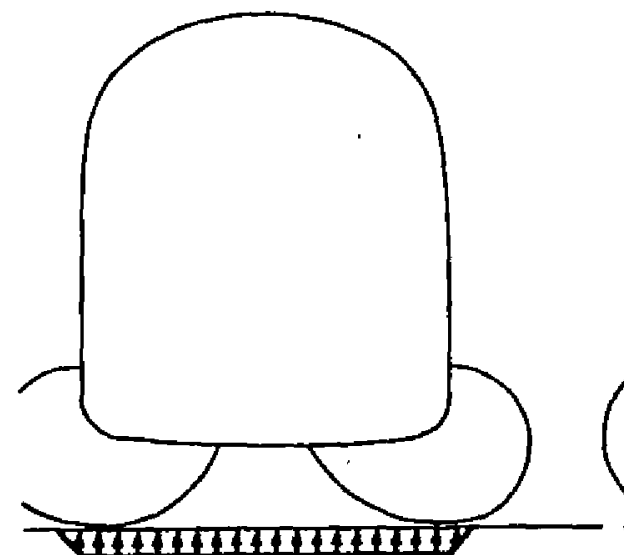

NORWKL FOOTPRIKT PRESSURE DISTRIBUTION

(a)

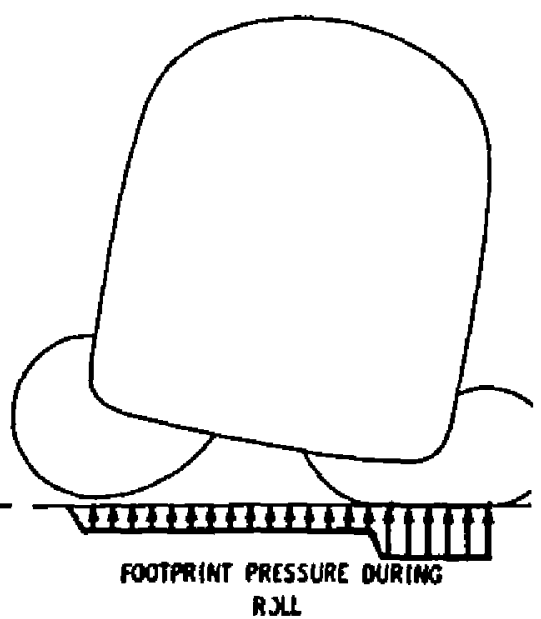

(b)

\section{ACLS FOOTPRINT PRESSURE DISTRIBUTION \\ FIGURE I- 5}


rest of the system - just as conventional brakes may be relined without replacing the landing gear. Brakes are actuated by applying pneumatic pressure to the pillow sections shown on the bottom of the trunk. Steering is accomplished by differential braking as in a caterpillar tractor.

The mechanism by which roll angles are reacted is shown in Figure 1-5. The figure on the leit shows the approximate footprint pressure of the ACLS under equilibrium conditions. The aircraft is totally supported by the cushion of air maintained under the fuselage. Under a large roll angle, the footprint pressure changes. The change is shown in the right figure. In addition to the cushion of air, the trunk is supporting the aircraft. The pressure in the trunk is roughly twice the pressure in the cushion. The trunk pressure, acting over the area shown in Figure 1-5, clevelops a large restoring moment whenever the bag is flattened against the ground. Negligible scrubbing of the bag against the ground occurs due to the large flow of air between the bag and ground. Very low friction results. The phenomena by which pitch stiffness is obtained is identical to that by which roll stiffness is obtained.

This Air Cushion Landing System is an extension of the technology developed for air cushion vehicles. Figure 1.6(a) shows one such vehicle used by the U.S. Army in Vietnam. ${ }^{(28)}$ This vehicle weights about eight tons. A larger vehicle built for the Navy by Bell Aerosystems is shown in Figure 1-6(b). This vehicle weighs about 30 tons approximately equal to the $\mathrm{C}-119$ and $\mathrm{C}-123 .{ }^{(7,28)}$ The British operate a velicle which weighs 163 tons, or nearly twice the weight of the $\mathrm{C}-130 .{ }^{17,28)}$ This vehicle, shown in Figure 1-6(c), provides commercial ferry service across the English Channel.

An extensive amount of work has been published concerning the performance of Air Cushion Vehicles. ${ }^{(8)}$ Much of this work can, and has been applied to predicting the static performance of the ACLS. However, the design of the trunks and the peripheral nozzles on the ACLS are considerably different from the design of the same items on Air 


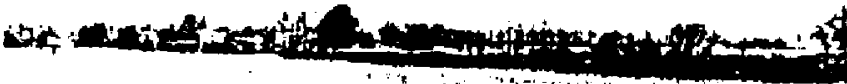

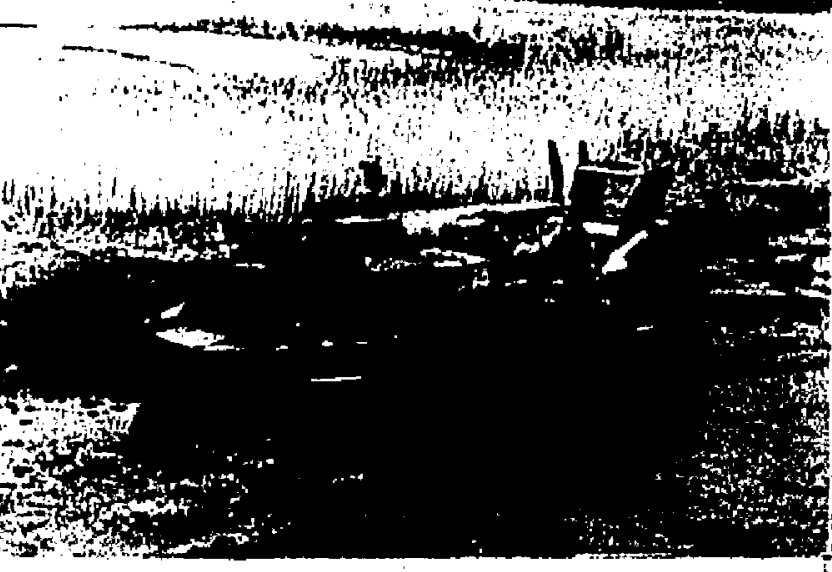

(a) 8 TON ACV
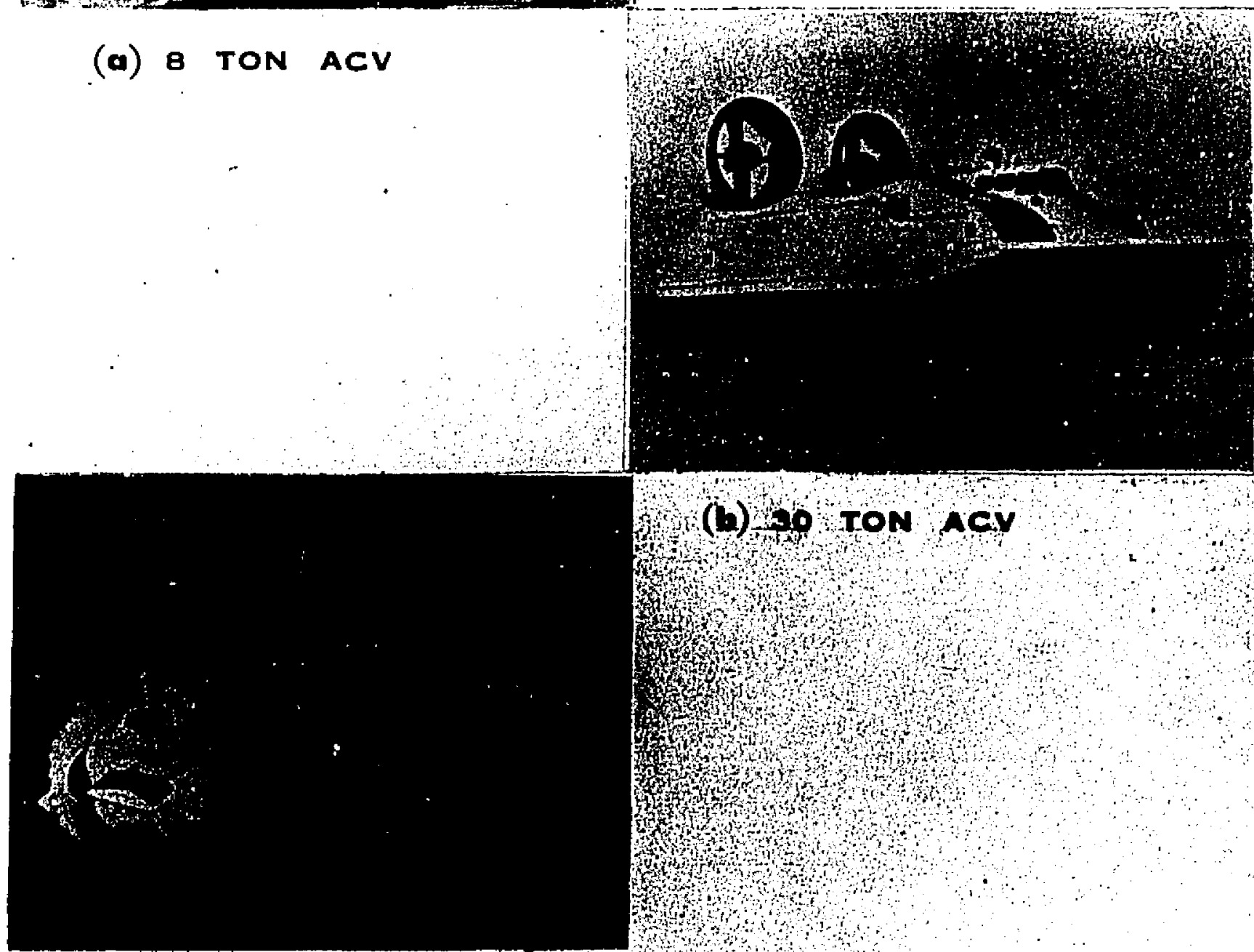

(b) $10, \mathrm{roN} \mathrm{ACy}$

(s) 160 TON ACY 
Cushion Vehicles. A comparison of the three designs is shown in Figure 1-7. The lef figure shows the cross section of a typical plenum chamber with a flexible skirt. The middle figure shows the cross section of a typical ACV peripheral jet trunk. The continuous peripheral nozzle directs the jet inward at a constant angle. In the ACLS trunk show on the right, the jet is formed by many holes which direct the jet at various angles. Consequently, corrections will be necessary in applying existing flow theories developed for simple peripheral jets. These corrections are developed in Chapter 5. 

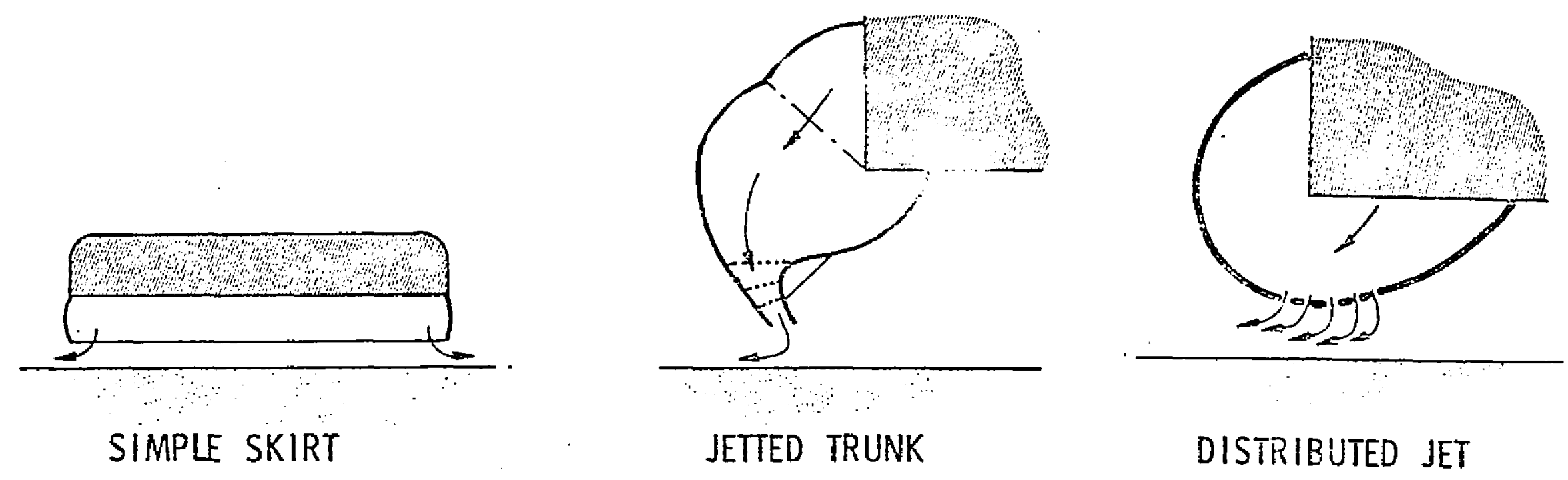
COMPARISON OF AIR CUSHION DESIGNS
FIGURE I-7




\section{PERIPHERAL JET FLOW RELATIONSHIPS}

2.1 Method of Approach to Problem

It is cesired to predict the interrelationship among load capacity, power and jet height for a peripheral jet air suspension system. This problem involves eight independent variables whose values are fixed by the environment, the design, or the mode of operation. There are also eight dependent variables of interest. Consecpuently, it is necessary to develop eight independent equations which relate the eight dependent variables.

The variables of interest and the laws which have been applied to develop the eight equations are summarized in Section 2.2. The development of the equations requires the assumption of a velocity profile across the jet. Several authors have made different assumptions regarding this velocity profile. These different assumptions lead to different theories on the performance of the peripheral jet. The basic relationships which are common to all the theories of interest are developed in Section 2.3. The relationships for specific theories are developed in Sections 2.4 through 2.9 .

\section{$2.2 \quad$ Background}

The Air Cushion Landing System is generally similar in design to Air Cushion Vehicles shown in Figure 1-6. Both employ peripheral jets of the type shown in Figure 1-1(b). However, dhere are differences in the design of the trunk as shown in Figure 1-7. The ACLS uses a distributed jet as compared with a concentrated jet for the Air Cushion Vehicles. The single-peripheral jet system will be considered in this section. Distributed jet systens will be presented in Section 5. 
A number of flow theories have been advanced to predict the plenum pressure which will result from a peripheral jet of a given dosign. (8) These flow theories fall into three general categories.

The first category involves the development of an exact solution of the Navier-Stokes equations of the jet flow. The viscous exact theory developed by Bochler (30) falls into this category. The resulting relationships are quite complicated and therefore only numerical evaluations will yield useful results.

The second category involves the conformal mapping of the hadograph plane for solving the annular jet flow. A number of authors including Chaplin and Stephenson, (31) Strand, (32) Ehrich, ${ }^{(33)}$ Cohen, (34) Bligh, (35) and Roche ${ }^{(36)}$ have developed solutions to the jet flow field, assuming two dimensional, nonviscous flow. These theories have the disadvantage of being overly complex without providing better agreement with experimental results than provided by the simpler theories of category three. $(8,37)$

The third category involves an approximation of the exact solution based upon simplifying assumptions to predict the jet momentum. These theories are known as momentum theories. They have the advantage of providing simple relationships and agreeing ' reasonably well with experimental results. ${ }^{(8,37)}$ A momentum theory which included the effect of viscosity was advanced by Chaplin. (38) However, this analysis requires the assumption of an experimentally developed entrainment function. This approach is considered to have little merit over the application of an experimentally determined coefficient of discharge to a simple nonviscous momentum theory.

The nonviscous momentum theories differ principally in the assumption made for the velocity profile across the jet. The thin jet theory ${ }^{(39)}$ assumes a velocity across the jet which is constant and independent of cushion pressure. It is applicable only for large jet heights or low cushion pressures. The exponential theory ${ }^{(40)}$ assumes an exponential velocity profile across the jet. The Barratt Theory $(41)$ assumes a velocity in the jet which is inversely proportional to the jet radius of curvature. Earl(1) developed a semi-empirical relationship between jet height and velocity so that the predicted flow would be zero at the end point where the jet height is zero. 
Khanzhonkor ${ }^{(42)}$ and Fumita ${ }^{(43)}$ developed separate analyses for suspension systems which employ two peripheral jets to provide a "double seal". Khanzhonkor used the exponential theory and Fumita used the thin jet theory to predict the flow and pressure ratio across each jet.

A number of other authors $(8,37)$ have used the nonviscous momentum theories to predict flow performance of peripheral jet air suspension systems. The momentum theories which have been reported to give the best agreement with test results are the Exponential Theory and the Barratt Theory. ${ }^{(40,37)}$

In the sections to follow, the most prevalent nonviscous momentum theories will be summarized. The development of relationships which are common to all of the peripheral jet theorics are presented in Sections 2.3 and 2.4. The momentum theories devcloped are as follows:

The Thin Jet Theory - Section 2.5,

The Exponential Theory - Section 2.6,

The Barratt Theory - Section 2.7.

The Simple Plenum Theory is presented in Section 2.8. This theory is applicable to the type of air suspension system shown in Figure 1.1(a). The plenum chamber relies upon flow restriction rather than a momentum seal to maintain the overpressure in the plenum.

\subsection{Development of Common Relationships}

\subsubsection{Approach}

In this section, the variables associated with peripheral jet performance are listed, the laws which have been applied are stated, and the relationships which are common to all the peripheral jet theories have been developed. 
The variables involved in the problem are shown on the idealized model of an air cushion landing system in Figure 2-1. These variables may be grouped as follows:

Independent Environmental Variables

$P_{\mathrm{a}} \quad-$ Atmospheric pressure, psfa

$\rho \quad-$ Atmospheric air density $\mathrm{lb} / \mathrm{ft}^{3}$

Independent Design Variables

$A_{c} \quad-$ The effective horizontal area over which cushion pressure acts (cushion area), $\mathrm{ft}^{2}$

$\mathrm{S} \quad-$ Length of the peripheral jet nozzle, $\mathrm{ft}$

$t \quad-$ Width of peripheral jet nozzle gap, $\mathrm{ft}$

$0 \quad-$ Effective nozzle angle, radians

Independent Operating Variables

hp - Energy per unit time contained in air supplied to the jet, horscprower

$W_{A} \quad-$ Weight of aircraft, ib

Dependent Variables

d - Jet height, ft

$\mathrm{J}^{\prime} \quad$ - Magnitude of the reaction imparted by the jet (-Ibs)

$p_{c}\left(P_{C}\right)$ - Cushion pressure, psfg (psi)

$p_{j}\left(P_{j}\right) \quad-$ Trunk (jet) pressurc, psfg (psf)

$P(P) \quad-$ Pressure at an arbitrary point inside the jet, psfg (psf)

$\mathrm{Q}_{\mathrm{j}} \quad-$ Flow rate of air from jet, $\mathrm{ft}^{3} / \mathrm{sec}$

R - Radius of curvature of the path of an infinitesimal element of gas in the jet, $\mathrm{ft}$

$v-$ Velocity of an infinitesimal element of gas insicle the jet, $\mathrm{ft} / \mathrm{sec}$ 


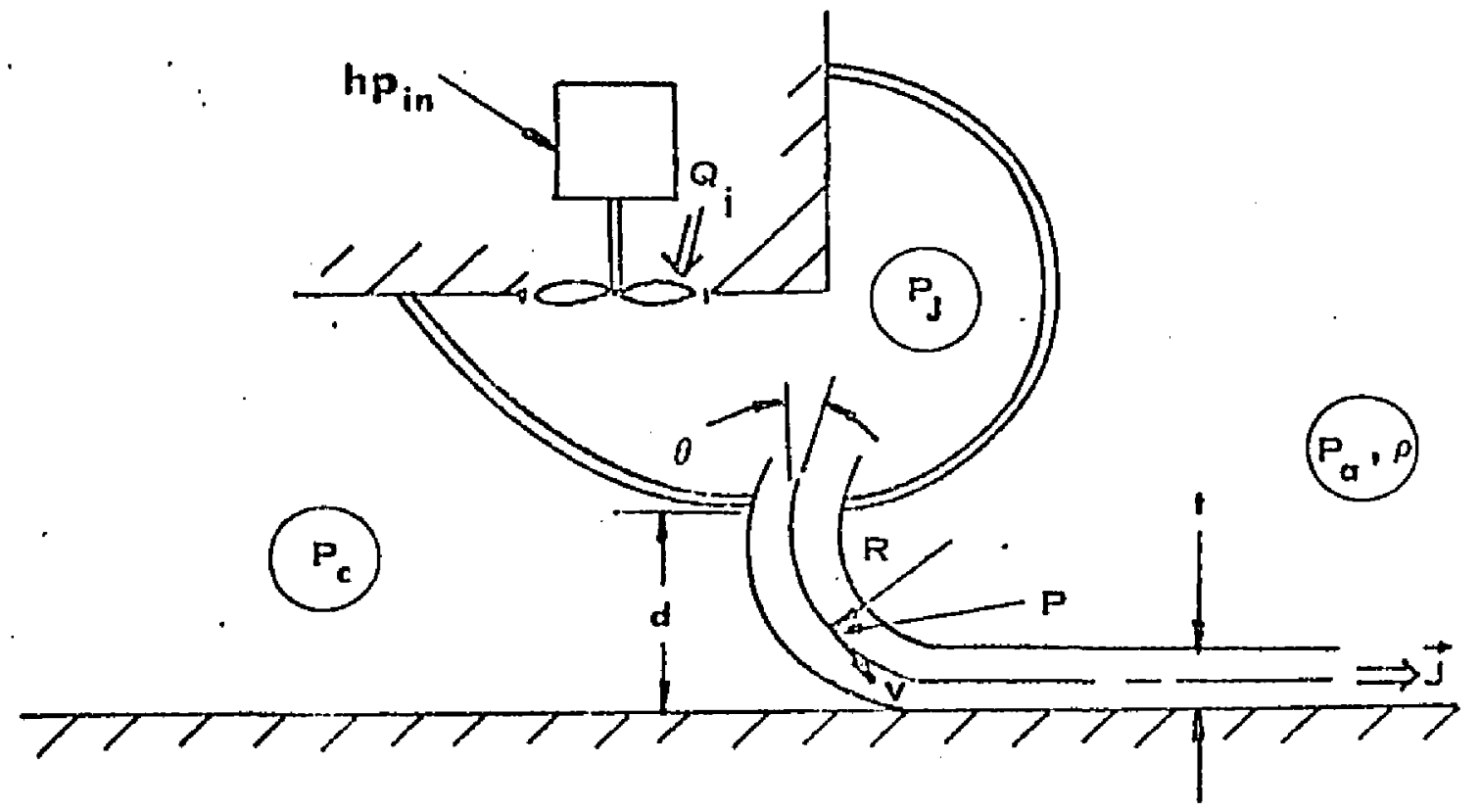

(a) TRUNK CROSS-SECTION

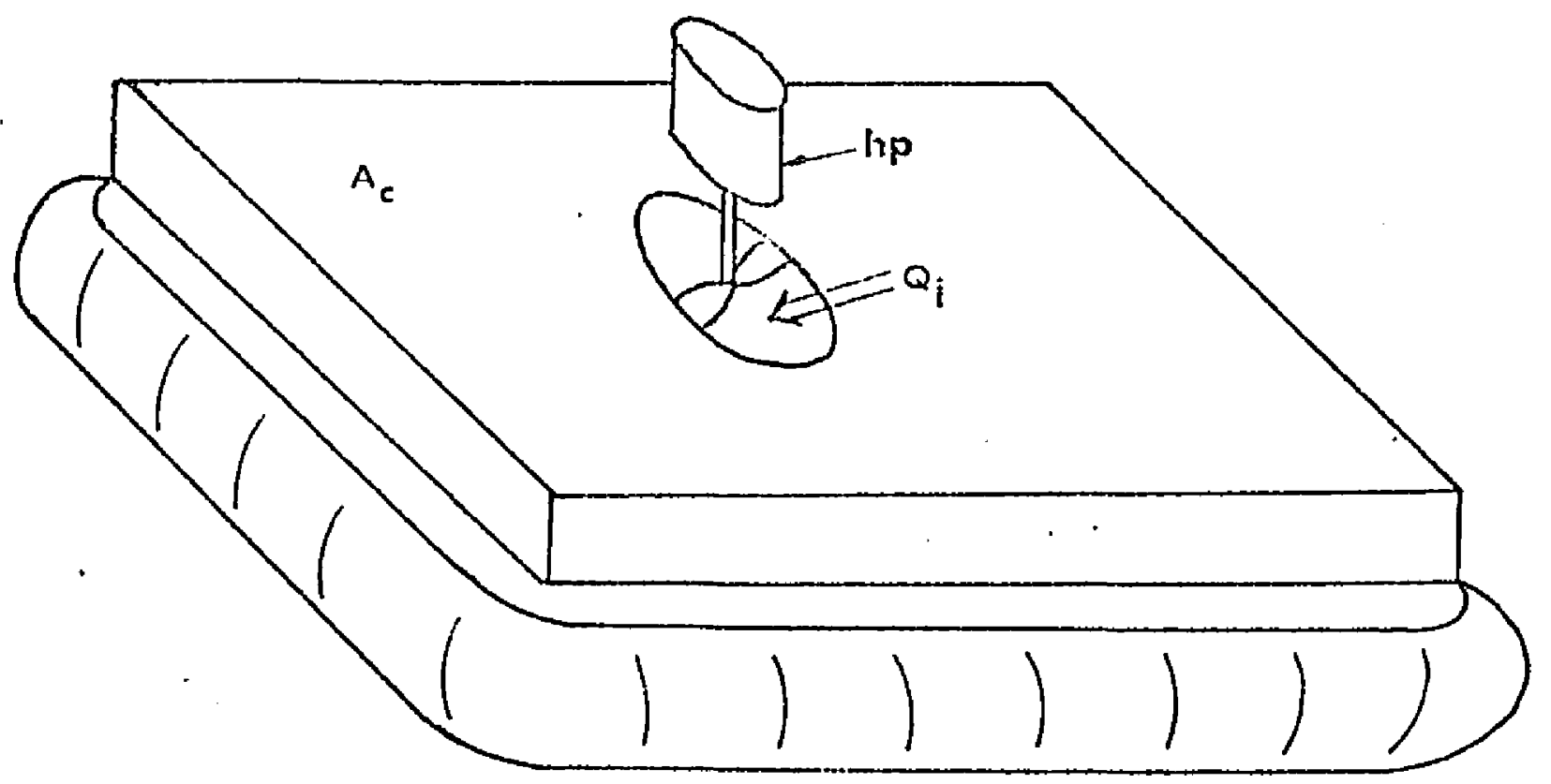

(b) 3 DIMENSIONAL DYNAMIC MODEL

\section{ILLUSTRATION OF MAJOR VARIABLES}

FIGURE 2-1. 
The independent environmental variables are consiclered constants. For a given design, the independent design variables are fixed. It is desired to develop relationships between the independent operating variables and the dependent variables for fixed values of the independent environmental and design variables. Such relationships would allow the prediction of the jet height as a function of power input and aircraft weight. The jet height is an index of the air cushion performance as is discussed in detail in Chapter 9.

If one applies basic laws and principles to a free body of the peripheral jet system, the necessary relationships may be developed. Since there are eight dependent variables, it will be necessary to develop eight independent relationships among the variables.

The relationships are as follows:

(a) Force equilibrium applied at a cross section of the air cushion taken parallel to the ground and at ground level gives:

$W_{A}=f\left(p_{c}, A_{C}\right)$

(b) Conservation of energy involving the energy source for the system gives:

$\operatorname{lip}=f\left(p_{j}, Q_{j}\right)$

(c) Geometric compatibility between the jet radius and the other dimensions gives:

$R=f(d, 0, t)$

(d) D'Alembert's principle applied to an element within the jet gives: $P=f(p, v, R)$

(e) Conservation of energy applied to the jet gives Bernoulli's equation

$$
P_{j}=f(P, v, \rho)
$$


(f) Conservation of mass applied to the jet at its exit plane gives:

$$
Q_{j}=f(v, t)
$$

(g) Force equilibrium applied to the cushion seal gives:

$$
d=f\left(p_{c}, j, 0\right)
$$

(h) The definition of momentum applied to the jet gives:

$$
J^{\prime}=f\left(S, p_{j}, t\right)
$$

The first two equations $(2-1$ and $2 \cdot 2)$ provide relationships among the two independent operating variables and three of the dependent variables. These equations do not involve assumptions concerning the flow in the jet. Consequently, they are applicalble to all of the jet flow theories to be developed later. The approach taken here is to develop these two relationships first, then develop the remaining relationships based upon various theories of flow in thrs jet.

The development of the first two relationships, which are common to all flow theories for the peripheral jet, is presented in Sections 2.3.2 and 2.3.3.

\subsubsection{Force Equilibrium}

Force equilibrium may be applied to the air cushion vehicle at the ground footprint as shown in Figure 1-5(a). The following assumptions are made:

2.3.2.1 The ACLS is symmetric and the opposite sides have identical flow, stiffness and geometric characteristics.

2.3.2.2 The center-of-gravity of the aircraft is directly above the center of the air cushion. 
2.3.2.3 The pressure is equal to $P_{c}$ inside the plenum and equal to $P_{a}$ outside the plenum.

2.3.2.4 All flow into the trunk exhausts through the peripheral jet.

2.3.2.5 The thrust from the peripheral jet is negligible.

Force equilibrium applicd at a cross section of the air cushion taken parallel to the ground and at ground level gives:

$$
W_{A}=P C_{c}
$$

\subsubsection{Conservation of Energy Involving the Power Source}

The conservation of energy law may be applied to the energy supplied to the air. In order to apply this principle, the following assumptions ate made:

2.3.3.1 The air is incompressible.

2.3.3.2 The air is inviscid.

2.3.3.3 Energy losses are negligible.

2.3.3.4 Flow is adiabatic.

2.3.3.5 The air velocity in the trunk may be neglected $\left\langle P_{t}=P_{j}\right.$, where $P_{t}=$ total pressurel.

The work done on the air by the fan must produce an increase in the energy of the air. 


$$
\overline{W_{f}}=\left(P_{j}-P_{a}\right) V_{f}
$$

where: $\quad \bar{W}_{f}$ is the work done by the fan per revolution and

$V_{f}$ is the air volume displaced per revolution.

The above equation may be differentiated with respect to time.

$$
\frac{d \bar{W}_{f}}{d t}=p_{j} \frac{d V_{f}}{d t}
$$

Written in terms of horsepower input to the air, the relationship becomes:

$$
h p=\frac{P_{j} Q_{j}}{550}
$$

\subsection{General Technique for Developing Flow Relationships}

\subsubsection{Approach}

In this scction, the assumptions required to develop the flow equations are listed and the general flow equations are developed. All the assumptions stated in this section apply to all peripheral jet theories developed by this author in Sections 2.5 through 2.8. Each of the theories also has additional assumptions peculiar to the particular theory. The various laws will be applied in the same order as will be used in the sections to follow.

\subsubsection{Geometric Compatibility}

The various theories differ somewhat with respect to the assumptions made in the area of geometric compatibility. The particular assumption for the geometry of the jet will be considered separately for each of the theories to follow. It will be shown later that a convenient dimensionless ratio associated with the nozzle geometry can be defined and will 
be referred to as the jet thickness parameter. This parameter is represented by the symbol $X$ and is defined as follows:

$$
x=\frac{t}{d}(1+\sin 0)
$$

2.4.3 D'Alembert's Principle Applied to the Jet

A relationship involving the pressure, the velocity and the radius of curvature of the jet may be obtained by. apslying d'Alembert's principle.

The following assumptions are applicable:

2.4.3.1 The viscosity is negligible.

2.4.3.2 The density of the gas is constant.

2.4.3.3 The pressure and velocity along any streamline is constant.

D'Alembert's principle may be applied in the $R$ direction to the infinitesimal element of gas shown in Figure 2-2. The resulting equation is:

$$
\frac{v^{2}}{R} d m=(P+d P)\left(R+\frac{d R}{2}\right) d \eta-2\left(P+\frac{d P}{2}\right) \sin \frac{d \eta}{2} d R-P\left(R--\frac{d R}{2}\right) d \eta
$$

The above equation may be simplified by eliminating third order differentials and introducing the following substitutions:

$$
\begin{aligned}
& \frac{d \eta}{2} \approx \sin \frac{d \eta}{2} \\
& \frac{\rho}{g_{0}} d R d \eta=d m
\end{aligned}
$$




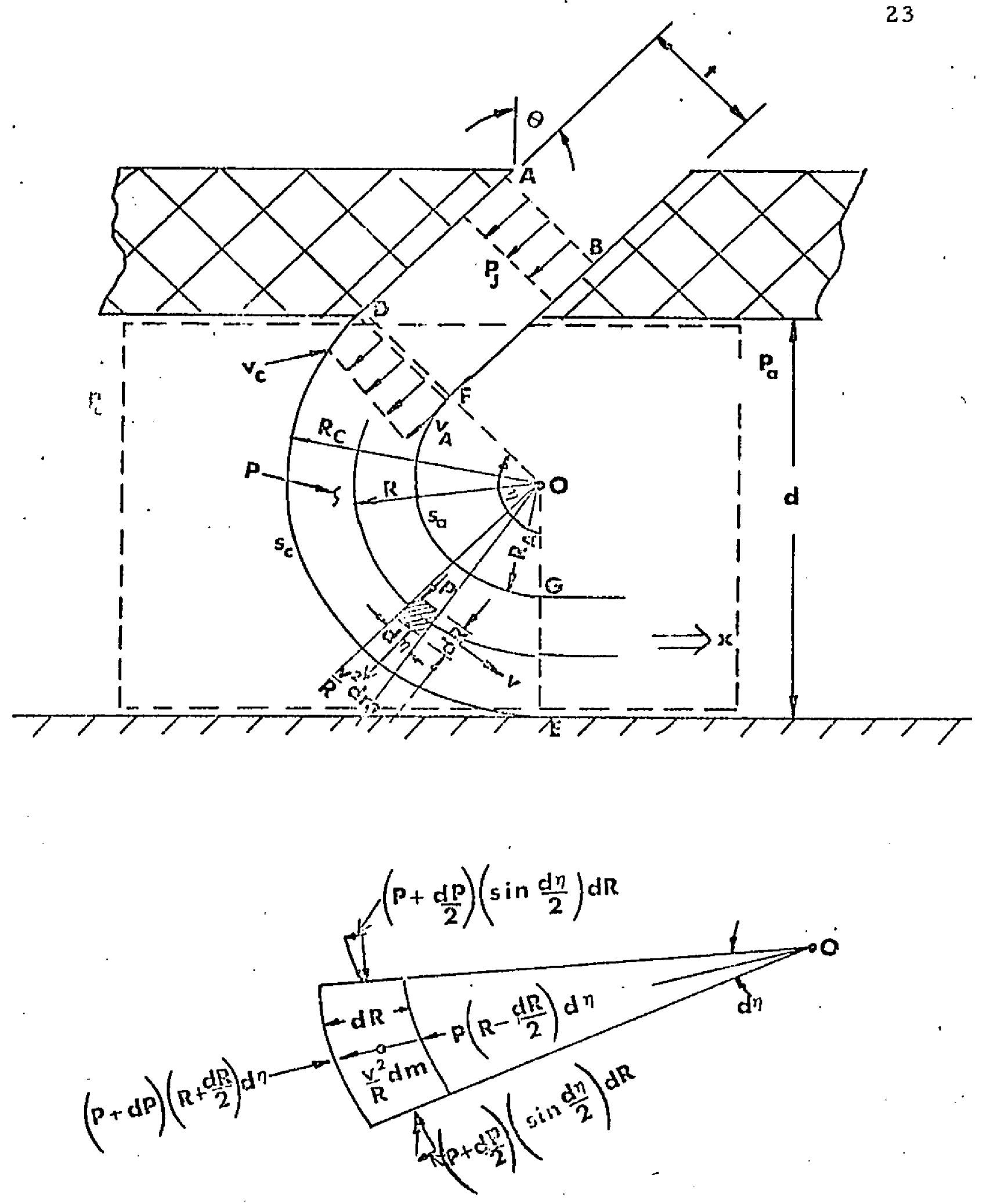

MODEL FOR GENERAL THEORY

FIGURE 2-2 
The resulting equation becomes:

$$
\frac{\rho v^{2}}{g_{o} R} d R d \eta=d P d \eta
$$

Since $d \eta \neq 0$ it is possible to divide by $d \eta$ to give a simple differential equation which relates the pressure at any point in the jet to the velocity and the radius of curvature at that point. The equation is:

$$
d P=\frac{\rho}{g_{0}} v^{2} \frac{d R}{R}
$$

\subsubsection{Conservation-of-Energy Applied to Jet}

A redationship between the pressure and velocity at any point in the jet may be obtained by applying conservation of energy.

The following assumptions are applicable:

2.4.4.1 The air is incompressible.

2.4.4.2 The air is inviscid.

2.4.4.3 Energy losses are negligible.

2.4.4.4 The flow is adiabatic.

2.4.4.5 The air velocity in the trunk may be neglected.

2.4.4.6 The total pressure is everywhere constant.

2.4.4.7 The air velocity in the trunk is equal to zero and the pressure $P_{j}=P_{t}$ (where $P_{1}=$ total pressure). 
2.4.4.8 The flow velocity is perpendicular to the exit plane DF.

2.4.4.9 The effect of change of height of the gas is negligible.

2.4.4.10 The energy along any streamline is constant.

The conservation of energy principle may be applied to an arbitrary streamline(s) in the jet shown in Figure 2-2. The energy of the gas at any point in the trunk must equal the energy of the gas at any point in the streamline. Since there is negligible heat transfer, work, frictional loises, gas compression, and chango in height during the flow process, the energy balance becomes:

$$
\frac{P_{j}}{\rho}+\frac{v_{j}^{2}}{2 g_{0}}=\frac{P}{\rho}+\frac{v^{2}}{2 g_{o}}
$$

In the above equation, the $\mathbf{j}$ subscripts denote any point in the trunk and the variables which are not subscripted denote any point in the jet. Assumption 2.4.4.5 permits the elimination of the $v_{j}{ }^{2}$ from the above equation. The resulting equation is:

$$
\frac{P_{j}}{\rho}=\frac{P}{\rho}+\frac{v^{2}}{2 g_{0}}
$$

Equation (2-13) gives a relationship between the trunk pressure and the pressure and velocity at any point in the jet.

\subsubsection{Conservation of Mass}

A relationship involving the flow may be obtained by summing the increments of flow across the jet. The assumptions in applying this principle are the same as those for the conservation-of-energy principle. These assumptions are listed in Section 2.4.4. A model for 
the jet flow is shown in Figure 2-2. For an arbitrary value of $\eta$, the increments of flow across the jet may be summed in the radial direction. The resulting equation is:

$$
Q_{j}=s \int_{R_{a}}^{R_{c}} y d R
$$

In the above equation, the integration is performed with $\eta=$ constant. The variable $S$ is the length of the jet curtain. Equation (2-14) gives the total flow from the jat, evaluated at any angle $\eta$. It is generally convenient to evaluate the flow at the exit plane where $\eta=90^{\circ}+0$

\subsubsection{Force Equilibrium Applied to the Jet Seal}

Force equilibrium may be applied to the peripheral jet seal shown in Fig!.le 2.2 . The assumptions from the previous sections are retained. The following assumptions are added:

2.4.6.1 The surfaces above and below the air cushion are rigid and impervious.

2.4.6.2 The cushion is in static equilibrium (no air entering or leaving the cushion).

2.4.6.3 The cushion pressure is separated from the atmosphere by a peripheral jet.

2.4.6.4 The mixing between the jet and the surrounding environment is negligible and the velocity profile is constant along the length of the jet (two dimensional flow). 
2.4.6.5 The total momentum of the jet at the nozzle exit plane (Section DF, Figure 2-2) is equal in magnitude to the total momentum of the jet at the cushion exit plane (Section EG, Figure 2-2).

Uncler equilibrium conditions, air neither enters nor leaves the cushion (plenum). The cushion pressure is maintained by the reaction which results from the momentum change in the peripheral jet. For forcc equilibrium in the air gap (d), the cushion pressure times the area over which it acts must equal the time rate of change of the total jet momentum. The equation expressing force equilibrium across the air gap in the direction perpendicular to the air gap (the $x$ direction) is:

$$
P_{\mathrm{c}} S d=\frac{d}{d t}(\vec{J})_{\times} \frac{1}{g_{0}}
$$

The magnitude of the force in the $x$ direction developed by the change in momentum of the gas may be determined by the momentum principle applied to the control volume. The momentum principle may be stated:

$$
F_{x}=\frac{d}{d t}(J)_{x} \frac{1}{g_{0}}=\frac{1}{g_{0}}\left[\frac{d\left(w v_{x}\right)}{d t}+\sum_{\text {out }} w v_{x}-\sum_{\text {in }} w v_{x}\right]
$$

If the velocity and flow rate are assumed constant, and the geometry of Figure 2.2 is applied, the resulting cquation is

$$
\frac{d}{d t}(J)_{x} \frac{1}{g_{0}}=J^{\prime}(1+\sin 0)
$$

where

$$
\mathrm{J}^{\prime} \equiv \frac{\mathrm{wv}}{\mathrm{g}_{\mathrm{o}}}
$$




\subsubsection{Pressure Variation Across the Jet}

The principal difference between the various momentum theories is a difference in the pressure variation across the jet. All theories presented assume the pressure and velocity along any streamline is constant (Assumption 2.4.3.3). Consequently, jet pressure is independent of $\eta$ in Figures 2-2, 2-3, and 2-4.

The pressure variation across the jet may be determined by combining the conservation-of-energy equation, Equation (2-13), and the D'Alembert's equation, Equation (2-12). The result is:

$$
\frac{d P}{P-P_{j}}=-2 \frac{d R}{R}
$$

The resulting differential equation gives the pressure variation with radius. This equation may be integrated between the jet boundary and some arbitrary radius to give the pressure at any point inside the jet.

The pressure variations for the three momentum theories are presented in Sections 2.5.7, 2.6.7, and 2.7.7.

\subsubsection{Velocity Variation Across the Jet}

The velocity variation across the jet may be found in a similar manner to the pressure variation. In this case, the pressure terms in the D'Alembert's Principle relationship, Equation (2-12), may be eliminated by substitution of the conservation-of-energy relationship, Equation (2.13). The result is:

$$
\frac{d v}{v}=\frac{-d R}{R}
$$


The resulting differential equation relates the velocity variation to the radius. The equation may be integrated between the jet boundary and some arbitrary radius vector with terminus inside the jet to give the velocity at any point inside the jet. As a consequence of Assumption 2.4.3.3 the velocity in the jet is independent of $\eta$ in Figures 2-2, 2-3, and 2-4.

The velocity variations for the three momentum theorics are presented in Sections 2.5.8, 2.6.8, and 2.7.8.'

\subsubsection{Momentum}

The magnitude of the total reaction of the jet at the nozzle exit plane (Section DF, Figure 2-2), may be deiernined by summing the total mass flow rate and velocity across Scction DF. The mass flow rate is determined by summing all the flow across section DF. The result is

$$
w=\rho S \int_{R_{a}}^{R_{c}} v d R
$$

The integration is performed at Section DF. This section is specified by holding the angle $\eta$ constant at $90^{\circ}+0$. By applying the definition of momentum, Equation (2-16), and by using the mass-flow-rate relationship developed above, an expression for the magnitude of the total jet reaction may be developed.

$$
J^{\prime}=\frac{\rho}{g_{0}} S \int_{R_{a}}^{R_{c}} v^{2} d R
$$

The integration is performed with $\eta=$ constant $=90+0$.

Equation (2-17) gives the magnitude of the total reaction of all the air escaping from the jet at the bottom of the trunk, evaluated at the nozzle exit plane at the lower surface of the trunk. 


\subsubsection{Jet Flow}

The different momentum theories predict different flows as a consequence of the different pressure distributions assumed to exist across the jet. The total jet flow, $a_{j}$, may be found by integrating Equation (2-14). This integration has been performed in Sections 2.5.10, 2.6.10, and 2.7.10. In each section, the final result has been arranged so that the expressions for the different theories may be compared easily. In each case, the expression for flow has the following form:

$$
\mathrm{O}_{j}=\mathrm{tS} \sqrt{\frac{2 g_{\rho}}{\rho}\left(p_{j}\right)} \quad \mathrm{f}\left[\left(\mathrm{p}_{\mathrm{c}} / \mathrm{P}_{\mathrm{j}}\right), X\right]
$$

The term in brackets, if any, signifies the difference between the flow predicted by the three theories. In later sections this term is treated as a flow coefficient and designated $\mathrm{C}_{\mathrm{Q}}$.

\subsubsection{Recovery Pressure Ratio}

The final relationship desired is the ratio of the cushion pressure to trunk pressure as a function of the jet thickness parameter. This relationship has the form

$$
p_{c} / p_{j}=f(X)
$$

where $x \equiv t / d(1+\sin \theta)$. A second relationship between $p_{c} / p_{j}$ can be developed by combining Equations (2-9) and (2-10). The result is:

$$
p_{c} / p_{j}=\left(\frac{W_{A}}{h p}\right)\left(\frac{Q_{j}}{A}\right) \frac{1}{550}
$$

It is evident from the above relationships that $p_{c} / p_{j}$ forms an important link in relating the independent variables $W_{A}$ and hp to the resulting jet height $d$. 
The $\mathrm{p}_{\mathrm{c}} / \mathrm{p}_{\mathrm{j}}=f(X)$ relationships for the three momentum theories have been developed in Sections 2.5.11, 2.6.11, and 2.7.11. The relationships involving aircraft weight $\left(W_{A}\right)$, horsepower (hp) and jet height (d) have been developed in Chapter 3.

\section{$2.5 \quad$ The Thin Jet Theory}

2.5.1 Approachi and Assumptions

In Section 2.3.2, Equation (2-9) was developed which relates aircraft weight to cushion pressure and area

$$
W_{A}=p_{c} A_{c}
$$

In Section 2.3.3, Equation (2-10) was developed which relates input power to trunk pressure and flow.

$$
h p=\frac{p_{j} a_{j}}{550}
$$

It is evident that if a relationship between $p_{c}$ and $p_{j}$ could be determined, and if $Q_{j}$ could be expressed in terms of $p_{c}$ and $p_{j}$, then the aircraft weight and input horsepower could be directly related.

A number of theories have bcen presented in the literature for relating $\mathrm{p}_{\mathrm{c}}$ and $\mathrm{p}_{\mathrm{j}}$. The simplest of these theories is the thin jet theory which is developed in this section. The objective is to determine the flow $O_{j}$ and the $p_{c} / p_{j}$ relationslip which can be used to link Equations $(2 \cdot 9)$ and $(2 \cdot 10)$.

The Thin Jet Theory advanced by Chaplin (39) assumes that the jet height is very much larger than the nozzle thickness $(d \gg t)$. Under these conditions, the jet is extremely thin and is considered as a single streamline (see Figure 2-3). In addition to the assumptions made in Section 2.1, the following restrictions are imposed: 

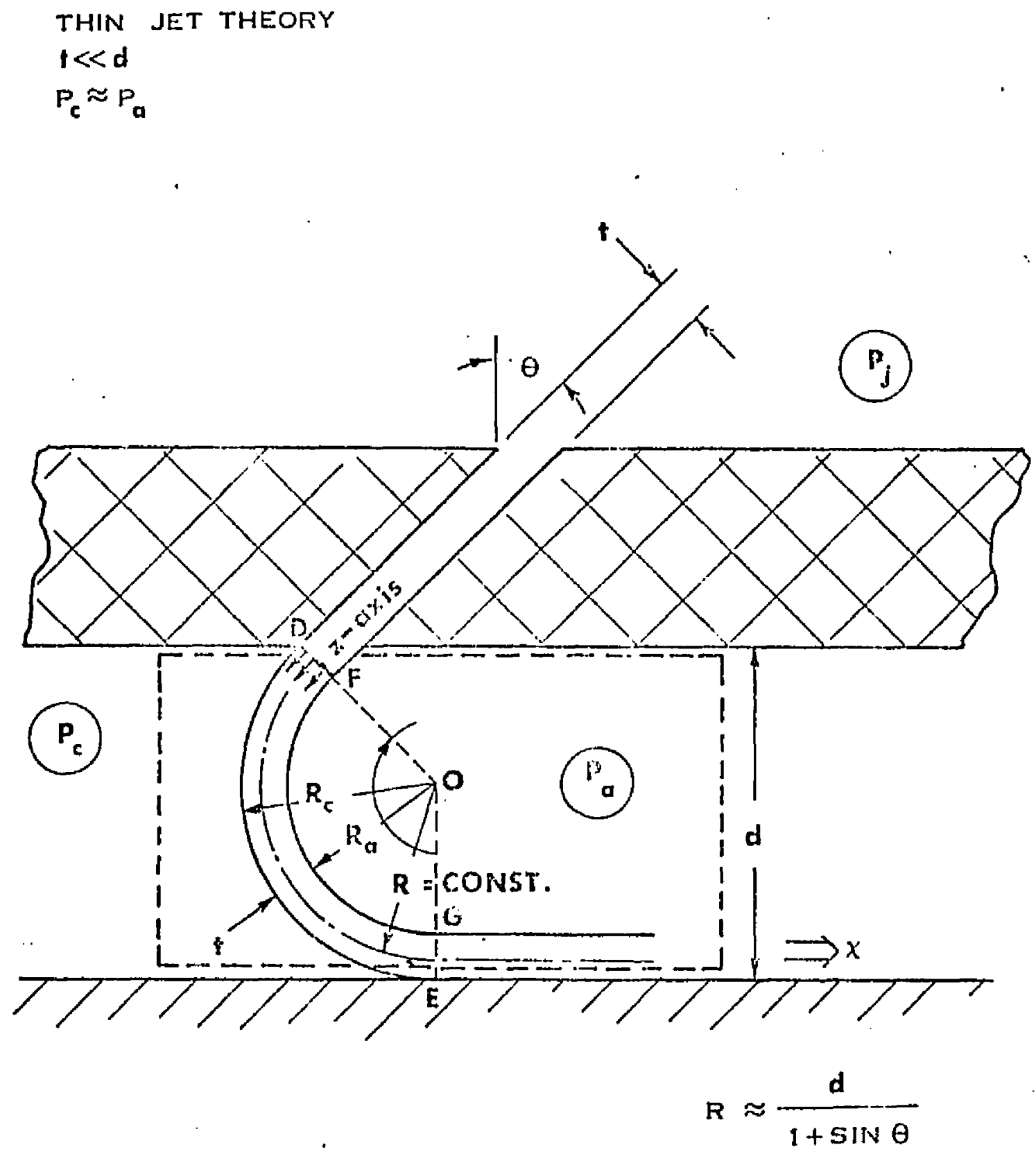

MODEL FOR THIN JET AND EXPONENTIAL THFORIES FIGURE 2-3 
2.5.1.1 The radius $\mathrm{R}$ is constant in magnitude.

2.5.1.2 The velocity and pressure variations are linear across the jet.

2.5.1.3 The increments $d P$ and $d R$ in Equations (2-12), (2-14), and (2-17) may be replaced by the finite quantities:

$$
\Delta P=P_{c}-P_{a} \text { and } \Delta R=t
$$

2.5.1.4 The streamline is tangent to the ground at Section EG of Figure 2-3.

2.5.1.5 The thickness of the jet is sufficiently small such that $R_{c}=R=R_{a}$.

2.5.1.6 The pressure and velocity along the streamline from DF to EG is constant (Figure 2-3).

2.5.1.7 The pressure variation across the jet is assumed to be linear and the average pressure may be expressed by the relation:

$P=P_{a}+f\left(P_{c}-P_{a}\right)$

where $0 \leqslant f \leqslant 1$. Therefore, $P_{c} \geqslant P \geqslant P_{a}$. The value of $f$ may be determined experimentally. Chapman ${ }^{(39)}$ suggests the use of $f=0$. Stanton-Jones $(40)$ and Gates $(4,31)$ have developed theories using a value of $f=1$. For the purposes of this development, $f=0$ will be considered. 


\subsubsection{Geometric Compatibility}

From Figure 2-3 it may be seen that the following geometric relationship holds:

$$
R=\frac{d}{1+\sin \theta}
$$

\subsubsection{D'Alembert's Principle}

Assumption 2.5.1.3 applied to the D'Alembert's equation, Equation (2-12), gives:

$$
\mathrm{p}_{\mathrm{c}}=\left(\frac{\rho}{\mathrm{g}_{\mathrm{o}}}\right)\left(\mathrm{v}^{2}\right)\left(\frac{\mathrm{t}}{\mathrm{R}}\right)
$$

In the above equation, both $p_{c}$ and $v$ are unknown quantities. The calculation of $p_{c}$ is dependent upon $v$. In turn, $v$ is dependent upon $P$ which is determined by the choice of $f$ in Assurnption 2.5.1.7.

\subsubsection{Conservation of Energy}

Conservation-of-energy applied as specified in Section 2.4 .4 gives:

$$
P_{j}=P+1 / 2\left(\frac{\rho v^{2}}{g_{0}}\right)
$$

2.5.5 Conservation of Mass

Conservation-of-mass applied as specified in Section 2.4 .5 together with Assumption 2.5.1.3 gives:

$$
\mathrm{o}_{\mathrm{j}}=\mathrm{st} \mathrm{v}
$$

\subsubsection{Force Equilibrium}

Force equilibrium applied as specified in Section 2.4.6, together with Assumptions 2.5.1.1, 2.5.1.4, 2.5.1.6, and the Geometric Compatibility Assumption, Equation (2-19), gives: 


$$
p_{c} d S=J^{\prime}(1+\sin 0)
$$

\subsubsection{Pressure Variation}

The pressure variation across the jet is constant and equal to the value assumed in Assumprition 2.5.1.7,

$$
\mathrm{P}=\mathrm{P}_{\mathrm{a}}
$$

2.5.8 Velocity in the Jet

The velocity in the jet may be determined by substituting the pressure in the jet, Equation (2-24), into the conservation-of-energy relationship, Equation (2-21). The result is:

$$
v=\sqrt{\frac{2 g_{o}}{\rho}\left(p_{j}\right)}
$$

2.5.9 Momentum

The reaction of the jet may be determined by combining Equations (2-16), (2-22), and (2-25). The result is:

$$
J^{\prime}=2 S p_{j} t
$$

2.5.10. Jet Flow

The flow may be determined by combining the encrgy and mass-conservation equations, Equations (2-21) and (2-22), and applying the pressure equation, Equation (2-24). The result is:

$$
\mathrm{o}_{\mathrm{j}}=\mathrm{st} \sqrt{\left(\frac{2 \mathrm{~g}_{\mathrm{o}}}{\rho}\right)\left(\mathrm{p}_{\mathrm{j}}\right)}
$$




\subsubsection{Pressure Ratio}

The pressure ratio for the system may be obtained by combining the equilibrium and momentum equations, Equations (2-23) and (2-26), and applying the definition for jet thickness parameter, $x=t / d(1+\sin \theta)$, Equation (2-11). The result is:

$$
\mathrm{P}_{\mathrm{c}} / \mathrm{p}_{\mathrm{j}}=2 \mathrm{X}
$$

\section{$2.6 \quad$ The Exponential Theory}

\subsubsection{Approach and Assumptions}

The simplest theory for relating $\mathrm{p}_{\mathrm{c}} / \mathrm{p}_{\mathrm{j}}$ to jet geometry was presented in Section 2.5. In the present section, a more accurate theory has been developed. The divelopment presented follows the overall approach outlined in Section 2.4. The objective of tiris section is to develop a more exact relationship between $p_{c}$ and $p_{j}$ so that input horsepower, Equation (2-9), and aircraft weight, Equation (2-10), can be dircctly related.

The exponential theory was advanced by Stanton-Jones. ${ }^{(40)}$ In this theory, the pressure variation across the jet is exponential as shown in Equation (2-3\%). The additional assumptions are:

2.6.1.1 The radius $R$ is constant and can be approximated by $R_{c}$.

2.6.1.2 The radius $R_{c}$ is tangent to the ground at Section EG of Figure 2-3.

\subsubsection{Gcometric Compatibility.}

The geometric compatibility assumptions are based upon Figure 2-2. It may be seen that the following relationships hold:

$$
R_{c}=\frac{d}{1+\sin \theta}
$$




$$
R_{c}=R_{a}+t
$$

\subsubsection{D'Alembert's Principle}

Assumption 2.3.3.1 applied to the $D^{\prime}$ Alembert equation (2-12) gives:

$$
\int_{P_{a}}^{P_{c}} \frac{d P}{v^{2}}=\frac{p}{g_{o} R_{a}} \int_{R_{a}}^{R_{c}} d R
$$

The variables of integration in the above equation may be changed to eliminate the $R_{c}$ and $R_{a}$ variables. The integration is performed along the $z$ axis lat Section $D F$ in Figure 2-3) between $z=0$ and $z=t$. By applying the new dummy variable, $z$, and using Equations (2-29) and (2-11), the $A$ varialse may be eliminated from Equation (2-31). The result is:

$$
\int_{P_{a}}^{P_{c}} \frac{d P}{v^{2}}=\frac{\rho}{g_{0}} \frac{x}{t} \int_{0}^{t} d z
$$

\subsubsection{Conservation of Energy}

Conservation of energy applied as specified in Section 2.4.4 gives:

$$
v^{2}=\frac{2 g_{0}}{\rho}\left(P_{j}-P\right)
$$

\subsubsection{Conservation of Mass}

Conservation of mass may be applied by integrating the velocity across the $z$-axis between $z=0$ and $z=t$ as shown in Figure 2-3.

$$
a_{j}=s \int_{0}^{t} v d z
$$


2.6.6 Force Equilibrium

Force equilibrium applied as specified in Section 2.4.6, combined with Assumptions 2.6.1.1 and 2.6.1.2, gives:

$$
P_{c} S d=J^{\prime}(1+\sin \theta)
$$

\subsubsection{Pressure Variation}

The velocity ralationship, Equation (2-33), substituted into the D'Alembert equation (2-32) between the outer boundary and some arbitrary point (z) inside the jet gives:

$$
p=p_{j}\left(1-e^{-2 \times z / t}\right)
$$

where $X$ is defined by Equation $(2 \cdot 11)$.

\subsubsection{Velocity in the Jet}

The velocity in the jet may be determined by solving the pressure variation, Equation (2-36), with the energy equation, Equation (2-33). The result is:

$$
v=\sqrt{\frac{2 \mathrm{~g}_{\mathrm{O}}}{\rho} \mathrm{p}_{\mathrm{j}}}\left(\mathrm{e}^{[-\mathrm{X} z / \mathrm{t}]}\right)
$$

\subsubsection{Momentum}

The total reaction of the jet may be determined by Equation (2-17).

$$
J^{\prime}=\rho \frac{S}{g_{0}} \int_{0}^{t} v^{2} d z
$$


Substituting in Equation (2-37) and integrating gives:

$$
J^{\prime}=2 t S p_{j}\left[\frac{1}{2 x}\left(1-e^{-2 x}\right)\right]
$$

2.6.10 Jet Flow

The jet flow may be determinod by combining the velocity relationship, Equation (2-37), with the conservation of mass equation, Equation (2-34), and integrating. The result is:

$$
Q=t S \sqrt{\frac{2 g_{O}}{\rho}\left(P_{j}\right)} \frac{1}{x^{[}}\left[\left(1-e^{-x}\right)\right]
$$

\subsubsection{Pressure Ratio}

The pressure ratio may be determined from the force equilibrium relationship, Equation (2-35), combined with the momentum relationship, Equations (2-38) and (2-11). The result is:

$$
p_{c} / p_{j}=1-e^{-2 x}
$$

2.7 The Barratt Theory

\subsubsection{Approach and Assumptions}

The Barratt theory has been reported to provide quite accurate predictions of the performance of a peripheral jet. ${ }^{(40,37)}$ In this section, the jet flow and recovery pressure ratio predicted by the Barratt theory have been developed. These parameters are related to aircraft weight and horsepower in Chapter 3. 


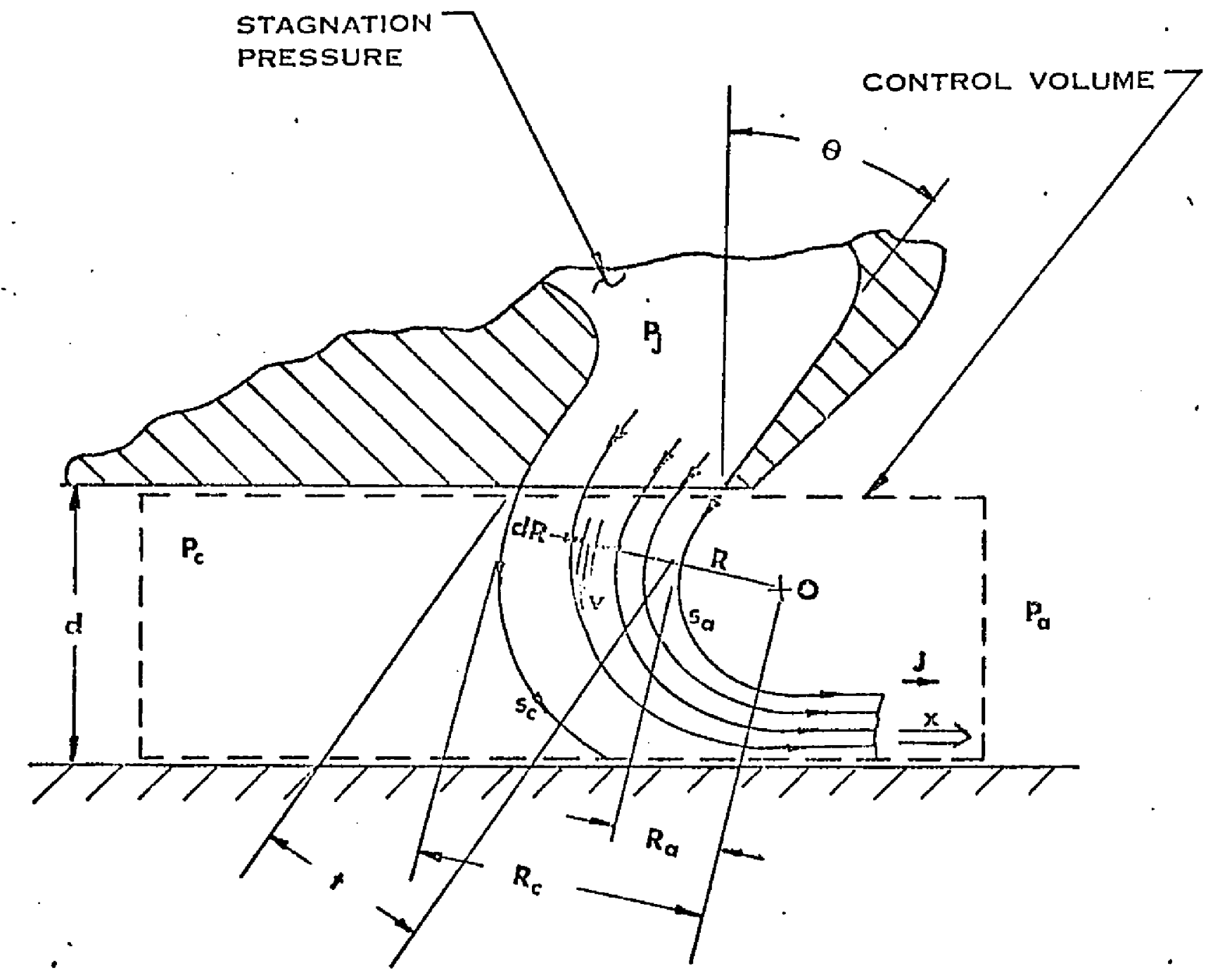


Barratt's theory ${ }^{(41)}$ differs from the previous theories in the geometry assumed for the jet. A cross section of the jet is shown in Figure 2-4. It should be noted that in this theory it is not necessary for the jet thickness to be constant and streamline, $\mathrm{s}_{\mathrm{c}^{\prime}}$ does not have to be tangent to the ground.

In addition to the assumptions made in Sections 2.3 and 2.4 , the following assumptions are made: .

2.7.1.1 At the jet exit plane all streamlines have a common center of curvature (shown as point $M$ in Figure 2-4).

2.7.1.2 The total head or stagnation pressure is constant across the jet.

2.7.1.3 The total momentum $J$ of the jet after the jet has been deflected is equal in magnitude to the exit plane jet momentum.

2.7.1.4 The pressure along any streamline is constant.

\subsubsection{Geometric Compatibility}

From the geometry in Figure 2.4 it may be seen that at Section DF

$$
R_{c}=R_{a}+t
$$

Based upon Assumption 2.7.1.3 it is possible to use geometric compatibility to calculate the change in momentum of the jet. The angle through which the jet turns is $90^{\circ}+$ 0 . The net change of the momentum vector may then be written:

$$
\frac{d}{d t}(\vec{J})_{x}=J^{\prime}(1+\sin \theta) g_{0}
$$




\subsubsection{D'Alembert's Principle}

D'Alembert's Principle applied as specified in Section 2.4.3 gives:

$$
\frac{d P}{d R}=\frac{\rho_{v}^{2}}{R g_{0}}
$$

\subsubsection{Conservation of Energy}

The conservation-of-energy principle applied as specified in Section 2.4 .4 gives:

$$
P_{i}=P+\frac{\rho v^{2}}{2 g_{0}}
$$

In order to determine the velocity variation across the jet, it is desired to replace $d P / d R$ in Equation (2-43) with an expression for $d v / d R$. The needed expression may be derived by differentiating the energy equation (2-44) with respect to $R$ and applying Assumption 2.7.1.2.

$$
\frac{\partial P}{\partial R}=-\frac{\rho}{g_{0}} v \frac{d v}{d R}
$$

Equations (2.45) and (2.43) have been combined in Equation (2-5.1).

\subsubsection{Conservation of Mass}

The conservation-of-mass principle applied as specified in Section 2.4.5 gives:

$$
a_{j}=s \int_{R_{a}}^{R_{c}} v d R
$$




\subsubsection{Force Equilibrium}

Force equilibrium applied as specified in Section 2.4 .6 in conjunction with the geometric compatibility relationship developed in Equation (2-4.1) gives:

$$
P_{C} S d=J^{\prime}(1+\sin 0)
$$

\subsubsection{Pressure Variation}

The pressure variation may be found by solving the D'Alembert equation (2-43) for $v^{2}$ and substituting it in the conservation of energy equation (2-44). The result is:

$$
\int \frac{d P}{P-P_{j}}=-2 \int \frac{d R}{R}
$$

At the inside to jet boundary (streamline $s_{c}$ )

$$
\begin{aligned}
& P=P_{C} \\
& R=R_{C}
\end{aligned}
$$

By integrating Equation (2-48) and applying the boundary condition to cvaluate the constant the following equation is obtained:

$$
P=P_{j}+\left(\frac{R_{C}}{R}\right)^{2} \quad\left(P_{c}-P_{j}\right)
$$

At the outside of the jet boundary (streamline $s_{a}$ ),

$$
\begin{aligned}
& P=P_{a} \\
& R=R_{a} \\
& v=v_{a}
\end{aligned}
$$


Equation (2-49) evaluated at the outside boundary gives:

$$
\frac{R_{a}}{R_{c}}=\sqrt{1-P_{c} / P_{j}}
$$

\subsubsection{Velocity Variation}

The velocity variation may be determined by equating the D'Alembert and the cnergy equations as formulated in Equations (2-43) and (2-45) respectively. The result is:

$$
\int \frac{d v}{v}=-\int \frac{d R}{R}
$$

At the cutside jet boundary (streamline $s_{a}$ )

$$
\begin{aligned}
R & =R_{a} \\
v & :=v_{a} \\
P & =P_{a}
\end{aligned}
$$

By integrating Equation (2-51) and applying the boundary conditions, the following equation results:

$$
v=\frac{R_{a}}{R} v_{a}
$$

$v_{a}$ may be expressed in terms of $P_{j}$ by applying $v=v_{a}$ where $P=P_{a}$ in the conservation of energy equation (2-44). The result is:

$$
v_{\mathrm{a}}=\sqrt{\frac{2 \mathrm{~g}_{\mathrm{o}}}{\rho} \mathrm{p}_{\mathrm{j}}}
$$


The last equation may be substituted into the general velocity equation to yield:

$$
v=\frac{R_{\mathrm{a}}}{\mathrm{R}} \sqrt{\frac{2 \mathrm{~g}_{\mathrm{o}}}{\rho} \rho_{\mathrm{j}}}
$$

\subsubsection{Momentum}

The total reaction of the jet may be determined by substituting the value of $v$ given by Equation (2.52) in the momentum equation, Equation (2.17), and integrating between the limits $R_{a}$ and $R_{c}$. The resulting equation is:

$$
J^{\prime}=2 S\left(R_{a}\right)^{2} \jmath_{j} \int_{R_{a}}^{R_{c}} \frac{d R}{R^{2}}
$$

Integration gives:

$$
J^{\prime}=2 S p_{j} \frac{R_{a}\left(R_{c}-R_{a}\right)}{R_{c}}
$$

Equations (2-41) and (2-50) applied to the above relationship give:

$$
J^{\prime}=2 S p_{j} t \sqrt{1-p_{c} / p_{j}}
$$

\subsubsection{Jet Flow}

Jet flow may be determined by substituting the velocity equation $(2-52)$ in the conservation of mass equation (2.46). The resulting equation is:

$$
a_{j}=S R_{a} \sqrt{\frac{2 g_{o}}{\rho}\left(p_{j}\right)} \int_{R_{a}}^{R_{c}} \frac{d R}{R}
$$

Integrating and applying Equations (2-41) and (2-50) gives: 


$$
\mathrm{O}_{\mathrm{j}}=\mathrm{ts} \sqrt{\frac{2 \mathrm{~g}_{\mathrm{o}}}{\rho}\left(\mathrm{p}_{\mathrm{j}}\right)} \frac{\sqrt{1-p_{\mathrm{c}} / p_{\mathrm{j}}}}{1-\sqrt{1-p_{\mathrm{c}} / \mathrm{p}_{\mathrm{j}}}} \log _{\mathrm{e}}\left(1-\mathrm{p}_{\mathrm{c}} / \mathrm{p}_{\mathrm{j}}\right)^{-1 / 2}
$$

\subsubsection{Pressure Ratio}

The pressure ratio may be determined by substituting the momentum equation, Equation (2-54), in the force rquilibrium equation, Equation (2-47), and applying the definition for jet thickness parameter, Equation (2-11), to simplify. The result is:

$$
p_{c} / p_{j}=2 x\left(\sqrt{x^{2}+1}-x\right)
$$

\section{$2.8 \quad$ Plenum Theory}

\subsubsection{Approach and Assumptions}

The relationships cleveloped in Sections $2.4,2.5,2.6$, and 2.7 apply only to a peripheral jet and not to a plenum chamber. In this section, the equations for predicting the horsepower, flow and jet height for a plenum chamber have been developed.

The plenum chamber differs from the peripheral jet as may be observed by comparing Figures 1-1(a) and 1-1(b). In the plenum chamber design, the air is blown clirectly into the plenum (cushion) rather than into the trunk. Consequently, the plenum chamber has no trunk pressure, no peripheral jet, and no momentum seal. The cushion pressure is maintained by the flow restriction imposed by the air gap between the vehicle skirt and the ground. The relationships for this system may be developed by conservation of energy applied to the exit and by conservation-of-mass applied to the air flowing from the power source. The assumptions made in Section 2.3 apply, but those made in Sections 2.4, $2.5,2.6$, and 2.7 do not apply.

The additional assumptions required are:

2.8.1.1 The air is incompressible. 
2.8.1.2 The air is inviscid.

2.8.1.3 Energy losses are negligible.

2.8.1.4 The flow is adiabatic.

2.8.1.5 The air velocity in the cushion may be neglected $\left(p_{t}=p_{c}\right.$, whicre $p_{t}=$ total pressure).

2.8.1.6 The total pressure is everywhere constant.

2.8.1.7 The flow velocity at the exit is two dimensional and perpendicular to the exit plane.

\subsubsection{Conselvation-of-Energy Applied to Exhaust Exit Plane}

The conservation-of-energy equation may be written:

$$
P_{c}=P_{a}+\frac{\rho}{2 g_{o}} v^{2}
$$

Equation (2-58) expresses the cushion pressure in terms of pressure and velocity of the exhaust air which has expanded to atmospheric pressure.

\subsubsection{Conservation of Mass .}

Conservation-of-mass applied to the exhaust exit gives:

$$
a_{p}=v_{p} d_{p} s_{p} C_{d}
$$


where the subscript $\mathrm{p}$ refers to the plenum.

Equation (2-59) expresses the tolal flow from the plenum chamber in terms of the effective flow area and the velocity of the gas crossing the flow area.

2.8.4 Conservation-of-Energy Involving the Power System

Using a development similar to that given in Section 2.3.1, the horsepower delivered to the plerium is:

$$
h p=\frac{p_{c} Q_{p}}{550}
$$

\subsubsection{Determination of Flow}

Flow from the plenum may be olbtained by combining Equations (2-58) and (2-59). The result is:

$$
O_{p}=\sqrt{\frac{P I_{0}}{\rho}\left(b_{c}\right)} c_{d} S_{p} d_{p}
$$

Equation (2-61) gives the total flow from the plenum in terms of the cushion pressure and the effective flow area.

\subsubsection{Horsepower Relationship}

The horsepower input can be determined from Equations (2-61) and (2-60). The result is:

$$
h p=\frac{\left(p_{c}\right)^{3 / 2} s_{p} d_{p} c_{d}}{550}\left(\frac{2 g_{o}}{\rho}\right)^{1 / 2}
$$

Equation (2-62) gives the total horsepower which must be supplied to the air in terms of the custion pressure and the effective flow area. 


\section{COMPARISON OF FLOW THEORIES}

\subsection{Introduction}

In order to make a general comparison of the performance predicted by the flow theories developed in Chapter 2, it is necessary to develop six nondimensional parameters. Three of these parameters are widely used in the literature of Air Cushion Vehicles. These parameters include:

(1) $A_{1}$, the jel augmentation ratio is defined as follows:

$$
A_{1}=\frac{\text { total vehicle lift force }}{\text { reference force }}
$$

A number of different reference forces are used in the literature. ${ }^{(8)}$ In this chapter, the reference force is the thrust which could be generated if the exhaust were discharged vertically downward. The augmentation ratio is discussed in Section 3.7.

(2) $\quad \mathrm{p}_{\mathrm{c}} / \mathrm{P}_{\mathrm{j}}$, the recovery pressure ratio is defined as follows:

$$
\mathrm{p}_{\mathrm{c}} / \mathrm{p}_{\mathrm{j}}=\frac{\text { cushion pressure (gage) }}{\text { trunk pressure (gage) }}
$$

The recovery pressure ratio is discussed in Section 3.2.

(3) $\quad X$, the nozzle thickness parameter which was clefined in Section 2.4 .2 as follows:

$$
x=\frac{t}{d}(1+\sin \theta)
$$


The nozzle thickness parameter is discussed in Section 3.3.

Three additional parameters not found in the literature are also defined in this chapter. These parameters include:

(1)

$\mathrm{C}_{\mathrm{Q}}$, the cushion pressure coefficient is a flow coefficient. This parameter is developed in Secion 3.4.

(2)

$\mathrm{C}_{\text {lit, }}$ the power-thickness parameter, is a dimensionless parameter useful in predicting power requirements for a peripheral jet. This parameter is developed in Section 3.5.

(3)

$\mathrm{C}_{\text {hd }}$, the power-height parameter, is a dimensionless parameter useful in determining the minimum power for a required jet height. This parameter is developed in Section 3.6.

\subsection{Recovery Pressure Ratio}

The ratio of cushion pressure to trunk pressure is known as the recovery pressure ratio. It has been shown previously (Section 2.3 .2 ) that the value of $p_{c}$ may be determined by the aircraft weight and the cushion area. The value of $\mathrm{p}_{j}$ is dependent upon the input power, the jet area, the jet height, and the jet angle. Consequently, the ratio of $p_{c} / p_{j}$ gives an important dimensionless quantity which is dependent on all the major variables. In addition, it will be shown in Chapter 4 that the trunk shape and stiffness are strongly influenced by $\mathrm{P}_{\mathrm{c}} / \mathrm{p}_{\mathrm{j}}$.

Because of the features cited above, $\mathrm{p}_{\mathrm{c}} / \mathrm{p}_{\mathrm{j}}$ was selected as the standard dependent variable against which other dimensionless parameters have been plotted. 


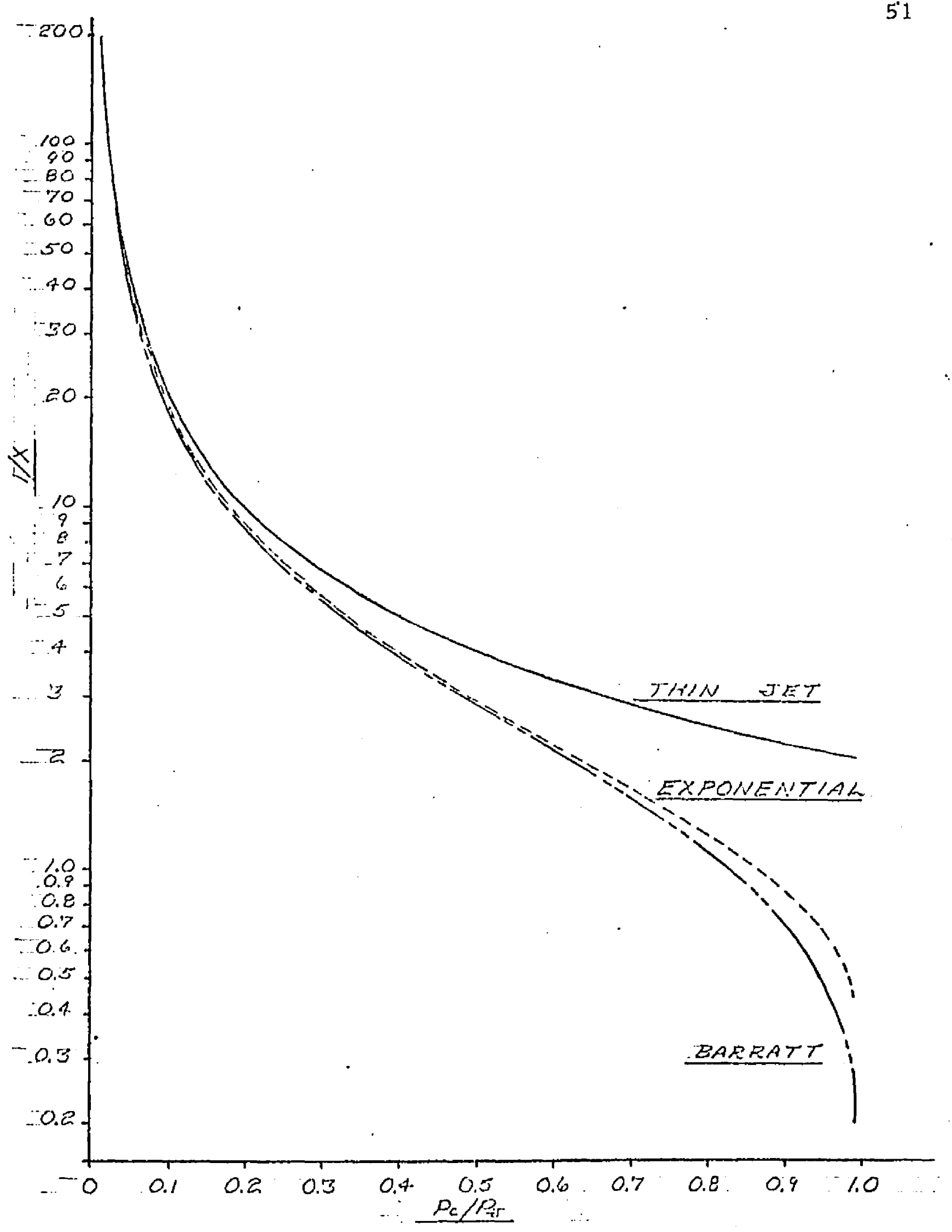

NOZZLE THICKNESS PARAMETER vs $\mathrm{p}_{\mathrm{c}} / \mathrm{p}_{\mathrm{J}}$ FIGURE 3-1 
3.3 Nozzle Thickness Parameter

The no: $:$ le thickness parameter was defined in Section 2.4 .2 as follows:

$$
x=\frac{t}{d}(1+\sin 0)
$$

This parameter relates nozzle geometry to jet height. For a given design, the nozzle thickriess ( $t$ ) and the jet angle ( 0 ) are relatively constant. Equation (2.11) shows that the jet height $(d)$ and the parameter $(X)$ are inversely related. Consequently, the nozzle thickness is valuable in showing the interrelationship between the independent variables and the jet height. This interrelationship has been shown by graphs of various nondimensional parameters plotted against the dependent variable $p_{c} / p_{j}$.

Graphs of $1 / X$ versus $p_{c} / p_{j}$ for the three flow theories are presented in Figure 3-1. The analy/ical relationships between $\left(\mathrm{p}_{\mathrm{c}} / \mathrm{p}_{\mathrm{j}}\right)$ and $\mathrm{X}$ are shown in Table 3.1.

\subsection{Pressure Coefficient}

The pressure coefficient, $\mathrm{C}_{\mathrm{Q}}$, is, in fact, a flow coefficient which is dependent upon the recovery pressure ratio $\left(p_{c} / p_{j}\right)$. This coefficient has been developed in this section.

Consider the total flow from the jet at the nozzle exit plane as shown by Section DF in Figure 2-2. The pressure on the cushion side of the jet is higher than the pressure on the atmospheric side of the jet. Consequently, a velocity and a flow gradient may exist across the thickness of the jet. It is the nature of the assumed pressure gradient across the jet thickness which gives rise to the differences between the three momentum theories. In Sections 2.5.10, 2.6.10, and 2.7.10, expressions have been developed for the total flow from the jet as predicted by the three momentum theories. The resulting equations are:

Thin jet theory

$$
\mathrm{Q}_{\mathrm{j}}=\mathrm{tS} \sqrt{\frac{2 \mathrm{~g}_{\mathrm{o}}}{\rho}\left(\mathrm{P}_{\mathrm{j}}\right)}[1]
$$




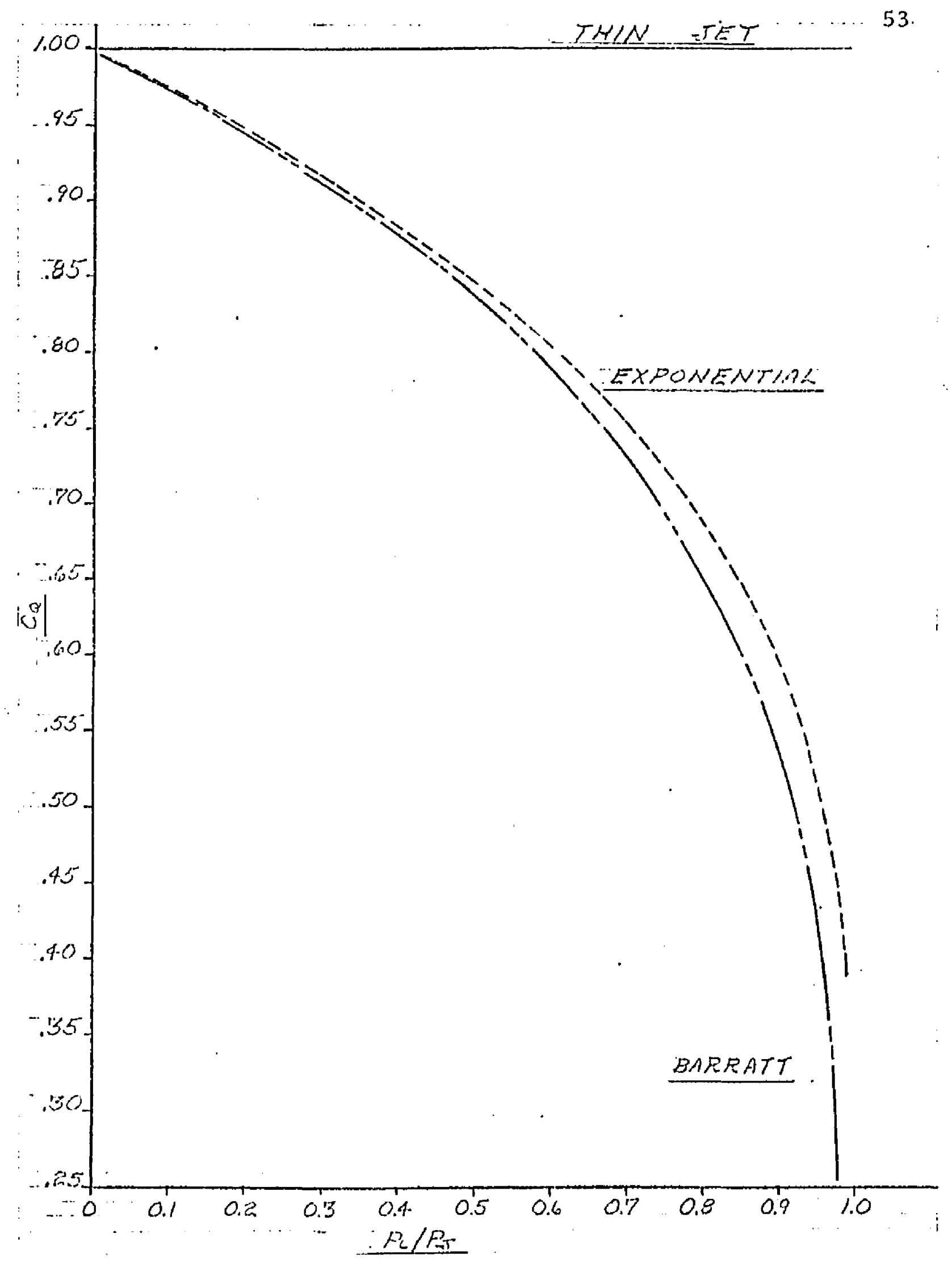

FLOW COEFFICIENT vs $p_{c} / p_{s}$ FIGURE 3-2 


\section{Exponential theory}

$o_{j}=\operatorname{ts} \sqrt{\frac{2 g_{o}}{\rho}\left(p_{j}\right)}\left[\frac{1}{x}\left(1-e^{-x}\right)\right]$

Barratt theory

$\mathrm{Q}_{\mathrm{j}}=\mathrm{ts} \sqrt{\frac{2 \mathrm{~g}_{\dot{\rho}}}{\rho}\left(\mathrm{p}_{\mathrm{j}}\right)}\left[\frac{\sqrt{1-\mathrm{p}_{\mathrm{c}} / \mathrm{p}_{\mathrm{j}}}}{1-\sqrt{1-\mathrm{p}_{\mathrm{c}} / \mathrm{p}_{\mathrm{j}}}} \log \mathrm{g}_{\mathrm{c}}\left(1-\mathrm{p}_{\mathrm{c}} / \mathrm{p}_{\mathrm{j}}\right)^{-\cdots / 2}\right]$

Equations (2-28), (2-39), and (2-56) were constructed so that the flow is dependent upon a standard reference pressure $\left(\mathrm{p}_{\mathrm{j}}\right)$ multiplied by a factor to compensate for the pressure g!radient across the jet thickness. The factor in brackets defines pressure coefficient, $\mathrm{C}_{\mathrm{Q}}$.

The pressure coefficient, $C_{Q}$, is defined from Equations (2-23), (2.39), or (2.56) as follows:

$$
\mathrm{c}_{\mathrm{Q}} \equiv \frac{\mathrm{Q}_{\mathrm{j}}}{\mathrm{ts} \sqrt{\frac{2 \mathrm{~g}_{\mathrm{O}}}{\rho}}\left(\mathrm{P}_{\mathrm{j}}\right)}
$$

Graphs of $C_{O}$ versus $p_{c} / p_{j}$ are shown in Figure 3-2. The expressions for $C_{Q}$ are summarized in Table 3-1.

Using the pressure coefficient, it is possible to write a general flow equation for the total flow from an actual concentrated peripheral jet air suspension system. The relationship is:

$$
\mathrm{a}_{\mathrm{j}}=\mathrm{st} \sqrt{\frac{2 \mathrm{~g}_{\mathrm{O}}}{\rho}\left(\mathrm{p}_{\mathrm{j}}\right)} \mathrm{c}_{\mathrm{Q}} \mathrm{c}_{\mathrm{x}}
$$

where:

$$
\mathrm{C}_{\mathrm{x}}=\text { coefficient of discharge for jet nozzle with } \mathrm{p}_{\mathrm{c}} / \mathrm{p}_{j}=0
$$




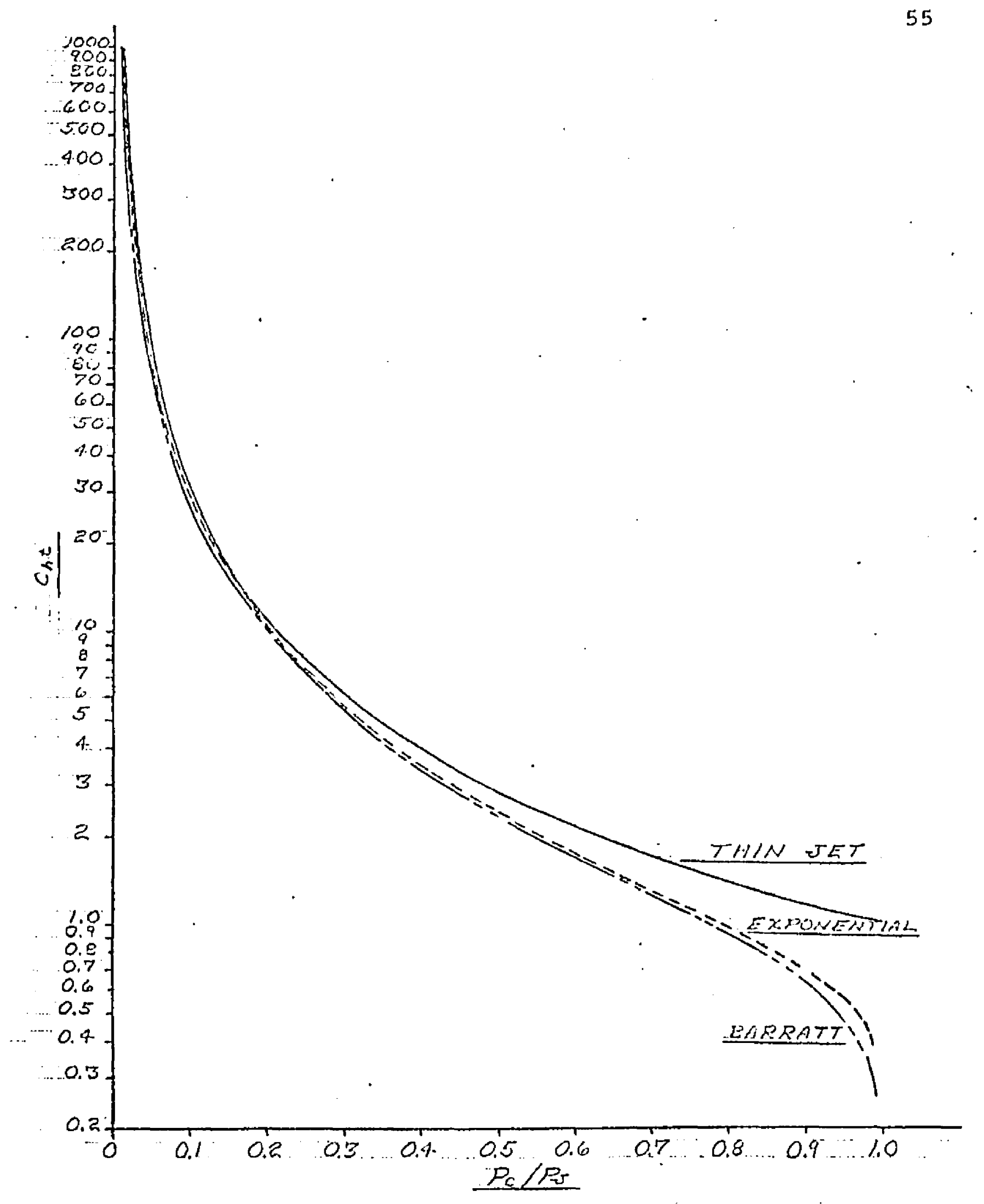

POWER-THICKNESS PARAMETER V $p_{c} / p_{J}$

FIGURE $3-3$ 
$\mathrm{C}_{\mathrm{Q}}=$ pressure coefficient which compensates for pressure gradient across the jet.

\subsection{Power Thicknoss Parameter}

The power-thickness parameter, $\mathrm{C}_{h t}$, is a dimensionless parameter useful in visualizing the effect of trunk plessure on power requirements. This parameter may be developed from the gencral horsopower equation (2-10) and the general flow equation ( $3-4$ ). These equations are:

$$
\begin{aligned}
& h p=\frac{p_{j} Q_{j}}{550} \\
& Q_{j}=S t \sqrt{\frac{2 g_{O}}{\rho}\left(p_{j}\right)} C_{Q} C_{x}
\end{aligned}
$$

Equations (2.10) and (3.4) may be combined to yield:

$$
h p=\left(p_{j}\right)^{3 / 2} s t \sqrt{\frac{2 g_{o}}{\rho}} \frac{C_{Q} C_{X}}{550}
$$

A dimensionless relationship may be developed by rearranging Equation (3-5) and dividing both sides by $\left(p_{c}\right)^{3 / 2}$. The resulting relationship forms the basis for defining the power-thickness parameter, $\mathrm{C}_{\text {ht }}$.

$$
\mathrm{c}_{h t} \equiv \frac{\langle h p)(550)}{\mathrm{st} \sqrt{\frac{2 g_{o}}{\rho}\left(p_{c}\right)^{3 / 2}}}=\left(\frac{\rho_{j}}{\rho_{c}}\right)^{3 / 2} c_{Q} c_{x}
$$

For a given load, cushion area, cushion periphery and jet configuration, the parameter $C_{h t}$ is directly proportional to horsepower. A plot of $\mathrm{p}_{\mathrm{c}} / \mathrm{p}_{\mathrm{j}}$ versus $\mathrm{C}_{\mathrm{ht}}$ (see 


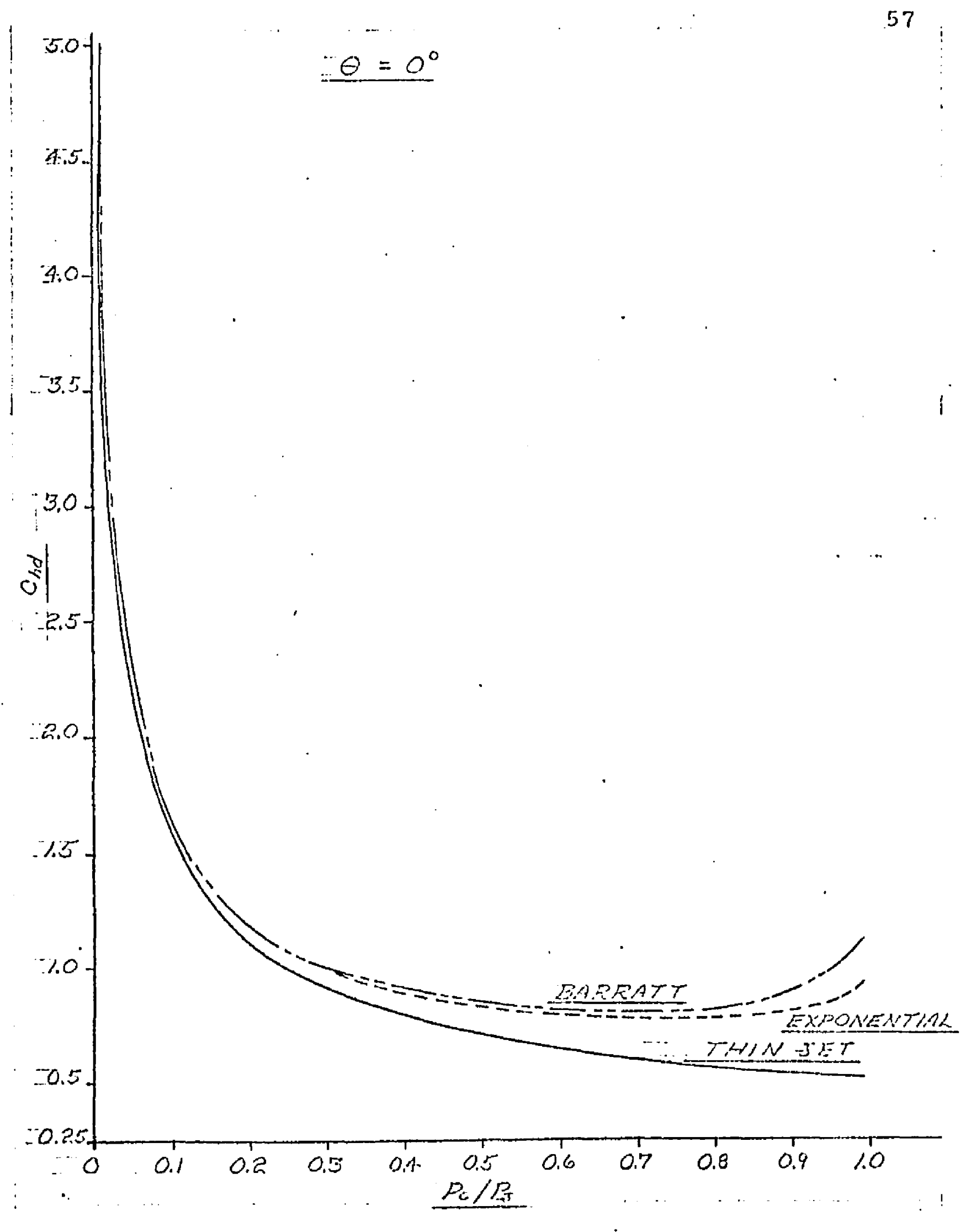

POWER-HEIGHT VS $P_{C} / P_{3}$, SIMPLE JET THEORY FIGURE 3-na 


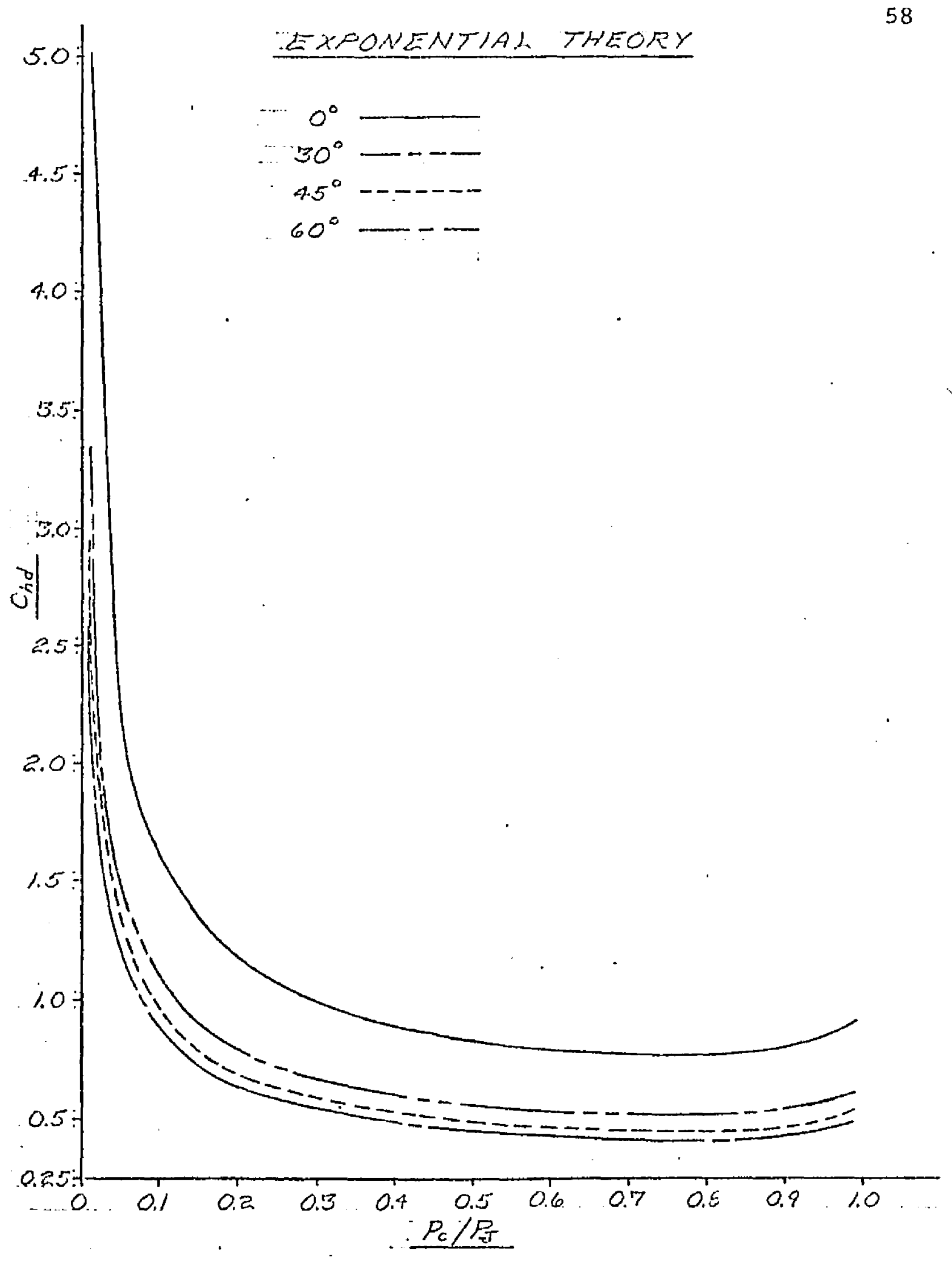

POWER-HEIGHT PARAMETER VS $p_{c} / p_{J}$, EXPONENTIAL THEORY FIGURE 3-4b 


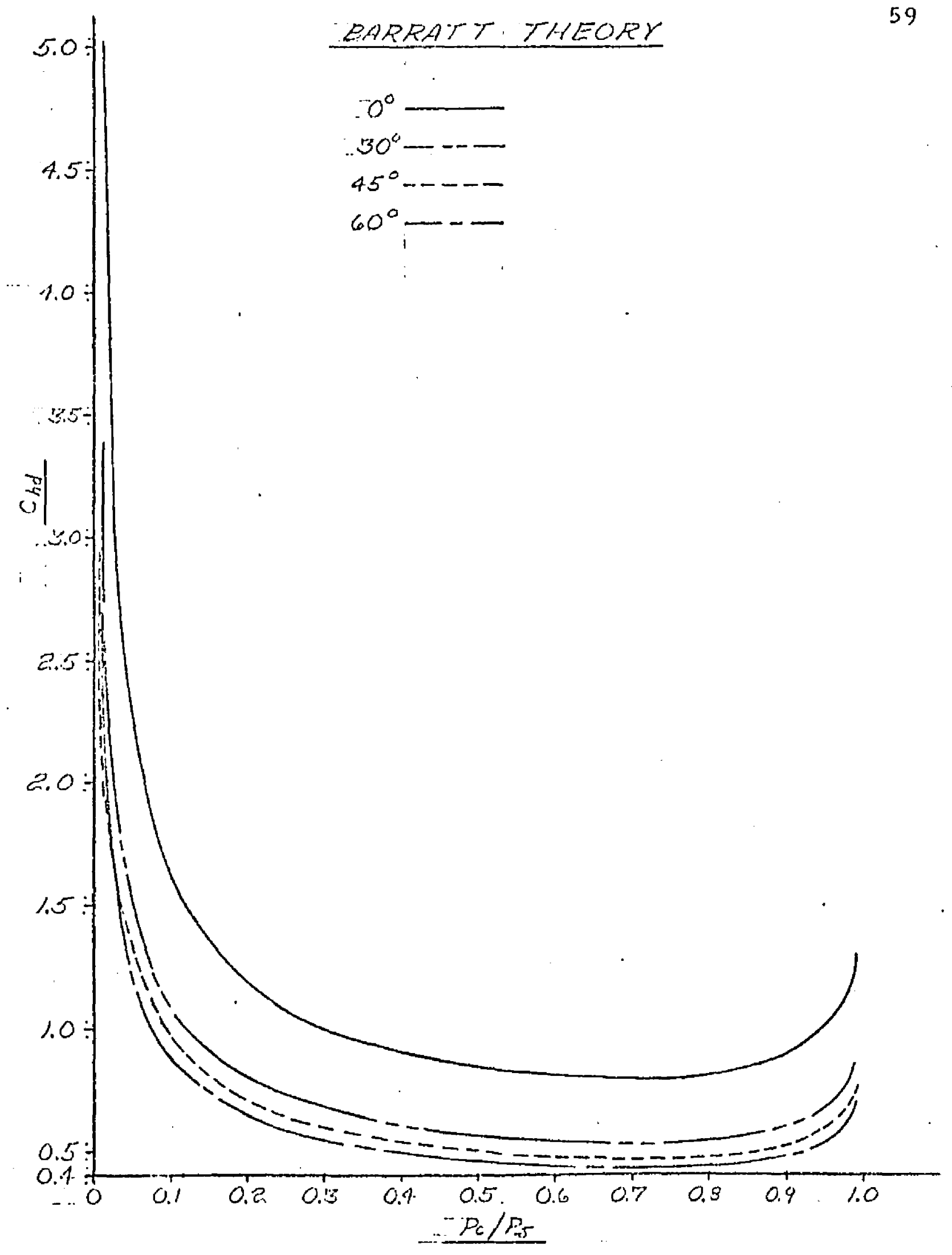

POWER-HEIGHT PARAMETER VS $p_{c} / p_{1}$, BARRETT THEORY FIGURE 3-4c 
Figure 3-3) shows how, other parameters being constant, increases in trunk pressure cause increases in horsepower.

\subsection{Power-Height Parameter}

The power-thickness parameter, developed in Section 3.5, does not include the jet height (d) in the rclationship. In the design of a peripheral jet air cushion system, it is generally desirable to maximize jet height and minimize power. A dimensionless parancter which includes both horsepower and jet height may be developed by multiplying both sides of Equation (3-6) by the ratio ( $t / d$ ). The result is defined as $C_{\text {hid }}$ the power-height parameter.

$$
c_{h d}=\left(\frac{h p}{d}\right)\left(\frac{550}{s}\right) \frac{1}{\sqrt{\frac{2 g_{O}}{\rho}}}\left(\frac{1}{\left(p_{c}\right)^{3 / 2}}\right)=\left(\frac{t}{d}\right)\left(\frac{p_{j}}{p_{c}}\right)^{3 / 2} \quad c_{Q} c_{x}
$$

Equation (3-7) contains horsepower and jet height as a ratio. Since it is desirable to minimize power and maximize jet height, a minimum value of the parameter $\mathrm{C}_{\text {hd }}$ should be selected as a design point.

Graphs of $C_{h d}$ versus $p_{c} / p_{j}$ for $0=0$ and $C_{x}=1.0$ are shown in Figure 3.4(a). The effect of 0 is shown in Figure $3.4(\mathrm{~b}$ and $\mathrm{c})$. It is evident from Figure 3.4 (a) that design points in the range of $\mathrm{p}_{\mathrm{c}} / \mathrm{p}_{\mathrm{j}}=0.4$ to $\mathrm{p}_{\mathrm{c}} / \mathrm{p}_{\mathrm{j}}=0.9$ are desirable from a maximum jet height, minimum power standpoint.

\subsection{Augmentation Ratio}

The augmentation ratio is, in fact, a lift cocfficient for the vehicle. This parameter is defined at least seven different ways in the literature, ${ }^{(8)}$ depending on the choice of the reference force in Equation (3-1). Only one definition will be considered here. The reference force assumed here is the maximum thrust which could be generated if the jet nozzle 
exhaust were discharged vertically downward. This thrust has been designated $F_{\mathrm{j}}$. The expression for the augmentation ratio is:

$$
A_{I}=\frac{\text { pressure support }+ \text { actual jet thrust in vertical direction }}{\text { ideal jet thrust }}
$$

or

$$
A_{l}=\frac{p_{c} A_{c}+F_{j} \cos \theta}{F_{j}}
$$

An expression for $F_{j}$ may be cleveloped by evaluating the total change of momentum in the vertical direction for the air as it flows from the trunk to the atmosphere. If the simple jet theory is assumed, the magnitude of the total momentum of the jet at the exhaust plane is given by Equation (2-26).

$$
\mathrm{J}^{\prime}=2 \mathrm{St} \mathrm{p}_{\mathrm{j}}
$$

The momentum of the gas in the trunk is assumed to be zero. The magnitude of the jet thrust may be written:

$$
F_{j}=2 S t p_{j}
$$

Equation (3-9) may be substituted into Equation (3-8) and the result rearranged to give an expression which relates $A_{1}$ to $p_{c} / p_{j}$. The resulting equation is:

$$
A_{l}=\cos 0+\left(p_{c} / p_{j}\right)\left(\frac{A_{c}}{2 S t}\right)
$$

Equation (3-10) may be further simplified by assuming the cushion is circular in shape. For a circular shaped cushion with a diameter, $D$, 


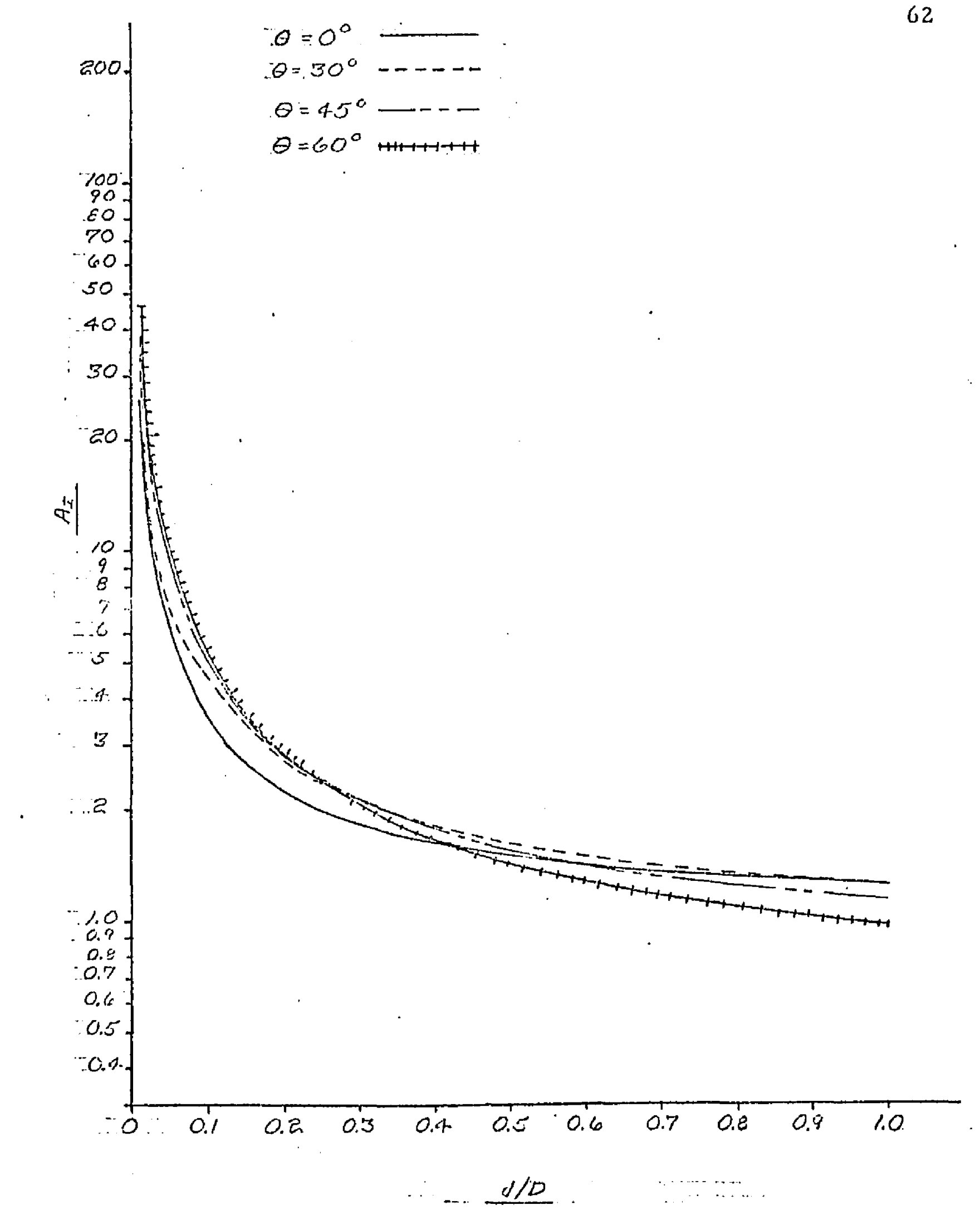

AUGMENTATION VS JET HEIGHT TO CUSHION DIAMETER RATIO FIGURE $3-5$ 


$$
\begin{aligned}
& A_{c}=\frac{\pi D^{2}}{4} \\
& S=\pi D
\end{aligned}
$$

The recovery pressure $\left(\mathrm{p}_{\mathrm{c}} / \mathrm{p}_{\mathrm{j}}\right)$ may be written:

$$
P_{c} / P_{j}=2 \frac{t}{d}(1+\sin 0)
$$

The above three relationships may be substituted into Equation (3-10) to give:

$$
A_{1}=\cos \theta+\frac{1}{4 d / D}(1+\sin \theta)
$$

Equation (3-11) expresses the augmertation ratio in terms of jet angle, cushion diameter and jet height. A circular cushion (plenum) area and the simple jet theory were assumed in developing Equation (3-11).

The influence of $d / D$ on $A_{1}$ for various values of jet angle 0 is shown in Figure 3.5.

\subsection{Summary of Results}

The influence of $\mathrm{p}_{\mathrm{c}} / \mathrm{p}_{j}$ on the nozzle thickness parameter is shown in Figure 3-1. The inverse of the nozzle thickness parameter is directly proportional to jet height. Consequently, Figure 3-1 shows how the jet height varies with $\mathrm{p}_{\mathrm{c}} / \mathrm{p}_{\mathrm{j}}$ for constant values of nozzle thickness $(t)$ and jet angle $(\theta)$. This figure shows that jet height increases with decreasing $\mathrm{p}_{\mathrm{c}} / \mathrm{p}_{\mathrm{j}}$. It may be recognized that a decreasing $\mathrm{p}_{\mathrm{c}} / \mathrm{p}_{\mathrm{j}}$ implies an increasing $\mathrm{p}_{\mathrm{j}}$, if $\mathrm{p}_{\mathrm{c}}$ is held constant. The figure suggests that jet height increases with increasing $\mathrm{P}_{\mathrm{j}}$. This result is intuitively appealing. The three theories shown give similar results for small values of $p_{c} / p_{j}$ but diverge with increasing $\mathrm{p}_{\mathrm{c}} / \mathrm{p}_{\mathrm{j}}$. The Barratt theory has been shown (Reference 41 ) to give the closest agreement with experimental results. The exponential theory is useful because of 
its relative simplicity and its close agreement with the more complicated Barratt theory. The simple jet theory is accurate only at low values of $\mathrm{p}_{\mathrm{c}} / \mathrm{p}_{\mathrm{j}}$ and $X$ (say $\mathrm{p}_{\mathrm{c}} / \mathrm{p}_{\mathrm{j}}<0.4$ and $X<$ $0.2)$. It is usefui in developing simple preliminary relationships and trends.

The influence of $p_{c} / p_{j}$ on the pressure coefficient $\left(C_{Q}\right)$ is shown in Figure 3-2. For the theories presented, this relationship is inclependent of the jet angle, 0 . The figure shows that a high value of $\mathrm{p}_{\mathrm{c}} / \mathrm{p}_{\mathrm{j}}$ is desirable to minimize this coefficient.

The influence of $\mathrm{p}_{\mathrm{c}} / \mathrm{p}_{\mathrm{j}}$ on the power-thickness parameter is shown in Figure 3-3. The parameter, $C_{h t}$ is directly proportional to input power. Figure 3-3 shows that, for constant values of nozzle area and cushion pressure (aircraft weight), high values of $\mathrm{p}_{\mathrm{c}} / \mathrm{p}_{\mathrm{j}}$ (low values of $p_{j}$ ) are desired for minimum power.

The influence of $p_{c} / p_{j}$ on the power-height parameter $\left(C_{h d}\right)$ is shown in Figure 3-4. It is generally desirable to minimize power and maximize jet height. For constant $p_{C}$ (aircraft veight), and fuselage perimeter $(S)$, a minimum $C_{h d}$ would give a maximum jet height and minirnum power $i$,put. Figure $3.4(a)$ shows that both the exponential and the Barratt theory give $C_{\text {hd }}$ curves with mirimum values around $\mathrm{P}_{c} / \mathrm{p}_{\mathrm{j}}=0.7$. Since the curve is flat in the region of $\mathrm{P}_{\mathrm{c}} / \mathrm{P}_{\mathrm{j}}=0.4$ to $\mathrm{P}_{\mathrm{c}} / \mathrm{P}_{\mathrm{j}}=0.9$ a considerable latitude exists in sclecting an optimum $\mathrm{p}_{\mathrm{c}} / \mathrm{p}_{\mathrm{j}}$.

The influence of 0 on the power-jet height parameter is shown in Figure 3.4(b and c). The curves show that a high value of 0 is desirable. However, if $\theta$ becomes too large, the flow will attach to the underside of the aircraft and the momentum seal will be lost. A value of $\theta=60^{\circ}$ is generally considered as the maximum practical.

The effect of the jet height to cushion diameter ratio on augmentation ratio for a circular cushion is shown in Figure 3-5. The figure shows that it is desirable to have small values of $d / D$ for maximum augmentation. Large values of augmentation are desirable to minimize power. The value of jet height (d) is generally determined by the roughness of the terrain on which the vehicle is designed to operate. Consequently, $d$ is largely independent of vehicle size. For maximum augmentation it is desirable to make the cushion diameter as 
large as possible without violating structural weight and dynamic constraints.

In summary, Figure 3-3 shows that power decreases with increasing $\mathrm{p}_{\mathrm{c}} / \mathrm{p}_{\mathrm{j}}$ if jet height is allowed to decrease. However, for a sjecified value of jet height it is desirable to select a value of $\mathrm{P}_{\mathrm{c}} / \mathrm{p}_{\mathrm{j}}$ in the range of 0.4 to 0.7 . Figures $3.4(\mathrm{~b}$ and $\mathrm{c}$ ) show that it is desirable to employ a jet angle 0 of at least $30^{\circ}$. Larger angles, up to $60^{\circ}$, give slight additional benefits in minimizing the power-height parameter. Finally, Figure 3.5 shows that it is desirable to make the vehicle diameter large and the jet clearance small for maximum augmentation. 
TABI: 3-1

Expressions for $F_{0} b_{j}$ and $C_{Q}$ for Momentum Flow Theories

\begin{tabular}{|c|c|c|}
\hline PARAMETER & $p_{c} / F_{j}$ & $c_{Q}$ \\
\hline SIMPLE JET & $2 \mathrm{x}$ & 1.0 \\
\hline EXPONENTIAL & $1-e^{-2 x}$ & $\frac{1}{x}\left(1-e^{-x}\right)$ \\
\hline BARRAIT & $2 x\left(\sqrt{x^{2}+1}-x\right)$ & {$\left[\frac{\sqrt{1-p_{\mathrm{c}} / p_{j}}}{i-\sqrt{1-p_{c} / p_{j}}} \log _{e}\left(1-p_{c} / p_{j}\right)^{-1 / 2}\right.$} \\
\hline
\end{tabular}




\section{PREDICTION OF THE SHAPE OF A TWO DIMENSIONAL AIR CUSHION TRUNK}

\section{1 $\quad$ Approach}

Accurate predictions of the cross-sectional shape and area of the air cushion trunk are necessary in determining the flow rate, jot height, stiffness and dynamic response of the system. It is desired to predict the trunk shape when it is subjected to two types of loading.

The first type occurs when the aircraft is being supported totally by the air cushion. In this case, the trunk transmits none of the load directly to the ground. The trunk shape associated with this type of loading is illustrated by Figure 4-1. This case is called the Free Trunk Shape. It is developed in cletail in Section 4.4.

The second type of loading occurs cluring dynamic loading of the air cushion. In this case, a portion of the trunk may be flattened against the ground and transmits loads to the ground through a thin layer of air. The trunk shape associated with this type of loading is illustrated in Figure 4-2. This case is called the Loaded Trunk Shape. It is developed in Section 4.5. Computer programs which predict these two shapes for an inelastic trunk material are presented in Appendices 1 and 11 respectively. Appendix III contains a computer program for predicting the Free Trunk Shape including the effects of trunk material which have non-linear elasticity.

\subsection{Background}

The configuration and loading of the trunk of the Air Cushion Landing System is considerably different from the trunk on Air Cushion Vehicles. Consequently, the literature associated with air cushion vehicle trunks is of little assistance in predicting the ACLS shape.

Esger and Morgan ${ }^{(44)}$ conducted an analysis of the energy absorptive characteristics of gas bags of various shapes and at various rates of gas bleed. The study 


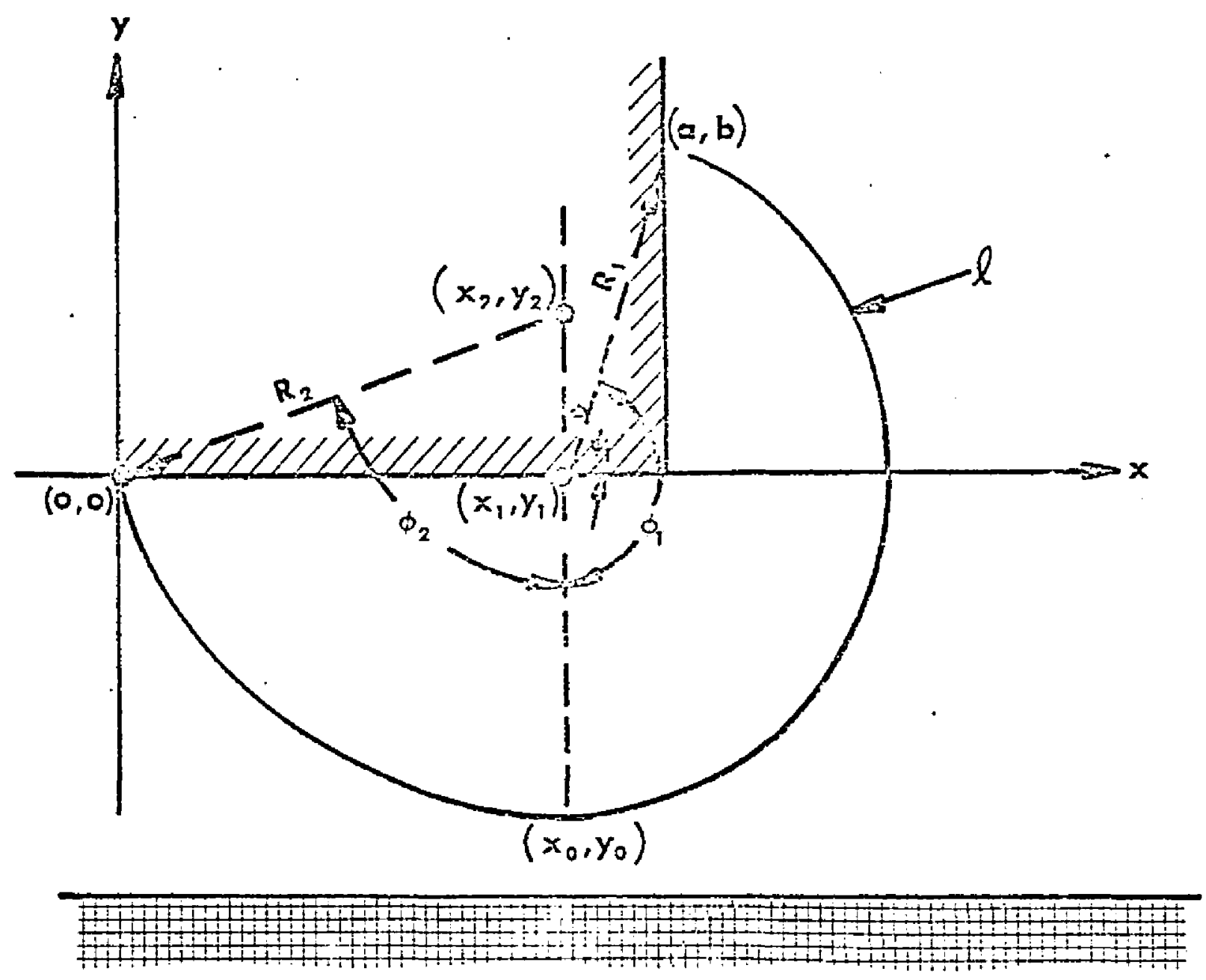

FREE TRUNR SHAPE

FIGURE 4-? 


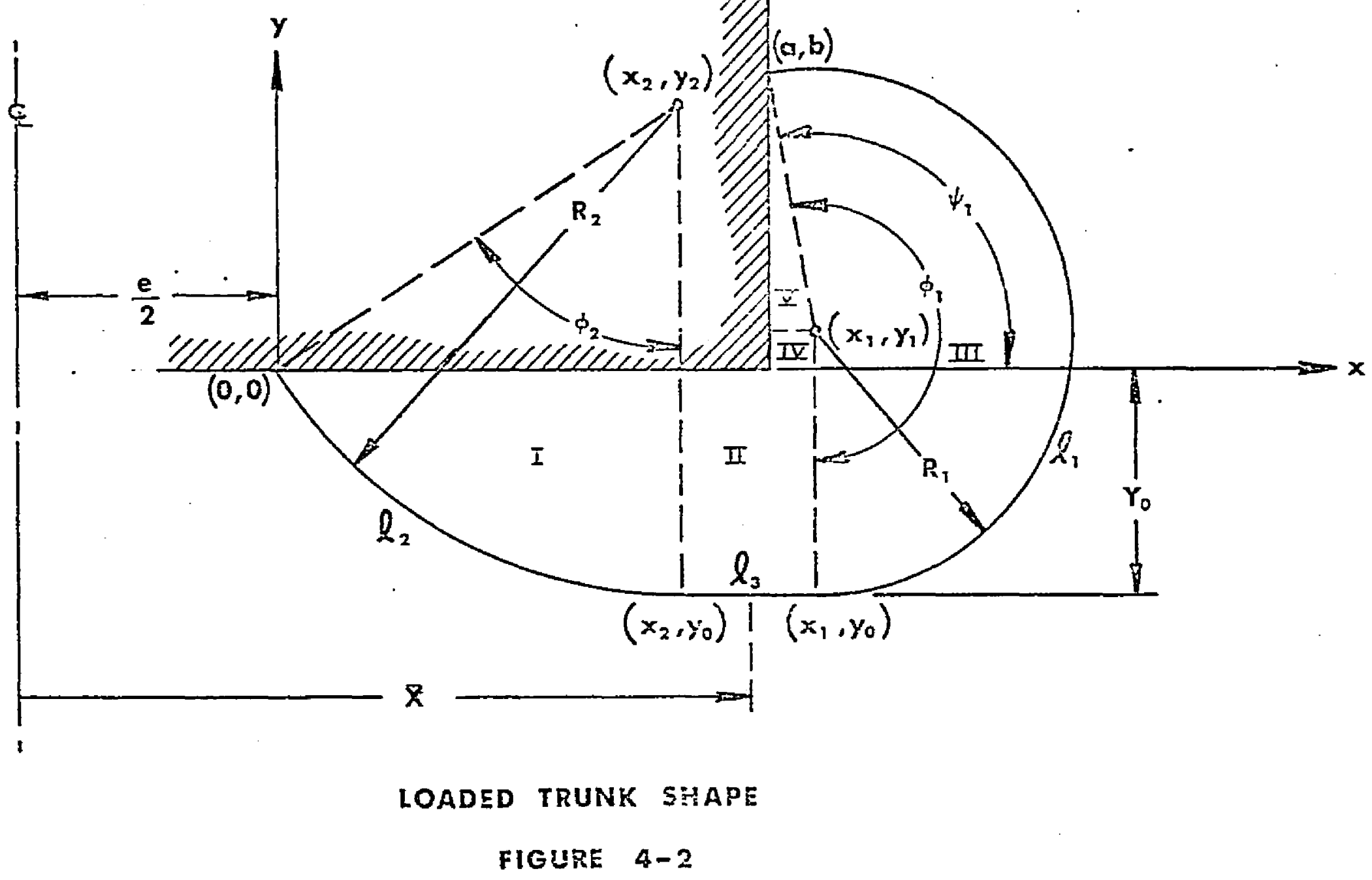


included cylindrical shaped bags impacted on their sides. This case approaches the Loaded Trunk Shape problem. These authors found that the deflected cross-sectional shape approximated two circular arcs tangent to the ground surface and connected by a straight line at the ground contact. A similar condition is shown in Figure 4-2.

In the sections to follow, numerical solutions to predict the shape of the trunk under both free and loaded conditions are presented. Digital computer programs which evaluate the trunk shape for these conditions are presented in Appendix I and Appendix II.

The relationships which are common to both the free and the loaded trunk shape are presented in Section 4.3.

\subsection{Development of Common Relationships}

\subsubsection{Approach}

In this section, the variables associated with the trunk shape are listed, the laws which will be applied are stated, and the relationships which are common to both problems are developed.

The variables for this problem are illustrated in Figures $4-1$ and $4-2$. They may be grouped as folluws:

Independent Design Variables

a(a) $x$ coordinate of (horizontal distance between) trunk attachment points, $\mathrm{ft}$.

b(b) $\quad y$ coordinate of (vertical distance between) trunk attachment points, $\mathrm{ft}$.

e distance between lower trunk attachment points, $\mathrm{ft}$ (see Figure 4-8). 
$\ell \quad$ cross-sectional length of trunk material, $\mathrm{ft}$.

$\ell_{0} \quad$ cross-sectional length of the trunk material at the design point, ft (see Figure 4-14).

$E_{t}$ the unit elongation per pound of tension per foot-length in the axial direction for the trunk material, $1 \mathrm{~b} / \mathrm{ft}$ (see Figure 4-14).

Independent Operating Variables for Free Trunk Shape

$P_{c}\left(P_{C}\right) \quad=$ cushion pressure, psfg (psf).

$p_{j}\left(P_{j}\right) \quad=$ trunk pressure, $p s f g(p s f)$

For the Loaded Trunk Shape, one additional independent variable is:

$Y_{0} \quad=$ vertical distance between the aircraft hard structure and bottom of the trunk (ft).

Dependent Variables

$\ell_{1} \quad$ length of trunk segment inscribed by angle $\phi_{1}, \mathrm{ft}$.

$\ell_{2} \quad$ length of trunk segment inscribed by angle $\phi_{2}, \mathrm{ft}$.

$\ell_{3} \quad$ length of trunk segment flattened against the ground, $\mathrm{ft}$.

$R_{1} \quad$ radius of curvature for segment $\ell_{1}, f$ t.

$R_{2}$ radius of curvature for segment $\ell_{2}, f$ t. 
$T_{t} \quad$ tension in trunk material, pounds in tangential direction per foot-length in the axial direction, $\mathrm{lb} / \mathrm{ft}$.

$\bar{x} \quad$ distance from aircraft center of gravity to center of pressure of the trunk footprint, $\mathrm{ft}$.

$x_{i} \quad x$ coordinate of $i^{\text {th }}$ point, $f t$.

$y_{i} \quad y$ coordinate of $i^{\text {th }}$ point, $f t$.

$\phi_{1} \quad$ central angle formed by trunk segment $\ell_{1}$, radians.

$\phi_{2} \quad$ central angle formed by trunk segment $\ell_{2}$, radians.

The laws to be upplied to this problem are:

(1) Force equilibrium applied to the trunk

(2) Load-elongation of the trunk

(3) Geometric compatibility of the trunk shape

The first two laws hold for both trunk shapes. The difference in the two problems lies in the geometric compatibility assumptions. Consequently, the first two relationships will be developed in Sections 4.3.2 and 4.3.3 to follow.

\subsubsection{Force Equilibrium}

Consider an elastic material of length $\ell$ attached to the structure at points $\{a, b\rangle$ and $(0,0)$ as shown in Figures $4-1$ or $4-2$. The trunk is subjected to an internal pressure $P_{j}$, to 
a cushion pressure $P_{c}$ and to atmospheric pressure $P_{a}$. The following assumptions are made:

4.3.2.1 The trunk behaves as a membrane. Thus it forms a segment of a circle when subjected to internal pressure loading.

4.3.2.2 Reactions from the nozzles are negligible.

4.3.2.3. The tension in the trunk is constant in the Sections $\ell_{1}$ and $\ell_{2}$.

Based upon the assumptions, a free body diagram of the loading on the two sections of the trunk is shown in Figure 4-3(a). The tension at any point in the trunk is calculated by a force balance (as shown in Figure 4-3(b) and found to be:

$$
\left(P_{j}-P\right) 2 R \sin \frac{\phi}{2}=2 T_{t} \sin \frac{\phi}{2}
$$

Applying this force balance to the two trunk sections and simplifying gives:

$$
T_{t}=p_{j} R_{1}
$$

and

$$
T_{t}=\left(p_{j}-p_{c}\right) R_{2}
$$

\subsubsection{Load-Elongation of the Trunk}

The length of the trunk material is determined from the tension-elongation characteristics of the material. For a purely elastic material, the tension-elongation relationship is:

$$
\ell=\ell_{0}+\frac{T_{t}}{E_{t}} \ell_{0}
$$




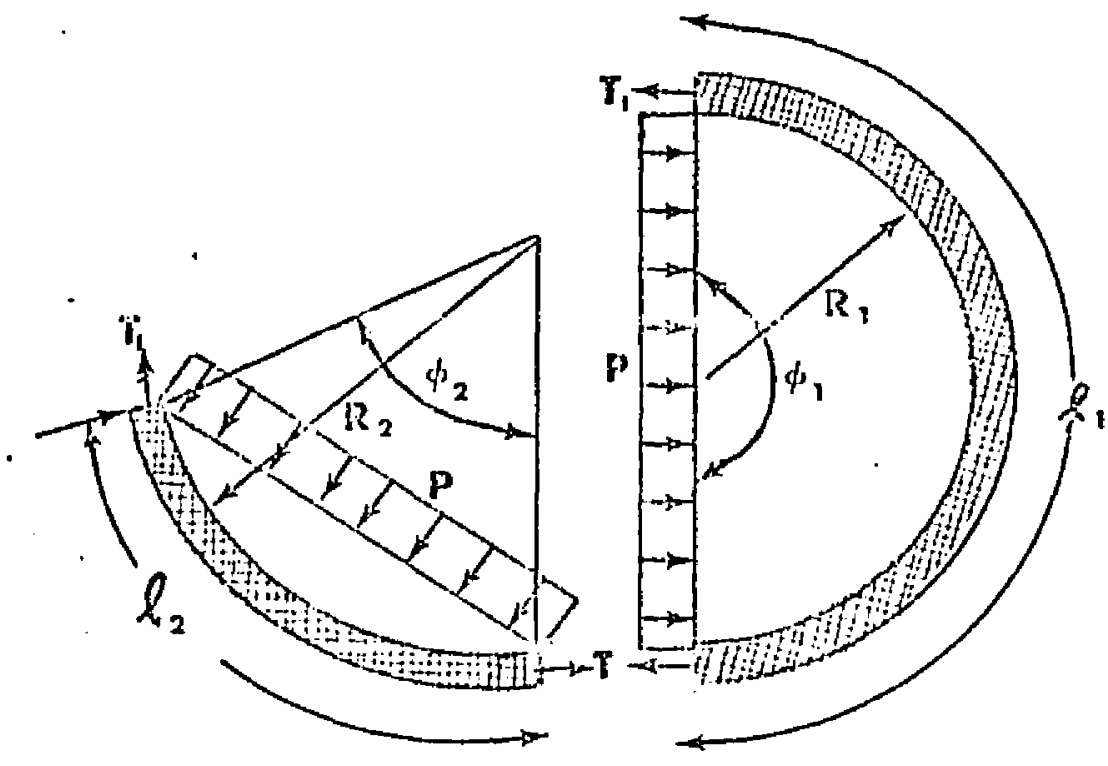

(a) DIAGRAM OF PRESSURE-TENSION EQUILIEIRIUM

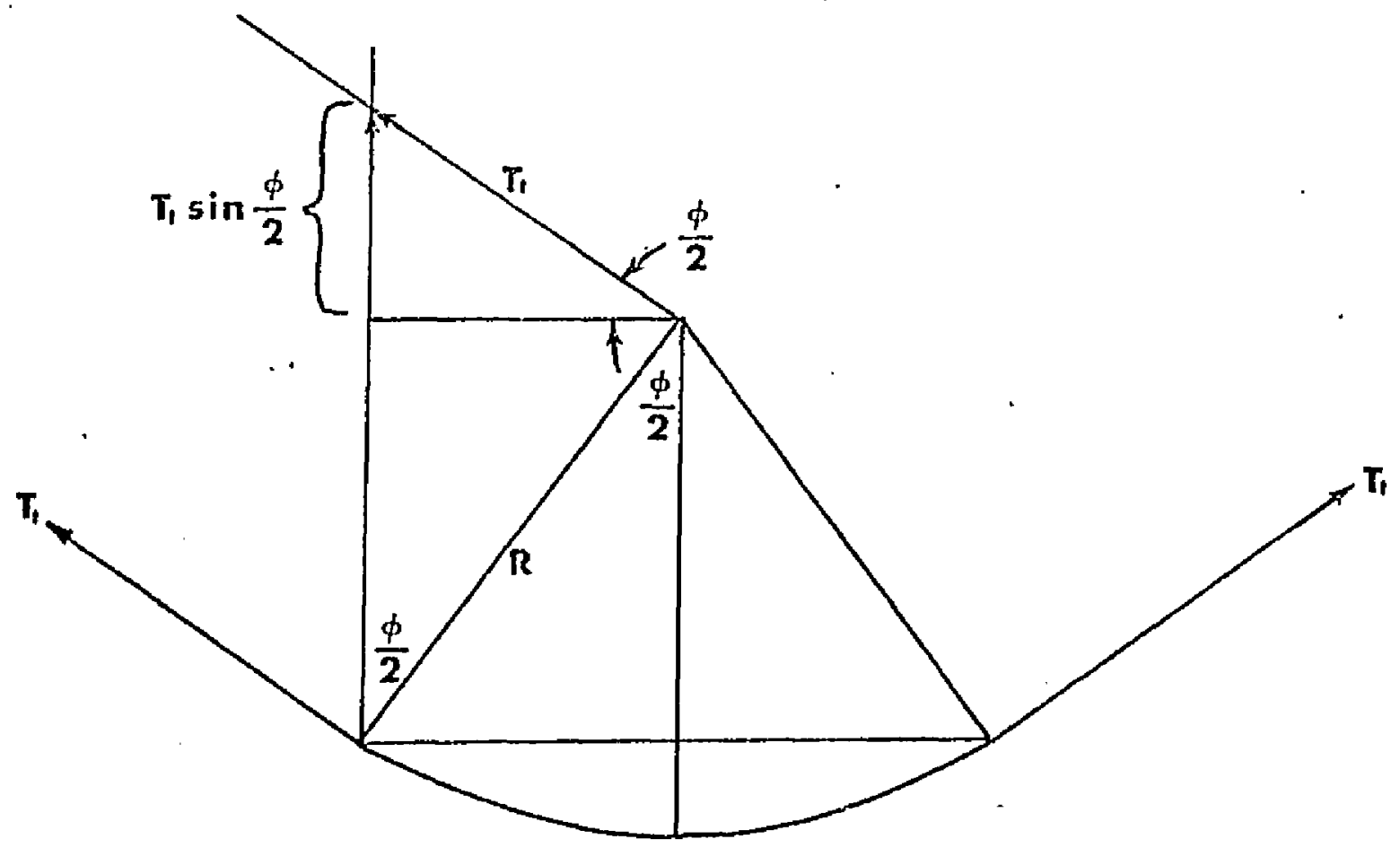

(b) DIAGRAM OF TENSION COMPONENT FREE BODY DIAGRAM OF TRUNK LOADING FIGURE $4-3$ 
In general, the elasticity of trunk materials will be non-linear. Consequently, a more complicated relationship than Equation (4-3) must be used. A typical tension-elongation curve for a trunk material is shown in Figure 4-14.

\subsubsection{Geometric Compatibility}

The geometric compatibility conditions of this free trunk slape problem differ from the loaded trunk shape problem. The differences are shown in Figures 4-1 and 4-2, respectively. Separate development of the geometric compatibility conditions will be presented in Sections 4.4 and 4.5.

\subsection{Free Trunk Shape}

\subsubsection{Assumptions}

A cross section of the free trunk shape is shown in Figure 4-1. In addition to the assumptions listed in Jection 4.3.2 the following restrictions are imposed:

4.4.1.1 The pressure change from $P_{c}$ to $P_{a}$ occurs over a short clistance in the vicinity of point $\left(x_{0}, y_{0}\right)$.

4.4.1.2 The trunk is assumed to be tangent to the ground at point $\left(x_{0}, y_{0}\right)$. No flattening of the trunk around point $\left(x_{0}, y_{0}\right)$ is allowed. This assumption requires that the centers of curvature for radii $R_{1}$ and $R_{2}$ have the same $x$ coordinate.

\subsubsection{Geometric Compatibility (Free Trunk Shape)}

The assumed trunk geometry is shown in Figure 4-1. 
In order for the trunk segments formed by $\ell_{1}$ and $\ell_{2}$ to both be tangent to the ground at $\left(x_{0}, v_{0}\right)$ the centers of curvature must have the same $x$ coordinate. Thus

$$
\begin{aligned}
& x_{1}=x_{0} \\
& x_{2}=x_{0} .
\end{aligned}
$$

The distance between $(0,0)$ and $\left(x_{2}, y_{2}\right)$ is $R_{2}$

$$
\left(x_{2}-o\right)^{2}+\left(y_{2}-o\right)^{2}=R_{2}^{2}
$$

The distance between $\left(x_{1} y_{1}\right)$ and $(a, b)$ is $R_{1}$

$$
\left(x_{1}-a\right)^{2}+\left(y_{1}-b\right)^{2}=R_{1}^{2}
$$

The distance between $\left(x_{0}, y_{0}\right)$ and $\left(x_{2}, y_{2}\right)$ is $R_{2}$. Since $x_{0}=x_{2}$ the distance is simply the $y$ distance:

$$
v_{2}-y_{0}=R_{2}
$$

Similarly, the distance between $\left(x_{0}, y_{0}\right)$ and $\left(x_{1}, y_{1}\right)$ is $R_{1}$.

$$
V_{1}-y_{0}=R_{1}
$$

The arc formed by $\ell_{2}$ is defined by $\phi_{2}$. The angle $\phi_{2}$ may be written in trigometric terms as:

$$
\phi_{2}=\arctan \frac{x_{2}-0}{y_{2}-0} ; \quad 0 \leqslant \phi_{2} \leqslant \pi \text { radians }
$$


The arc formed by $\ell_{1}$ is defined by $\phi_{1}$. The angle $\phi_{1}$ may be written in terms of the angle $\psi_{\uparrow}$ which is defined in Figure 4-1.

$$
\phi_{1}=\psi_{1}+\frac{\pi}{2} \quad \text { o } \leqslant \phi_{1} \leqslant 2 \pi
$$

The angle $\psi_{4}$ may be written in trigometric terms as:

$$
\psi_{1}=\arctan \frac{b-y_{1}}{a-x_{0}} \quad \frac{-\pi}{2} \leqslant \psi_{1} \leqslant 3 \frac{\pi}{2}
$$

The total length of the trunk is equal to the sum of the two segments:

$$
\ell=R_{1} \phi_{1}+R_{2} \phi_{2}
$$

\subsubsection{Solution of Equations}

In Equations (4-1) through (4-13) the following variables are known:

$a, b, p_{c} / p_{j}, l_{o}, E_{t}, P_{j} \cdot$

The following variables are unknown:

$$
T_{t}, R_{1}, R_{2}, l, x_{0}, x_{1}, x_{2}, y_{0}, y_{1}, y_{2}, \phi_{1}, \phi_{2}, \psi_{1}
$$

In principle, the thirteen equations can be solved simultaneously to predict the unique trunk shape for the given kno:vn quantities.

Equations (4-1) and (4-2) may be combined to solve for $R_{2}$ :

$$
R_{2}=R_{1} / 1-\left(p_{c} / p_{j}\right)
$$


Equations (4-4) through (4-9) may be combined to solve for $y_{0}$. Combining Equations (4-4), (4.6) and (4-8) gives:

$$
x_{0}^{2}+\left(y_{0}+R_{2}\right)^{2}=R_{2}^{2}
$$

or

$$
x_{0}^{2}=-y_{0}^{2}-2 y_{0} R_{2}
$$

Combining Equations (4-5), (4-7), and (4-9) gives:

$$
\left(x_{0}-a\right)^{2}+\left(y_{0}+R_{1}-b\right)^{2}=R_{1}^{2}
$$

or

$$
x_{0}^{2}=2 a x_{0}-a^{2}-y_{o}^{2}-b^{2}+2 R_{1} b+2 y_{o} b-2 R_{1} y_{0}
$$

Equating $(4-15)$ and $(4-16)$ to eliminate $x_{0}{ }^{2}$ yields:

$$
-y_{o}^{2}-2 y_{o} R_{2}=2 a x_{o}-a^{2}-y_{o}^{2}-b^{2}+2 R_{1} b+2 y_{o} b-2 R_{1} y_{o}
$$

or

$$
x_{0}=\frac{y_{0}}{a}\left(R_{1}-b-R_{2}\right)+\frac{a}{2}+\frac{b^{2}}{2 a}-R_{1} \frac{b}{a}
$$

Let

$$
\begin{aligned}
& c_{1}=\frac{R_{1}-b-R_{2}}{a} \\
& c_{2}=\frac{a}{2}+\frac{b^{2}}{2 a}-R_{1} \frac{b}{a}
\end{aligned}
$$


Then Equation (4-17) becomes:

$$
x_{0}=c_{1} y_{0}+c_{2}
$$

Combining Equations (4-20) and (4-15) yields:

$$
-y_{0}^{2}-2 \dot{y_{0}} R_{2}=\left(C_{1} y_{0}+C_{2}\right)^{2}
$$

or

$$
\left(C_{1}^{2}+1\right) y_{o}^{2}+2\left(R_{2}+C_{1} C_{2}\right) y_{o}+C_{2}^{2}=0
$$

Applying the quadratic formula to Equation (4-21)

$$
Y_{0}=\frac{-2\left(R_{2}+C_{1} C_{2}\right) \pm \sqrt{\left(2 R_{2}+2 C_{1} C_{2}\right)^{2}-4\left(C_{1}^{2}+1\right) C_{2}^{2}}}{2\left(C_{1}^{2}+1\right)}
$$

The choice of positive or negative square root is dependent on the quantities $a, b$, and $\ell$. A physical representation of the two solutions is shown in Figure 4-4. The figure illustrates that for given values of $a$ and $b$ the negative square root requires a larger value of $\ell$ than the positive square root.

In order to devclop criteria for sclecting the sign of the square root, consider the case where $p_{c}=0$. For this case, the trunk takes the shape of the arc of a circle of radius $R_{1}$. In order for the circle to pass through $(0,0)$ and $(a, b)$ the radius $R_{1}$ must equal at least half the distance between the two points. The minimum value for $R_{1}$ would be $1 / 2 \sqrt{a^{2}+b^{2}}$. The value of $\ell$ associated with the minimum value of $R_{\uparrow}$ is $\pi / 2 \sqrt{a^{2}+b^{2}}$. Smaller values of $\ell$ would require larger values of $R_{1}$ but smaller values of $Y_{0}$. Consequently, the positive square root gives the desired solution for this case. Larger values of $\ell$ would require larger values of $R_{1}$ and larger values of $Y_{0}$. Consequently, the negative square root would give the desired solution for this case. 

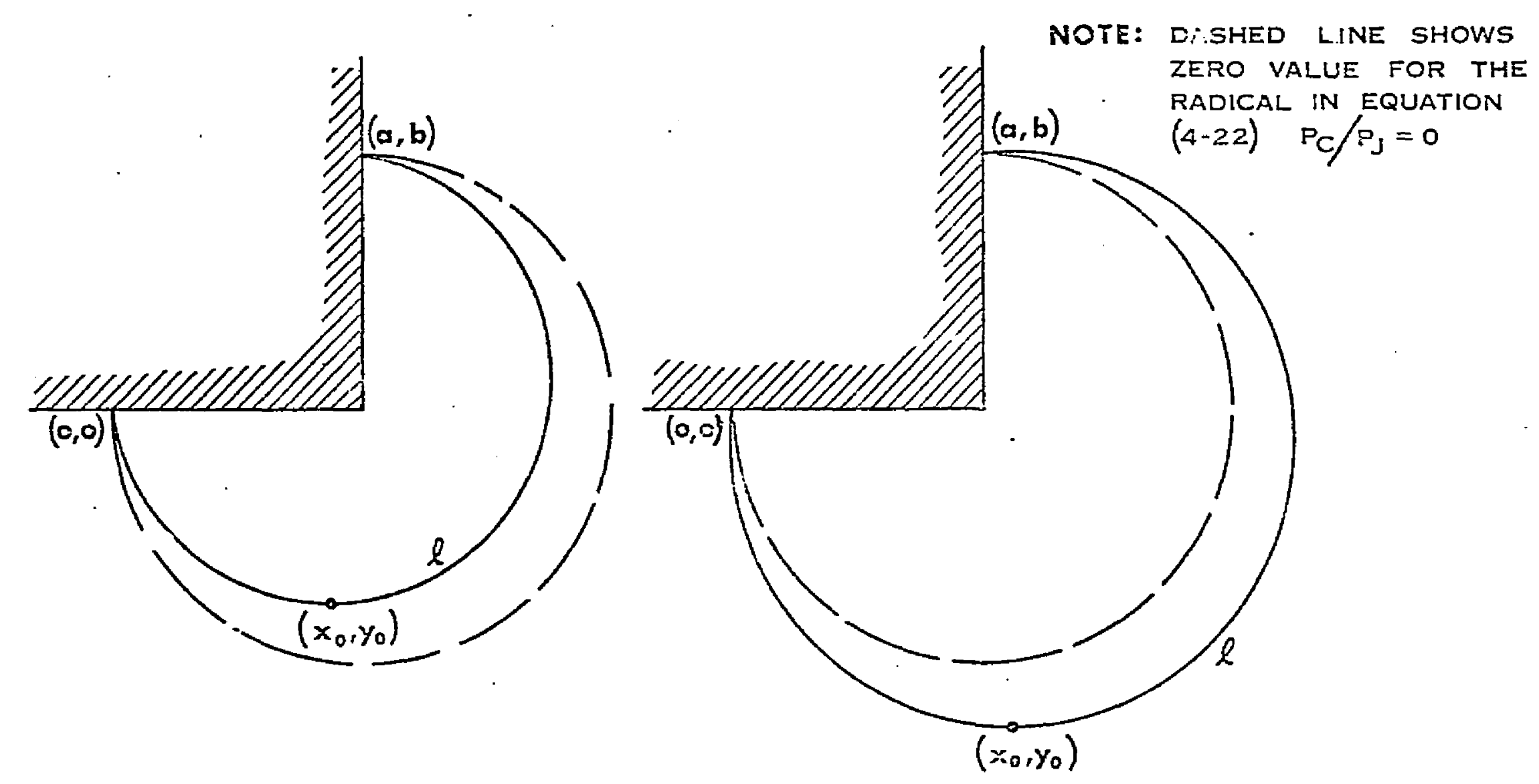

(a) POSITIVE SQUARE' ROOT

(b) NEGATIVE SQUARE ROOT

PHYSICAL INTERPRETATION OF POSITIVE \& NEGATIVE SQUARE ROOT 
When $p_{c} / p_{j}=0$, the criteria for the sign on the square root is as follows.

Take positive root when

$\ell \leqslant \frac{\pi}{2} \sqrt{a^{2}+b^{2}}$

Take negative root when

$\ell>\frac{\pi}{2} \sqrt{a^{2}+b^{2}}$

The problem may now be solved by an iterative process as follows.

The following information is given:

$a, b, \ell_{\alpha}, p_{c} / p_{j}, p_{j}, E_{t}$

The itcrative procedure is as follows:

(1) $R_{1}$ must be assumed for a trial solution. A trial guess is

$$
R_{1}=1 / 2 \sqrt{a^{2}+b^{2}}
$$

12) From Equation (4-1) compute $T_{t}$

$$
T_{t}=p_{j} R_{i}
$$

(3) From Equation (4-3) compute $\&$

$$
\ell=l_{0}+\frac{T_{t}}{E_{t}} \ell_{0}
$$


(4) Calculate the other variables as follows:

$$
\begin{aligned}
& R_{2}=R_{1} / 1-\left(p_{c} / p_{j}\right) \\
& c_{1}=\frac{R_{1}-b-R_{2}}{a} \\
& c_{2}=\frac{a}{2}+\frac{b^{2}}{2 a}-R_{1} \frac{b}{a}
\end{aligned}
$$

$$
y_{0}=\frac{-2\left(R_{2}+C_{1} C_{2}\right) \pm \sqrt{\left(2 R_{2}+2 C_{1} C_{2}\right)^{2}-4\left(C_{1}{ }^{2}+1\right) C_{2}{ }^{2}}}{2\left(C_{1}^{2}+1\right)}
$$

$$
x_{0}=C_{1} y_{0}+C_{2}
$$

$$
v_{1}=y_{0}+R_{1}
$$

$$
v_{2}=v_{o}+R_{2}
$$

$$
\phi_{2}=\arctan \frac{\mathrm{x}_{2}}{\mathrm{Y}_{2}} \quad 0 \leqslant \phi_{2} \leqslant \pi \text { radians }
$$

$$
\psi_{1}=\arctan \frac{b-y_{1}}{a-x_{0}} \quad-\frac{\pi}{2} \leqslant \psi_{1} \leqslant 3 \frac{\pi}{2} \text { radians }
$$

$$
\phi_{1}=\psi_{1}+\pi / 2
$$

$$
\bar{\ell}=R_{1} \phi_{1}+R_{2} \phi_{2}
$$

where $\bar{l}$ is a trial value of $\ell$. 
(5) Check to see if $\bar{l}$ from Equation (4-13) agrees with \& from Equation (4-3). If not, iterate the process. A new guess for $R_{1}$ may be found using Newton's method, Mueller's mothod(45) or othes numerical techniques.

(6) Continue the process until the desired accuracy is obtained in the $\bar{\ell}$ computed from Equation (4-13) and the $\&$ computed from Equation $(4-3)$.

\subsection{Loaded Trunk Shape}

\subsubsection{Assumptions}

The assumed shape of the trunk under an imposed $P_{C^{\prime}} P_{j}$ and $Y_{0}$ is shown in Figure 4-2.

In addition to the assumptions listed in Section 4.3 .2 the following restrictions are added:

4.5.1.1 The pressure on both sides of segment $\ell_{3}$ is equal to $p_{j}$, and $\ell_{3}$ is a straight line.

4.5.1.2 The pressure change from $p_{j}$ to $p_{c}$ and $p_{j}$ to $p_{a}$ occurs instantaneously at points $\left(x_{2}, y_{0} !\right.$ and $\left(x_{1}, v_{0}\right)$ respectively.

4.5.1.3 The trunk is assumed to be tangent to the ground at points $\left(x_{1}, y_{0}\right)$ and $\left(x_{2}, y_{0}\right)$ 


\subsubsection{Geometric Compatibility (Loaded Shape)}

Referring to Figure 4.2, the algebraic relationships for the assumed geometry may be developed as a consequence of Assumption 4.5.1.3:

The distance between $(0,0)$ and $\left(x_{2}, v_{2}\right)$ is $R_{2}$.

$$
\left(x_{2}-o\right)^{2}+\left(y_{2}-o\right)^{2}=R_{2}^{2}
$$

The distance between $(a, b)$ and $\left(x_{1}, v_{1}\right)$ is $R_{1}$.

$$
\left(x_{1}-a\right)^{2}+\left(y_{1}-b\right)^{2}=R_{1}^{2}
$$

The distance between $\left(x_{2}, y_{2}\right)$ and $\left(x_{2}, v_{0}\right)$ is $R_{2}$.

$$
y_{2}-y_{0}=R_{2}
$$

The distance between $\left(x_{1}, y_{1}\right)$ and $\left(x_{1}, y_{0}\right)$ is $R_{1}$.

$$
y_{1}-y_{0}=R_{1}
$$

The distance between $\left(x_{1}, y_{0}\right)$ and $\left(x_{2}, y_{0}\right)$ is $l_{3}$.

$$
x_{1}-x_{2}=\ell_{3}
$$

The arc formed by segment $l_{2}$ is defined by $\phi_{2}$.

The angle $\phi_{2}$ may be written in trigometric terms as:

$$
\phi_{2}=\arctan \frac{x_{2}}{y_{2}} \quad 0 \leqslant \phi_{1} \leqslant \pi
$$


The arc formed by segment $C_{1}$ is defined by $\phi_{1}$. The angle $\phi_{1}$ may be written in terms of the angle $\psi_{1}$.

$$
\phi_{1}=\psi_{1}+\frac{\pi}{2} \quad 0 \leqslant \phi_{1} \leqslant 2 \pi
$$

The angle $\psi_{j}$ may be written in trigonometric terms as:

$$
\psi_{1}=\arctan \frac{b-y_{1}}{a-x_{1}} . \quad-\frac{\pi}{2} \leqslant \psi_{1} \leqslant 3 / 2 \pi
$$

The total length of the trunk must equal the sum of the length of the segments:

$$
\bar{\ell}=\ell_{1}+\ell_{2}+\ell_{3}
$$

where

$$
\begin{aligned}
& \ell_{1}=R_{1} \phi_{1} \\
& \ell_{2}=R_{2} \phi_{2}
\end{aligned}
$$

$\bar{\ell}$ is a trial value of $\ell$.

Using the coordinate system shown in Figure 4-2, we note that:

$$
Y_{0}=-Y_{0}
$$

\subsubsection{Solution of Equations}

In Equations (4-1), (4-2), (4-3), and (4-23) through (4-35), the following variables are known:

$$
a, b, p_{c} / p_{j}, \ell_{0}, E_{t}, p_{j}, Y_{o}
$$


The following variables are unknown:

$$
T_{t}, R_{1}, R_{2}, \ell_{1} x_{1}, x_{2}, y_{0}, y_{1}, y_{2}, \phi_{1}, \phi_{2}, \psi_{1}, \ell_{1}, \ell_{2}, \ell_{3}
$$

In principle, the fifteen equations can be solved simultaneously to predict the unique trunk shape for the given known quantities.

Equations (4-23) and (4-25) may be solved simultarieously for $x_{2}$. The result is:

$$
x_{2}=\sqrt{-v_{0}^{2}-2 R_{2} y_{0}}
$$

It may seem from geometry that $\mathrm{x}_{2}$ should always be positive; consequently, only the positive sign of the squre root in Equation (4-35) was chosen.

Similarly, Equations (4-24) and (4-26) may be solved simultaneously for $x_{1}$. The result is:

$$
x_{1}=a+(\text { sign }) \sqrt{R_{1}^{2}-\left(y_{0}+R_{1}-b\right)^{2}}
$$

The choice of sign on the square root in Equation (4-36) will depend upon whether $x_{1}$ falls to the right or to the left of $a$. The criteria for this sign will be treated later.

The process for solving the equation will now be outlined. The known variables are:

$$
a, b, l_{0}, E_{t}, p_{c} / p_{j}, Y_{o}
$$

The iterative process requires the assumption of $R_{1}$ and a determination of the sign in Equation (4-36) to provide a trial solution. Criteria for $R_{1}$ selection and sign will be given later.

(1) Assume $R_{1}$ value and determine sign.

(2) From Equation (4-1) compute $T_{\mathrm{t}}$. 


$$
T_{t}=p_{j} R_{1}
$$

(3) From Equation (4-3) compute $l$.

$\ell=\ell_{0}+\frac{T_{t}}{E_{t}} \ell_{0}$

14) Calculate the length of $\ell_{2}$ as follows:

$$
\begin{aligned}
& \mathrm{y}_{\mathrm{o}}=-\mathrm{Y}_{\mathrm{o}} \\
& \mathrm{R}_{2}=\frac{\mathrm{R}_{1}}{1-\mathrm{p}_{\mathrm{c}} / \mathrm{p}_{\mathrm{j}}} \\
& \mathrm{x}_{2}=\sqrt{-\mathrm{y}_{0}^{2}-2 \mathrm{R}_{2} \mathrm{y}_{0}} \\
& \mathrm{y}_{2}=\mathrm{R}_{2}+\mathrm{y}_{\mathrm{o}} \\
& \phi_{2}=\arctan \mathrm{x}_{2} \\
& \ell_{2}=\mathrm{R}_{2} \phi_{2}
\end{aligned}
$$

(5) Calculate the length of $\ell_{1}$ as follows:

$$
\begin{aligned}
& x_{1}=a+(\operatorname{sign}) \sqrt{-\left(y_{0}+R_{1}-b\right)^{2}+R_{1}^{2}} \\
& y_{1}=R_{1}+y_{0} \\
& \psi_{1}=\arctan \frac{b-v_{1}}{a-x_{1}} \quad-\frac{\pi}{2} \leqslant \psi_{1} \leqslant 3 \frac{\pi}{2}
\end{aligned}
$$




$$
\begin{aligned}
& \phi_{1}=\frac{\pi}{2}+\psi_{1} \\
& \ell_{1}=\mathrm{R} \phi_{1}
\end{aligned}
$$

(6) Calculate the length of $l_{3}$ as follows:

$$
\ell_{3}=\left|x_{1}-x_{2}\right|
$$

(7) Calculate the difference between the trial solution for $\bar{\ell}$ in Equation (4-31) and the value of $\ell$ from Equation (4-3). The result is:

$$
\bar{l}-\ell=\epsilon
$$

If $\varepsilon$ approaches zero in Equation (4-37), the correct values of all the variables can be obtained. It should be noted that both $\bar{\ell}$ and $\ell$ are complicated functions of $R_{1}$.

(8) Iterate the procedure until $E$ in Equation (4-37) approaches zero to the accuracy desired.

In order to develop the desired solution to the system of equations, numerical methods using Mueller's algorithm (Appendix I) may be used. Mueller's algorithm converges on the root of a complicated function, such as those specified in Equation (4-37), by approximating the function with a second degree polynomial. In order to apply Mueller's algorithm, it is necessary to bracket the desired root of Equation (4-37).

Therefore, it is desired to determine two values of $R_{1}$ which will bracket the desired root in Equation (4-37). The value of $R_{1}$ which provides the upper bracket (makes 
$\epsilon$ positive in Equation (4-37)) will be designated $\left(R_{1}\right)_{U}$. The value of $R_{1}$ which provides the lower bracket (makes a negative) will be designated as $\left(R_{1}\right)_{L}$.

The technique for determining the lawer bracket $\left(R_{1} l_{L}\right.$ will now be considered.

For a given $Y_{0}$ and $b$, the minimum value which $R_{1}$ can assume land yet be tangent to the ground line) is illustrated in Figure 4-5.

From Figure 4-5, it is evident that the minimum $R_{1}$ is:

$$
\left(R_{1}\right)_{\text {MIN }}=\frac{b-y_{0}}{2}
$$

As a first trial, let $R_{1 L}=\left(R_{1}\right)_{M 1 N}$.

A check to determine if $\left(R_{1}\right\rangle_{\text {MIN }}$ provides a suitable lower bound can then be made. Steps 1 through 4 of the iteration process can be performed to calculate the value of $\ell_{2}$. However, in order to calculate $\ell_{1}$ the sign must be determined. The sign value is determined by comparing the actual trunk length with the trunk length associated with $\left\langle R_{1}\right\rangle_{\text {MIN. }}$

The value of $\ell$ associated with $\left(R_{1}\right)_{\text {MIN }}$ is designated $\ell_{4}$ and is calculated from geometry.

$$
\ell_{4}=\ell_{1}+\ell_{2}+\ell_{3}=\frac{\pi\left(b-\gamma_{0}\right)}{2}+\phi_{2} R_{2}+\left|a-x_{2}\right|
$$

In Equation (4-39), $\ell_{4}$ is the minimum trunk length associated with the condition $R_{1}=\left(R_{1}\right)_{\text {MIN }}$ under the restrictions that $x_{1}>x_{2}$ and $x_{2}>0$. It should be noted that $\ell_{4}$ is not necessarily the minimum trunk length for all values of $R_{1}$.

The value of $\ell_{4}$ is represented in Figure 4-5(a) (for $\mathrm{p}_{c} / \mathrm{P}_{\mathrm{j}}=0$ ). The fact that $\ell_{4}$ is not the minimum trunk length for all values of $R_{1}$ is illustrated in Figure $4-5(b)$. It is evident from the figure that the trunk length $\left(\ell_{4}\right)$ associated with $\left(R_{1}\right)_{M 1 N}$ is greater than 


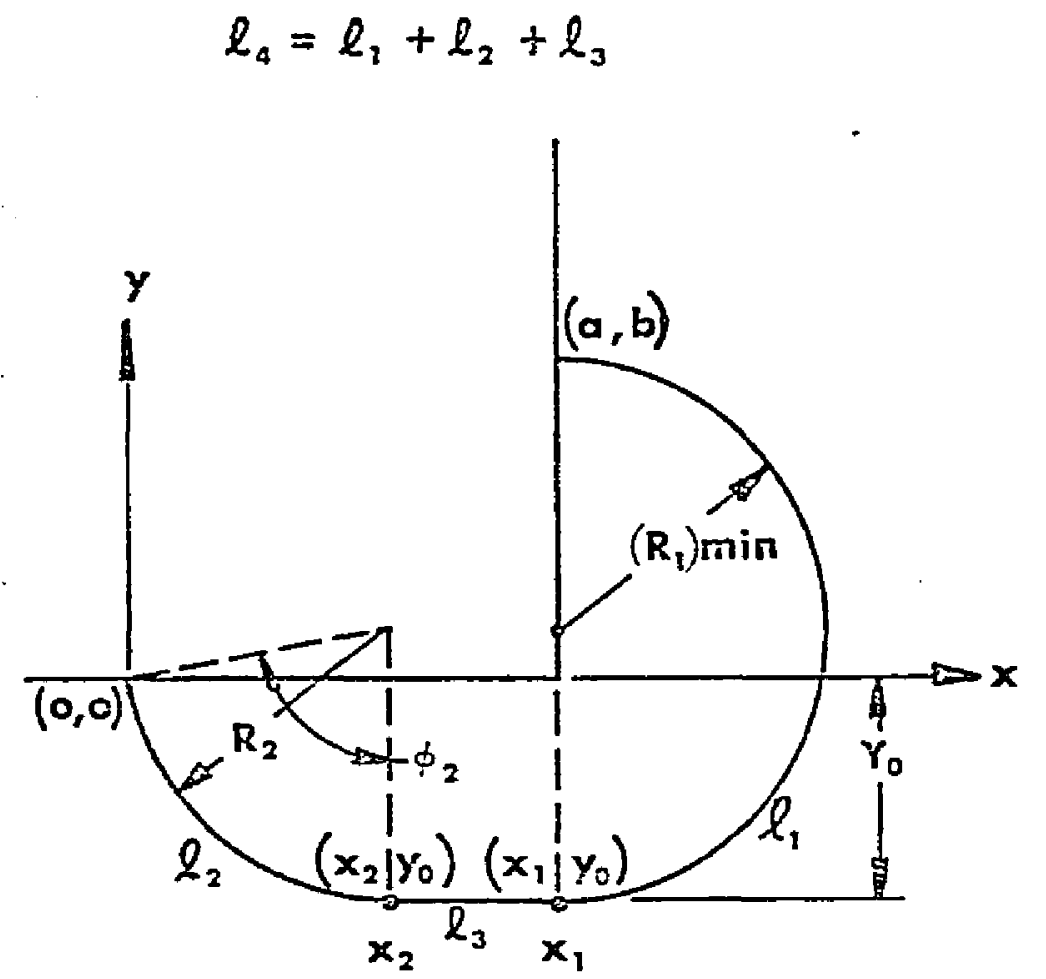

NOTE: $\left(R_{1}\right) \min$ DOES NOT ALWAYS CORRESPOND TO $(\ell)_{\min }$

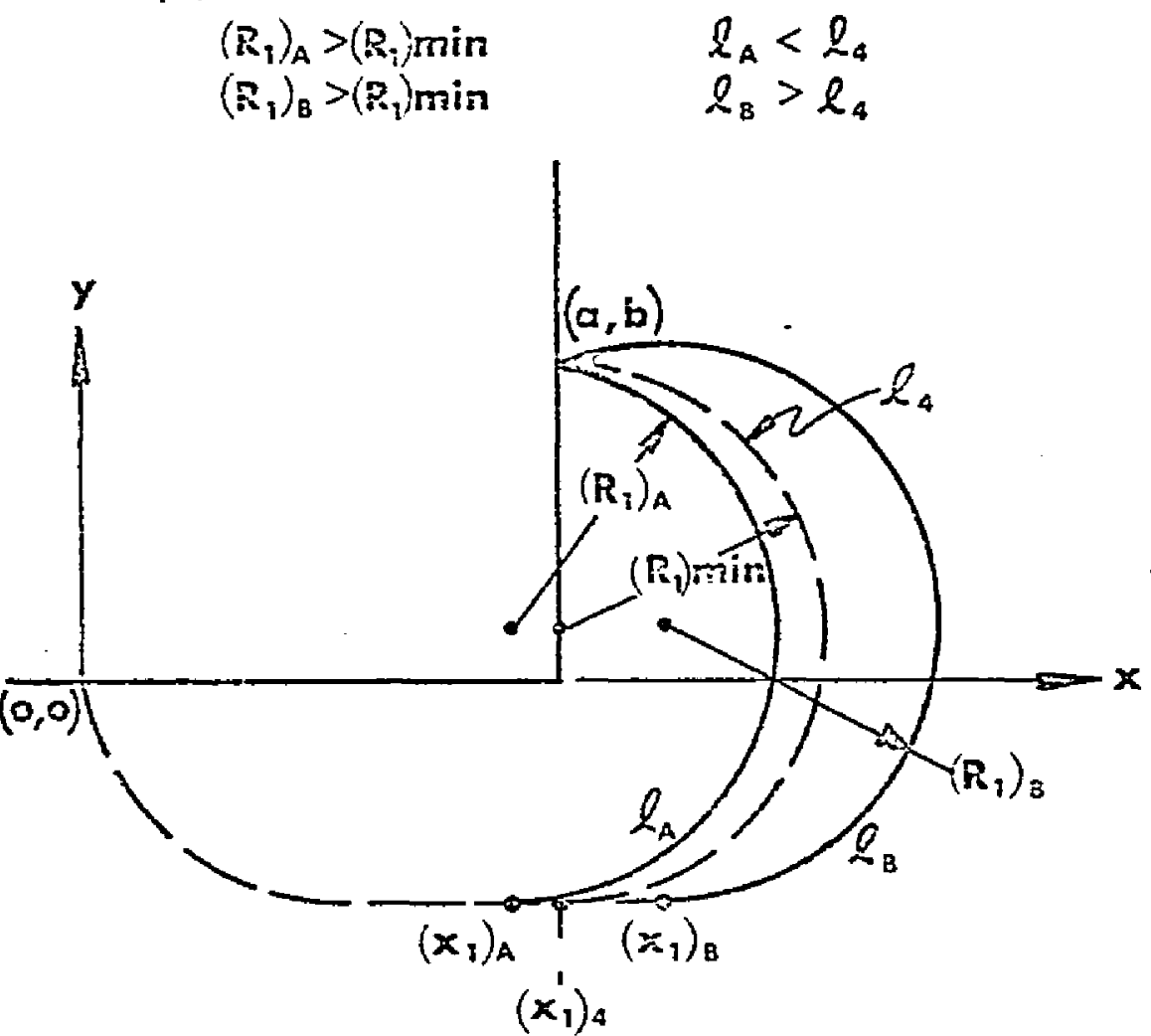

(a)PHYSICAL REPRESENTATION OF $l_{4}$

(b) PHYSICAL REPRESENTATION OF VARIOUS VALUEs of $R_{1}$

ILLUSTRATION OF MINIALZM TRUNK LENGTH FIGURE 4-5 
the trunk length $\left(\ell_{A}\right)$ associated with $\left(R_{1}\right)_{A}$. Further, whenever $x_{1} \geqslant a$, then $\ell>\ell_{4}$ for all

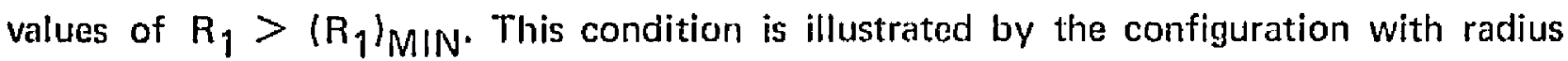
$\left\langle R_{\boldsymbol{\gamma}}\right\rangle_{\mathrm{B}}$ in Figure 4-5(b).

As illustrated above, the value of $\left(R_{1}\right)_{\text {MIN }}$ is a satisfactory lower bracket for the solution if $\ell>\ell_{4}$ and $x_{1}>x_{2}$. In this case $x_{1} \geqslant a$, and the sign in Equation (4-36) is plus.

The upper bracket for the condition $\ell>\ell_{4}$ may be found from the geometry of Figure 4-6. This figure shows the maximum value of $R_{1}$ possible for given values of $a, b$, and l.

The length of the chord between coordinates $(0,0)$ and $(a, b)$ in Figure 4.6 may be written in terms of the radius and central angle or in terms of the rectangular coor dinates. If the two expressions are equated, the result is:

$$
\sqrt{a^{2}+b^{2}}=2 R_{1} \sin \frac{\phi_{1}}{2}
$$

Further, the radius, arc length and central angle are related as follows:

$$
\phi_{1}=\frac{\ell}{R_{1}}
$$

These two relationships may be combined to give a relationship for $R_{1}$.

$R_{1} \sin \frac{\ell}{2 R_{1}}=\sqrt{\frac{a^{2}+b^{2}}{4}}$

Equation (4.40) may be solved numerically to give the upper bracket $\left(R_{1}\right)_{U}$ for the condition $\ell>\ell_{4}$.

It is now necessary to consider the upper and lower brackets for the condition $\ell$ $<\ell_{4}$. Two cases are possible. The first is the condition $x_{1}<x_{2}$. The second is the 


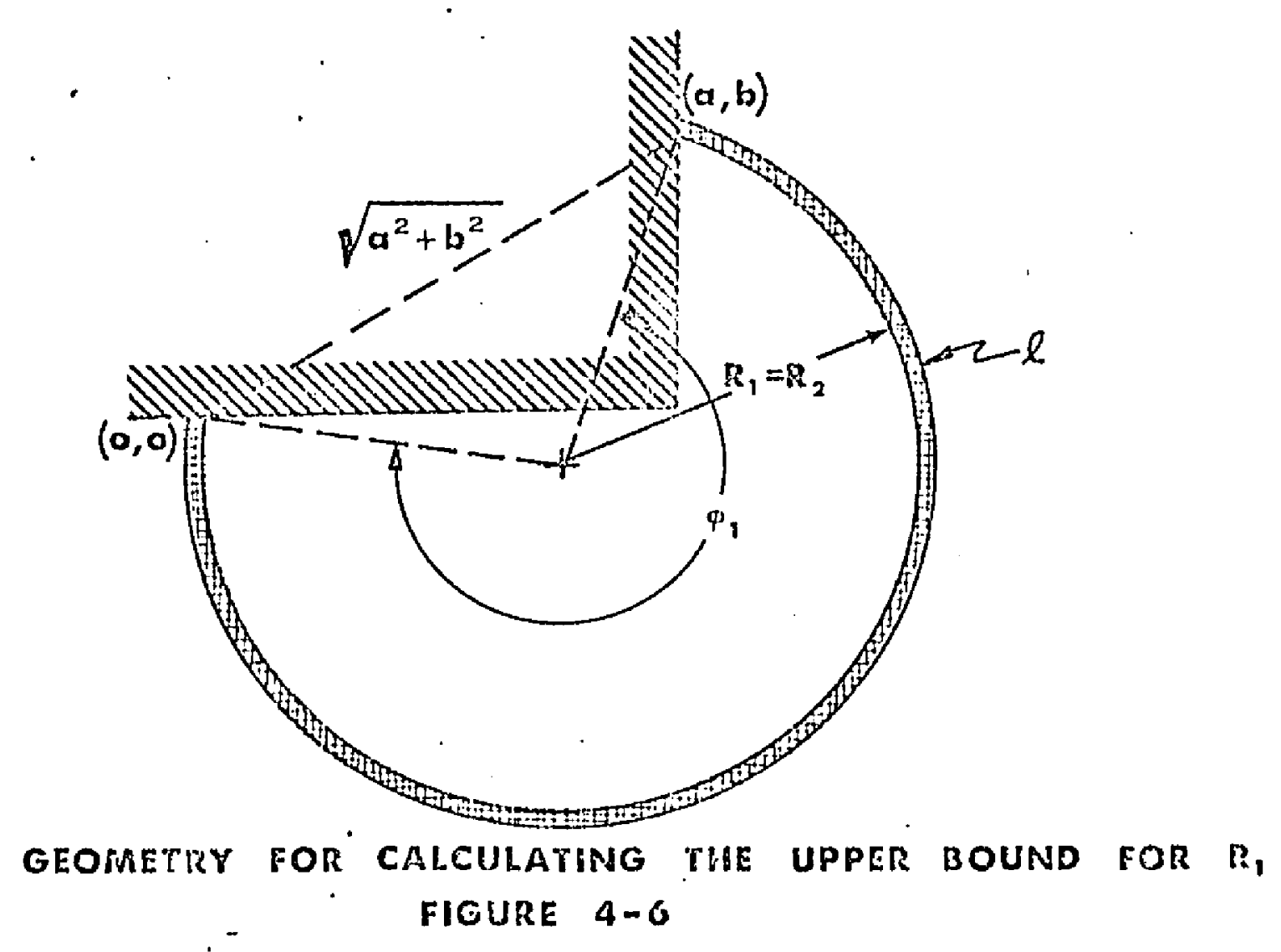




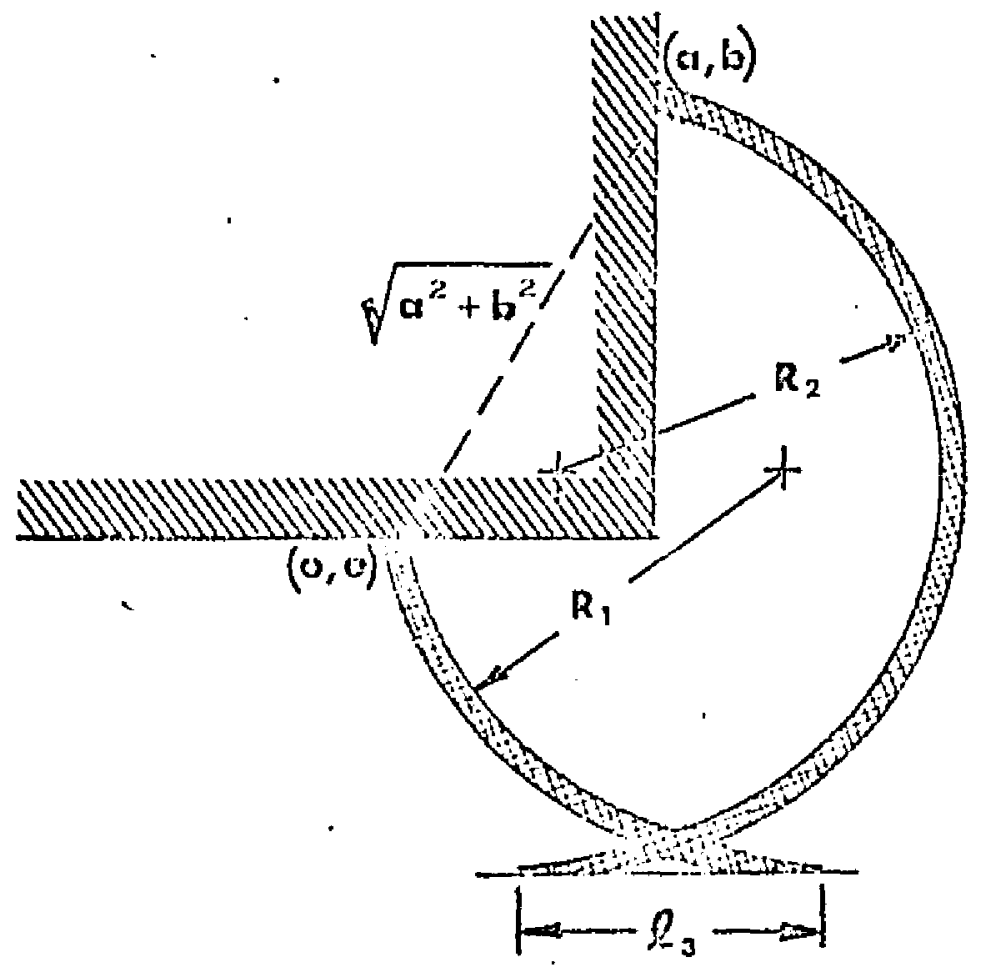

\section{PHYSICALLY IMPOSSIBLE SOLUTION FIGURE $4-7$}


condition $x_{1}>x_{2}$. In the first case; the condition shown in Figure 4.7 exists. This case is of no practical interest and will not be considered.

If, on the other hand, $\ell<\ell_{4}$ and $x_{1}>x_{2}$, then from the geometry of Figure 4-5(b) it is evident that $x_{1}<a$. Therefore, in this case, the sign in Equation (4-36) is minus. Further, $\left(R_{1}\right)_{M I N}$ is not a satisfactory lower bracket for the solution of Equation (4-37). In this case, the correct value of $\ell$ lies between the configuration represented by $\left(R_{1}\right)_{A}$ and $\left(R_{1}\right)_{\text {MIN }}$ in Figure $4-5(b)$. Thereiore, under these conditions, $\left(R_{1}\right)_{M i N}=\left(R_{1}\right)_{U}$ forms a satisfactory upper bound.

It is necessary to establisly a difforent criteria for the lower bracket $\left(R_{1}\right)_{L}$ for the condition $\bar{l}<\ell_{4}$ and $x_{1}>x_{2}$. The minimum value possible for $\ell$ for given values of $a, b$, $p_{c}, p_{j}$, and $Y_{o}$ is reached when $\ell_{3}=0$ in Equation (4-31). This occurs when $x_{1}=x_{2}$. The value of $R_{1}$ for the condition $x_{1}=x_{2}$ establishes the lower bracket for the solution to Equation (4-36). This value occurs between the values of $x_{1}=0$ and $x_{1}=a$.

Numerically the upper bracket for $x_{1}=x_{2}$ is:

$$
\left(R_{1}\right)_{U}=\frac{b-v_{0}}{2}
$$

The lower bracket may be found by setting Equation (4-36) equal to zero and solving for $R_{1}$. The result is:

$$
\left(R_{1}\right)_{L}=\frac{a^{2}+b^{2}+y_{o}^{2}-2 y_{o} b}{2\left(b-y_{o}\right)}
$$

Using iterative numerical techniques (Mueller's method) it is now possible to solve for the $R_{1}$ associated with $x_{1}=x_{2}$. This $R_{1}$ is then taken as $\left(R_{1}\right)_{U}$ which is required to provide a solution to the system of equations which define the non-equilibrium trunk shape. 
4.6 Trunk Cross-Sectional Area

The cross-sectional area of the free and loaded trunk shapes are shown in Figures 4-1 and 4-2 respectively. The cross-sectional area of the loaded trunk shape (Figure 4-2) has been divided into five regions which are designated by Roman numerals. The areas of each of these regions may be calculated as follows:

(1) Region 1 is the area of the sector of the circle with radius $R_{2}$ and central angle $\phi_{2}$ less the area of the triangle with vertices at coorclinates $(0,0),\left(x_{2}, y_{2}\right)$ and $\left(x_{2}, 0\right)$.

$$
A_{1}=\frac{\phi_{2}}{2} \mathrm{R}_{2}^{2}-\frac{\mathrm{x}_{2} \mathrm{y}_{2}}{2}
$$

(2) Region II is the area of the rectangle with corners at coordinates $\left(x_{2}\right.$, $0),\left(x_{1}, 0\right),\left(x_{1}, y_{0}\right)$ and $\left(x_{2}, y_{0}\right)$.

$$
A_{11}=-l_{3} Y_{0}
$$

(3) Region III is the area of the sector of the circle with radius $R_{1}$ and central angle $\phi_{\eta}$.

$$
A_{I I I}=\frac{\phi_{1}}{2} R_{1}^{2}
$$

(4) Region IV is the area of the rectangle with corners at coordinates $(a, 0)$, $\left(x_{1}, 0\right),\left(x_{1}, y_{1}\right)$ and $\left(a, y_{1}\right)$.

$$
A_{I V}=\left(x_{1}-a\right) V_{1}
$$


(5) Region $V$ is the arca of the triangle with vertices at coordinates $(a, b)$, $\left(x_{1}, y_{1}\right)$ and $\left(a, y_{1}\right)$.

$$
A_{V}=\frac{1}{2}\left(x_{1}-a\right)\left(b-v_{1}\right)
$$

The total cross-sectional area may be determined by summing the five areas given by Equations (3.43) through (3.47). The result for the Loaded Trunk Shape is:

$$
\begin{aligned}
\left(A_{j}\right)_{\text {loaded }}= & \frac{\phi_{2}}{2} R_{2}{ }^{2}-\frac{x_{2} y_{2}}{2}-\ell_{3} y_{0}+\frac{\phi_{1}}{2} R_{1}^{2} \\
& +\left(x_{1}-a\right) y_{1}+\frac{1}{2}\left(x_{1}-a\right)\left(b-y_{1}\right)
\end{aligned}
$$

For the Free Trunk Shape, the cross-sectional area may be derived by simplifying Equation (4-48). A comparison of Figures $4-1$ and $4-2$ shows that for the Free Trunk Shape the following simplifications are possible:

$$
\begin{aligned}
& l_{3}=0 \\
& x_{1}=x_{2}=x_{0}
\end{aligned}
$$

The above simplifications when applied to Equation (4-48) give an expression for the cross-sectional area of the Free Trunk Shape. The result is:

$$
\begin{aligned}
\left(A_{j}\right)_{\text {free }}= & \frac{\phi_{2}}{2} R_{2}^{2}-\frac{x_{o} v_{2}}{2}+\frac{\phi_{1} R_{1}^{2}}{2}+\left(x_{o}-a\right) y_{1} \\
& +\frac{1}{2}\left(x_{o}-a\right)\left(b-y_{1}\right)
\end{aligned}
$$




\subsection{Analytical Results}

\subsubsection{Approach}

The trunk shape problems involve a large number of variables whose dimensions are length to the first power. A large number of nondimensional ratios result. Consequently, the use of nindimensional parameters is of little value in presenting the results of this problem. The approach will be to predict the shape for two trunk cross sections of a typical design and indicate how the general method could be applied to other designs.

The trunk dimensions may be scaled by holding two scale factors constant. These scale factors involve only the independent variables, and are defined as:

$$
\begin{aligned}
& \pi_{1}=\frac{a^{2}+b^{2}}{\ell} \quad \text { the trunk length parameter } \\
& \pi_{2}=\frac{b}{a^{2}+b^{2}} \quad \text { the trunk attachment parameter }
\end{aligned}
$$

Provided these factors remain constant, the other dimensions may be scaled linearly with $\ell$.

The design chosen for analysis is approximately $1 / 3$ scale relative to the size required for a 60,000 pound aircraft such as the $\mathrm{C}-119$. A drawing of the model is shown in Figure 4-8. This model is only 82 inches in fength whereas the true $1 / 3$ scale model should be around 150 inches in length. Except for the length dimension, all others are to the $1 / 3$ scale.

The side and end trunk cross sections of the model shown in Figure 4.8 were selected for detailed analysis. The dimensions of these two sections are summarized in Table 4-I. 
TABLE 4-1

Trurk Model Dimensions

\begin{tabular}{|c|c|c|c|c|}
\cline { 2 - 5 } \multicolumn{1}{c|}{} & \multicolumn{2}{c|}{ END TRUNK } & \multicolumn{2}{c|}{ SIDE TRUNK } \\
\hline QUANTITY & MODEL & FULI SCALE & MODEL & FULI SCALE \\
\hline $\mathrm{a}$ & 2.35 & 7.06 & 1.44 & 4.33 \\
\hline $\mathrm{b}$ & 0.00 & 0.00 & 1.00 & 3.00 \\
\hline 2 & 3.10 & 9.30 & 4.62 & 13.86 \\
\hline$\pi_{1}$ & 0.88 & 0.88 & 0.382 & 0.382 \\
\hline$\pi_{2}$ & 0.00 & 0.00 & 0.570 & 0.570 \\
\hline$\sqrt{\mathrm{a}^{2}+\mathrm{b}^{2}}$ & 2.35 & 7.06 & 1.755 & 5.28 \\
\hline
\end{tabular}




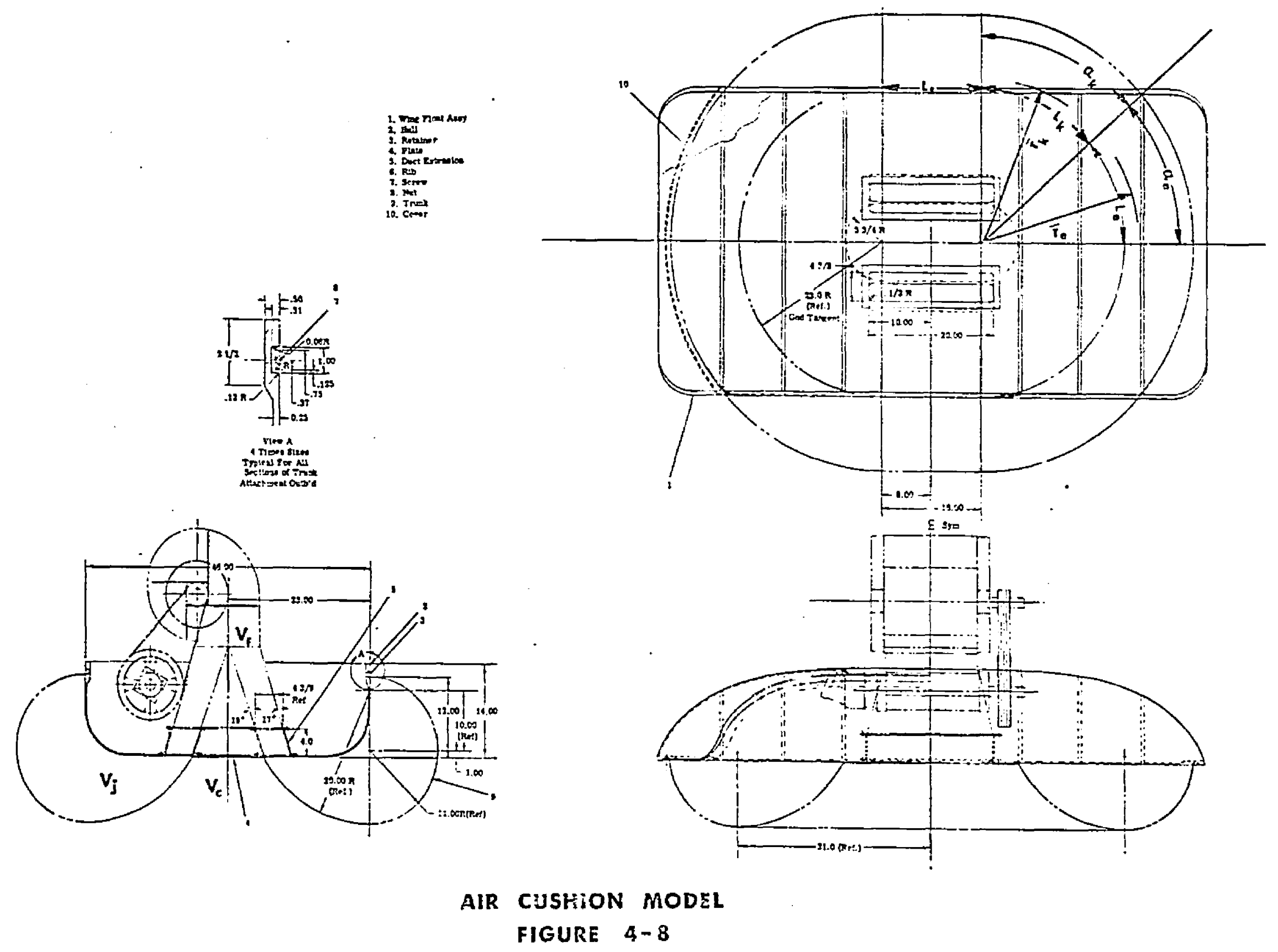




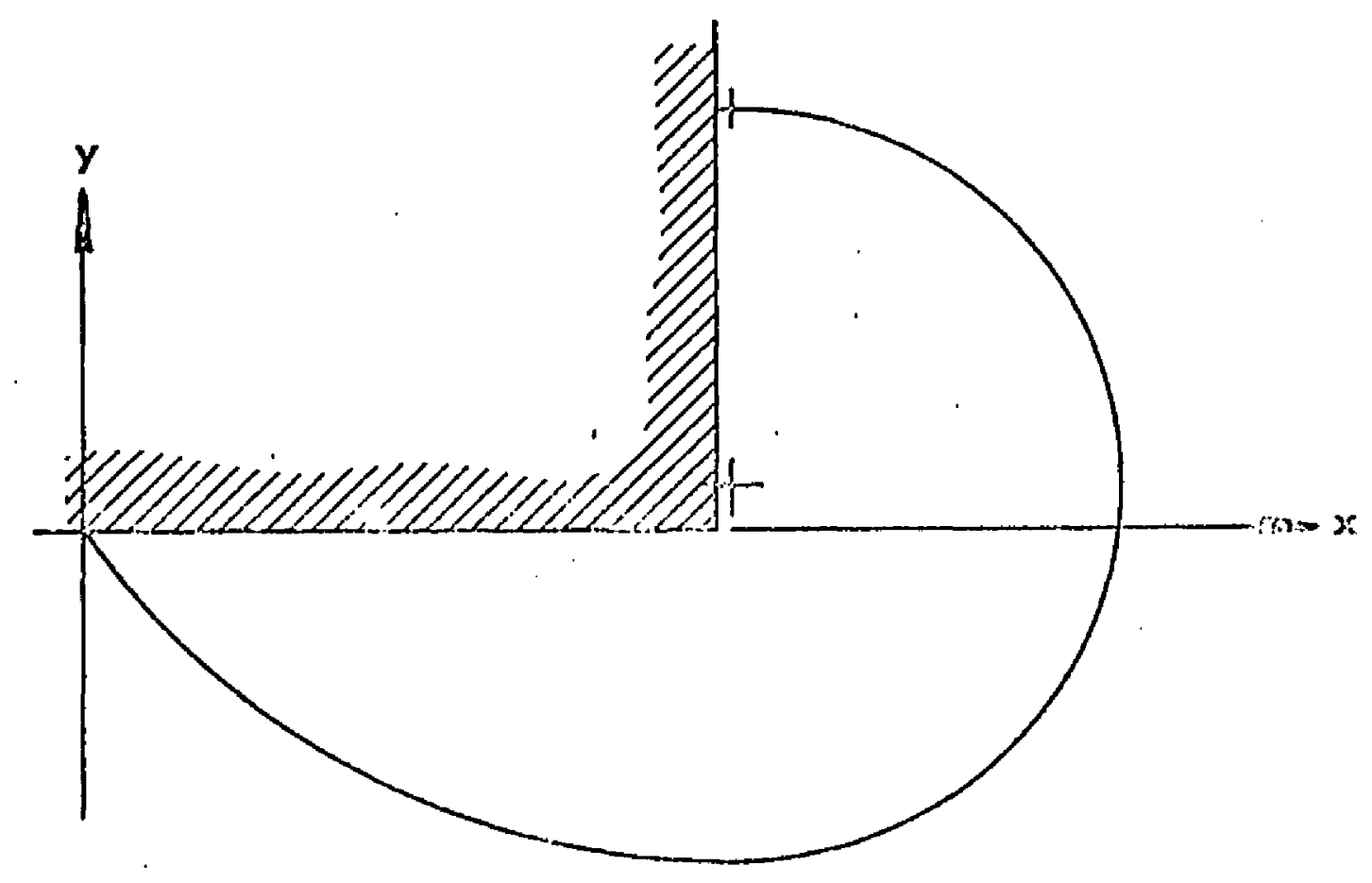

(a) SHAPE AT $P_{c} / P_{j}=0.5$

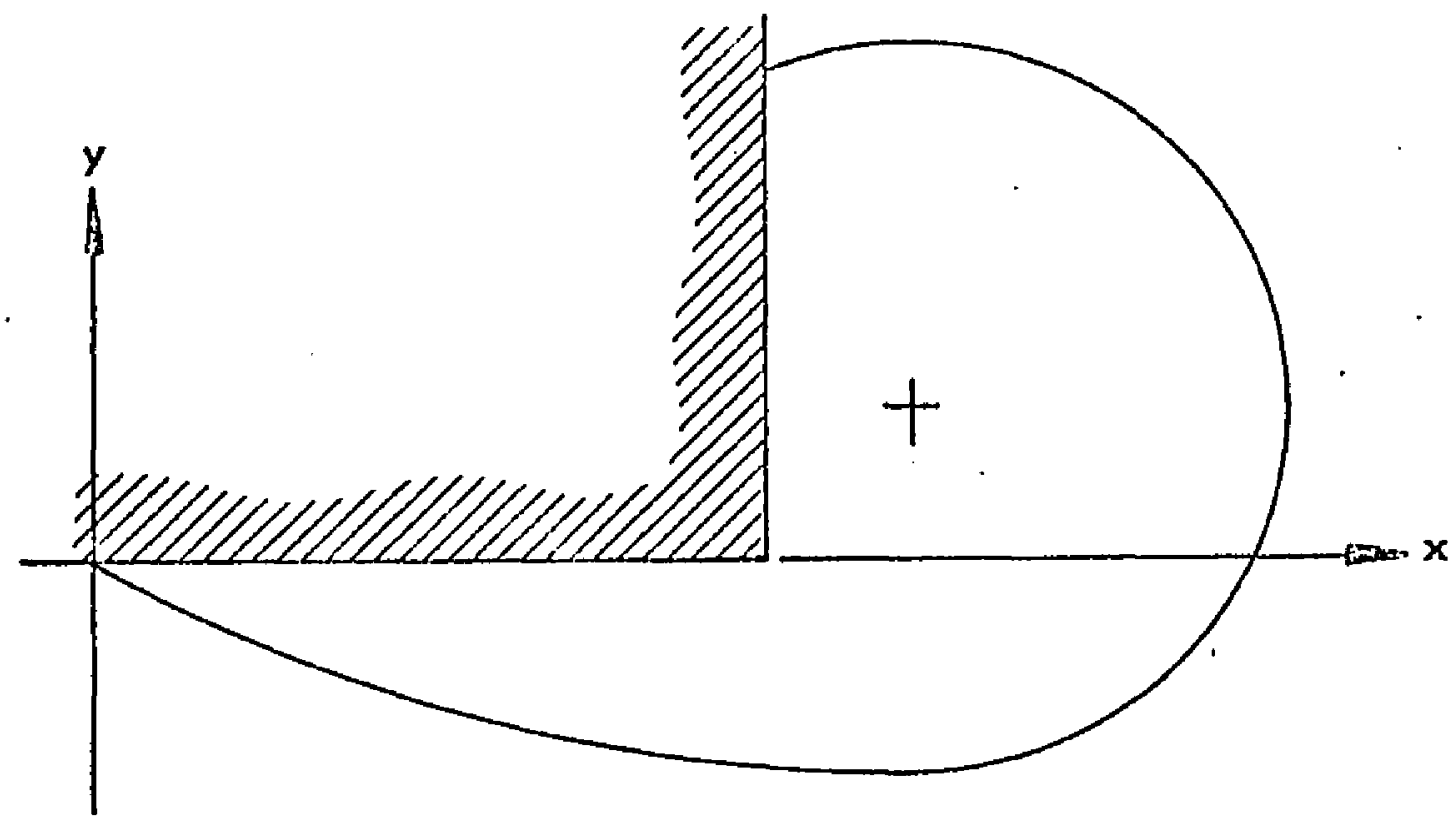

(b) SHAPE AT $P_{c} / P_{j}=0.8$ 


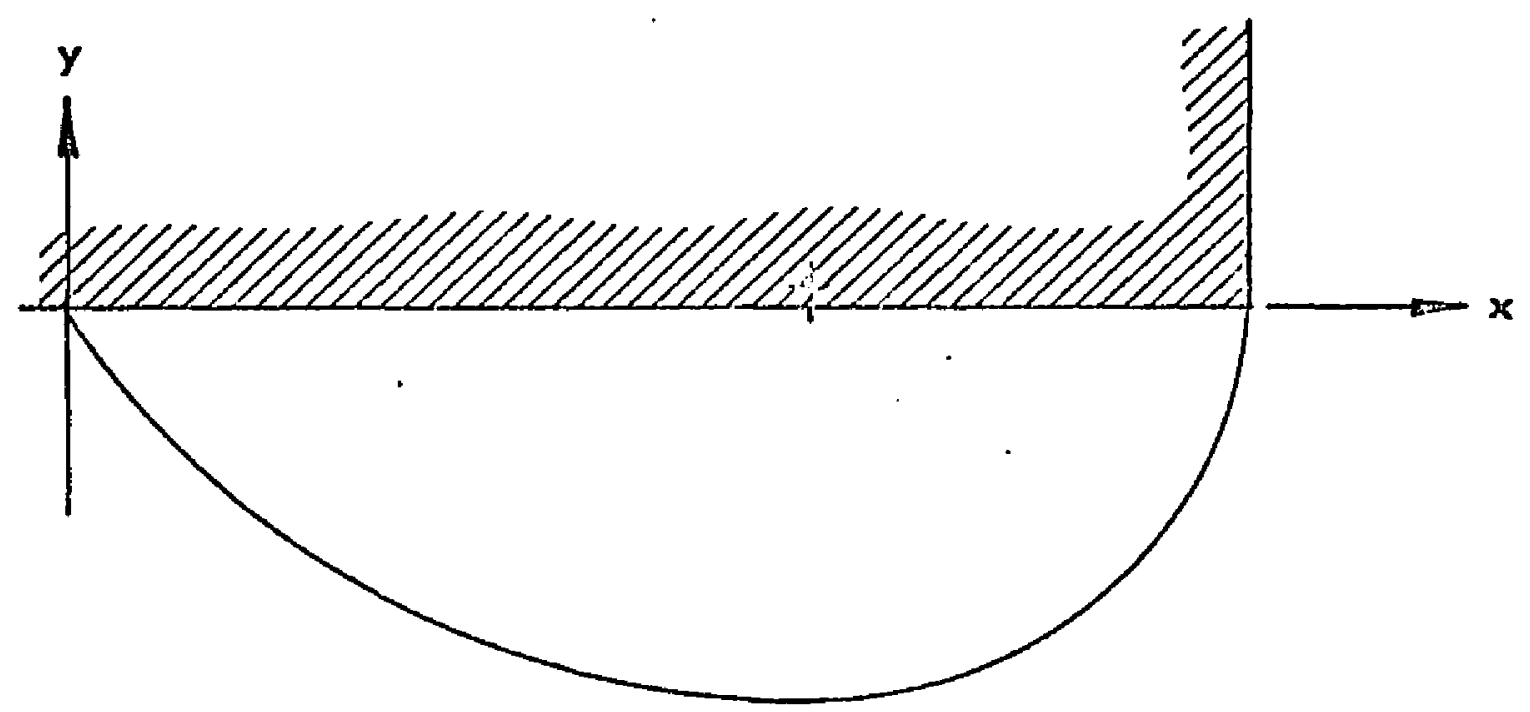

(a) SHAPE AT $P_{c} / P_{J}=0.5$

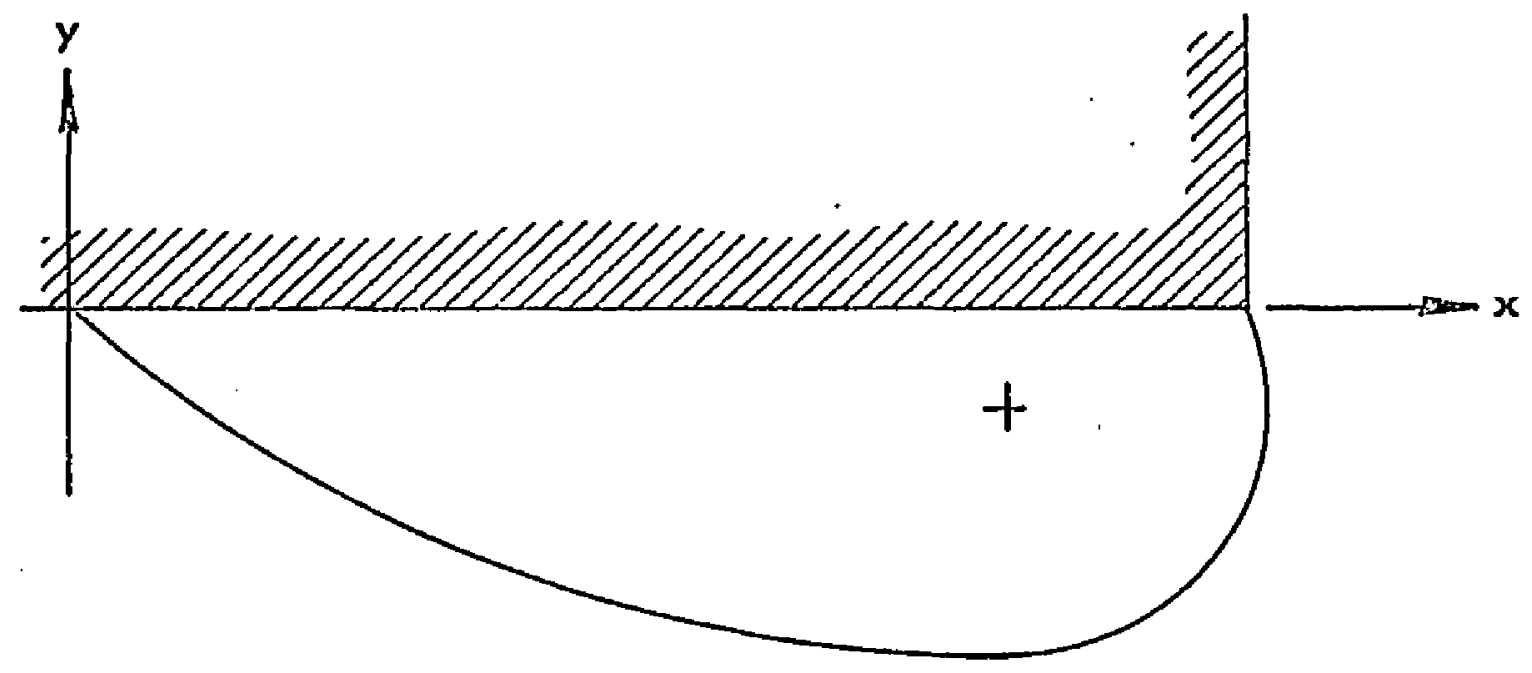

(b) SHAPE AT $P_{c} / P_{j}=0.8$

\author{
END TRUNK SHAPE \\ FIGURE 4-10
}


The analysis applied to the trunk shapes is the two-dimensional analysis developed in Sections 4.1 through 4.5 .

This analysis does not include the effect of loads and geometry changes perpendicular to the cross section shown in Figure 4-1.

It may be noted from the model drawing that the trunk cross-section at the sides is different from the cross section at the ends. This difference is caused by the necessity to pass the trunk under the fuselage to eliminate interference with the large cargo doors at the rear of the C-119. Most other military cargo aircraft also have a similar restriction.

\subsubsection{Free Trunk Shape Results (Inelastic Trunk)}

The cross-sectional shape of the trunk changes as $p_{c} / p_{j}$ varies. The effect of this change is illustrated pictoria!ly in Figures 4.9 and $4-10$ and graphically in Figures 4-11, 4-12, and 4-13.

The cross sections of the side trunk at $\mathrm{p}_{\mathrm{c}} / \mathrm{p}_{\mathrm{j}}=0.5$ and $\mathrm{p}_{\mathrm{c}} / \mathrm{p}_{\mathrm{j}}=0.8$ are shown in Figure 4-9. Figure 4.10 shows a similar relationship for the side trunk. It may be seen from these figures that an increase in $p_{c} / p_{j}$ iesults in a decrease in trunk height $\left(Y_{0}\right)$, a decrease in cross-sectional area $\left(A_{j}\right)$, and a shift to the outside for the ground tangent point $\left(x_{0}, y_{0}\right)$.

These qualitative effects are shown quantitatively in Figures 4-11, 4-12, and 4-13. These curves are developed from the computer program clescribed in Appendix I. .

Figure 4-11 shows the influence of $p_{c} / p_{j}$ on trunk height $\left(Y_{0}\right)$. The figure shows that there is a mismatch problem between the end trunk and the side trunk. The trunk was designed so that no mismatch would exist at $\mathrm{p}_{\mathrm{c}} / \mathrm{p}_{\mathrm{j}}=0.45$. At $\mathrm{p}_{\mathrm{c}} / \mathrm{p}_{\mathrm{j}}$ less than 0.45 the end trunk height is lower. At $\mathrm{p}_{\mathrm{c}} / \mathrm{P}_{\mathrm{j}}$ greater than 0.45 the side trunk height is lower. In practice, the mismatch shown is reduced by the clasticity of the trunk material.

Figure 4-12 shows the outward movement of the ground tangent point with increasing $\mathrm{p}_{\mathrm{c}} / \mathrm{p}_{\mathrm{j}}$. For a two dimensional model of the type shown in Figure $6-1$, the trunk 
103

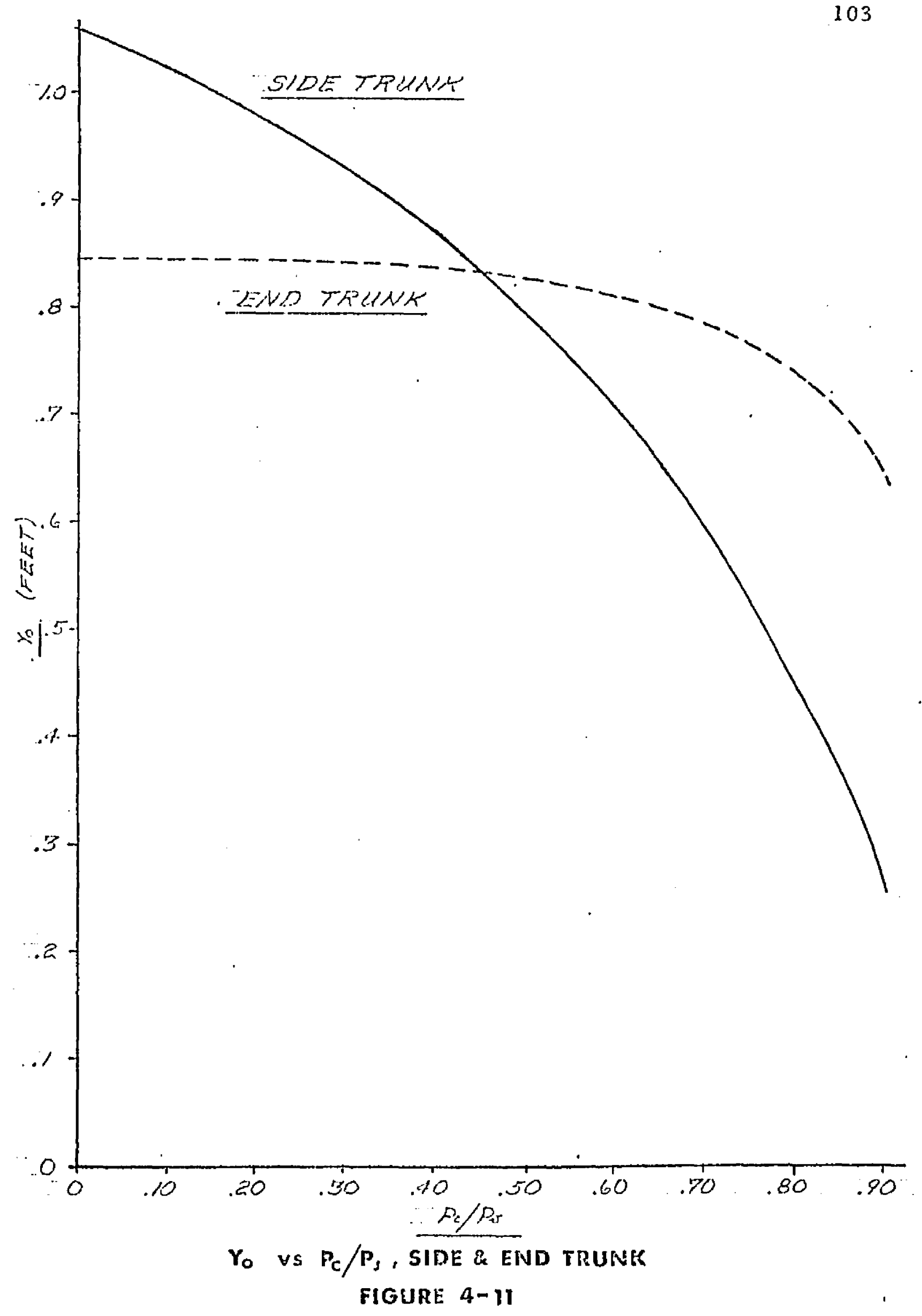




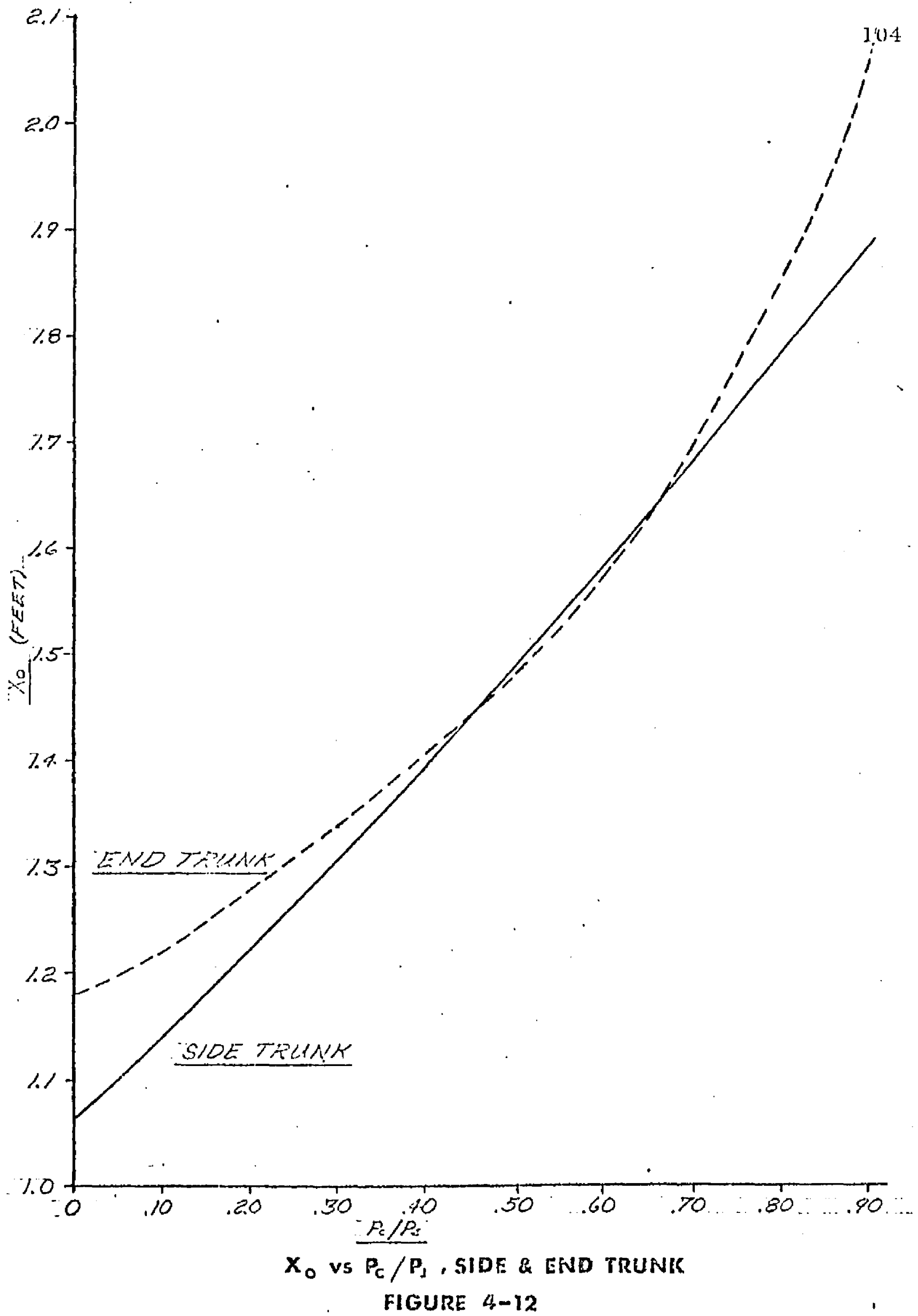




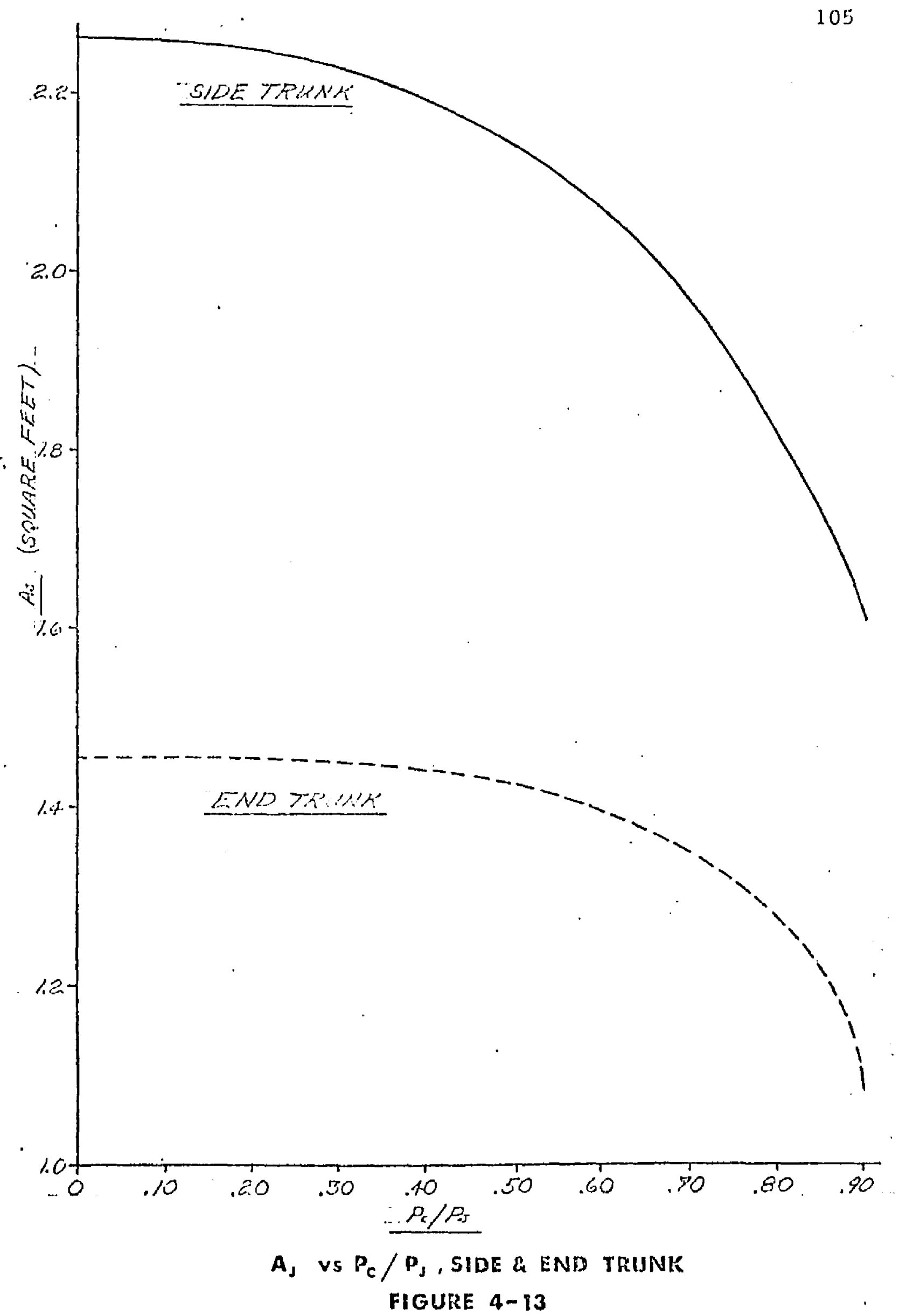


ends are unconstrained and the tangent point is free to move outward. However, for a three dimensional model of the type shown in Figure 4-8, no free edges exist and the trunk material must stretch to permit outward movement of the ground tangent. The actual trunk material envisioned for use on an air cushion landing system would be highly elastic (300\% stretch). Consequently, considerable movoment of $x_{0}$ should be permitted, and the two-dimensional predictions should be reasonable.

Figure 4-13 shows the variation in cross-sectional area with $\mathrm{p}_{\mathrm{c}} / \mathrm{p}_{\mathrm{j}}$ as predicted by Equation (3.48). The curve shows relative small area variation below $p_{c} / p_{j}=0.5$ and large variation above $\mathrm{p}_{\mathrm{c}} / \mathrm{p}_{\mathrm{j}}=0.5$.

\subsubsection{Free Trunk Shape Results (Elastic Trunk)}

The effect of using an elastic material for the trunk was investigated using the computer program described in Appendix III. The trunk material envisjoned is a rubber and nylon laminate. The nylon is laminated in a slack condition so that it does not carry load until the rubber has extended by at least $100 \%$. A typical elastic curve for such a material is shown in Figure 4-14. The material was selected so that at the design point $/ \epsilon=0, p_{c} / p_{j}=$ $0.5, p_{j}=80 \mathrm{psfg}$ ) the length of the elastic side trunk was equal to the length of the inelastic side trunk and the resulting shapes were identical. The effects of changing $\mathrm{p}_{\mathrm{c}} / \mathrm{p}_{\mathrm{j}}$ and $\mathrm{p}_{\mathrm{j}}$ on the shape of the side trunk and the end trunk constructed from the elastic material described by Figure 4-14 wore evaluated. The results are presented in Figures 4-15 through 4-20.

Figure 4-15 shiows the effect of $\mathrm{p}_{\mathrm{c}} / \mathrm{p}_{\mathrm{j}}$ on the trunk length. The effect of a $50 \%$ increase or decrease in the design pressure is also shown. The figure shows that the trunk length decreases with increasing $p_{c} / p_{j}$. The figure also shows that the trunk pressure has a large influence on the trunk length. The trunk material has a slack length of about 1.4 feet. At $p_{j}=80 \mathrm{psfg}$, the length has extended to around 4.9 feet. This large length change allows the trunk to elastically retract after take off to reduce aerodynamic drag. 


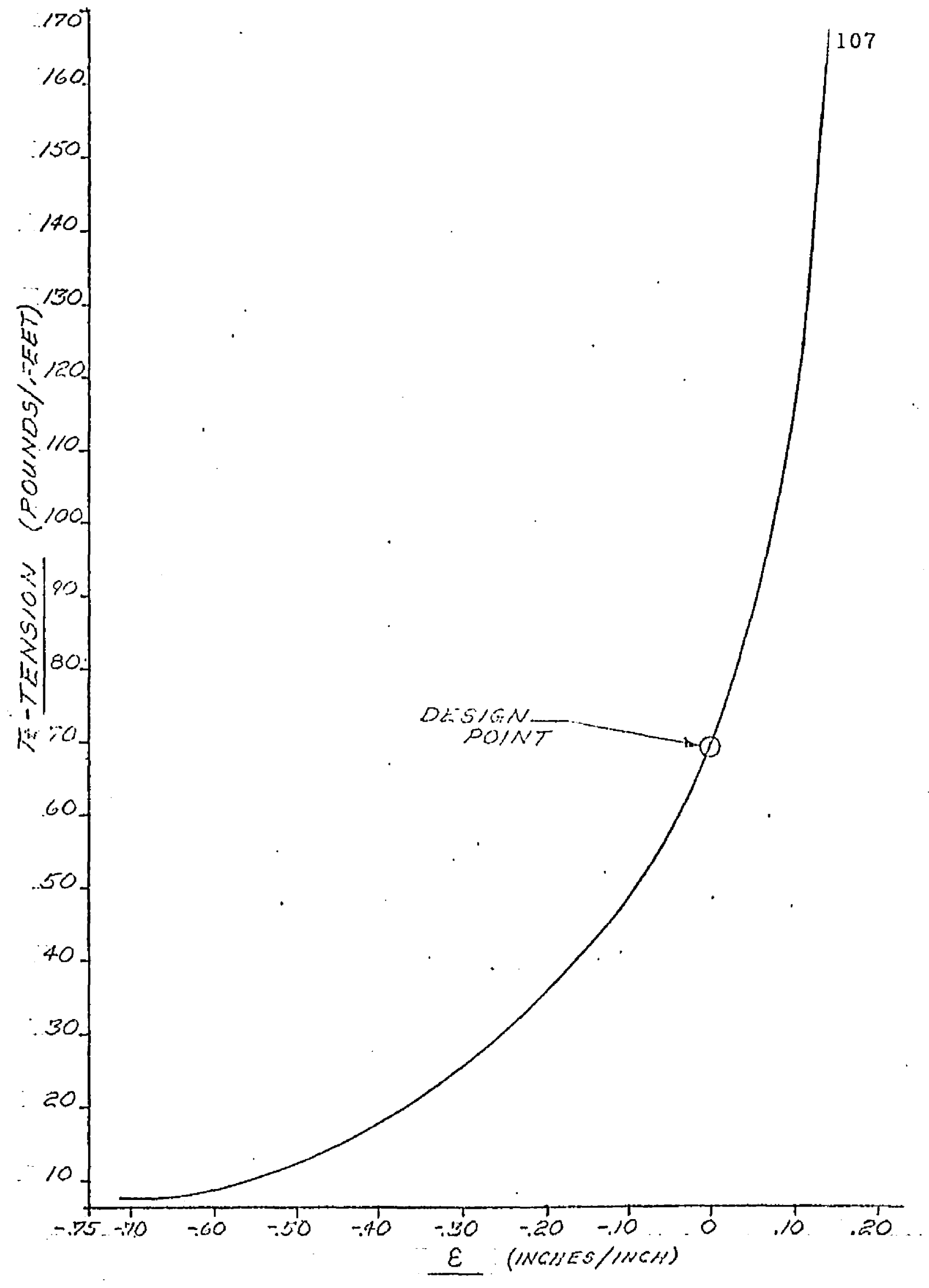

ELASTIC CURVE FOR YRUNK MATERIAL

FIGURE 4-14 


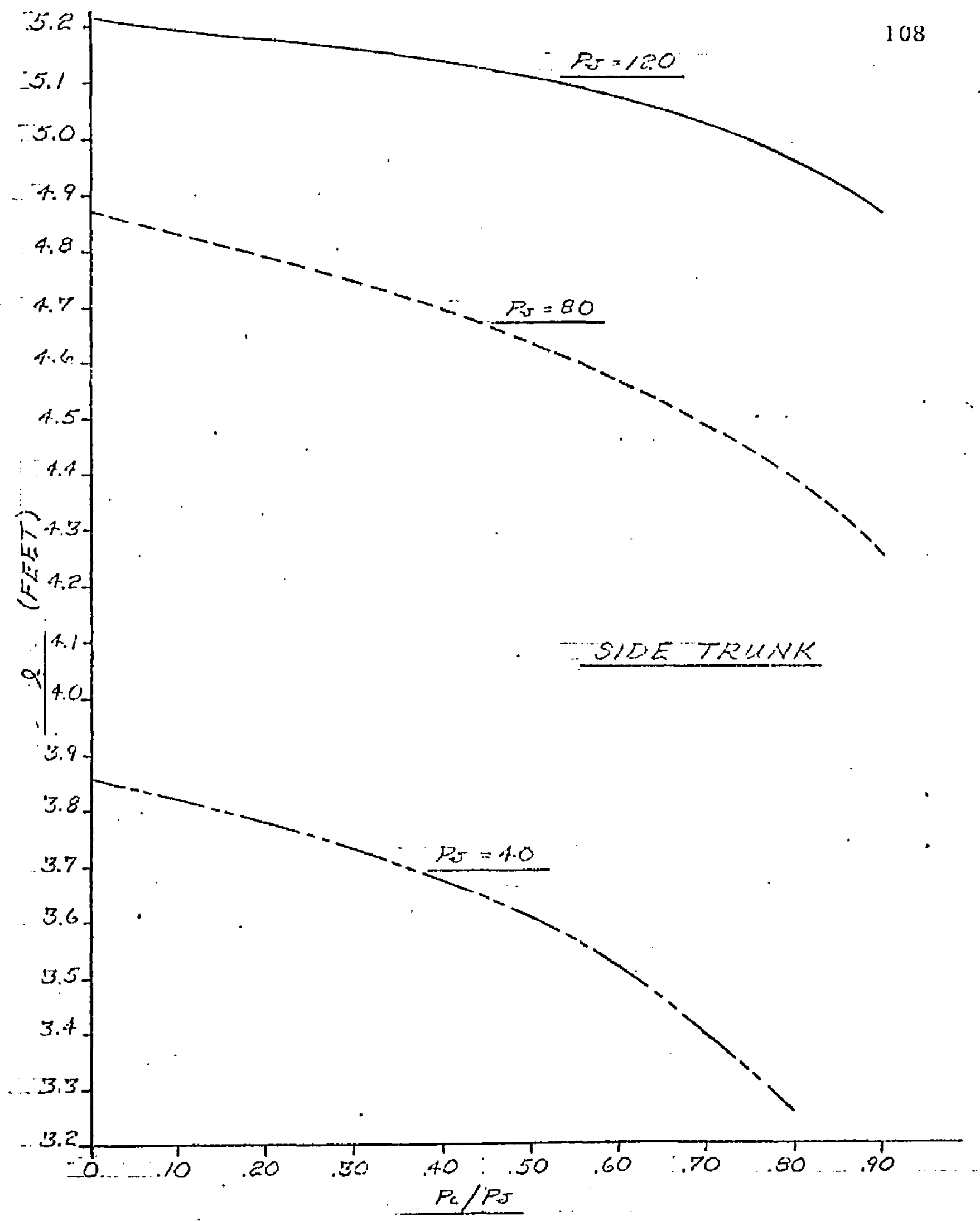

TRUNK LENGYH VS $P_{\mathrm{C}} / P_{\mathrm{J}}$, ELASTIC SIDE TRUNK

FIGURE $4-15$ 
Figures 4-16 and 4-17 show the trunk height for the side and end trunks, respectively. A comparison of the curves shows that the elastic trunk tends to reduce the mismatch problem. A comparison of $Y_{0}$ versus $\mathrm{P}_{\mathrm{c}} / \mathrm{p}_{\mathrm{j}}$ for the design trunk pressure $\left(\mathrm{P}_{\mathrm{j}}=80\right.$ psf(g) is shown in Figure 4-18. A comparison of Figure 4-16 and Figure 4-17 shows that the end and side trunk heights more nearly match for the elastic case than for the inelastic case.

The relationships between cross-sectional area $\left(A_{j}\right)$ and $p_{c} / p_{j}$ for the side and end elastic trunks are shown in Figures 4-18 and 4-19 respectively. The curves show that the cross-sectional area and consequently the trunk volume is very sensitive to changes in pressure below the design pressure $(80 \mathrm{psfg})$. The sensitivity to changes in pressure above the design pressure is not as great. The curve points out the necessity of carefully tailoring the material, design pressure combination to achieve the desired cross section. Errors in providing an excessively stiff material or insufficient $p_{j}$ could cause large degradation in the performance due to the large change in the trunk shape which would result.

A comparison of the trunk height for the elastic and inelastic side trunk is shown in Figure 4-20.

\subsubsection{Loaded Trunk Shape (Inelastic Trunk)}

The load support offered by the trunk is dependent upon the degree to which the trunk is flattened against the ground. This flattening is illustrated in Figure 4-2. The flattened length is characterized by $\ell_{3}$. Since this segment of the trunk membrane forms a straight line, the pressure on both sides of the membrane is assumed to be equal. The load support offered by the trunk is proportional to $\ell_{3}, p_{j}$ and the trunk depth(s).

The flattened length $\ell_{3}$ is dependent upon both $p_{c} / p_{j}$ and $Y_{0}$. For any value of $p_{c} / p_{j}$ there exists a value of $Y_{0}$ at which $\ell_{3}=0$. This value is the $Y_{\infty}$ for the equilibrium trunk shape case and is shown in Figure 4-11. When $Y_{0}$ is less than the $Y_{\infty}$ shown in Figure 4-11, trunk flattening occurs and $\ell_{3}$ has a positive value. The shape of the flattened trunk was evaluated using the computer program described in Appendix II. Some of the results are presented in Figures 4-21 through 4-24. 


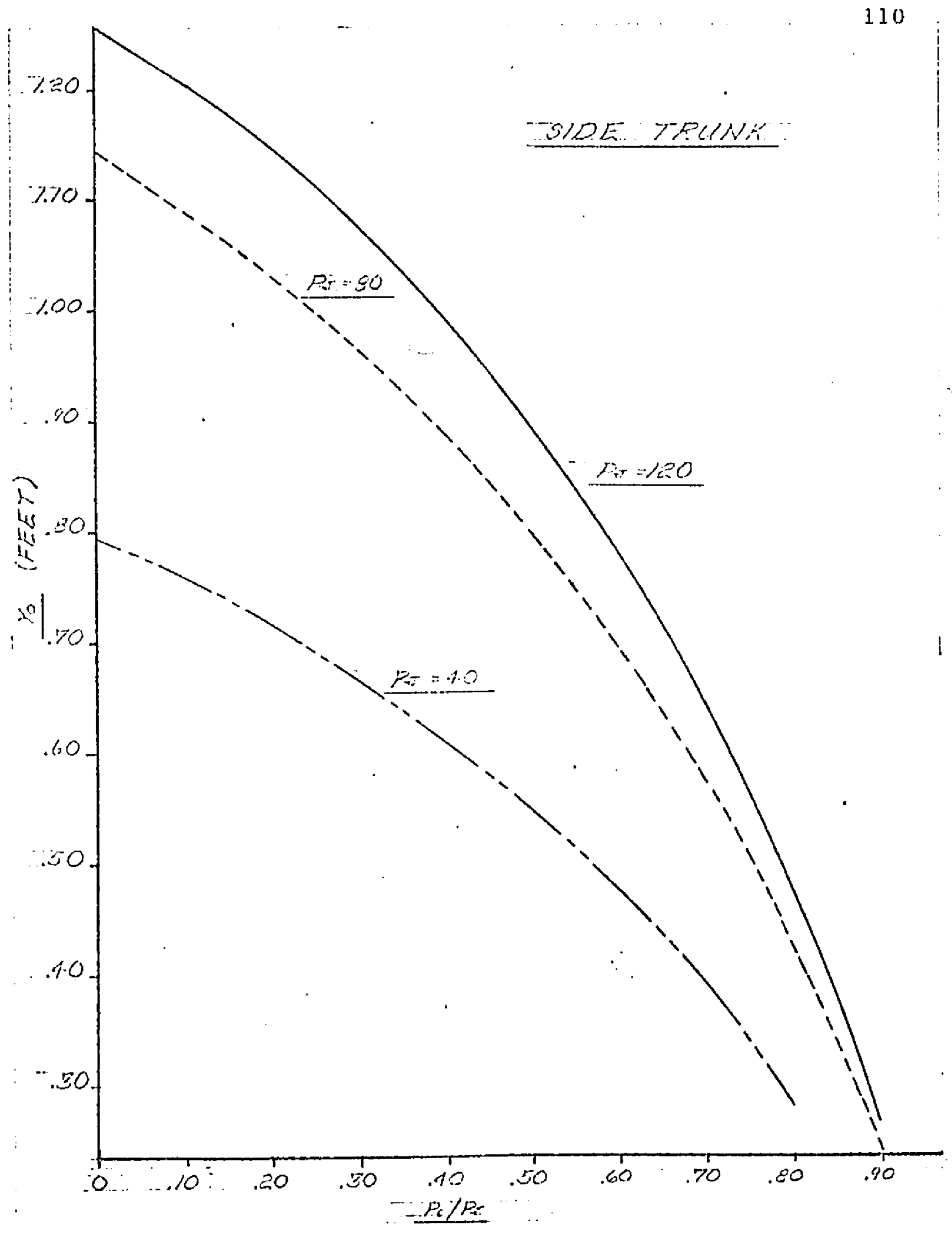

$Y_{0}$ vs $P_{c} / P_{J}$, ELASTIC SIDE TRUNK

FIGURE $4-16$ 
$\cdots P+/ 20$

.111

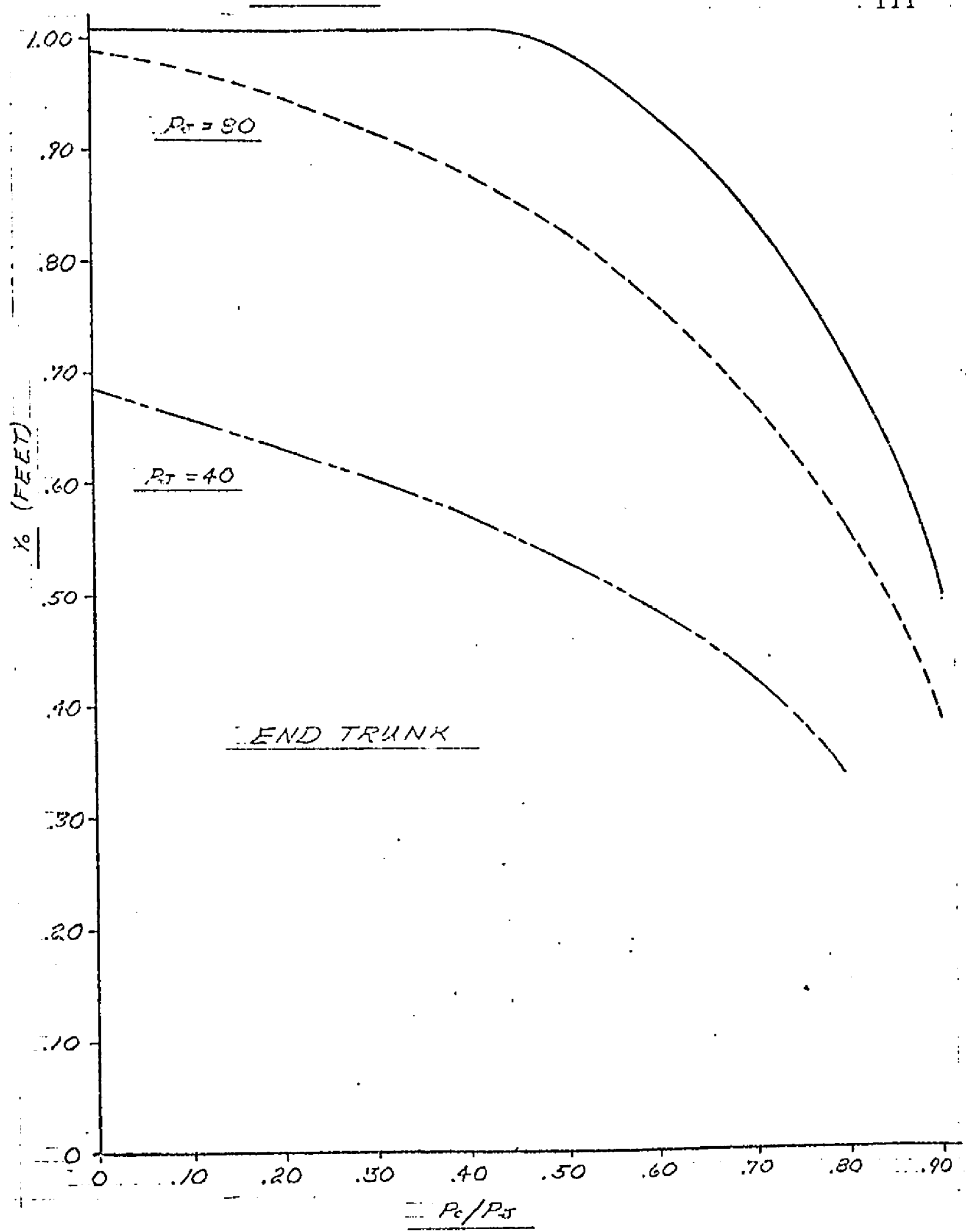

$$
Y_{0} \text { vs } P_{c} / P_{J} \text {, ELASTIC END TRUNK }
$$

FIGURE 4-17 


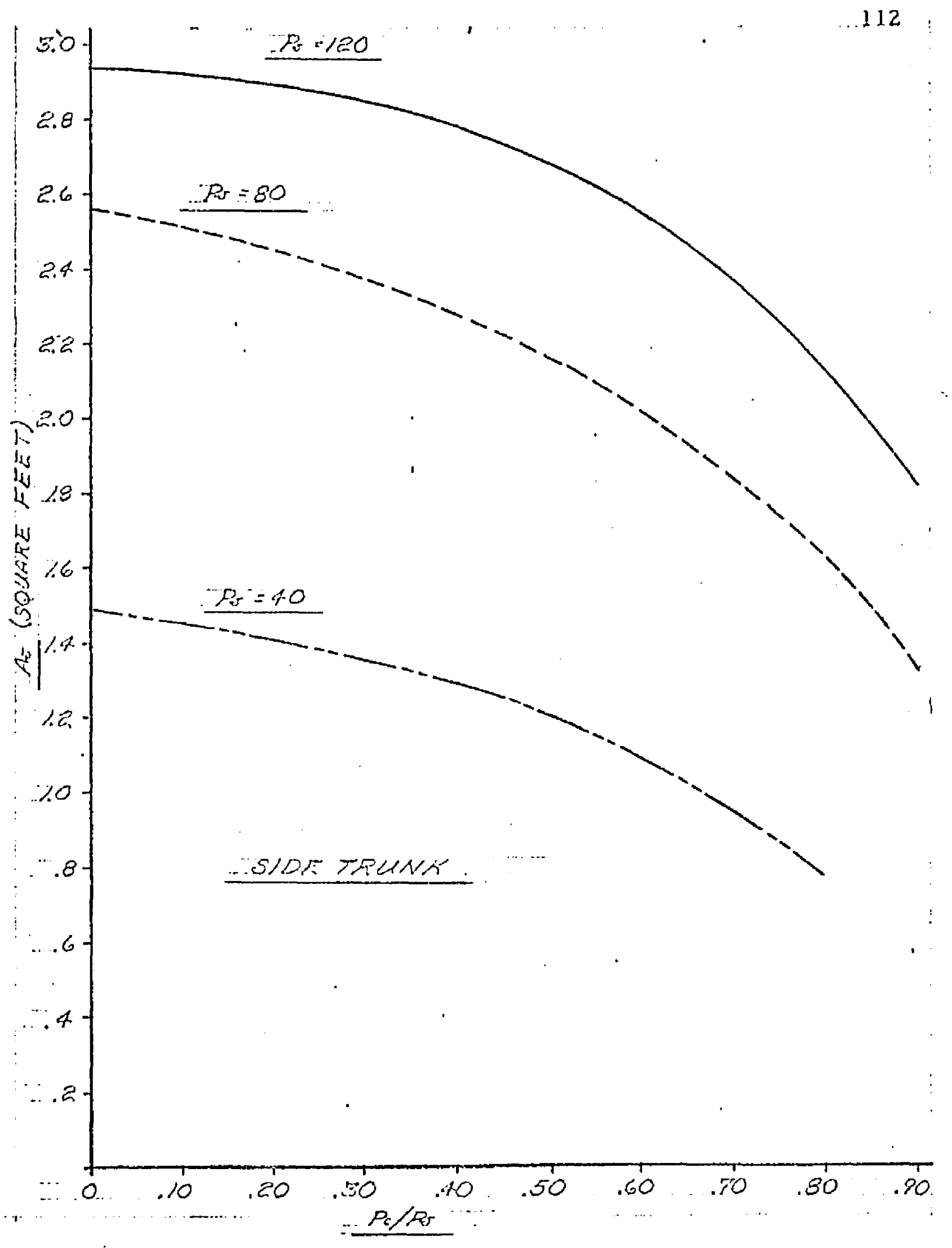




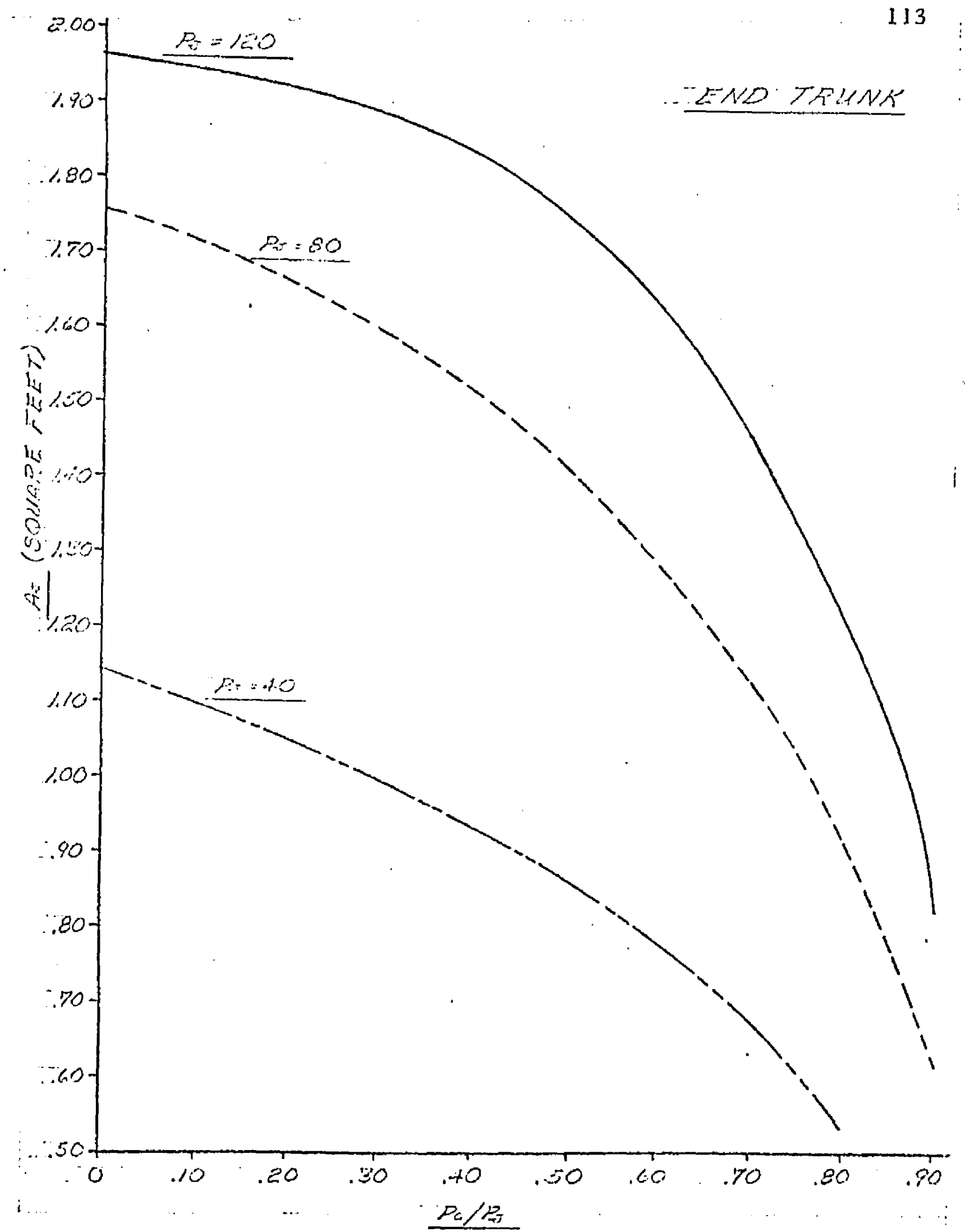

$$
\text { A, vs } P_{c} / P_{\jmath} \text {, ELASTIC END TRUNR }
$$

FIGUIRE 4-19 


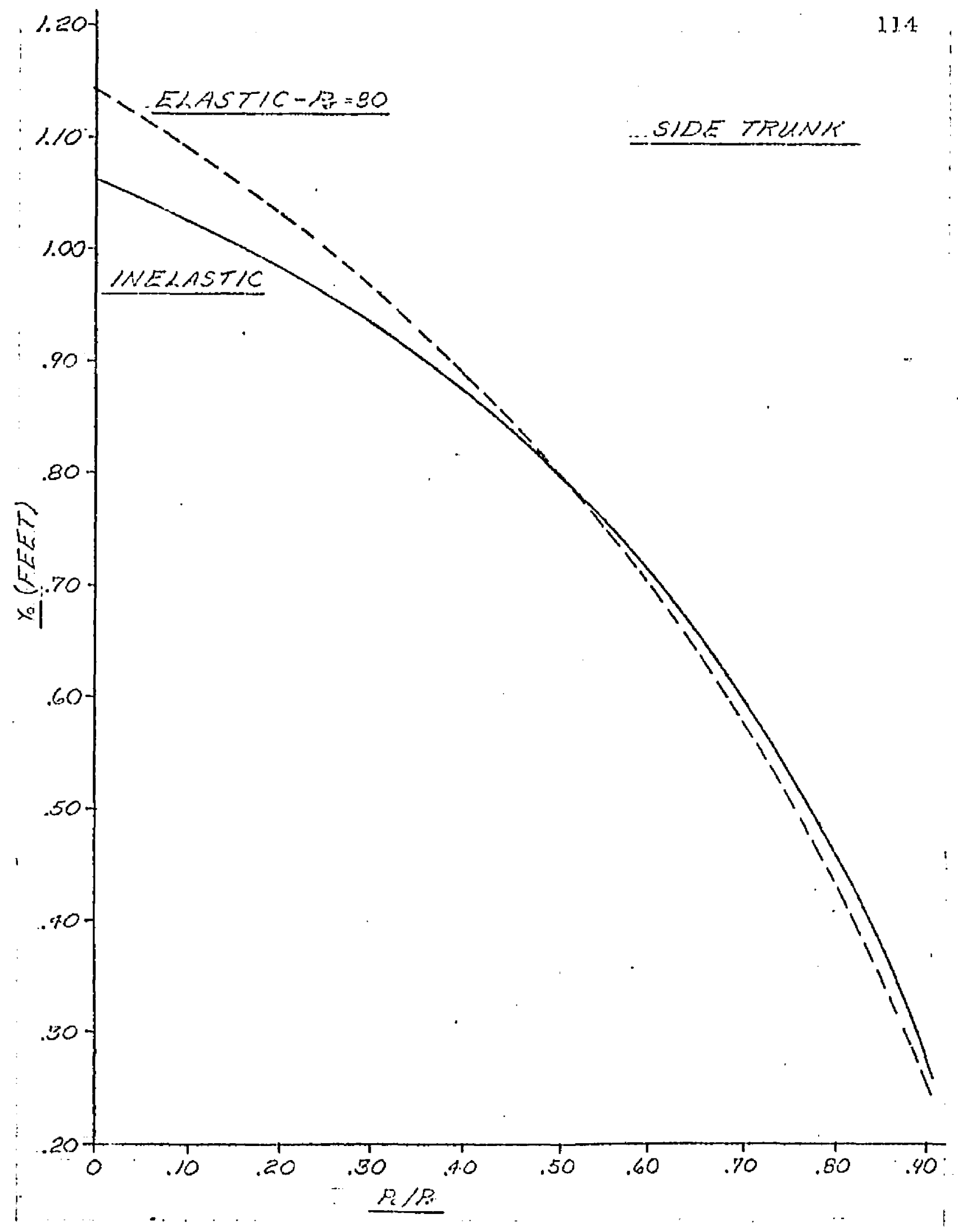

$\mathrm{Y}_{0}$ vs $\mathrm{P}_{\mathrm{c}} / P_{\mathrm{J}}$, COMPARISON OF RESULTS

FIGURE 4-20 
Figure 4-21 shows the relationship between $l_{3}$ and $Y_{0} / Y_{\infty}$ at various $p_{c} / P_{j}$ values for the side trunk. Figure 4.22 shows the same relationships for the end trunk. The slope of the $\ell_{3}$ versus $Y_{0} / Y_{\infty}$ curve is proportional to the stiffness. The curves show that the stifIness of both trunk shapes is nearly linear for deflections up to $50 \%$ of the free trunk height $\left(Y_{\infty}\right)$.

Figures 4-23 and 4-24 show the relationship between $A_{j}$ and $Y_{0} / Y_{\infty}$ for the side and end trunk respectively. The values of $A_{j}$ were predicted by Equation (3-48). 


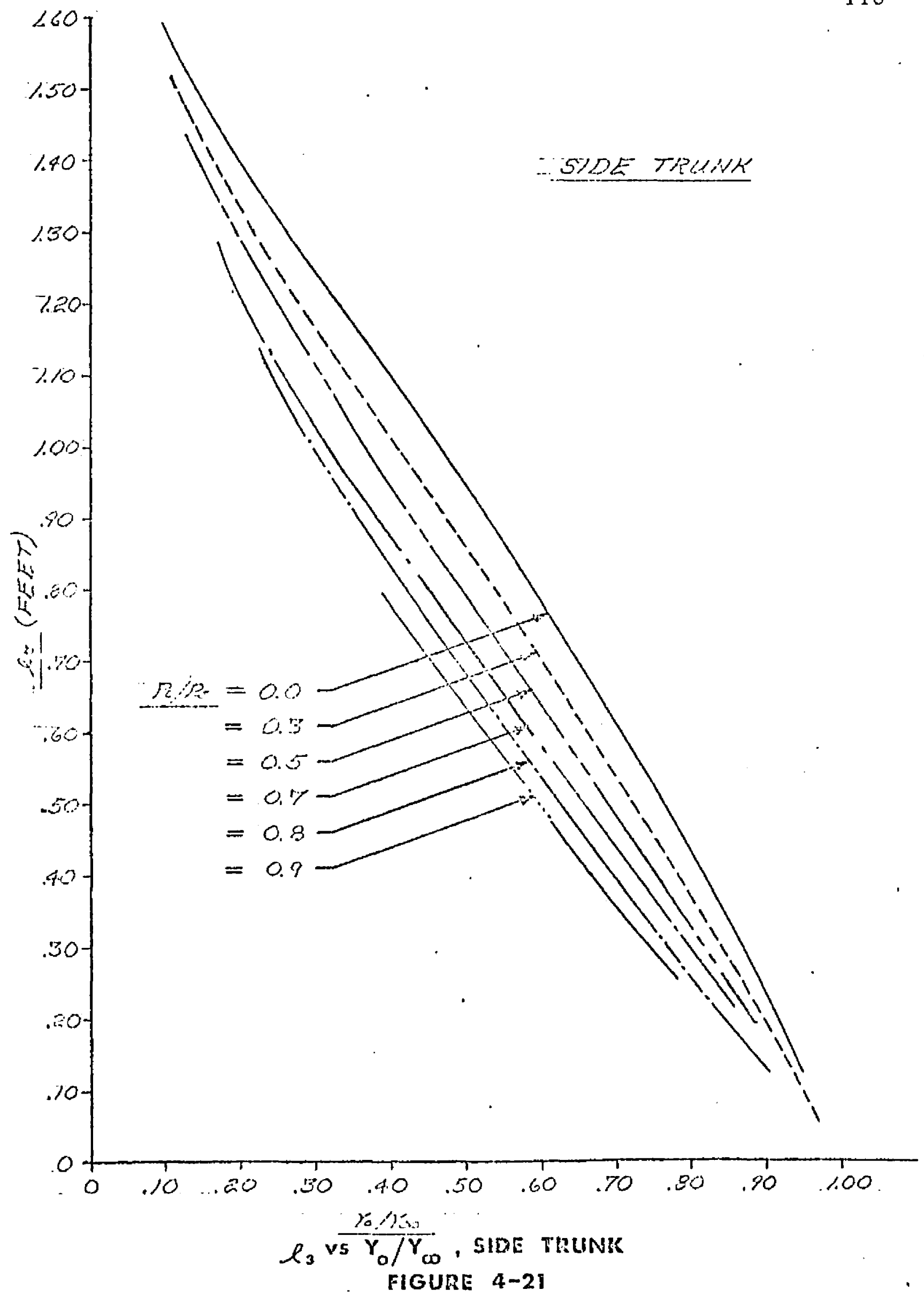




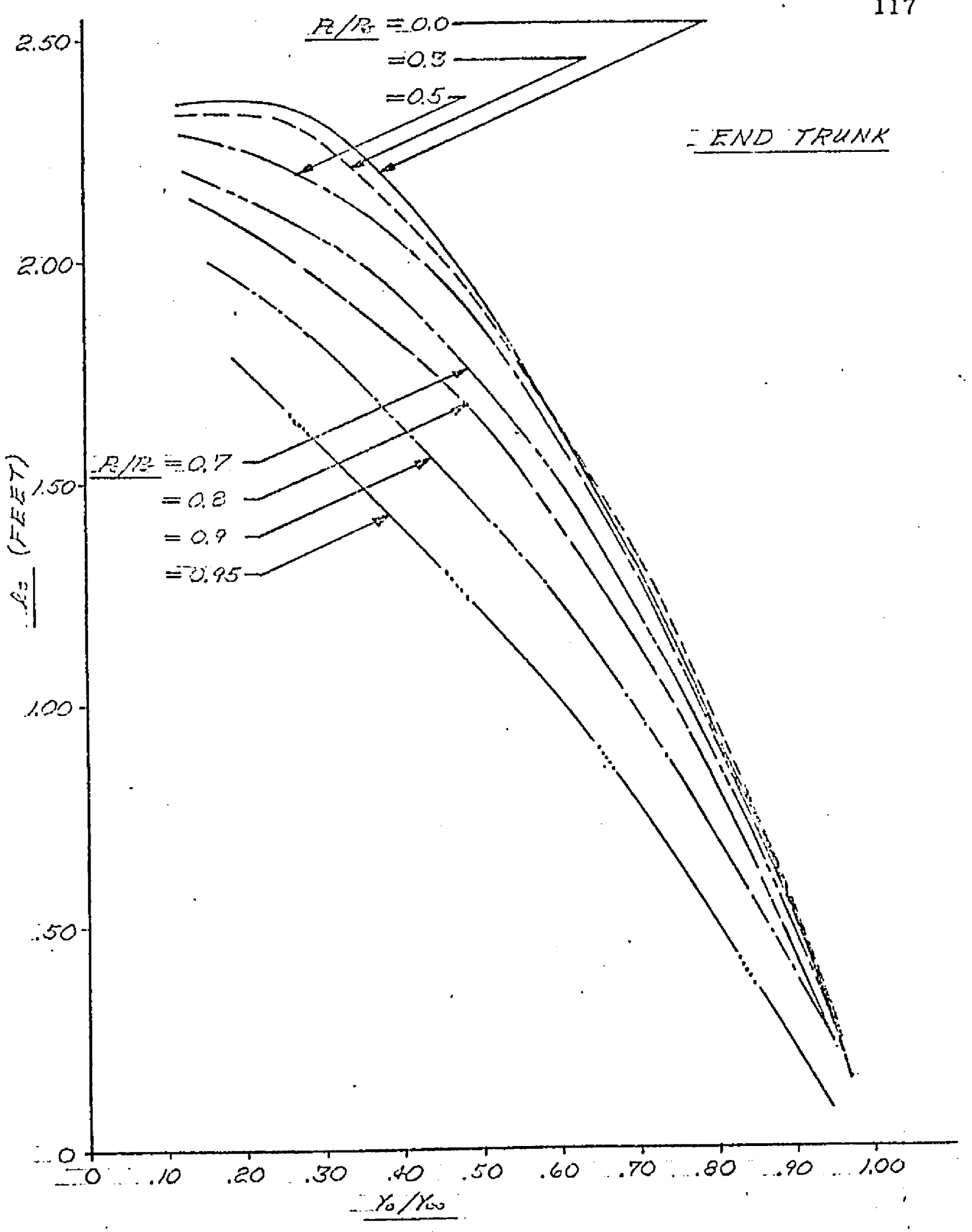

$\ell_{3} v s Y_{0} / Y_{\infty}$, END - TRUNK

FIGURE 4-22. 


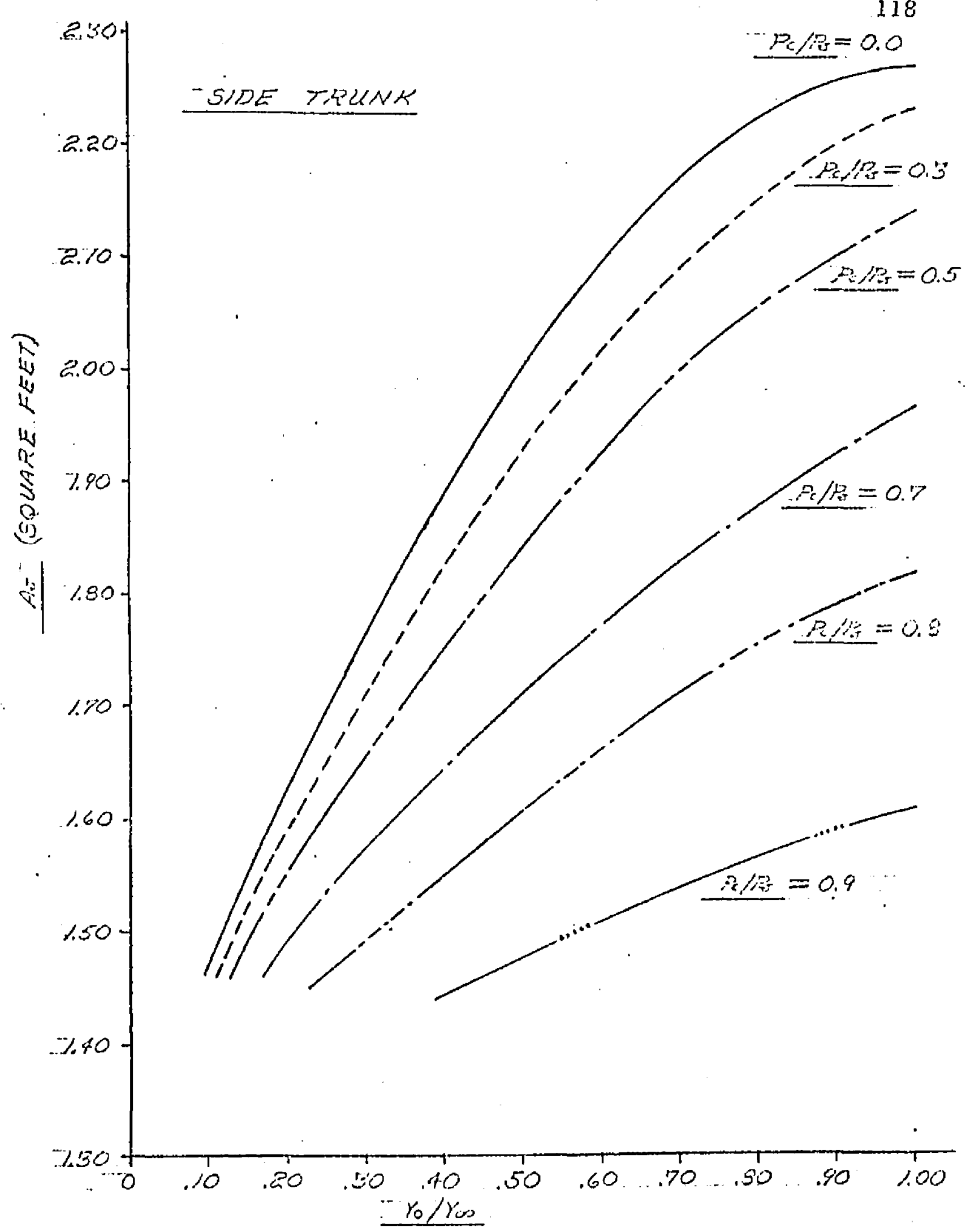

A, vs $Y_{0} / Y_{\infty}$, SIDE TRUNK

FIGURE 4-23 


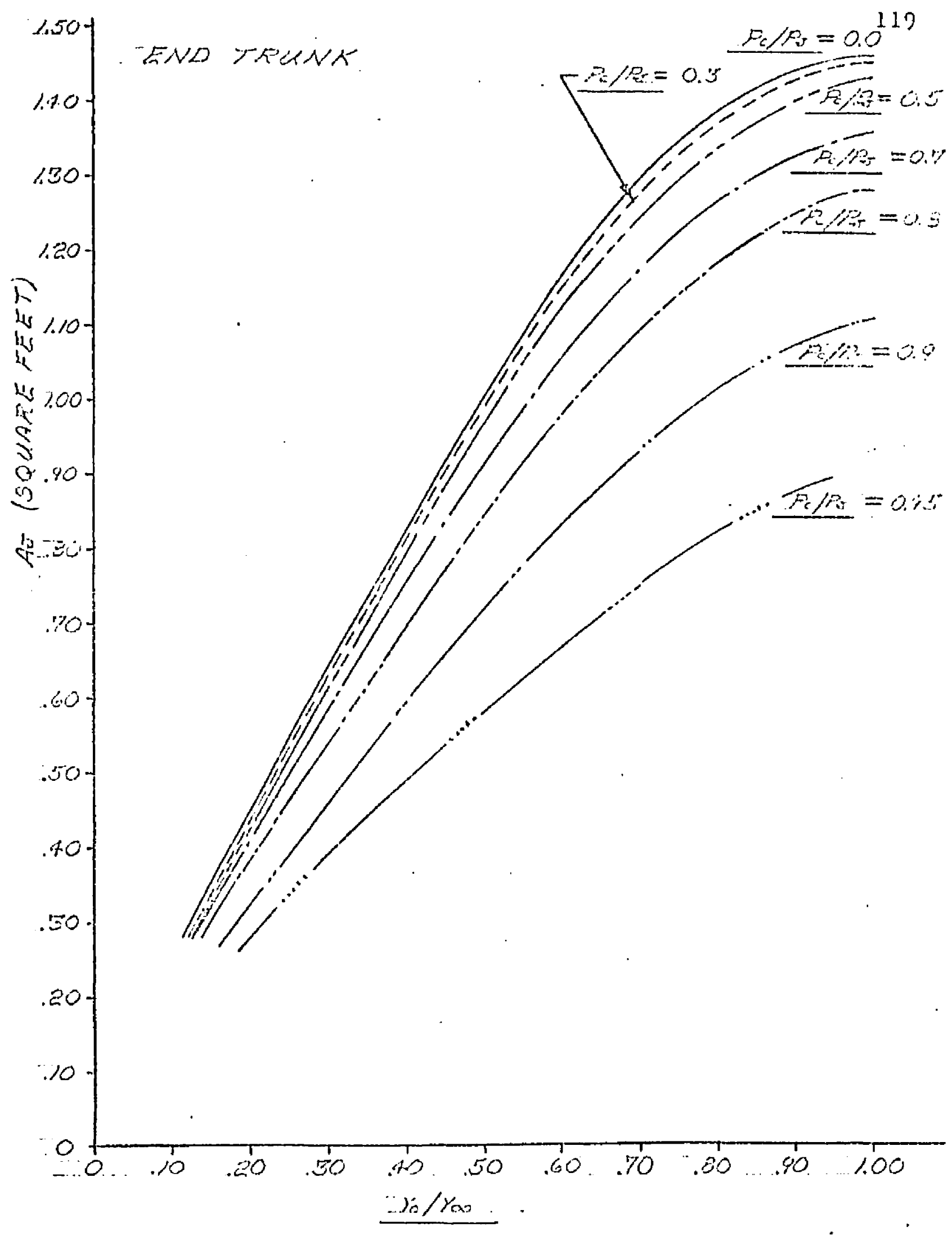

A, vs $Y_{0} / Y_{\infty}$, END TRUNK

FIgURE 4-24 


\section{ANALYSIS OF DISTRIBUTED JET FLOW}

\section{$5.1 \quad$ Introduction}

The Air Cushion Landing System introduces air throughout a large area of the bottom of the trunk in order to provide "air lubrication" to the trunk. This "air lubrication" is necessary to prevent excessive wear of the trunk during takeoff rotation and landing flare. During these maneuvers, the cushion pressure approaches atmospheric pressure and the trunk must carry a portion of the load.

For the ACLS, the peripheral jets are formed by a large number of slots or holes which are distributed over the bottom of the trunk. The Air Cushion Vehicle, on the other hand, normally employs a one continuous nozzle which concentrates the single jet at the point of minimum daylight clearance. Because of these differences, modifications of the concentrated jet theories are necessary when applying them to the distributed jet system.

In this Chapter, modifications to the concentrated jet theories presented in Chapter 2 have been developed. These modifications allow the concentrated jet theories to more closely conform to the actual Air Cushion Landing System distributed jet design.

Two cases have been considered: The Distributed Jet Momentum Theory and the Flow Restrictor Theory.

The Distributed Jet Momentum Theory applies the momentum theories developed in Chapter 2 to a number of jets in series. This theory assumes the cushion pressure is maintained by the change in momentum of the peripheral jet. The momentum theory is developed in Section 5.2.

The Flow Restrictor Theory applies the plenum theory to the trunk configuration for the Air Cushion Landing System. This theory assumes the cushion pressure is maintained by a flow restriction at the cushion periphery. The Flow Restrictor Theory is developed in Section 5.3. 
The symbols are as follows:

\begin{tabular}{|c|c|}
\hline a & $x$ coordinate of the upper trunk attachment point, $\mathrm{ft}$ \\
\hline$a_{j}$ & total area of the orifices in the trunk, $\mathrm{ft}^{2}$ \\
\hline$a_{n}$ & total area of the orifices in the $\mathrm{n}^{\text {th }}$ row, $\mathrm{ft}^{2}$ \\
\hline b & y coordinate of upper trunk attachment point, $\mathrm{ft}$ \\
\hline$c_{D}$ & cushion exhaust nozzle shape coefficient \\
\hline$c_{d}$ & coefficient of discharge for plenum chamber \\
\hline$\left(C_{Q}\right)_{n}$ & flow coefficient for pressure distribution at the $\mathrm{n}^{\text {th }}$ \\
\hline & row of trunk orifices \\
\hline $\mathrm{c}_{\mathrm{T}}$ & effective flow area reduction in the cushion exhaust nozzle \\
\hline & caused by the flow from the trunk orifices \\
\hline$\left(C_{x}\right)_{n}$ & coefficient of discharge for the $\mathrm{n}^{\text {th }}$ row of trunk orifices \\
\hline $\mathrm{D}_{\mathrm{q}}$ & trunk orifice diameter, $\mathrm{ft}$ \\
\hline d & jet height or trunk daylight clearance, $\mathrm{ft}$ \\
\hline$d_{n}$ & jet height for the $\mathrm{n}^{\text {th }}$ row of trunk nozzles, $\mathrm{ft}$ \\
\hline$g_{0}$ & gravitational constant, $\mathrm{ft} / \mathrm{sec}^{2}$ \\
\hline$H$ & $\begin{array}{l}\text { aircraft clearance, the distance between the aircraft hard } \\
\text { structure and the ground, } \mathrm{ft}\end{array}$ \\
\hline$J_{n}^{\prime}$ & the total, reaction from the $n^{\text {th }}$ row of jet orifices, lbs \\
\hline$\ell_{1}$ & partial trunk length (see Figure 5-3), $\mathrm{ft}$ \\
\hline$\ell_{2}$ & partial trunk length (see Figure $5-3$ ), $\mathrm{ft}$ \\
\hline$l_{3}$ & trunk footprint length (see Figure $5-3$ ), $\mathrm{ft}$ \\
\hline N & number of jet orifices per row \\
\hline$n^{\prime}$ & effective number of rows of jets which contribute to cushion \\
\hline & nozzle area flow reduction \\
\hline a & atmospheric pressure, psf \\
\hline & cushion pressure, psf \\
\hline
\end{tabular}




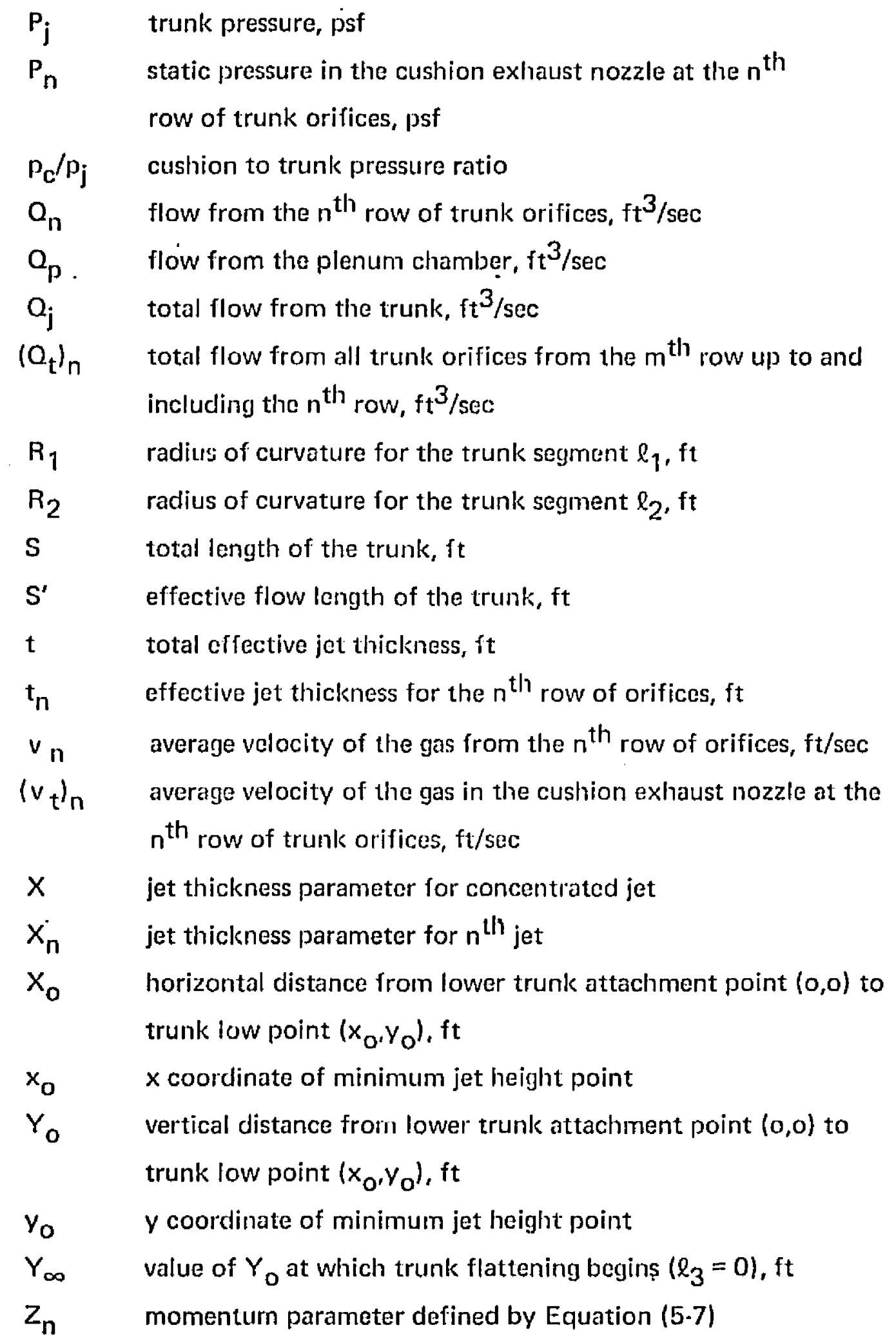




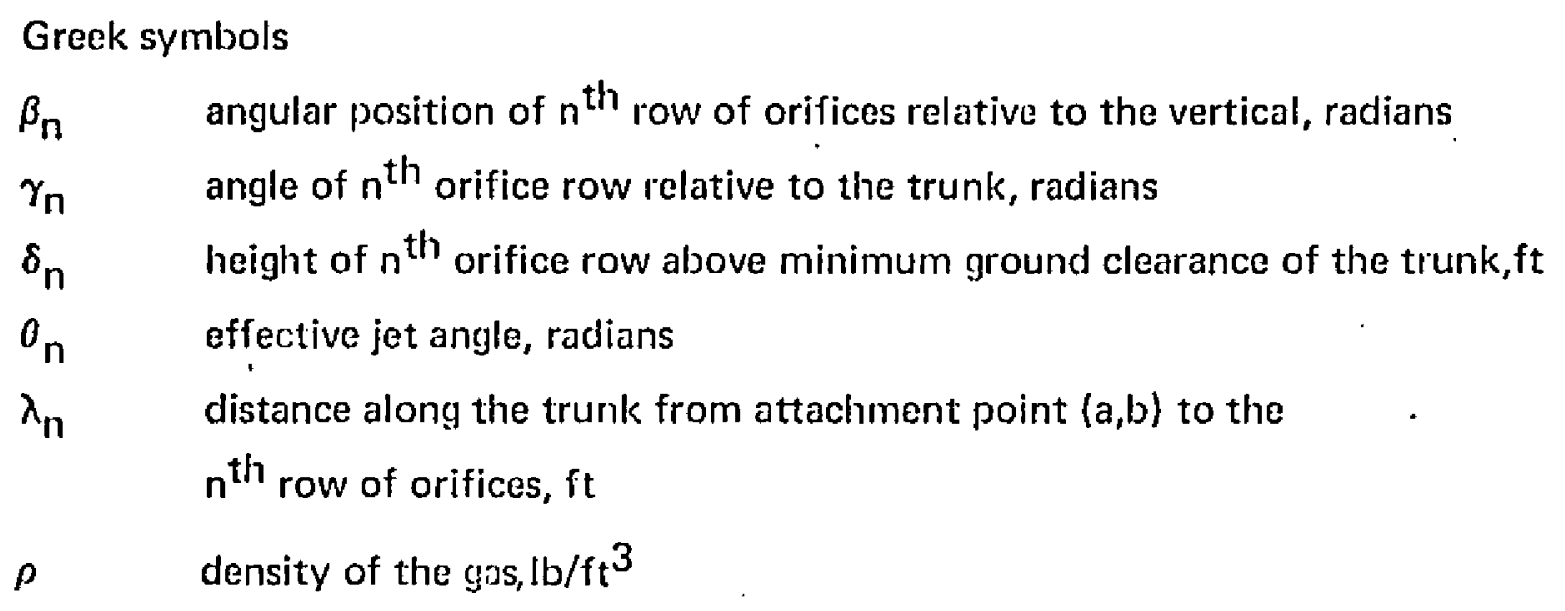

Subscripts

\& first row of orifices inside the cushion

m last row of orifices inside the cushion

n arbitrary row of orifices

5.2 Distributed Jet Momentum Theory

\subsubsection{Approach and Assumptions}

In Chapter 2, several theories for predicting the performance of a periphera! jet air cushion were develojied. These theories assumed that the peripheral jet was formed by a single concentrated silot or nozzle anound the periphery of the cushion. The nozzle configuration for thr: Air Cushion Landing System may be considerably different from the assumed concentrated jet. In particular, the ACLS utilizes a large number of slots or nozzles distributed over the bottom portion of the trunk. Consequently, it was desirable to modify 
TABLE $5-1$

Values of Trunk Design Variables

\begin{tabular}{|l|c|c|}
\hline \multicolumn{1}{|c|}{ VARIABLE } & SYMBOL & VALUE \\
\hline Trunk I.ength & 1 & $4.803 \mathrm{ft}$. \\
Trunk Width & s & $2.667 \mathrm{ft}$. \\
Equivalent jet thickness & $t$ & $: 03832 \mathrm{ft}$. \\
Upper trunk atlachment & a & $1.44 \mathrm{ft}$. \\
Lower trunk attachment & b & $1.00 \mathrm{ft}$. \\
Number of rows of orifices & M & 8. \\
Diameter of orifices & D & $.026 \mathrm{ft}$ \\
Total number of orifices & -- & 192 \\
Porosity & $\xi$ & .049 \\
\hline
\end{tabular}

\begin{tabular}{|c|c|c|c|}
\hline \multicolumn{4}{|c|}{ ORIFICE DETAILS } \\
\hline $\begin{array}{c}\text { KOW } \\
\text { NUMBER } \\
\text { n }\end{array}$ & $\begin{array}{c}\text { ROW } \\
\text { DISTANCE } \\
\lambda{ }_{\mathrm{ft} .}\end{array}$ & $\begin{array}{c}\text { JE'T } \\
\text { THICKNESS } \\
t \\
\text { ft. }\end{array}$ & $\begin{array}{c}\text { JE'T } \\
\text { ANGLE } \\
\gamma_{n} \\
\text { Radians }\end{array}$ \\
\hline 1 & 2. 599 & .00479 & 0 \\
\hline 2 & 2.703 & .00479 & 0 . \\
\hline 3 & 2. 807 & .00479 & 0 \\
\hline 4 & 2.912 & .00479 & 0 \\
\hline 5 & 3.016 & .00479 & 0 \\
\hline 6 & 3.120 & .00479 & 0 \\
\hline 7 & 3.224 & .00479 & 0 \\
\hline 8 & 3.328 & .00479 & 0 \\
\hline
\end{tabular}


the theories to more closely approximate the ACLS configuration. In this section, the jet configuration was assumed to be represented by a series of continuous slots along the bottom portion of the trunk. This configuration is illustrated in Figure 5-1.

The general approach to the problem was to assume a trunk clearance (d) for given values of trunk pressure $\left(\mathrm{p}_{\mathrm{j}}\right)$ and recovery pressure ratio $\left(\mathrm{p}_{\mathrm{c}} / \mathrm{p}_{\mathrm{j}}\right)$. The jet height for each of the trunk nozzles was determined from the trunk sluape programs developed in Chapter 4. Starting on the atmospheric side of the trunk, the pressure increment across each jet was calculated in succession until the pressure in the cushion was determined. If the calculated and assumed value of cushion pressure rlid not agree, the jet height was adjusted until agreement was achieved.

The pressure increment across each jet is dependent upon the momentum theory assumed. However, when the pressure increment is small, alf of the momentum theories developed in Chapter 2 give similar results. In view of the small pressure increments associated with a series of distributed jets, only two theories - the thin jet theory and the exponential theory - were selected for further development.

The development of the distributcd jet momentum theory is similar to the concentrated jet theories presented in Chapter 2. The assumptions made in Section 2.4 apply to the distributed jet system. Moreover, the assumptions made in Sections 2.5 and 2.6 are applicable when the thin jet or the exponential theorias are applied to the distributed jet system. Two additional assumptions are necessary. These assumptions are as follows.

5.2.1.1 The jets are formed by a series of continuous slots along the bottom of the trunk.

5.2.1.2 The flow from any given jet is related only to the static pressure difference across the nozzle. The effect of flow from other jets is neglected. 


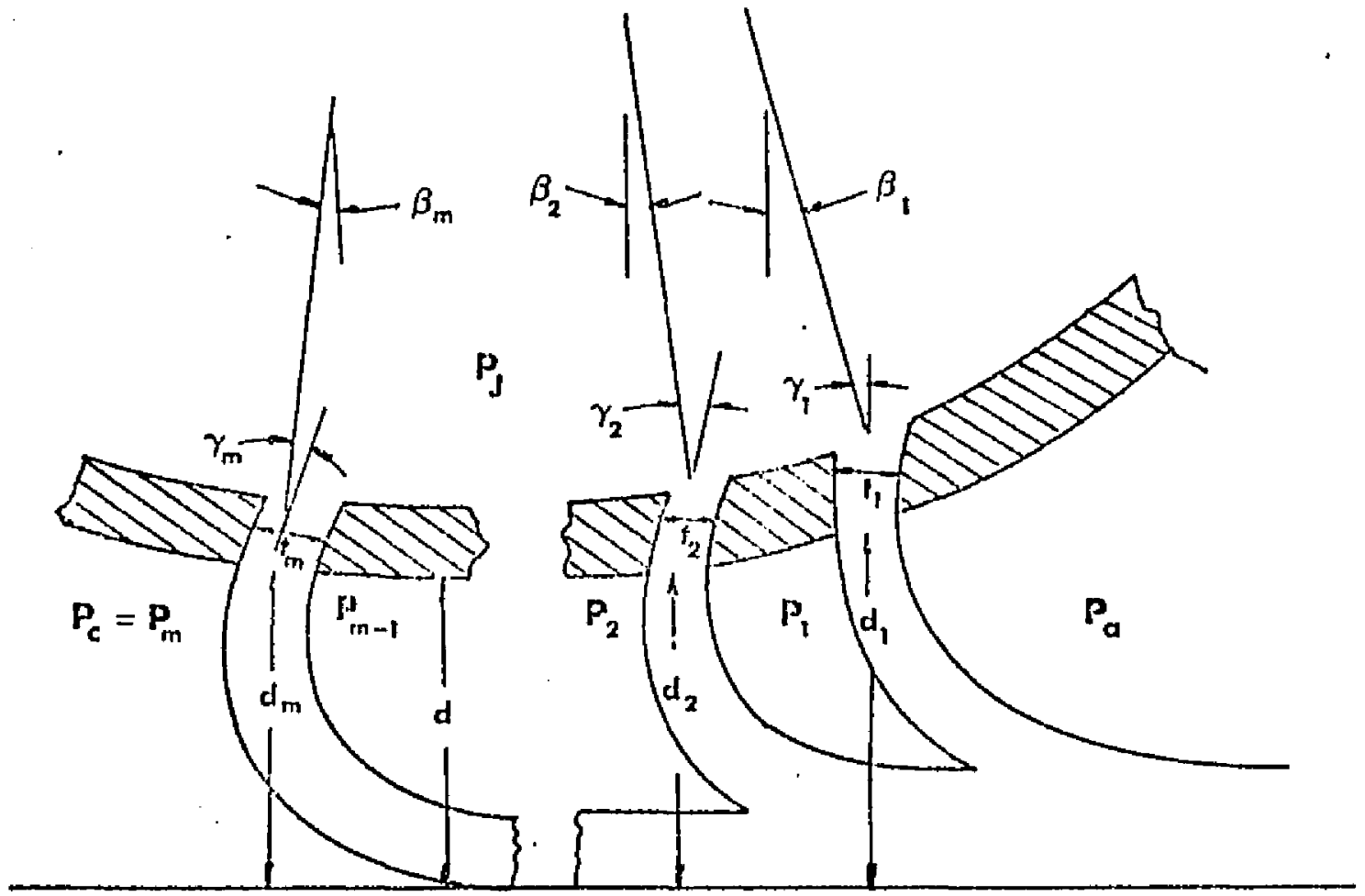

\section{DISTAIBUTED JET GEOMETRY}

FIGURE $5-1$ 


\subsubsection{Force Equilibrium Across the Jets}

In Sections 2.4.6, 2.5.6, and 2.6.6 force equilibrium was applied in the $x$ direction to a control volume containing the peripheral jet. The resulting expression equated the product of the jet height and the pressure increment across the jet to the change of momentum of the jet in the $x$ direction. A similar expression may be developed for each jet in the series shown in rigure 5-1.

Force equilibrium applied to the first jet in Figure 5-1 gives:

$$
d_{1}\left(P_{1}-P_{a}\right)=\frac{J_{1}^{\prime}}{s}\left(1+\sin O_{1}\right)
$$

where $0_{1}=\gamma_{1}+\phi_{1}$

Similarly, force equilibrim applied to the second jet is:

$$
d_{2}\left(P_{2}-P_{1}\right)=\frac{J_{2}^{\prime}}{s}\left(1+\sin \theta_{2}\right)
$$

Across the $n^{\text {th }}$ jet, force equilibrim gives:

$$
d_{n}\left(P_{n}-P_{n-1}\right)=\frac{J_{n}^{\prime}}{s}\left(1+\sin \theta_{n}\right)
$$

where $\theta_{n}=\gamma_{n}+\phi_{n}$

In general, the pressure at any point $P_{n}$ may be found by rearranging Equation (5-1c).

$$
P_{n}=\frac{J_{n}^{\prime}}{d_{n} s}\left(1+\sin 0_{n}\right)+P_{n-1}
$$


The value of $J_{n}^{\prime}$ is dependent upon the flow theory selected. The simple jet theory and the exponential theory are considered most appropriate for the distributed jet case. Both of these theories are applicable to thin jets, and the distributed jet configuration involves a series of thin jets.

The expressions for $\mathrm{J}_{\mathrm{n}}^{\prime}$ given by the two thin jet theories were developed in Sections 2.5 and 2.6 respectively. When applied to the $n^{\text {th }}$ jet in the series, the momenturn expressions become:

Thin jet theory

$$
J_{n}^{\prime}=2 S t_{n}\left(P_{j}-P_{n-1}\right)
$$

Exponential theory

$$
J_{n}^{\prime}=2 S t_{n}\left(P_{j}-P_{n-1}\right)\left[\frac{1}{2 X_{n}}\left(1-e^{\left.-2 X_{n}\right)}\right]\right.
$$

where $x_{n}=\frac{t_{n}}{d_{n}}\left(1+\sin 0_{n}\right)$

The momentum expressions, Equations $(5-3 a)$ for $(5-3 b)$, may now be combined with Equations (5-2) and (5-4) to provide an expression for the pressure across the $n^{\text {th }}$ jet. The results are:

Thin jet theory

$$
P_{n}=2\left(P_{j}-P_{n-1}\right) X_{n}+P_{n-1}
$$

Exponential theory

$$
P_{n}=\left(P_{j}-P_{n-1}\right)\left(1-e^{-2 X_{n}}\right)+P_{n-1}
$$


A general expression for the pressure across the jet may be written as follows:

$$
P_{n}=2\left(P_{j}-P_{n-1}\right) Z_{n}+P_{n-1}
$$

where:

$$
\begin{aligned}
& \text { for thin jet theory } \\
& z_{n}=x_{n}
\end{aligned}
$$

for exponential theory

$$
z_{n}=\frac{1}{2}\left(1-e^{-2 x_{n}}\right)
$$

\subsubsection{Geometric Compatibility}

In order to determine $x_{n}$ for each of the jets, it is necessary to determine the trunk shape and the location of each jet. This problem may be solved by using the trunk shape solutions given in Sections 4.4 or 4.5 . For a given $a, b, \ell$ and $p_{c} / p_{j}$, the trunk shape may be determined by the method derived in Section 4.4. It is necessary, in addition, to specify the location of the jets and their angle relative to the trunk membrane. These two variables are specified by $\gamma_{n}$ and $\lambda_{n}$ which are clefined geometrically in Figures 5-1 and 5.2 respectively.

The trunk shape analysis presented in Section 4.4 predicts the lowest point on the trunk $\left(x_{0}, y_{0}\right)$. This is the coordinate point at which the minimum jet height (trunk clearance) is measured. This height is specified as $d$ and is shown in Figure 5-2. All other jet heights may be measured relative to the minimum $d$ in terms of $\delta_{n}$ as shown in Figure 5-2. Consequently, it is possible to write the jet height of any nozzle as:

$$
d_{n}=d+\delta_{n}
$$




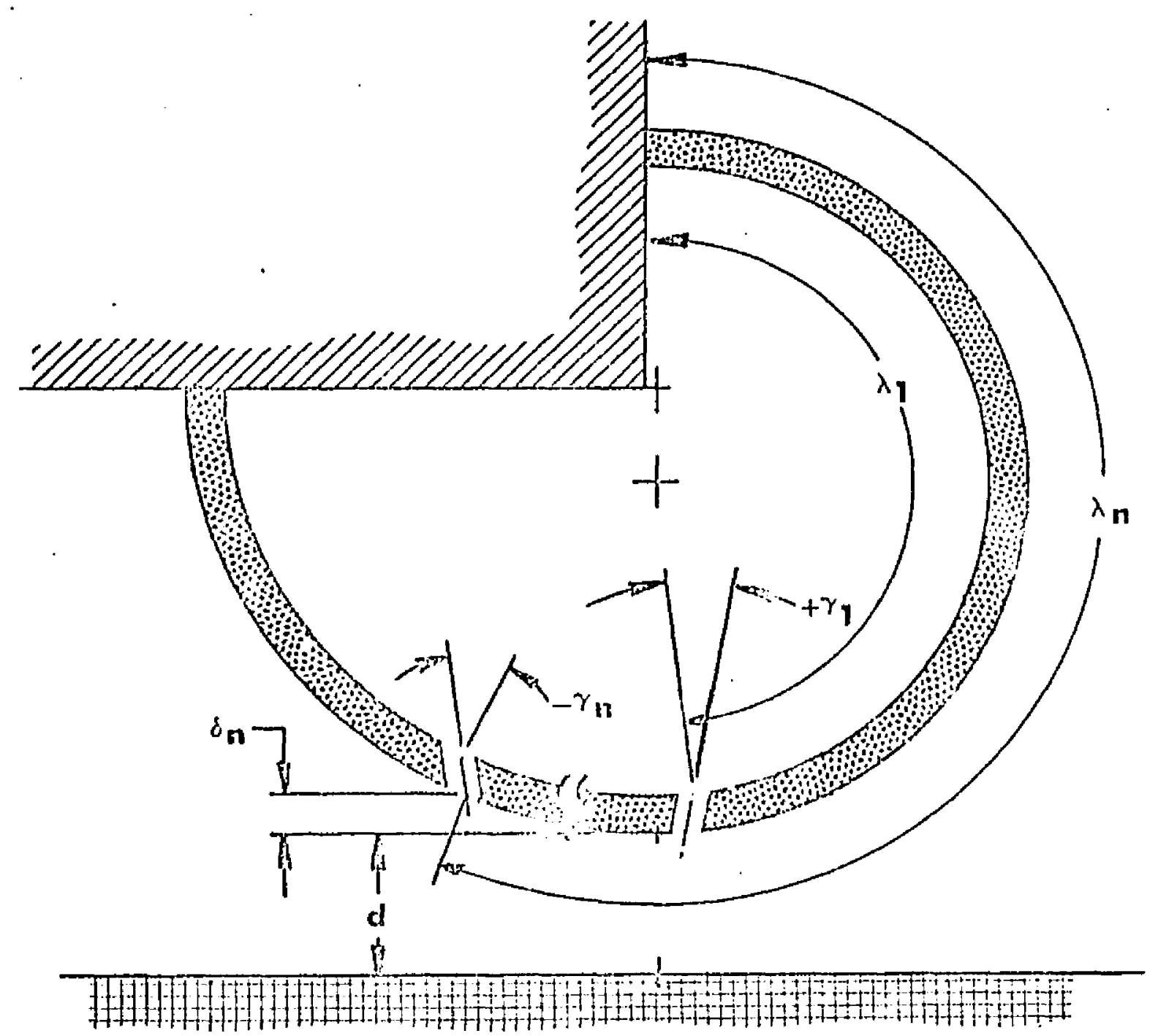

TRUNK GEOMETAY FOR DISTRLBUTED JET

FIGURE 5-2 
It is now possible to calculate $\delta_{\mathrm{n}}$ and $O_{\mathrm{n}}$ from trunk geometry. These values, in turn, allow the calculation of $x_{n}$ and $P_{n}$.

It is possible for the $n^{\text {th }}$ jet nozzle to be located on any one of the three trunk segments shown in Figure 5.3. Each of these locations constitutes a different case. The three cases are listed as follows:

\section{Case 1}

The $\mathrm{n}^{\text {th }}$ jet is on the atmospheric side of the low point.

\section{Case 2}

The $\mathrm{n}^{\text {th }}$ jet is at the low point.

\section{Case 3}

The $\mathrm{n}^{\text {th }}$ jet is on the cushion side of the low point.

Case 1 may be recognized by the following condition:

$$
\ell_{1}-\lambda_{n}>0
$$

For Case 1 , the remaining geometric relationships may be derived from the geometry shown in Figure 5-3(a). These relationships are:

$$
\begin{aligned}
& \beta_{n}=\frac{\ell_{1}-\lambda_{1}}{R_{1}} \\
& \delta_{n}=R_{1}\left(1-\cos \beta_{n}\right) \\
& 0_{n}=-\beta_{n}+\gamma_{n}
\end{aligned}
$$


Case 2 may be recognized by the following condition:

$$
\ell_{1}+\ell_{3}-\lambda_{n} \geqslant 0 \geqslant \ell_{1}-\lambda_{n}
$$

The remaining geometric relationships as shown in Figure $5 \cdot 3(b)$ are:

$$
\begin{aligned}
& \beta_{n}=0 \\
& \delta_{n}=0 \\
& o_{n}=\gamma_{n}
\end{aligned}
$$

Case 3 may be recognized by the following condition:

$$
\ell_{1}+\ell_{3}-\lambda_{n}<0
$$

The remaining geometric relationships as shown in Figure 5-3(c) are:

$$
\begin{aligned}
& \beta_{n}=\frac{\lambda_{n}-\ell_{1}-\ell_{3}}{R_{2}} \\
& \delta_{n}=R_{2}\left(1-\cos \beta_{n}\right) \\
& \theta_{n}=\beta_{n}+\gamma_{n}
\end{aligned}
$$

\subsubsection{Solution of Equations}

The distributed jet momentum theory may now be solved on an iterative basis as follows. 

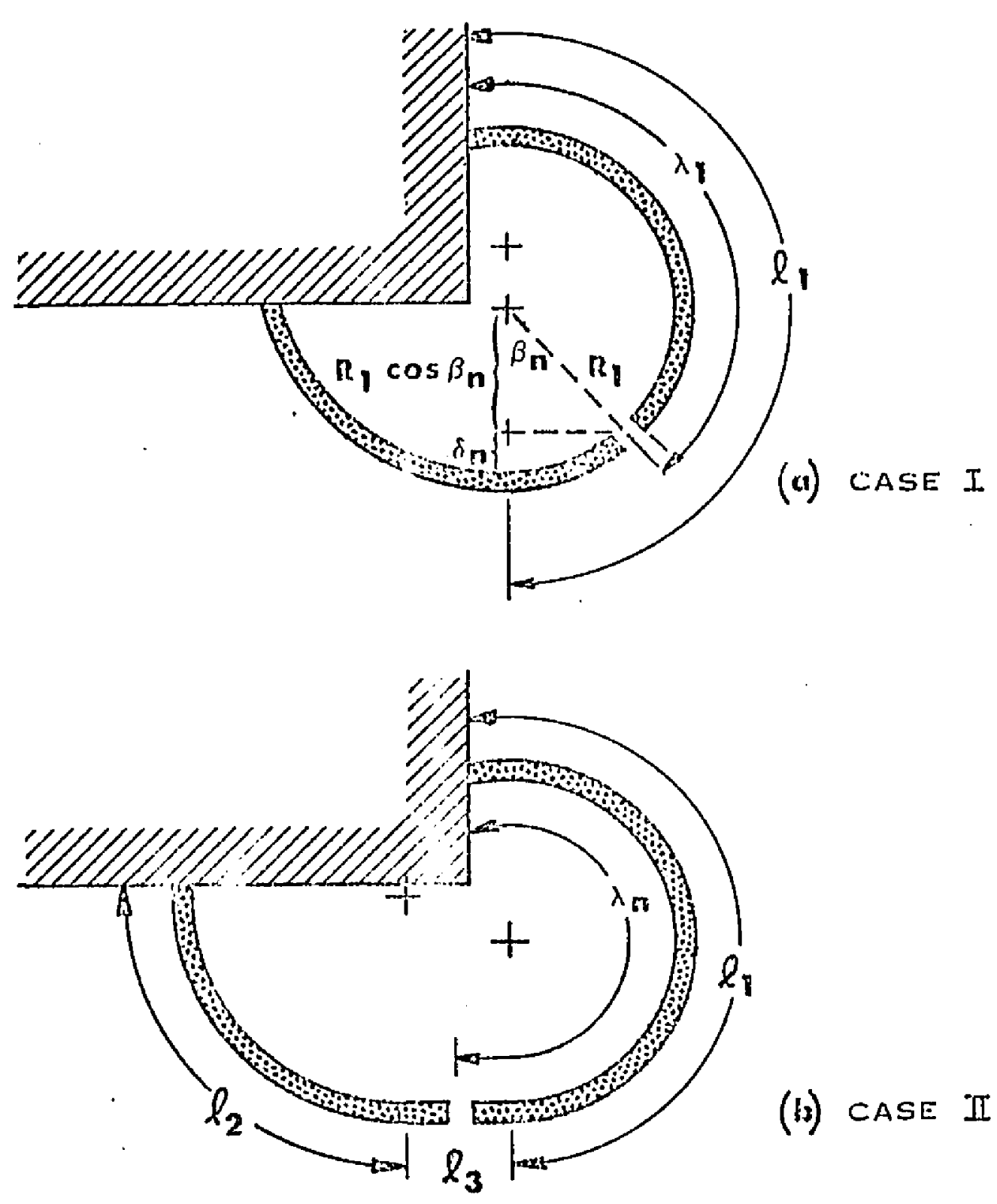

(b) CASE II

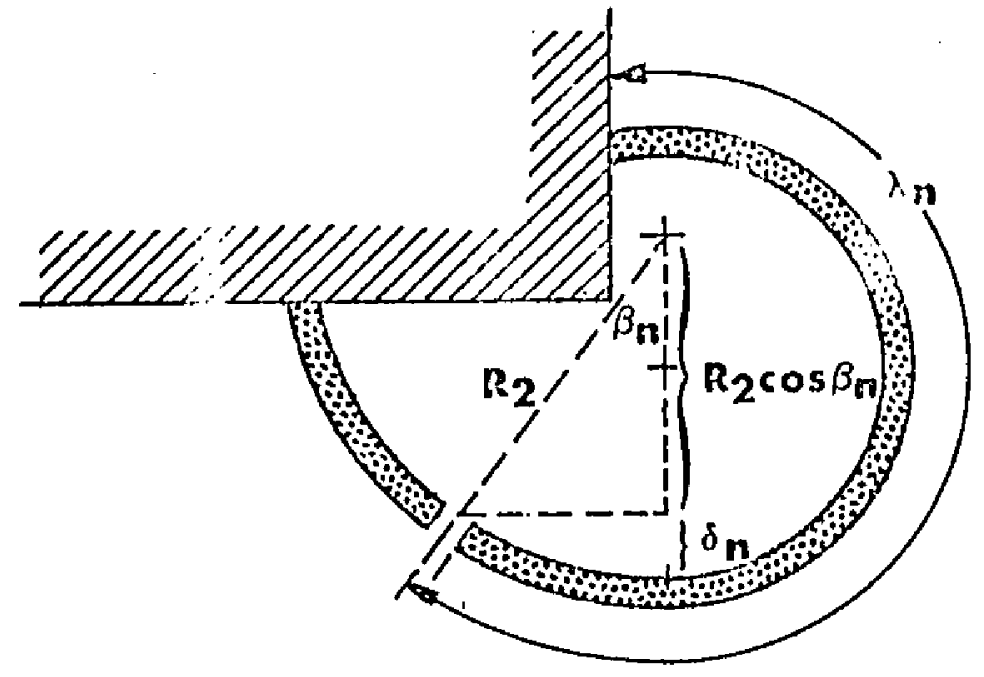

(c) CASE III

THREE CASES FOR JET LOCATIONS FIGURE $5-3$ 
(1) Given $a, b, \ell$ and $p_{c} / p_{j}$ the trunk shape may be found using the procedure of Sections 4.4 or 4.5 . This procedure gives $R_{1}, R_{2}, \ell_{1}, \ell_{2}$, and $l_{3}$

(2) Assume a maximum value of $d$. This value may be determined from the simple jot pressure relationship given in Section 2.511 .

$\mathrm{P}_{\mathrm{c}} / \mathrm{P}_{\mathrm{j}}=2 \mathrm{X}=\frac{2 \mathrm{t}}{\mathrm{d}}(1+\sin 0)$

Rearranging:

$d=\frac{2 \mathrm{t}(1+\sin 0)}{\mathrm{p}_{\mathrm{c}} / \mathrm{p}_{\mathrm{j}}}$

and

$d_{\max }=\frac{4 t}{p_{c} / p_{j}}$

(3) The other known variables are:

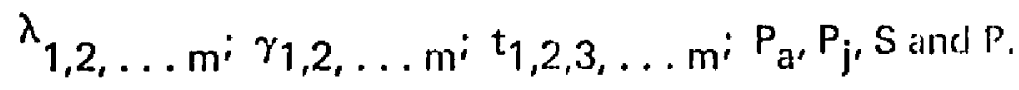

With the assumed value of $d$, it is possible to calculate $X_{n}$ from Equation (5-4). Equations (5-8), (5-11), and (5-12) provide the values of $d_{n}$ and $O_{n}$ which are required in the calculation of $x_{n}$. 
(4) It is now possible to solve for the pressure distribution across the jets. This solution is achieved by applying Equation (5-6) to each jet in turn, starting at the first jet (Figure 5-2) and proceeding inward.

$$
\begin{gathered}
P_{1}=2\left(P_{j}-P_{0}\right) Z_{1}+P_{a} \\
P_{2}=2\left(P_{j}-P_{1}\right) Z_{2}+P_{1} \\
\cdot \quad \cdot \cdot \cdot \cdot \cdot \cdot \\
\cdot \cdot \cdot \cdot \cdot \cdot \\
P_{m}=2\left(P_{j}-P_{m-1}\right) Z_{m}+P_{m-1}
\end{gathered}
$$

(5) The : umed value of $d$ is correct when

$$
\frac{P_{m}-a}{P_{j}-i_{a}}=p_{c} / p_{j}
$$

If $\mathrm{p}_{c} / \mathrm{p}_{\mathrm{j}}$ is greater than $\left(\mathrm{P}_{\mathrm{m}}-\mathrm{P}_{\mathrm{a}}\right) /\left(\mathrm{P}_{\mathrm{j}}-\mathrm{P}_{\mathrm{a}}\right)$, decrease $\mathrm{d}$ and repeat the procedure until agreement is reached.

(6) Once Equation $(5-14)$ is satisfied, it is possible to calculate the flow. The flow equations developed in Sections 2.5 and 2.6 applied to each jet give the following relationship:

$$
\mathrm{Q}_{j}=\sum_{n=T}^{m} S t_{n} \sqrt{\frac{2 g_{0}}{\rho}\left(P_{j}-P_{n-1}\right)}\left(C_{Q}\right)_{n}
$$


where:

$$
\begin{aligned}
& \left(c_{Q}\right)_{n}=1 \text { for thin jet theory } \\
& \left(C_{Q}\right)_{n}=\left[\frac{1}{x_{11}}\left(1-e^{\left.-x_{n}\right)}\right] \quad\right. \text { for exponential theory }
\end{aligned}
$$

\subsection{Flow Restricior Theory}

\subsubsection{Approach and Assumptions}

The general configuration of distributed jets is shown in Figure 5-1. In the flow restrictor theory, it is assumed that the jets are formed by rows of circular holes rather than by continuous slots. As a result of the spacing between the holes, passages for air flow from the cushion exist. A continuous momentum seal does not exist, and the flow may approach that of a plinum chamber. The plenum chamber assumptions developed in Section 2.9 are applicable to this case. The additional assumptions for this case are as follows:

5.3.1.1 The lowest point of the trunk is specified by $\left(x_{0}, y_{0}\right)$. The distance between $\left(x_{0}, y_{0}\right)$ and the ground is $d$, the minimum jet height (trunk clearance).

5.3.1.2 The jets on the cushion side of $\left(x_{0}, y_{0}\right)$ supply all the flow into the cushion which maint:ins the height $d$. The momentum seal effect of these jets is neglected.

5.3.1.3 The jets on the outside of $\left(x_{0}, y_{0}\right)$ act to reduce the flow area. A flow coefficient $\left(\mathrm{C}_{\mathrm{T}}\right)$ is used to account for this area reduction. 
5.3.1.4 The flow from the cushion is dependent on the shape of the cushion exhaust nozzle (which is formed b.tween the trunk and the ground). A flow coefficient $\left(C_{D}\right)$ is $t ! \cdot($ to accutunt for this effect.

The jet height (trunk clearance) may be estimated by assuming that the pressure on the cushion side of $\left(x_{0}, y_{0}\right)$ is uniform und equal to the cushion pressure. The trunk pressure is known. Since the total orifice area on the custiion side of $\left(x_{0}, v_{0}\right)$ is also known, the flow into the cusinion may be calculated. Assuming the plenum theory is applicable, the jet height will rise until the flow from the plenum cquals the flow into the plenun. The jet height may be determined by finding the value of $d$ whicl: equates the flow out to the flow in. The expression for flow from the plenum is developed in Section 5.3.2. The flow to the plenum is developed in Section 5.3.3. The jet height is then determined in Section 5.3.4.

A more exact determination of flow and jot height based upon a sequential anilysis of the flow and pressure increment associated with each row of orificcs is presented in Section 5.3.5.

\subsubsection{Determination of Flow from Plonum}

It was shown in Section 2.8 that the flow from a plenum chamber is given by:

$$
\dot{Q}_{p}=\sqrt{\frac{29_{o}}{\rho}\left(P_{c}-P_{a}\right)} s d C_{d}
$$

The coefficient of discharge $C_{d}$ is dependent upon a large number of variables. For the purpose of this analysis, the dependence on nozzle pressure ratio, exhaust nozzle shape and jet configuration will be considered.

The coefficient $C_{d}$ may be considered as the product of two coefficients: 


$$
c_{d}=\left(c_{T}\right)\left(c_{D}\right)
$$

where:

$$
\begin{aligned}
& C_{D}=\text { nozzle shape coefficient } \\
& C_{T}=\text { flow area reduction coefficient }
\end{aligned}
$$

From Figure $5-4$ it is evident that the nozzle slape for the plentum citami if exhanst approaches that of a convergent-djvergent nozzle. Consequently, $\mathrm{C}_{[,}$, should approach the cous isicnt of discharge for a nozzle.

The valus of $\mathrm{C}_{\mathrm{T}}$ is dependent on the flow area reduction caused by the jets outside of point $\left(x_{0}, y_{0}\right)$ (see Assumption :.3.1.3). Figure 5.5 shows a typical orifice pittern. Adjacent rows of orifices are generatly not aligned in the direction of flow. Consequently, the cushion flow must follow circuitous paths between the orifices. As a result, the fective flow arcit is reduced and friction is increased.

The value of $\mathrm{C}_{T}$ may be approximated from an estimate of the effective flow area reduction . . used $b y$ the nozzles. The effective flow area is proportional to the effective flow width:

$$
S^{\prime}=S-(N)\left(D_{q}\right)\left(n^{\prime}\right)
$$

where:

$$
\begin{aligned}
& S^{\prime}=\text { effective flow width } \\
& S=\text { actual flow width } \\
& n^{\prime}=\text { effective number of rows of orifices which contribute to flow area reduction } \\
& D_{q}=\text { diameter of orifices } \\
& N=\text { number of orifices per row } \\
& \text { The coefficient }\left(C_{T}\right) \text { may now be cstimated as follows: }
\end{aligned}
$$

$$
\mathrm{C}_{\mathrm{T}} \approx \frac{\mathrm{S}^{\prime}}{\mathrm{S}}
$$

The actual value of $\mathrm{C}_{T}$ requires experimental determination. 


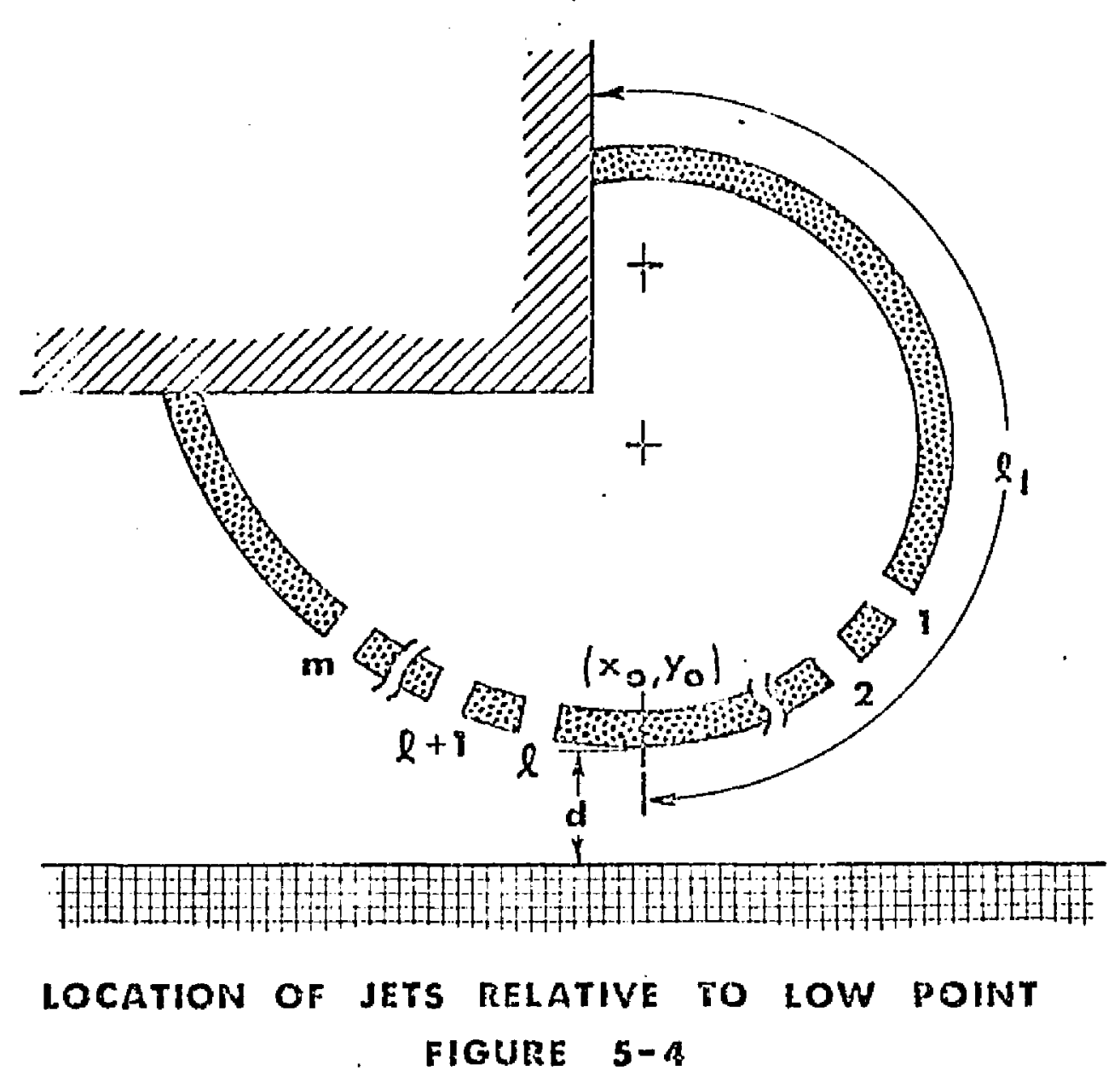




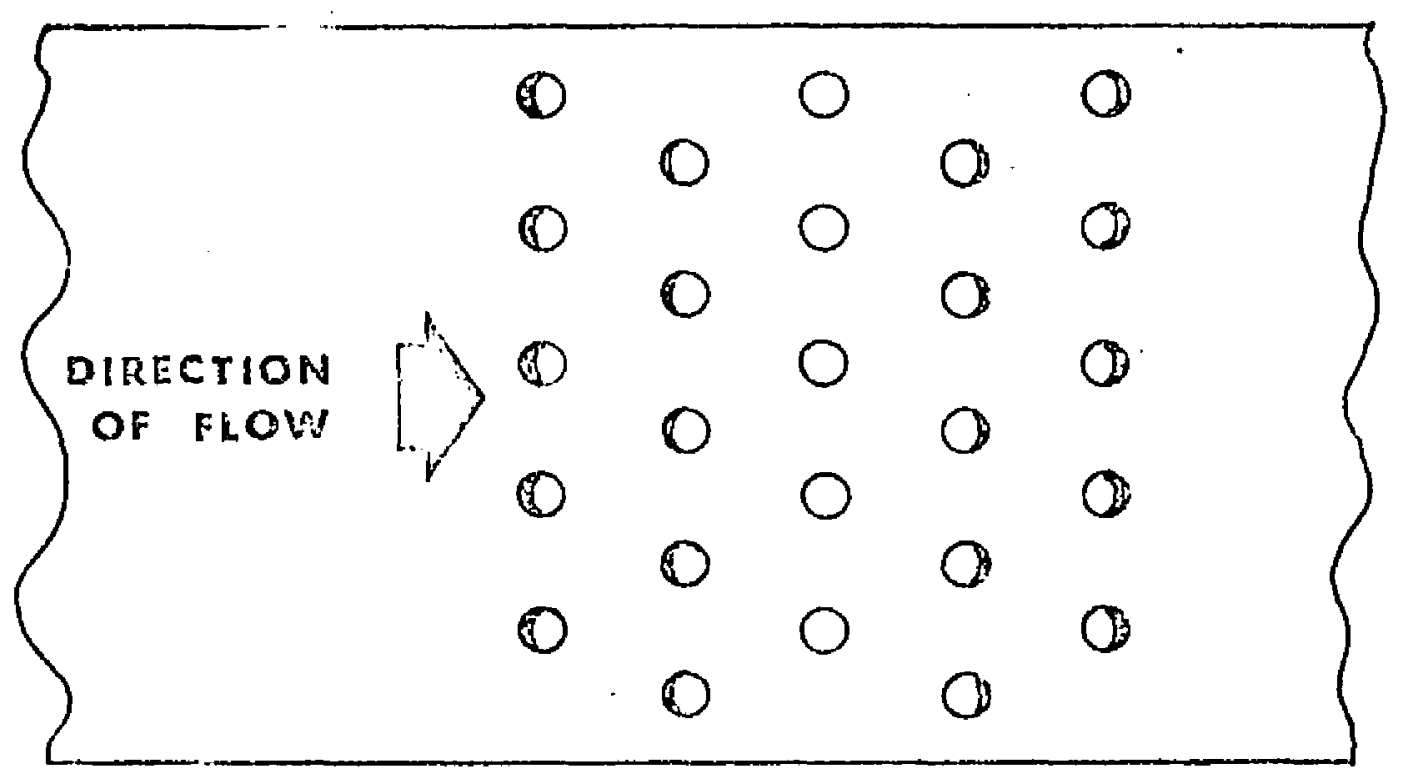

TYPICAL JET SPACING

FIGUIE 5-5 


\subsubsection{Determination of Flow to Plenum}

The flow to the plenum chamber, based on Assumption 5.3.1.1, is the sum of the flow from the jets on the cushion side of point $\left(x_{0}, y_{0}\right)$. The first jet on the cushion side is represented by the $\ell^{\text {th }}$ row in Figure 5.4. The last jet is represented by the $m^{\text {th }}$ row. The flow may be written: .

where:

$$
\mathrm{a}_{\mathrm{P}}=\sum_{n=l}^{m} a_{n} \sqrt{\frac{2 g_{0}}{\rho}\left(P_{j}-P_{n}\right)}\left(C_{x}\right)_{n}
$$

$$
\begin{array}{ll}
a_{p} & =\text { flow to plenum chamber } \\
a_{n} & =\text { area of orifices in } n^{\text {th }} \text { row } \\
P_{n} & =\text { exhaust pressure for holes in } n^{\text {th }} \text { row } \\
\left(C_{x}\right)_{n} & =\text { discharge coefficient for holes in } n^{\text {th }} \text { row }
\end{array}
$$

The total jet flow is:

$$
\mathrm{a}_{j}=\sum_{n=1}^{m} a_{n} \sqrt{\frac{2 g_{o}}{\rho}\left(P_{j}-P_{n}\right)}\left(C_{x}\right)_{n}
$$

The flow may be approximated by letting $P_{n}=P_{c}$ for $n>Q$ and $P_{n}=P_{a}$ for $n \leqslant$ $\ell$.

\subsubsection{Determination of Jet Height}

The jet height may be determined by equating the flow into the plenum, Equation (5-19), to the flow from the plenum, Equation (5-20), and rearranging. The result is:

$$
d=\frac{\sum_{n=\ell}^{m} a_{n} \sqrt{\left(P_{j}-P_{n}\right)}\left(c_{x}\right)_{n}}{s \sqrt{\left(P_{c}-P_{a}\right)}\left(C_{T}\right)\left(C_{D}\right)}
$$


As an approximation, $P_{n}$ can be taken equal to $P_{c}$. The result then becomes:

$$
d \approx \sqrt{\frac{p_{j}}{p_{c}}-1} \frac{\sum_{n=l}^{m} a_{n}}{s} \frac{\left(C_{x}\right)}{\left(C_{T}\right)\left(C_{D}\right)}
$$

Equation (5-22) shows that for the flow restrictor theory, the jet heiglit is dependent upon the ratio of $\bigcap_{\mathrm{c}} / \mathrm{p}_{\mathrm{j}}$. Consequently, the parameter $\mathrm{p}_{\mathrm{c}} / \mathrm{p}_{\mathrm{j}}$ continues to be a valuable dimensionless quantity for relating the independent and dependent variables associated with the system performance.

\subsubsection{Detormination of Pressure Distribution}

A more exact prediction of flow and jet height is dependent upon a more exact prediction of the pressure distribution across the jets. Such a prediction has been developed in this section by a sequential analysis of the flow from each row of orifices. The flow is assumed to be governed by flow restriction as in the plenum theory.

The assumptions associated with the plenum theory (Section 2.9) and the flow restrictor theory (Section 5.3.1) apply to this analysis. In addition, the following assumptions apply.

5.3.5.1 Flow is adiabatic, incompressible and frictionless.

5.3.5.2 Flow from the jets impinges on the ground and is directed in all directions. The total pressure of the plenum exhaust is equal to the static cushion pressure.

5.3.5.3 The net flow from the cushion cavity is zero. 
5.3.5.4 The total pressure of gas in the trunk and cushion are equal to $P_{j}$ and $P_{\mathrm{C}}$ respectively.

The general approach to the problem was to assume a trunk clearance (d) for given values of trunk pressure $\left(p_{j}\right)$ and recovery pressure ratio $\left(p_{c} / p_{j}\right)$. The jet height for each row of the trunk nozzles was determined from the trunk shape programs developed in Chapter 4. Starting on the cushion side of the trunk, the flow from the $\mathrm{m}^{\text {th }}$ row of jets (see Figure 5-4) was determined. The flow out of the cushion at the $(m-1)^{\text {th }}$ row of jets was assumed to equal the flow into the cushion from the $\mathrm{m}^{\text {th }}$ row of jets. Since the jet height at the $(m-1)^{\text {th }}$ row of jets was known, the velocity and static pressure in the cushion exhaust nozzle at the $(m-1)^{\text {th }}$ row could be calculated. The resulting static pressule was used to determine the flow from the $(m-1)^{\text {th }}$ row of trunk orifices. The flow and pressure at subsequent rows of orifices were determined sequentially in a similar manner until the pressure at the cushion nozzle cxhaust (the $e^{\text {th }}$ row of trunk orifices) was found. If the calculated and assumed value of pressure at the cushion nozzle exhaust did not agree, the trunk clearance (d) was adjusted until agreement was achieved.

The equations for preclicting the pressure distribution across the distributed jets for the restrictor theory are summarized in the following paragraphs.

The jet velocity from the $\mathrm{m}^{\text {th }}$ row of jets (see Figure 5.4 ) may be calculated from Bernoulli's equation.

$$
\left(v_{t}\right\rangle_{m}=\sqrt{P_{j}-P_{c}} \sqrt{\frac{2 g_{o}}{\rho}}
$$

Equation (5-23) gives the jet velocity for the $\mathrm{m}^{\text {th }}$ row of orifices in terms of the known pressure difference across these orifices. The velocity of the gas in the trunk was assumed to be zero and Assumptions 5.3.5.1 and 5.3.5.2 were applied in the development of Equation (5-23). 
The total flow from the $m^{\text {th }}$ row of orifices may be determined by applying the continuity equation.

$$
\left(O_{t}\right\rangle_{m}=\left\langle v_{t}\right\rangle_{m}\langle t\rangle_{m}\left(C_{x}\right\rangle_{m}(s)
$$

The entire flow from the $\mathrm{m}^{\text {th }}$ jet is assumed to exhaust through the plenum exhaust nozzle formed hetween the trunk and the ground. The velocity of the gas in the pienum exhaust nozzle at a section just to the left of the $(m-1)^{\text {th }}$ row of jets (see Figgure 5-4) may be computed from the continuity equation. The resulting relationship is:

$$
(v)_{m-1}=\frac{\left(Q_{t}\right)_{m}}{\left(d+\delta_{m-1}\right)(S)\left(C_{t}\right)}
$$

Equation (5.25) predicts the velocity of the gas in the plenum exhaust nozzle at a section just to the leit of the $(m-1)^{\text {th }}$ row of trunk orifices. The values of $S$ and $C_{t}$ are known and constant for a particular trunk design. The value of $\left(Q_{t}\right\rangle_{m}$ was predicted by Equation (5-24). The value of $\delta_{\mathrm{m}-1}$ may be determined from the trunk shape program developed in Section 4.4. Only the value of $d$ on the right hand side of Equation (5-25) is unknown. The correct value of $d$ is the value which will predict atmospheric pressure at plenum nozzle exhaust plane. At this point it is necessary to assume a trial value of $d$.

The pressure at the $(m-1)^{\text {th }}$ row of trunk orifices may be computed from the total pressure and the gas velocity. Based on Assumption 5.3.5.2, the total pressure at any point in the plenum exhaust nozzle is $P_{C}$. The resulting static pressure at the $(m-1)^{\text {th }}$ jet row is

$$
(P)_{m-1}=P_{c}-\frac{\left(v_{m-1}\right)^{2}}{2 g_{0}}
$$


Equation (5-26) predicts the static pressure at the $(m-1)^{\text {th }}$ row of trunk orifices. Since the static pressure at the $(m-1)^{\text {th }}$ row is known, velocity and flow from the $(m-1)^{\text {th }}$ row of jets may be calculated. In a similar manner to the procedure developed by Equations (5-23) through (5-2B), the pressure distribution for all the remaining jets may be calculated in sequence.

The general equations for the pressure distribution calculation are:

$$
\begin{aligned}
& \left(v_{t}\right)_{m-n}=\sqrt{\frac{2 a_{0}}{\rho}\left(P_{j}-P_{m-n}\right)} \\
& \left(O_{t}\right)_{m-n}=\langle v)_{m-n}(t)_{m-n}\left(C_{x}\right)_{m-n}(S) \\
& (Q)_{m-n}=\sum_{n=0}^{m-l}\left(Q_{t}\right)_{m-n} \\
& (v)_{m-n}=\frac{(Q)_{m-n}}{\left(d+\delta_{m-n}\right)(S)_{\left(C_{T}\right)}} \\
& \text { (P) })_{m-n-1}=P_{c}-\frac{(v)_{m-n} 2(p)}{2 g_{0}}
\end{aligned}
$$

The pressurc at each jet may be calculated in sequence until the minimum pressure and maximum exhaust velocity is reached. The maximum velocity in the exhaust nozzle is determined by the expansion of the exhaust flow from the total cushion pressure to atmospheric pressure (Assumptions 5.3.5.1, 5.3.5.2, and 5.3.5.4). The resulting maximum exhaust velocity is:

$$
\text { (v) } \max =\sqrt{\frac{2 g_{O}}{\rho}} \sqrt{P_{c}-P_{a}}\left(C_{D}\right)
$$


In Equation (5-32), the coefficient $C_{D}$ was introduced to compensate for the convergent-divergent shape of the plenum exhaust nozzle.

The pressure ditribution problem may now be solved on an iterative basis by varying the jet height (d) until the maximum predicted plenum exhaust velocity agrees with the velocity predicted by Equation (5-32).

The procedure is basically the same as outlined in Section 5.2.4. Total jet flow and jet height may be predicted from Equations (5-20) and (5-21) respectively, once the pressure distribution for the distributed jet is known.

\subsection{Analytical Results}

The distributed jet theories require the specification of more design parameters than the concentrated jet theories. In particular, the distributed jet theories require the specification of the trunk shape and the nozzle size, location, spacing, and number. The concentrated jet theoric:; are useful in visualizing general trends. The distributed jet theories are useful in predicting actual performance of a particular distributed jet design.

Because of the large number of variables involved, the analytical results will be presented for one single design. The design selected was the side trunk discussed in Section 4.6 and shown in Figure 4-8. The trunk material is assumed to be inelastic. The nozzles are formed by 8 rows of $5 / 16^{\prime \prime}$ diameter orifices. The spacing between the rows is $1-1 / 4^{\prime \prime}$. The spacing between orifices in a given row is $2-1 / 2^{\prime \prime}$. The location of the rows of orifices on the trunk is determined by specifying $\lambda_{n}$ as shown in Figuse 5-2. The values for $\lambda_{n}$ and the other specified variables are shown in Table 5-I.

The jet height predicted by the distributed jet theories may be compared with the concentrated jet predictions if an equivalent jet thickness is assumed for the distributed jet. The equivalent jet thickness $(\mathbf{t})$ is defined as follows:

$$
t=\sum_{n=1}^{m} \frac{a_{n}}{s}
$$


where:

$a_{n}$ is the total area per row of jets. $S$ is the length of the jet row (trunk section length).

Using the above definition of $t$, the ratio $d / t$ for the distributed jet case becomes equivalent to $d / t$ for the concentrated jet case. It may be noted that:

$$
1 / x=\frac{d}{t(1+\sin \theta)}
$$

Consequently, $1 / x$ and $d / t$ are cqual when $0=0^{\circ}$.

Figure 5-6 gives a comparison of the predicted $d / t$ versus $p_{c} / p_{j}$ for the distributed and concentrated jet theories. For the concentrated jet theories, it was assumed that $0=0$. It is evident from. the figure that the jet height predicted by the flow restrictor theory is considerably lower than that predicted by the various momentum theories.

The relationship between $\mathrm{C}_{\mathrm{Q}}$ and $\mathrm{P}_{\mathrm{c}} / \mathrm{P}_{\mathrm{j}}$ is shown in Figure 5-7. The definition of $\mathrm{C}_{\mathrm{Q}}$ was given by Equation (3-3).

$$
c_{Q}=\frac{Q_{j}}{t s \sqrt{\frac{2 g_{o}}{\rho} p_{j}}} c_{x}
$$

The parameter $\mathrm{C}_{\mathrm{Q}}$ is a flow coefficient which compensates for the pressure variation across the jet. The physical significance of this parameter was discussed in detail in Section 3.4 .

In computing $C_{Q}$, all other flow coefficients were assumed to be unity. The results shown in Figure 5-7 indicate that all distributed jet momentum theories give nearly the same value of $\mathrm{C}_{\mathrm{O}}$. The corresponding values of $\mathrm{C}_{\mathrm{Q}}$ are slightly higher for the distributed jet theories than for the Barratt theory for a concentrated peripheral jet. 


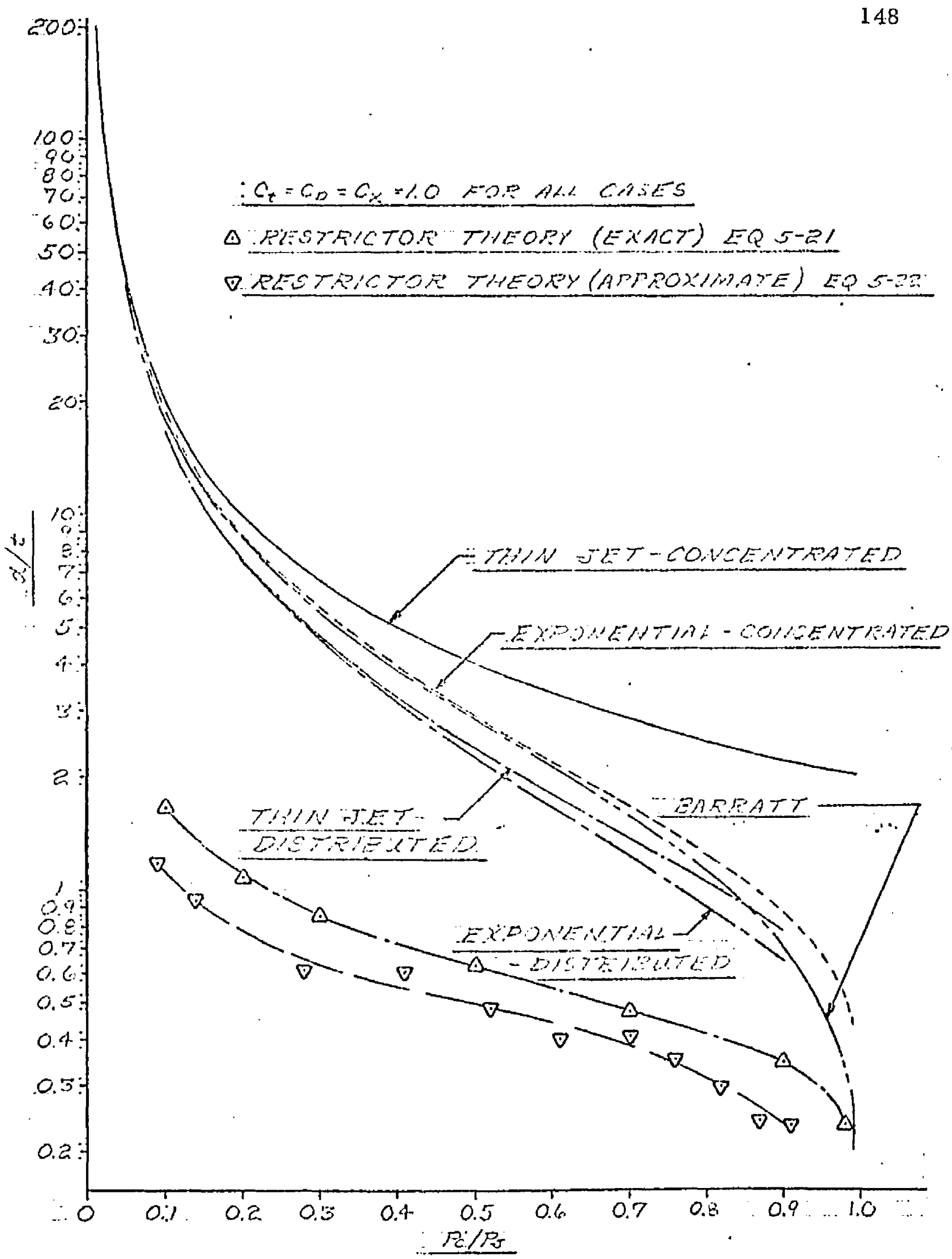

ANALYTICAl! PREDICTIONS OF $d / t$ Vs $P_{c} / P_{j}$-MODEL SIDE TRUNK FIGURE 5-6 


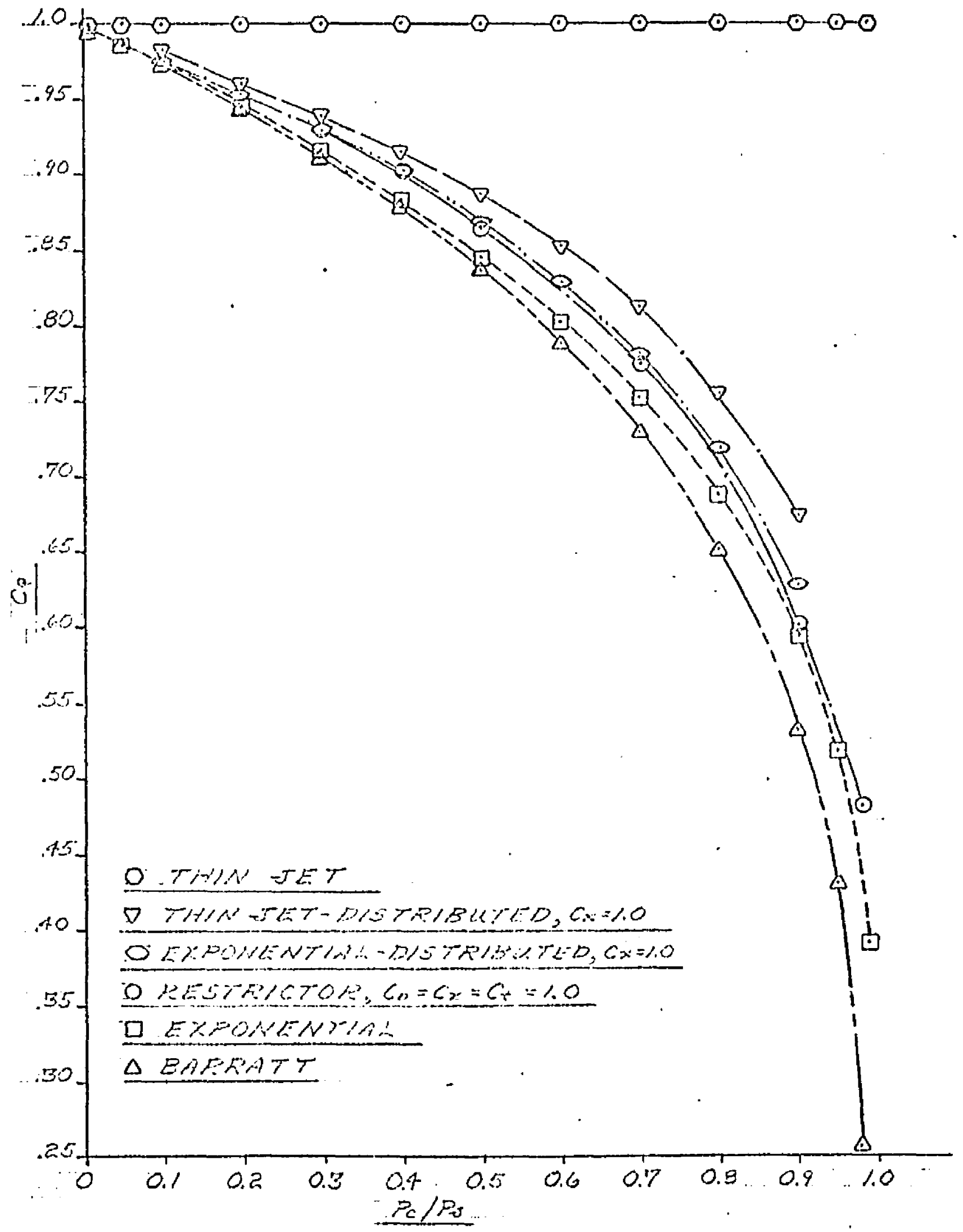

ANALYTICAL PREDICTIONS OF $C_{a}$ vs $p_{c} / P_{J}$-MODEL SIDE TRUNK FIGURE 5-7 
The distributed jet curves presented in Figures $5-6$ and 5.7 were based on an assumed trunk pressure of $120 \mathrm{psig}$. Computations were also made for trunk pressures of 80 psfg and $160 \mathrm{psfg}$. The resulting values of $\mathrm{C}_{\mathrm{Q}}$ and $\mathrm{d} / \mathrm{t}$ were within a few percent of those predicted at $120 \mathrm{psfg}$. It was concluded that the curves presented in Figures 5.6 and 5-7 are dependent only on $p_{c} / p_{j}$ and independent of the magnitude of $p_{j}$. 


\section{EXPERIMENTAL PROGRAM - STATIC MODEL}

\subsection{Experimental Apparatus - Static Tests}

Figure 6.1 sliows the test apparatus used for verification of the trunk shape, flow, pressure distribution, and jet height which were predicted by the analysis developed in Chapters 4 and 5. The plexiglas side in the test rig allowed the inspection of the two-dimensional shape of the trunk cross section. For this reason, the apparatus was generally referred to as the $2 \mathrm{D}$ test rig. The total test apparatus consisted of three units: an air supply, a test section, and a trunk specimen.

Airflow was supplied by a Spenser Gas Booster capable of delivering $3,000 \mathrm{cfm}$ at 1.65 psig. Air was ducted to the test section through 16 feet of 12 -inch diameter galvanized ducting. Trunk pressure was controlled by adjusting a butterfly valve located in the blower housing ahead of the ducting. A flow straightener was positioned in the ducting in accordance with standards set forth in Reference (47). Flow was determined by measuring the differential head across an orifice plate mecting ASME specifications ${ }^{(43)}$ using a micromanometer with a 20 -inch range. Air temperature upstream was measured by a $0-120^{\circ}$ F mercury thermometer.

The test section consisted of a box approximately 32 " wide by $42^{\prime \prime}$ long by 52 " high. The box was constructed from plywood and plexiglis. The front of the box was open to allow air to exhaust and the floor was movable to enable the model to simulate varying vehicle heights. Sixteen static pressure taps, spaced two inches apart, were installed along the centerline of the test section floor.

The trunk specimen under test was made of a nylon-hypalon material which was fastened in the test section by wooden stringers. Six static pressure taps spaced 2-1/2 inches apart were installed along the centerline of the trunk in the jet region. The trunk section was 


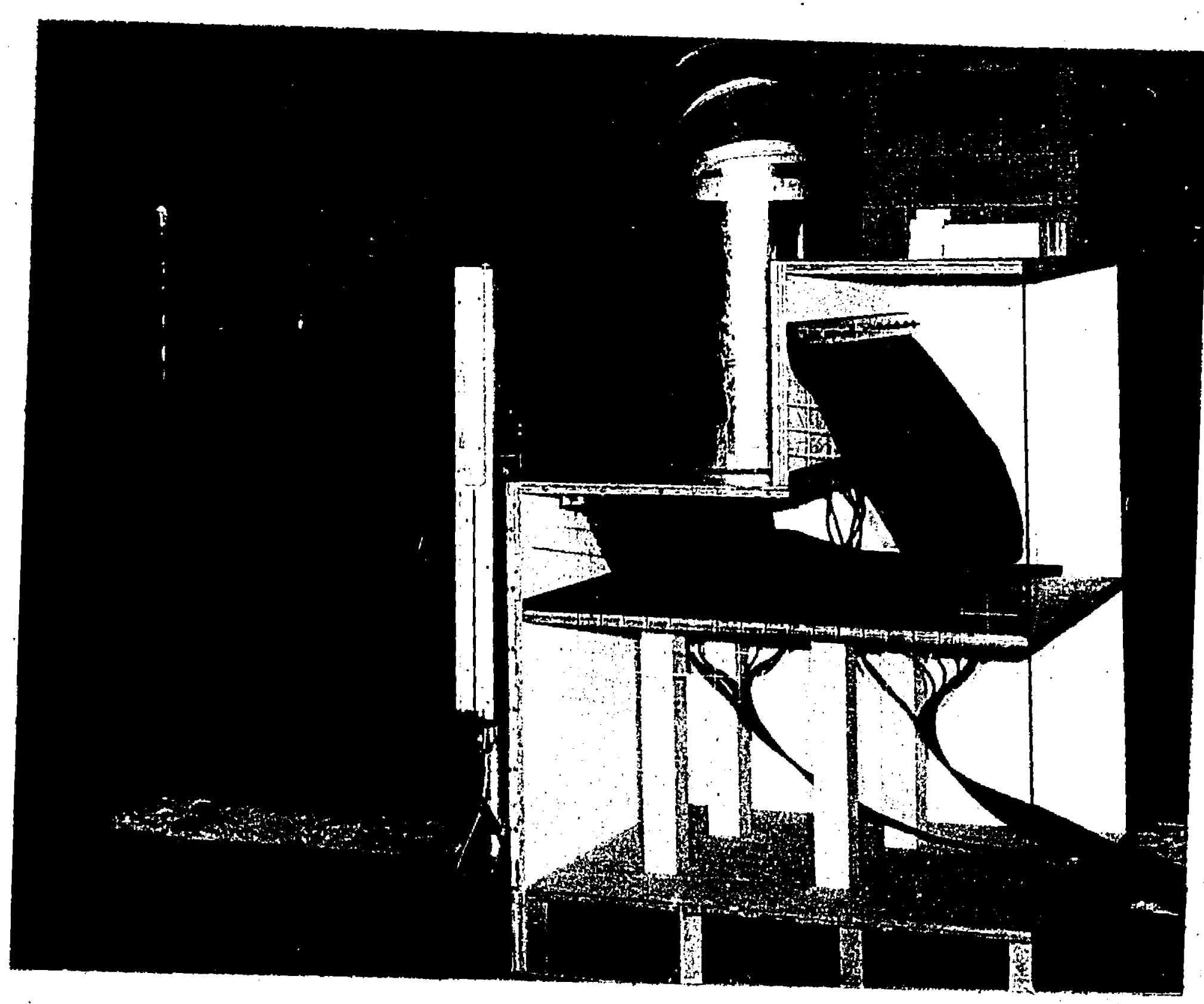

\section{STATIC (2D) TEST RIG FIGURE $6-1$}


32" wide and 57" long. A flap was installed on the edge of the trunk to seal leakage between the trunk and the test section edges. Details of the jet configuration and the trunk elastic properties are given in Appendix IV. The trunk dimensions were the same as those listed in Table 5.1. Consequently, the trunk test specimen represented the side trunk whose shape was analyzed in Chapter 4 and whose flow, pressure distribution, and jet height was analyzer in Chapter 5 .

Airflow was ducted into the trunk through the top of the test section. The air flowed through the trunk, out of the jets, and exhausted through the front of the test section. The flow caused a static pressure to build up between the trunk and the rear of the box when the floor was in place. This pressure was equivalent to the cushion pressure $\left(\mathrm{p}_{\mathrm{c}}\right)$. Both cushion pressure $\left\langle p_{c}\right)$ and trunk pressure $\left(p_{j}\right)$ were measured by pressure taps installed in the top and rear of the test section. All pressure taps wore connected to a 100 -tube well type manometer bank. Water was used as the manometer fluid.

A grid was marked on the plexiglas side of the test section to facilitate observation and measurement of the trunk shape. Trunk shape and low points were measured with a scale.

\subsection{Experimental Procedures - Static Test}

It was necessary to determine the magnitude of leakage flow and the coefficient of discharge for the jets prior to conducting the flow verification tests. The leakage flow was measured by installing in the test suction a trunk specimen without jets and measuring the flow for various values of $p_{j}$ but with $p_{c}=0$. The results of the leakage flow test are summarized in Appendix $V$. The flow coefficient for the jets was measured by repeating the leakage flow procedure after the jets had been installed in the trunk specimen. The results of the coefficient of discharge test are summarized in Appendix VI.

In order to verify the predictions of trunk shape, jet height (d) and pressure coefficient $\left(\mathrm{C}_{\mathrm{Q}}\right)$, tests were conducted on a trunk specimen of the configuration specified in 
Table 5-I. This configuration was identical to the side trunk shape analyzed in Chapter 4 and Chapter 5.

The independent variables in the tests were trunk pressure $\left(\mathrm{p}_{j}\right)$ and vehicle height (H). The vehicle height was set at 10 positions in 1 -inch increments between 4.5 and 13.5 inches. For each vehicle height, the trunk pressure was set at nominal pressures of 40,60 , $80,100,120$, and $140^{\circ} \mathrm{psfg}$. A tolerance of $\pm 2 \mathrm{psf}$ was allowed in the pressure setting. At the beginning of each run, the ambient pressure and temperature were recorderd. The micromanometer which measured the differential pressure across the ASME flow orifice was leveled and zcroed. The vehicle height was set by adjusting the supports for the movable floor. The desired trunk pressure was obtained by adjusting the butterfly valve in the blower housing.

The following data was collected and recorded.

(1) The location of the low point on the trunk was determined by visual sighting and its courdinates were measured from a coordinate system grid with a steel rule.

(2) The jet height was measured by means of calibrated steel rods; the rod was placed on the floor of the model so that its longitudinal axis was parallel to the direction of flow from under the trunk. The rod was then slid under the trunk until it was positioned below the low point of the trunk. Clearance, or the li:k of it, between the rod and the trunk was visually detected and a larger, or smaller, rod was tested for equality of rod diameter and jet height. The rods were calibrated to 0.001 inch in increments of approximately 0.01 inch between 0.03 and 1.00 inch. 

The pressure distributions on the floor and trunk were indicated on the micromanometer bank, as were the cushion and trunk region pressures.

(4) The micromanometer, thermometer, and upstream pressure readings were recorded.

Photographs of the trunk shape were made for a run with $\mathrm{p}_{\mathrm{j}}=80$ and the vehicle height varied in 1.0 inch increments between 13.5 and 4.5 inches.

The results of the tests are summarized in Section 6.3. The variables used in this chapter are summarized in Chapter 5 .

\subsection{Summary of Results - Static Tests}

\subsubsection{Introduction}

Experiments were conducted on a trunk specimen which simulated the side trunk configuration shown in Figure 4.9. This configuration was similar to the side trunk of the model shown in Figure 4-8 whose shape was analyzed in Chapter 4 . The verification of the trunk shape predictions are presented in Section 6.3.2.

The side trunk specimen was also similar to the model analyzed for jet height, pressure distribution and flow in Chapter 5 . The details for this configuration were summarized in Table 5-I. The verifications of the trunk flow characteristics are presented in Section 6.3.3.

\subsubsection{Trunk Shape}

The predicted and experimental values of $\ell_{1}, x_{0}$ and $y_{0}$ for the free trunk shape are shown in Figures 6-2, 6-3, and 6-4 respectively. 


\section{น-9 สมกตม

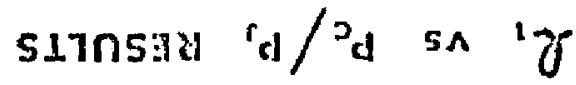

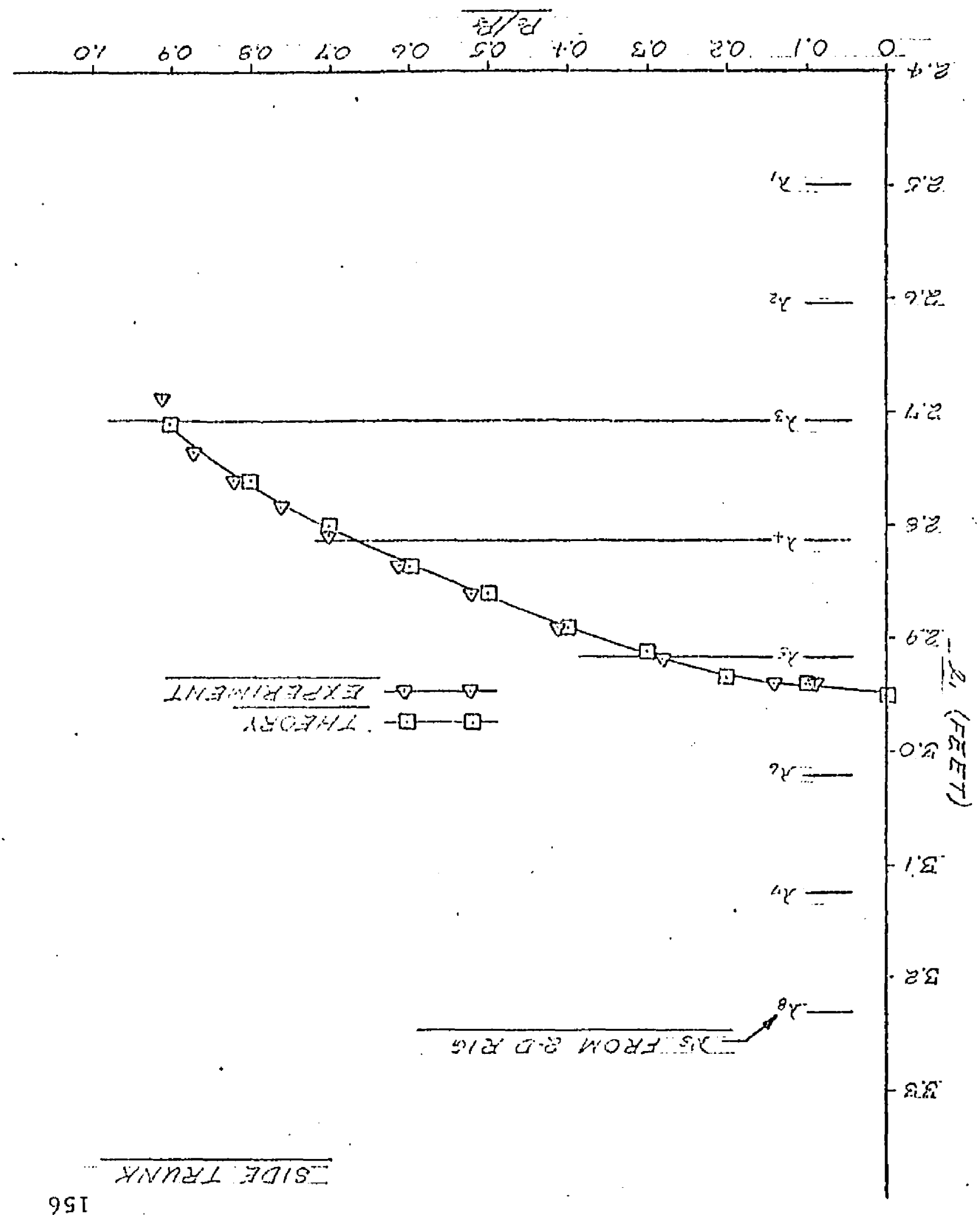




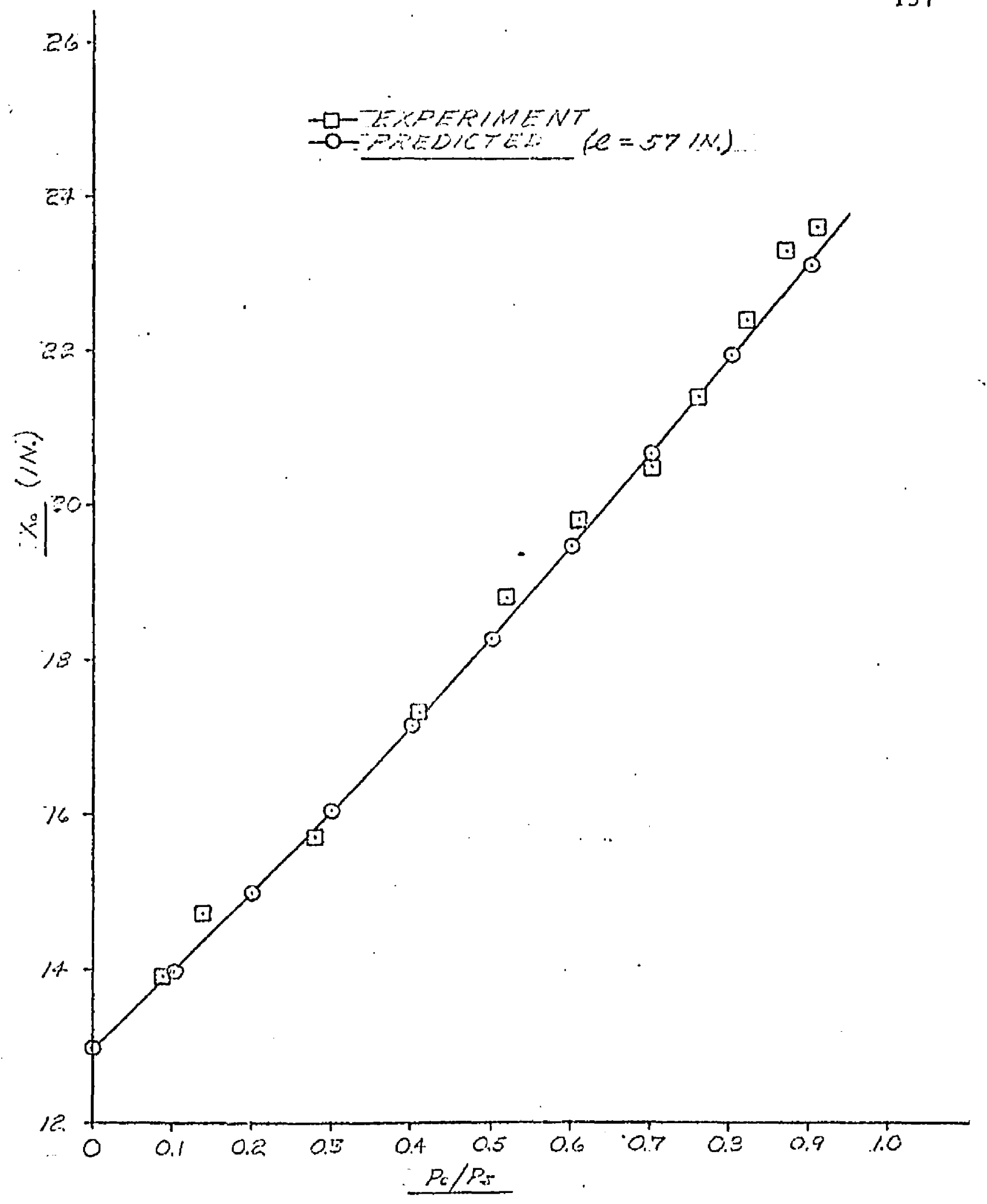

$X_{0}$ vs $P_{c} / P_{J}$ RESULTS

figure o-3 


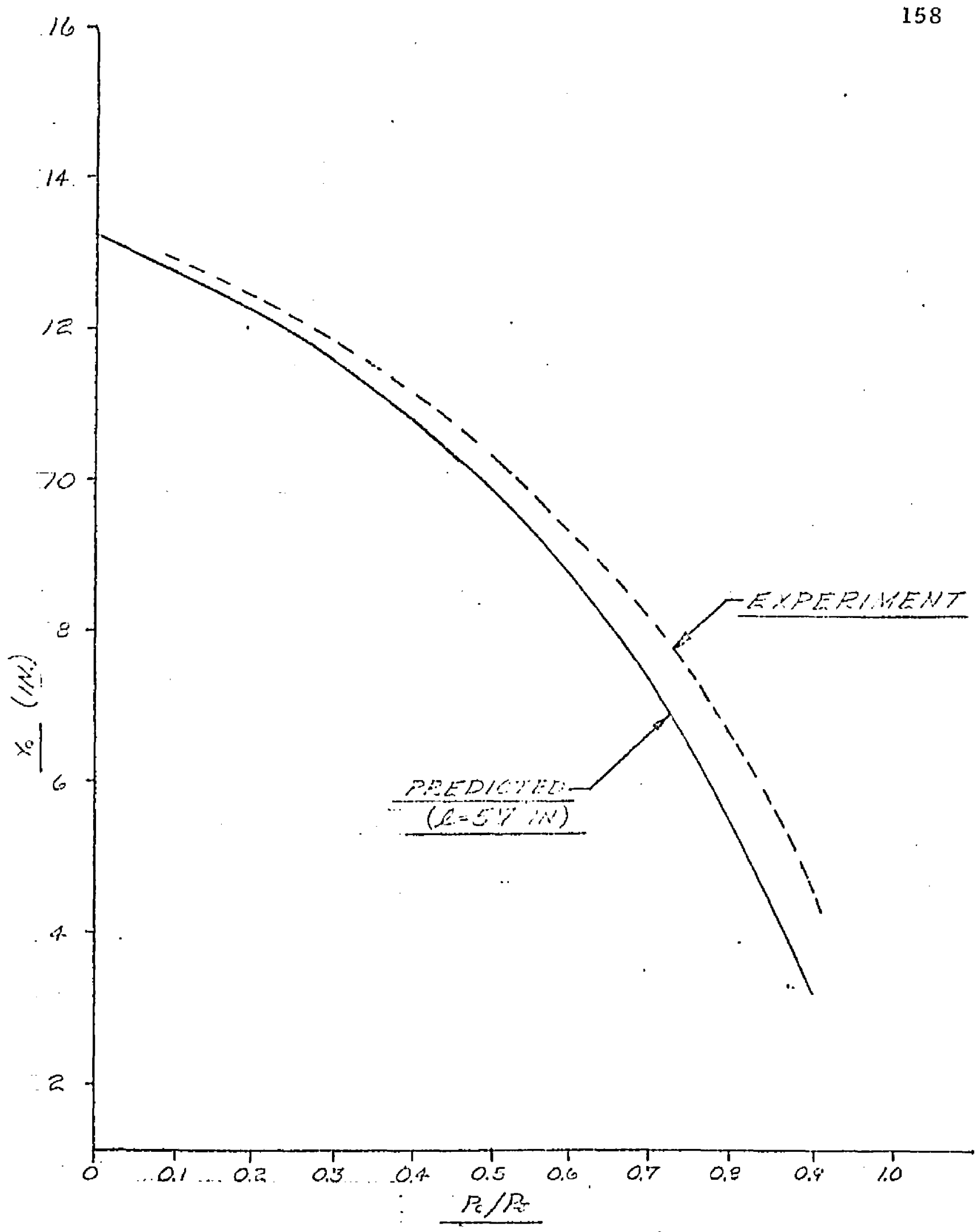

$Y_{0}$ vs $P_{C} / P_{J}$ RESULTS

FIGURE $6-4$ 


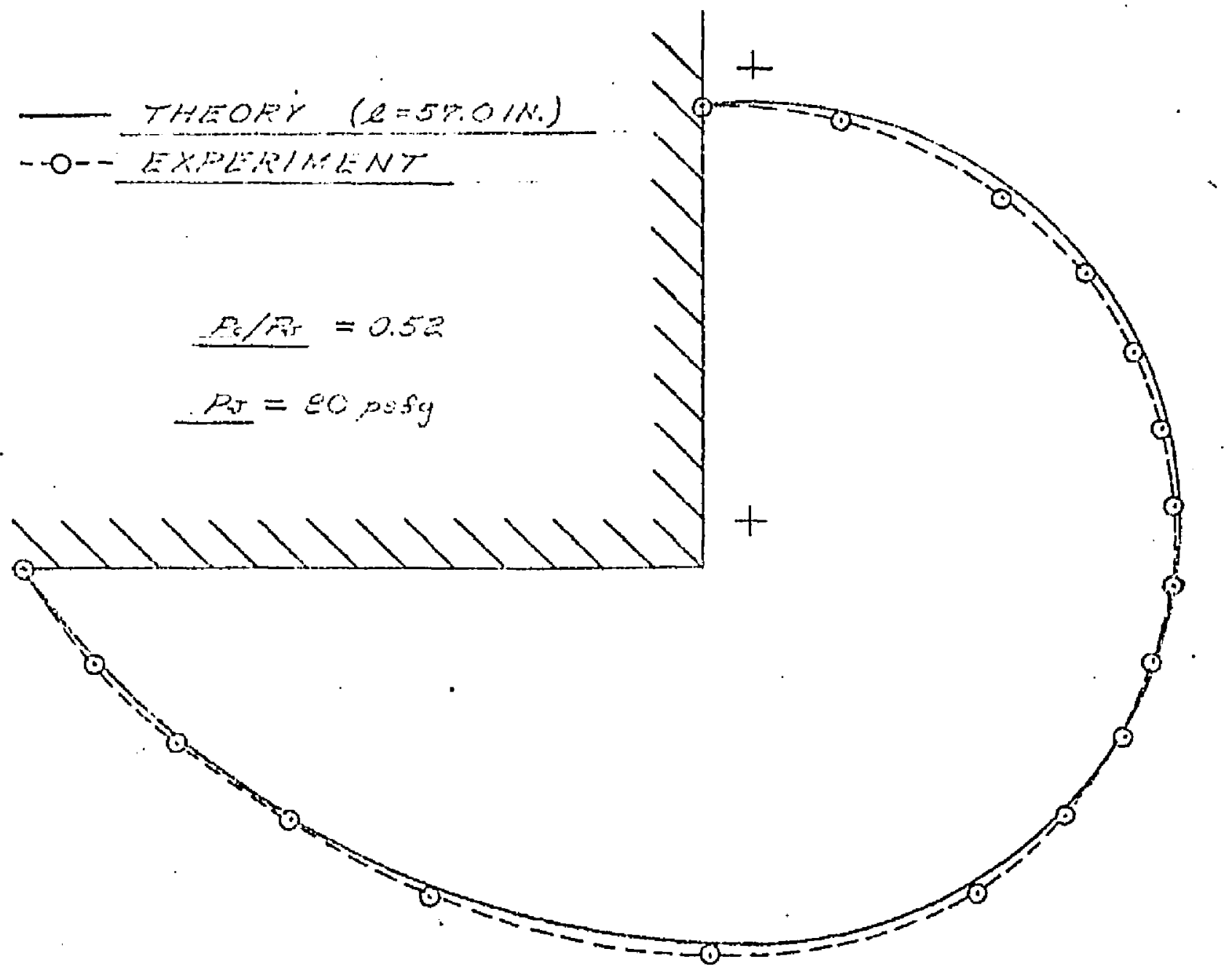


The trunk segment length $\left(\ell_{1}\right)$ is defined as the length of the trunk segment between the attachment point $(a, b)$ and the low point $\left(x_{0}, y_{0}\right)$. This segment is illustrated in Figure 5.4. The length of $l_{1}$ is important in determining the location of the orifices relative to the low point $\left(x_{0}, y_{0}\right)$. The distance from the attachment point $(a, b)$ to the $n^{\text {th }}$ row of orifices is defined by $\lambda_{n}$. For an inelastic trunk, the value of $\lambda_{n}$ is independent of $\mathrm{p}_{\mathrm{c}} / \mathrm{p}_{\mathrm{j}}$ while the value of $\ell_{1}$ is not. The values of the $\lambda_{n}$ 's and $\ell_{1}$ are plotted versu: $\mathrm{p}_{\mathrm{c}} / \mathrm{pj}$ in Figure 6-2. A value of $\lambda_{n}$ greater than $\ell_{1}$ indicates that the $n^{\text {th }}$ row of orifices is on the cushion side of the low point $\left(x_{0}, y_{0}\right)$. Figure 6-2 shows that the number of rows of orifices on the cushion side of the low point varies from 3 at $\mathrm{p}_{\mathrm{c}} / \mathrm{p}_{\mathrm{j}}=1.0$ to 6 at $\mathrm{p}_{\mathrm{c}} / \mathrm{p}_{\mathrm{j}}=0.9$. Close agreement between theory and experiment is shown by the curve.

Figure 6.3 shows the variation of the horizontal position of the trunk low point $\left(X_{0}\right)$ with $p_{c} / p_{j}$. It is evident from the curve that the agreement between theory and experiment is excellent.

Figure 6-4 shows the variation of the vertical position of the trunk low point $\left(\gamma_{0}\right)$ with $\mathrm{p}_{\mathrm{c}} / \mathrm{p}_{\mathrm{j}}$. It is evident from the curve that the agreement decreases as $\mathrm{p}_{\mathrm{c}} / \mathrm{p}_{\mathrm{j}}$ increases. The slight difference between predicted and measured values of $Y_{0}$ was probably due to a vacuum produced just to the atmospheric side of the trunk low point. This phenomena would tend to force the trunk down. The phenomena is discussed in more detail in Section 6.3.2.

Figure 6-5 shows a comparison of the predicted and measured trunk shape for $\mathrm{p}_{\mathrm{c}} / \mathrm{p}_{\mathrm{j}}=0.5$. It is evident that the agreement between theory and experiment for the free trunk shape is excellerit.

In order to determine the validity of the ground loaded trunk shape prediction, a second series of tests was conducted. In this series of tests, the cushion area was vented to the atmosphere. The trunk clearance $\left(Y_{0}\right)$ was varied and the resulting footprint length $\ell_{3}$ was measured with a scale. The resulting values of $\ell_{3}$ versus $Y_{0} / Y_{\infty}$ are compared with the 


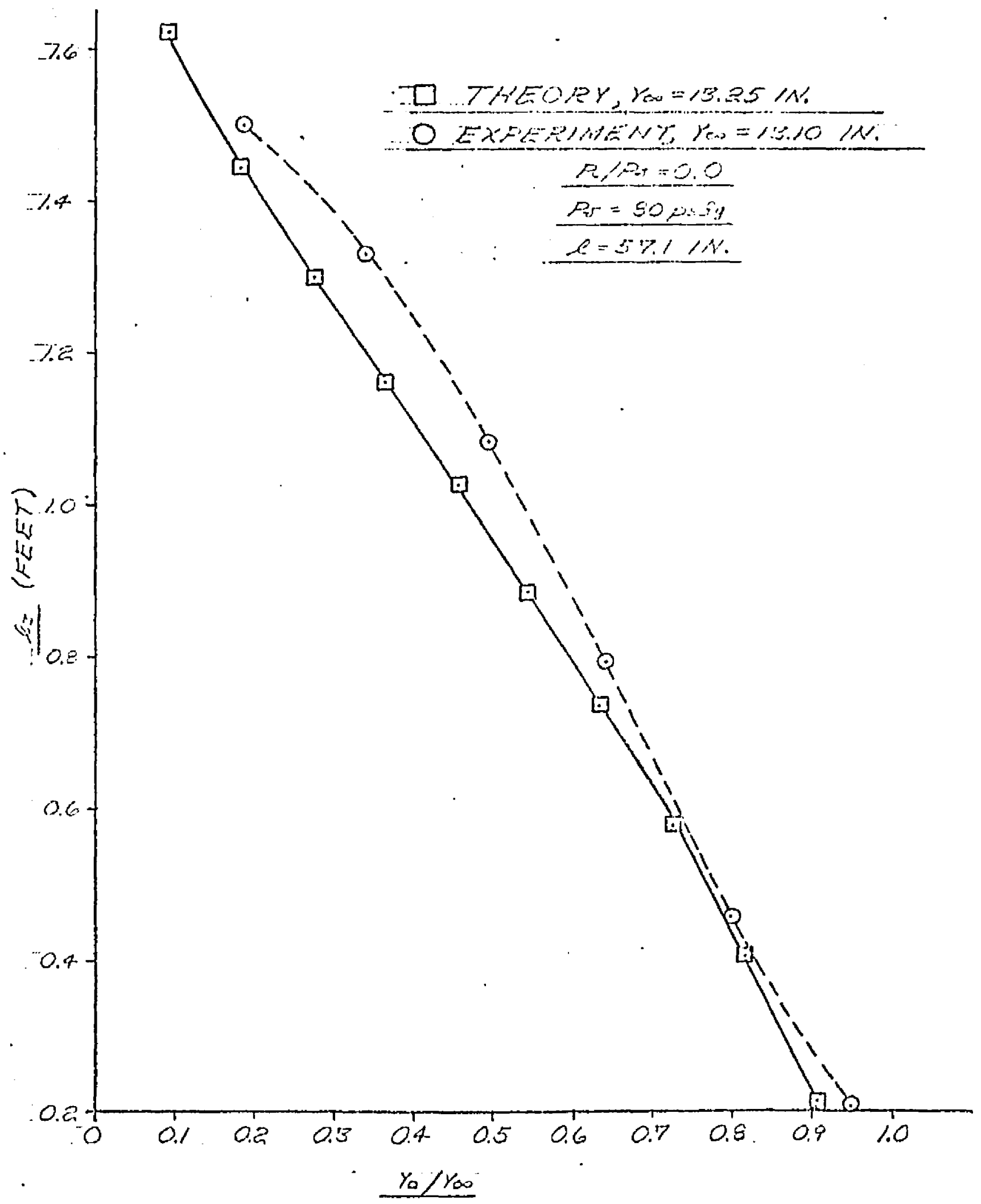

$\ell_{3}$ vs $Y_{0} / Y_{\infty}$ RESULTS, $P_{c} / P_{1}=0$

FIGURE 6-6 


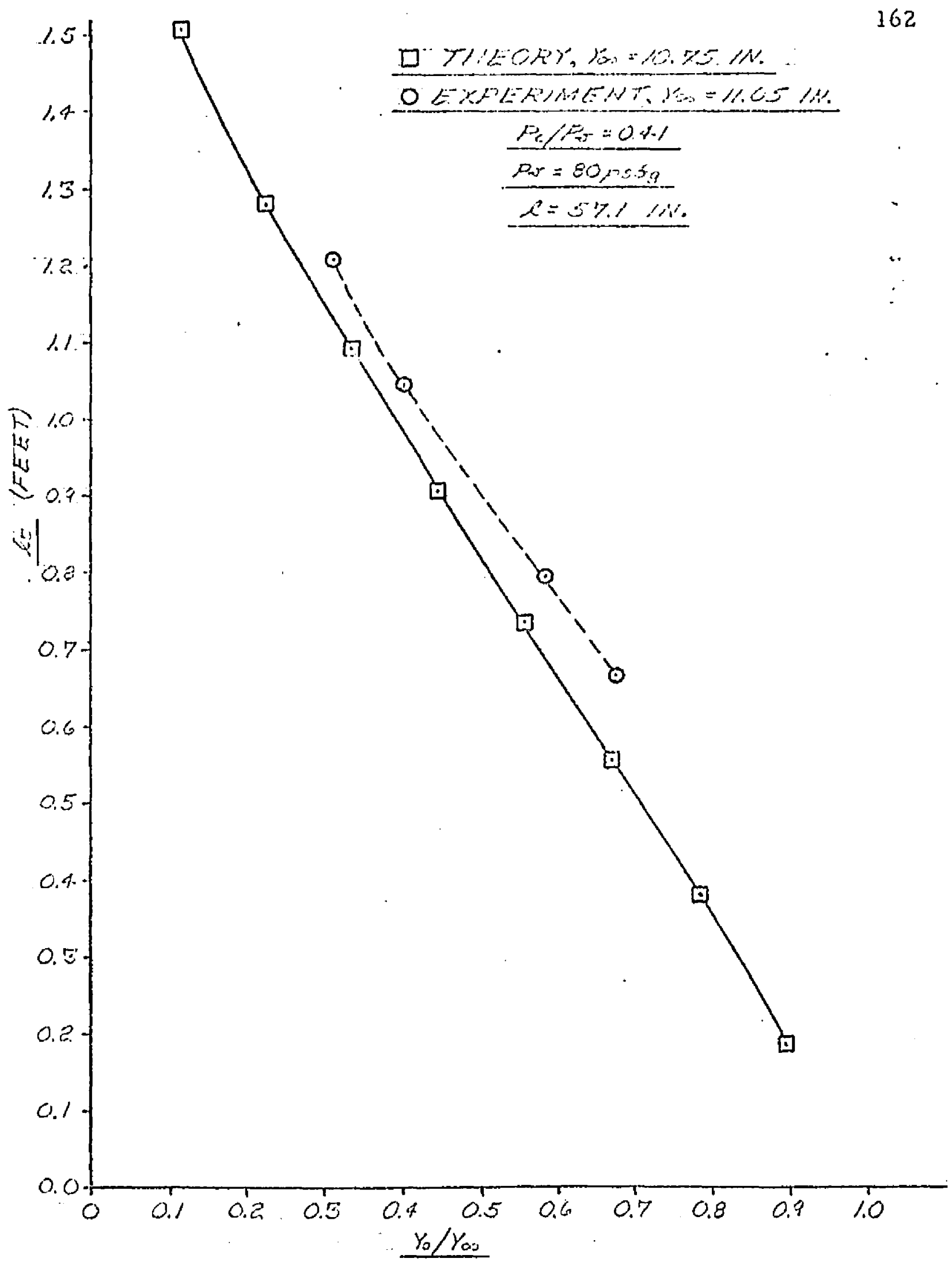

$\ell_{3}$ vs $Y_{0} / Y_{c o s}$ RESULTS, $P_{c} / P_{J}=.41$

FIGURE $6-7$ 
analytically predicted values in Figure 6-6. The figure shows that the agreement between theory and experiment is good for $\mathrm{p}_{\mathrm{c}} / \mathrm{p}_{\mathrm{j}}=0$.

A second run was made with $\mathrm{p}_{\mathrm{c}} / \mathrm{p}_{\mathrm{j}}=0.41$. During this run, the cushion pressure was maintained by introducing flow into the cushion area from a separate air source and venting the resulting cushion flow through the floor of the test section. The resulting values of $\ell_{3}$ versus $Y_{0} / Y_{\infty}$ are compared with the analytically predicted values in Figure 6-7. The figure shows good agreement between theory and experiment.

The trunk shape experiments have demonstrated the accuracy of the analytical models developed in Chapter 4 for predicting the trunk low point, the location of the nozzles, trunk shape, the cross-sectional area, and the footprint length.

\subsubsection{Flow Characteristics}

The results of the tests for leakage flow are shown in Appendix $V$. The experinentally determined flow coefficient for the trunk orifices $\left\{C_{X}\right\rangle$ is given in Appendix VI.

The influence of vehicle (floor) height $(H)$ on $\mathrm{p}_{\mathrm{c}} / \mathrm{p}_{\mathrm{j}}$ is shown in Table 6-1. This table shows that the pressure ratio $\left(\mathrm{p}_{\mathrm{c}} / \mathrm{p}_{\mathrm{j}}\right)$ is largely independent of the trunk pressure $\left(\mathrm{p}_{\mathrm{j}}\right)$.

The influence of vehicle height $(H)$ on the jet height-thickness ratio $(d / t)$ is shown in Table 6-11. The results show that the jet height-thickness ratio $(d / t)$ is not strongly dependent on trunk pressure $\left(p_{j}\right)$.

The influence of vehicle height $(H)$ on the pressure coefficient $\left(\mathrm{C}_{Q}\right)$ is shown in Table 6-111. The results show that $C_{Q}$ is largely independent of trunk pressure $\left(p_{j}\right)$. The method by which $\mathrm{C}_{\mathrm{Q}}$ was calculated is given in Appendix VII. Since the jet height (d) and pressure coefficient $\left(C_{Q}\right)$ are largely independent of $p_{j}$, the presentation of experimental results can be greatly simplified. Table 6-IV shows the average values of the data collected at the various floor heights. These values are assumed to be independent of $p_{j}$. 
TABLE 6-I

Pressure Ratio $\left(\mathrm{p}_{\mathrm{c}} / \mathrm{p}_{\mathrm{j}}\right)$ vs Vehicle Ieight $(\mathrm{H})$ and Trunk Pressure $\left(p_{j}\right)$

\begin{tabular}{|c|c|c|c|c|c|c|c|}
\hline H (in) $\mathrm{P}_{j}(\mathrm{psfg})$ & 40 & 60 & 80 & 100 & 120 & 140 & Ave \\
\hline 4.44 & .91 & .91 & .91 & .91 & .91 & .91 & .91 \\
5.44 & .88 & .88 & .87 & .87 & .87 & .87 & .87 \\
6.44 & .82 & .82 & .82 & .82 & .82 & .82 & .82 \\
7.44 & .76 & .76 & .76 & .76 & .77 & .76 & .76 \\
8.44 & .70 & .70 & .70 & .70 & .70 & .70 & .70 \\
9.44 & .60 & .61 & .60 & .61 & .61 & .61 & .61 \\
10.44 & .52 & .52 & .52 & .53 & .53 & .53 & .52 \\
11.44 & .41 & .41 & .41 & .41 & .41 & .41 & .41 \\
$1 \% .44$ & .28 & .28 & .28 & .28 & .29 & .29 & .28 \\
13.44 & .13 & .14 & .14 & .14 & .15 & .15 & .14 \\
13.94 & .08 & .08 & .09 & .09 & .09 & .09 & .09 \\
\hline
\end{tabular}


TABILE 6-II

Flow Theory Coefficient $\left(C_{\Omega}\right)$ vs

Vehicle Height $(\mathrm{H})$ and Trunk Pressure $\left(\mathrm{p}_{\mathrm{j}}\right)$

\begin{tabular}{|c|c|c|c|c|c|c|c|}
\hline $\mathrm{F}_{(\mathrm{in})} \mathrm{p}_{\mathrm{j}}(\mathrm{ps} f \mathrm{~g})$ & 40 & 60 & 80 & 100 & 120 & 140 & Ave \\
\hline 4.44 & .570 & .581 & .589 & .581 & .580 & .580 &. .58 \\
5.44 & .670 & .665 & .675 & .672 & .667 & .673 &. .67 \\
6.44 & .727 & .730 & .735 & .740 & .736 & .735 &. .735 \\
7.44 & .784 & .791 & .804 & .792 & .797 & .795 & .794 \\
8.44, & .824 & .830 & .830 & .825 & .828 & .825 &. .828 \\
9.44 & .975 & .875 & .870 & .873 & .870 & .870 & .872 \\
10.44 & .920 & .924 & .915 & .920 & .918 & .923 & .920 \\
11.44 & .942 & .950 & .950 & .948 & .953 & .953 &. .950 \\
12.44 & .974 & .990 & .978 & .974 & .974 & .975 & .977 \\
13.44 & .940 & .980 & .980 & .973 & .980 & .975 & .971 \\
13.94 & .975 & .985 & .982 & .983 & .981 & .978 &. .982 \\
\hline
\end{tabular}


TABLE 6-III

Jet Height - Thickness Ratio (d/t) vs

Vehicle I-leight $(\mathrm{H})$ and Trunk Pressure $\left(p_{j}\right)$

\begin{tabular}{|c|c|c|c|c|c|c|c|}
\hline $\mathrm{P}_{\mathrm{j}}$ (ins) & 40 & 50 & 80 & 100 & 120 & 140 & Ave \\
\hline 4.44 & .37 & .37 & .37 & .37 & .39 & .39 & .37 \\
5.44 & .415 & .415 & .435 & .435 & .435 & .435 & .49 \\
6.44 & .46 & .47 & .48 & .50 & .50 & .50 & .48 \\
7.44 & .50 & .52 & .52 & .52 & .545 & .545 & .53 \\
8.44 & $j 58$ & .58 & .58 & .59 & .59 & .60 & .59 \\
9.44 & .62 & .62 & .63 & .64 & .64 & .64 & .63 \\
10.44 & .71 & .71 & .72 & .72 & .72 & .72 & .72 \\
11.44 & .85 & .85 & .85 & .85 & .86 & .87 & .85 \\
12.44 & 1.02 & 1.02 & 1.03 & 1.04 & 1.04 & 1.04 & 1.08 \\
13.44 & 1.59 & 1.57 & 1.57 & 1.57 & 1.57 & 1.59 & 1.58 \\
13.94 & 2.19 & 2.17 & 2.10 & 2.08 & 2.08 & 2.10 & 2.12 \\
\hline
\end{tabular}


TABLE 6-IV

Calculated Data vs Vehicle Height ( $\mathrm{H})$

\begin{tabular}{rllllll}
\hline II (in) & $\mathrm{p}_{\mathrm{c}} / \mathrm{p}_{\mathrm{j}}$ & $\mathrm{C}_{\mathrm{Q}}$ & $\mathrm{d}(\mathrm{in})$ & $\frac{\mathrm{d}}{\mathrm{t}}$ & $\mathrm{x}_{\mathrm{o}}(\mathrm{in})$ & $\mathrm{y}_{\mathrm{o}}(\mathrm{in})$ \\
\hline 4.44 & 0.91 & 0.58 & 0.17 & 0.37 & 23.6 & -4.27 \\
5.44 & 0.87 & 0.67 & 0.19 & 0.42 & 23.3 & -5.24 \\
6.44 & 0.82 & 0.73 & 0.22 & 0.48 & 22.4 & -6.22 \\
7.44 & 0.76 & 0.79 & 0.24 & 0.52 & 21.4 & -7.20 \\
8.44 & 0.70 & 0.83 & 0.27 & 0.59 & 21.0 & -8.17 \\
9.44 & 0.61 & 0.87 & 0.29 & 0.63 & 19.8 & -9.15 \\
10.44 & 0.52 & 0.92 & 0.33 & 0.72 & 18.8 & -10.11 \\
12.44 & 0.28 & 0.98 & 0.48 & 1.04 & 15.7 & -11.97 \\
13.44 & 0.14 & 0.98 & 0.72 & 1.58 & 14.7 & -12.72 \\
13.94 & 0.09 & 0.98 & 0.98 & 2.12 & 13.9 & -12.97
\end{tabular}

NOTE:

The above data exhibited slight variations with $p_{j}$.

The values shown are mean values over the range of $p_{j}{ }^{\prime}$. 


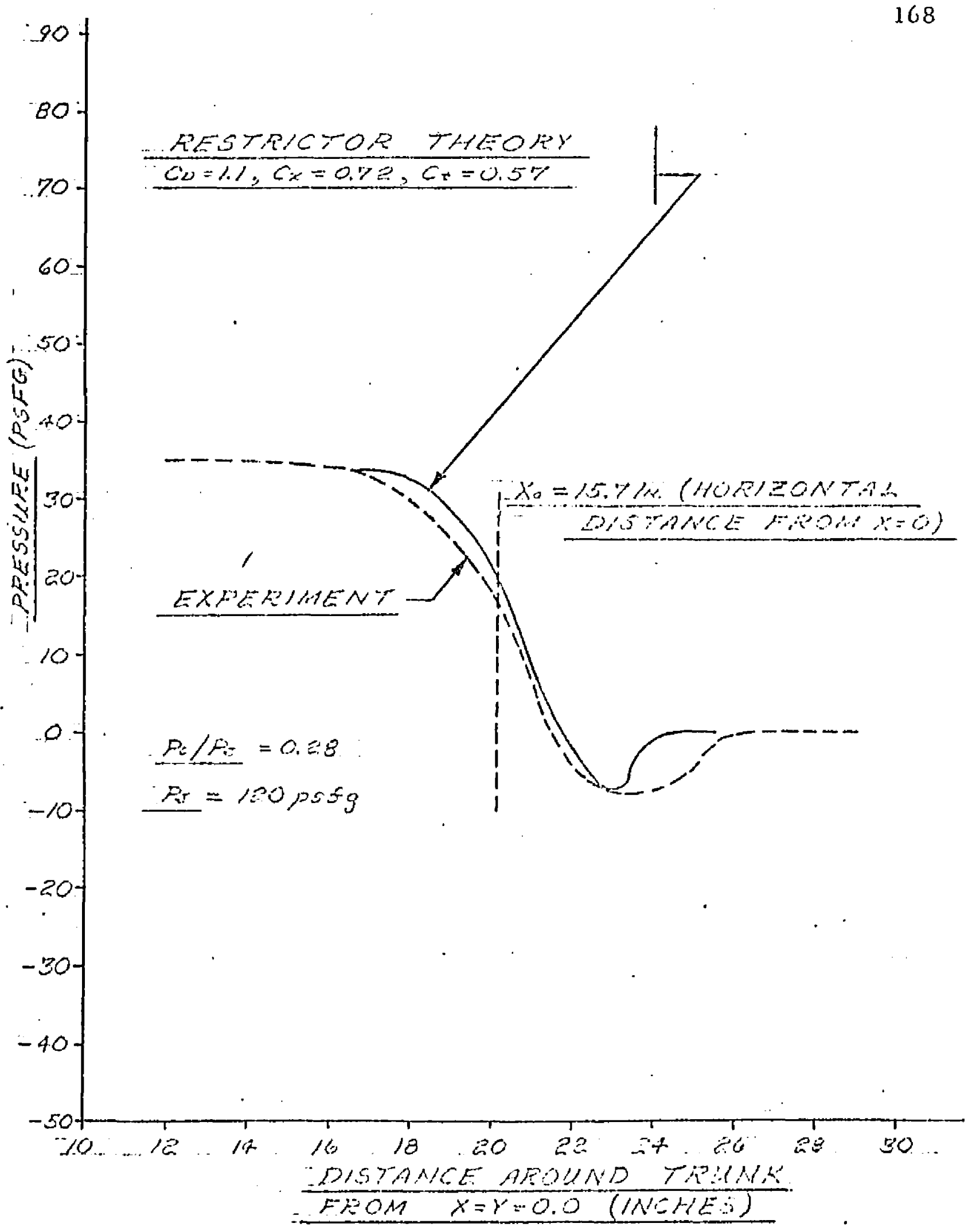

CUSHION EXHAUST PRESSURE DISTRIBUTION, $P_{\mathrm{C}} / \mathrm{P}_{\mathrm{J}}=.28$ FIGURE $6-8$ 


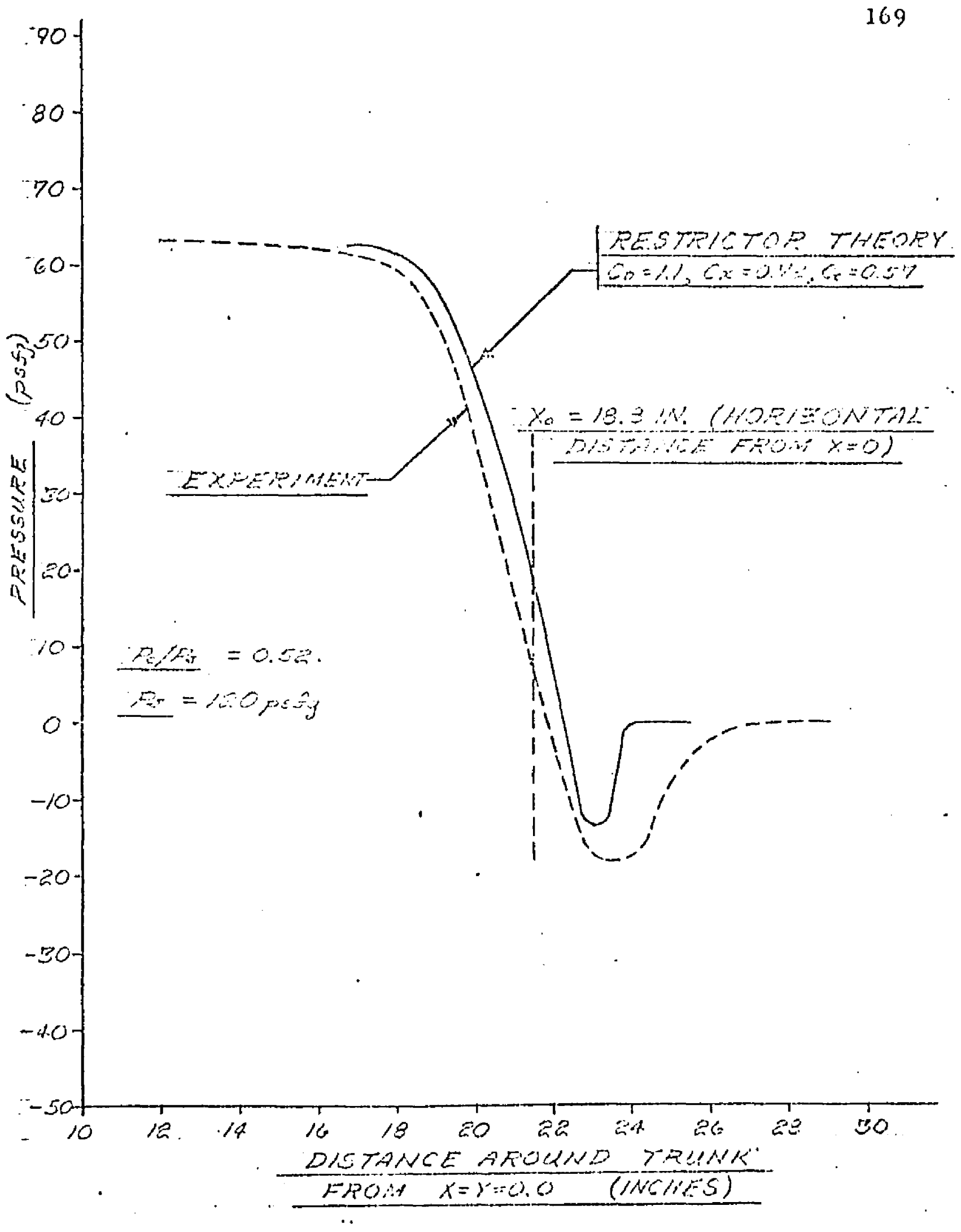

CUSHION EXHAUST PRESSURE DISTRIBUTION, $P_{C} / P_{J}=.52$ FIGURE $0-9$. 


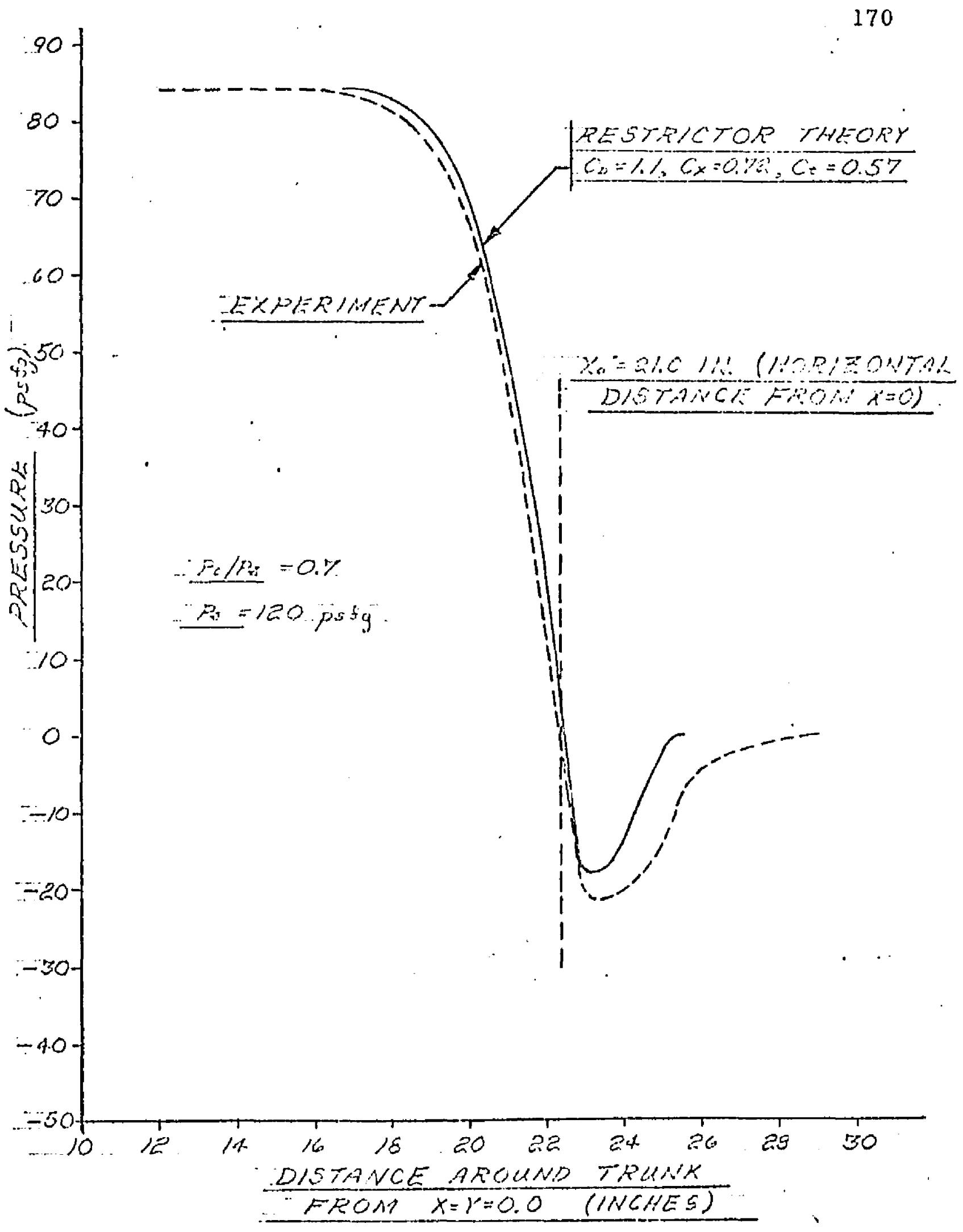

CUSHION EXHAUST PRESSURE DISTRIBUTION, $P_{C} / P_{J}=.7$ FIGURE $6-10$ 
Experimentally measured static pressure distributions along the cushion exhaust nozzle at the trunk surface for the 120 psfg trunk pressure run are shown in Figures 6.8, 6-9, and $0-10$. These figures show the pressure clistributions for pressure ratios $\left(p_{c} / p_{j}\right)$ of $0.28,0.5$, and 0.72 respectively.

Values of $d / t$ and $C_{Q}$ calculated froin experimental measurements in the $p_{j}=120$ psfg run are shown in Figures 6-11 and 6-12 remsectively.

It was found that the value of $\mathrm{C}_{\mathrm{C}}$ predicted by the distributed jet momentum theories was in reasonable agreement with the experimental results. However, the jet height predicted by the momentum theorics was an order of magnitude higher than that observed.

The flow restrictor theory was found to give much better agreement with experimental results. In applying the flow restrictor theory to the experimental model, it was necessary to select values for the three flow coefficients. These coefficients are $C_{X}, C_{D}$. and $\mathrm{C}_{\mathrm{T}}$.

The coefficient $C_{X}$ is the trunk orifice coefficient. The measurement of this coefficient is discussed in Appendix $V I$. The values of $C_{X}$ versus the pressure ratio across the trunk $\left(P_{X} / P_{j}\right)$ are shown in Figure VII-1 (appendix). When cushion pressure is present, the value of $P_{X} / P_{j}$ varies around the trunk. However, since this variation is not large, a constant value of $C_{X}=0.72$ was assumed.

The coefficient $C_{D}$ is intended to evaluate the efficiency of the convergent-divergent nozzle formed between the trunk and the ground in expanding the flow from the plenum chamber to atmospheric pressure. It may be observed from the pressure distribution curves (Figures 6.8, 6-9, and 6-10) that the pressure at the nozzle exhaust is below atmospheric. The flow in this area is highly complex and beyond reasonable analytical analysis. For the shape tested, the vacuum produced in the nozzle exhaust caused the exhaust velocity to be approximately $10 \%$ higher than would have occurred had the minimum pressure been atmospheric. On the basis of these observed results a $C_{D}=1.1$ was selected. 
The nozzle area reduction coefficient $\left(\mathrm{C}_{T}\right)$ represents the effective reduction in plenum nozzle area caused by the momentum seal formed by the jets from the trunk orifices. The high velocity flow from the trunk orifices results in forcing the plenum flow to follow a circuitous path between the jets. The net result is to reduce the effective plenum exhaust nozzle area.

The value of $\mathrm{C}_{\mathrm{T}}$ should be less than 0.76 based upon constant width jets. The width is assumed to be equal to the orifice diameter $\left(5 / 16^{\prime \prime}\right)$ and the minimum distance betwreen jets is $0.965^{\prime \prime}$. The value of 0.76 probably represents an upper bound since the effective area reduction is expected to be greater than the projected width of the orifices. $A$ selection of $\mathrm{C}_{\mathrm{T}}{ }^{\cdot}=0.57$ gave the best agreement with experimental data.

The computed results for the flow restrictor theory using the selected discharge coefficients are shown in Figures 6.8 through 6-10.

It may be seen from Figures 6-8, 6-9, and 6-10 that the agreement between experimentivi and calculated pressure distribution around the trunk is quite good.

The rcsulting jet height to thickness ratio $(\mathrm{d} / \mathrm{t})$ is shown in Figure 6-11. Again, agreement between calculated results and experimental data is excellent. Figure 6-11 also shows the predicted values of $\mathrm{d} / \mathrm{t}$ using the approximate formula (Equation 5-22). The approximate formula gives the correct trend but predicts a lower jet height than is actually observed.

The experimental and calculated values for $C_{Q}$ are shown in Figure 6-12. The flow restrictor theory is she in to give the closest agreement with experiment.

The trunk flow experiments have demonstrated the accuracy of the flow restrictor theory developed in Chapter 5 for predicting the pressure distribution, jet height and flow coefficient of the trunk design under test. The distributed jet momentum theorics were unsatisfactory for predicting the jet height for the tested jet configuration. 


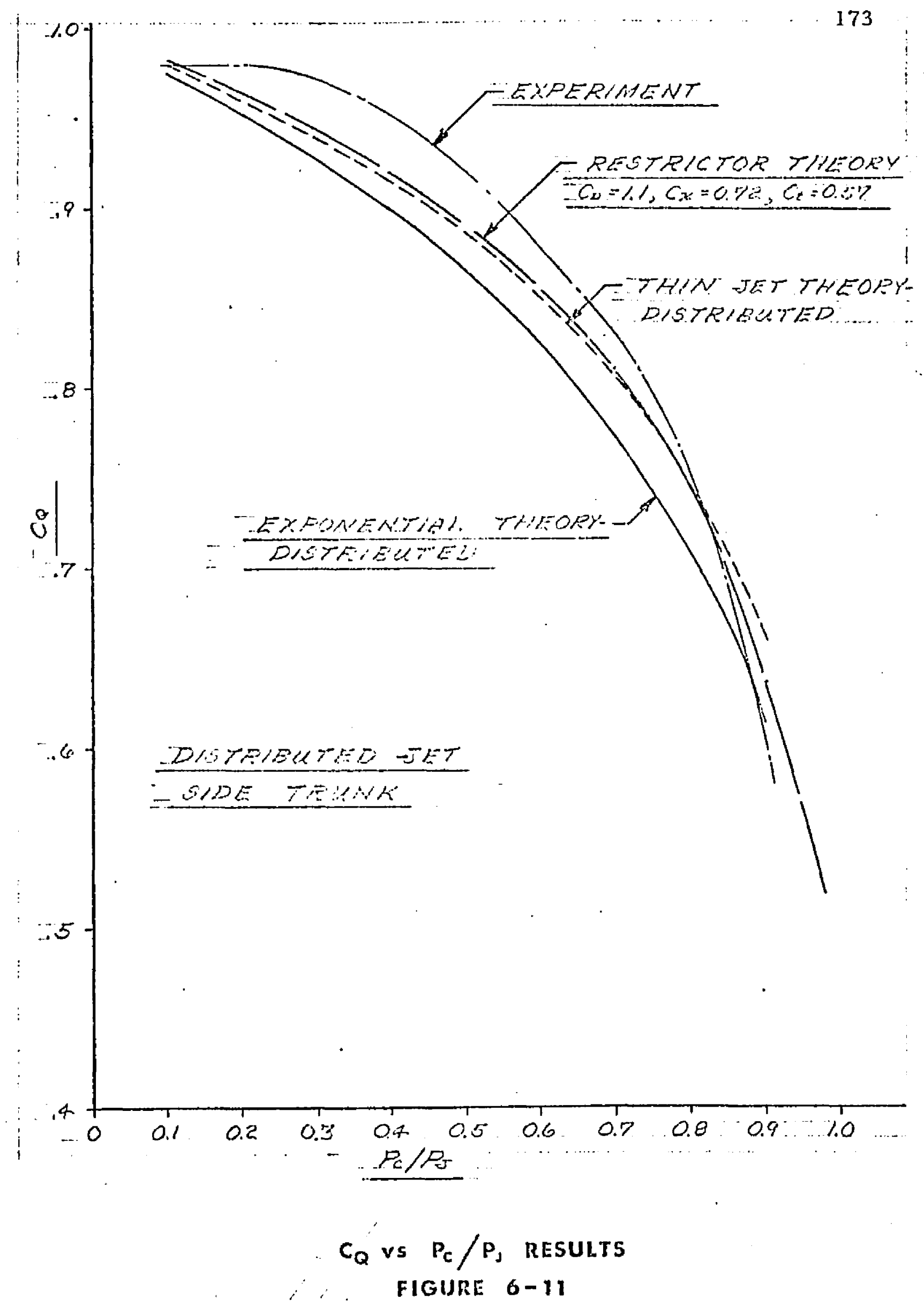


174

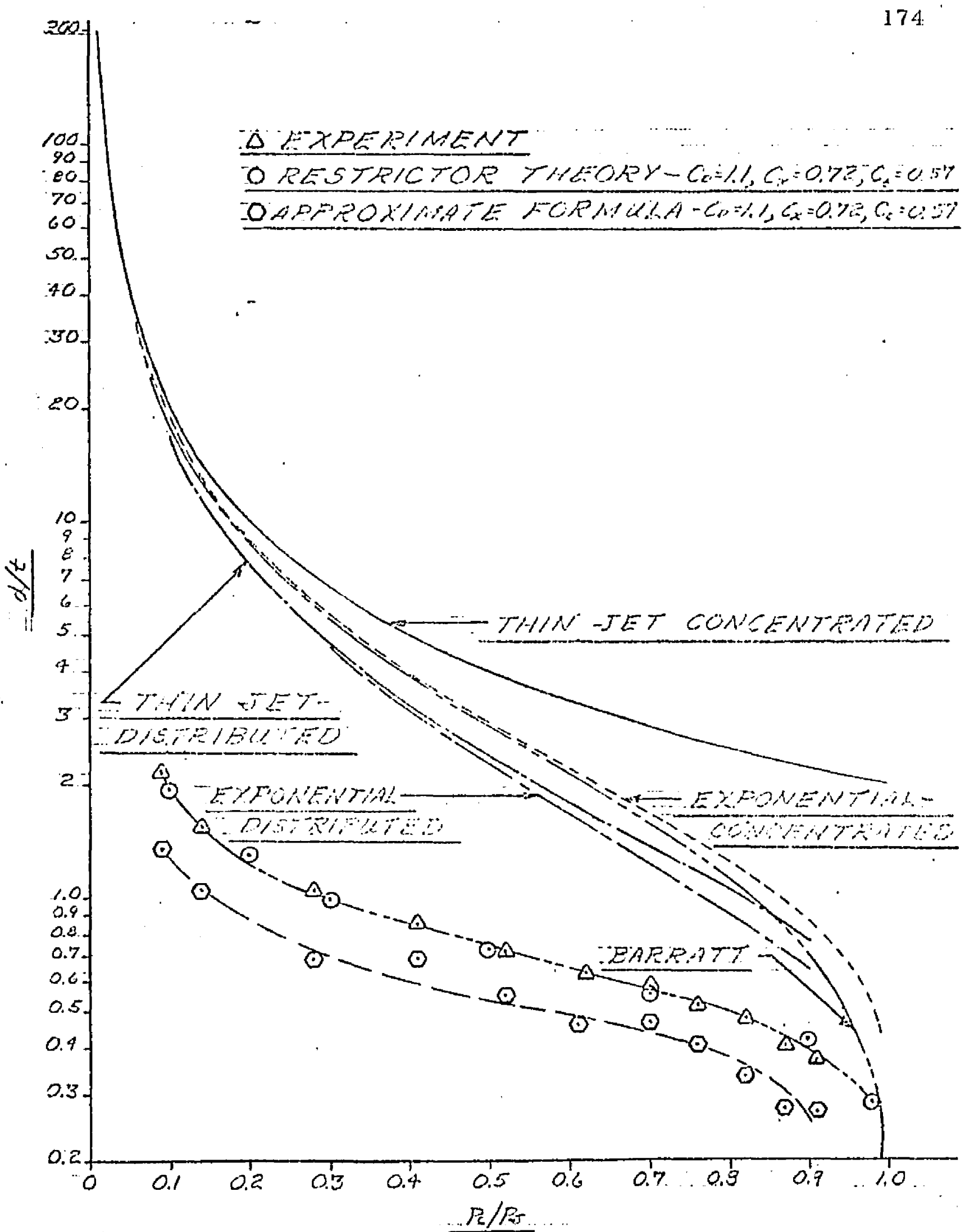

$$
\begin{gathered}
d / t \text { vs } P_{C} / P_{J} \text { RESUETS } \\
\text { FIGURE } 6-12
\end{gathered}
$$




\section{DYNAMIC ANALYSIS OF THE AIR CUSHION LANDING SYSTEM}

$7.1 \quad$ Introduction

The landing gear of an aircraft must have the capacity to absorb the vertical landing energy of the aircraft without overloading the aircraft structure. Two critical parameters in designing the gear are the maximum " $g$ " load and maximum stroke which results from landing at a given weight and sink speed. Consequently, it is clesired to predict the load-stroke characteristics of the Air Cushion Landing System as functions of aircraft weight and vertical velocity.

The aircraft attitude and forward velocity at touchdown also exert an appreciable influence on the load-stroke characteristics of conventional landing year. For the purposes of the analysis presented in this chapter, these two influences are neglected. The pitch and roll angles at touchdown are assumed to be zero, and the forward velocity is assumed to be negligible.

The system of equations which describe the dynamic response of the ACLS is developed in the following sections. Section 7.2 presents a simplified model of the trunk portion of the system. Section 7.3 presents a more complete model of the trunk; but neglects the effect of pressure build-up in the plenum beneath the aircraft. Section 7.4 presents a model of the combined trunk-plenum system.

The variables involved in the analysis are as follows:

A piston area, $\mathrm{ft}^{2}$

$\mathrm{A}_{\mathrm{g}} \quad$ cushion area under the trunk, $\mathrm{ft}^{2}$

$A_{h} \quad$ cushion area under the aircraft hard structure, $\mathrm{ft}^{2}$

$\mathrm{A}_{3}$ truck footprint area, $\mathrm{ft}^{2}$ 


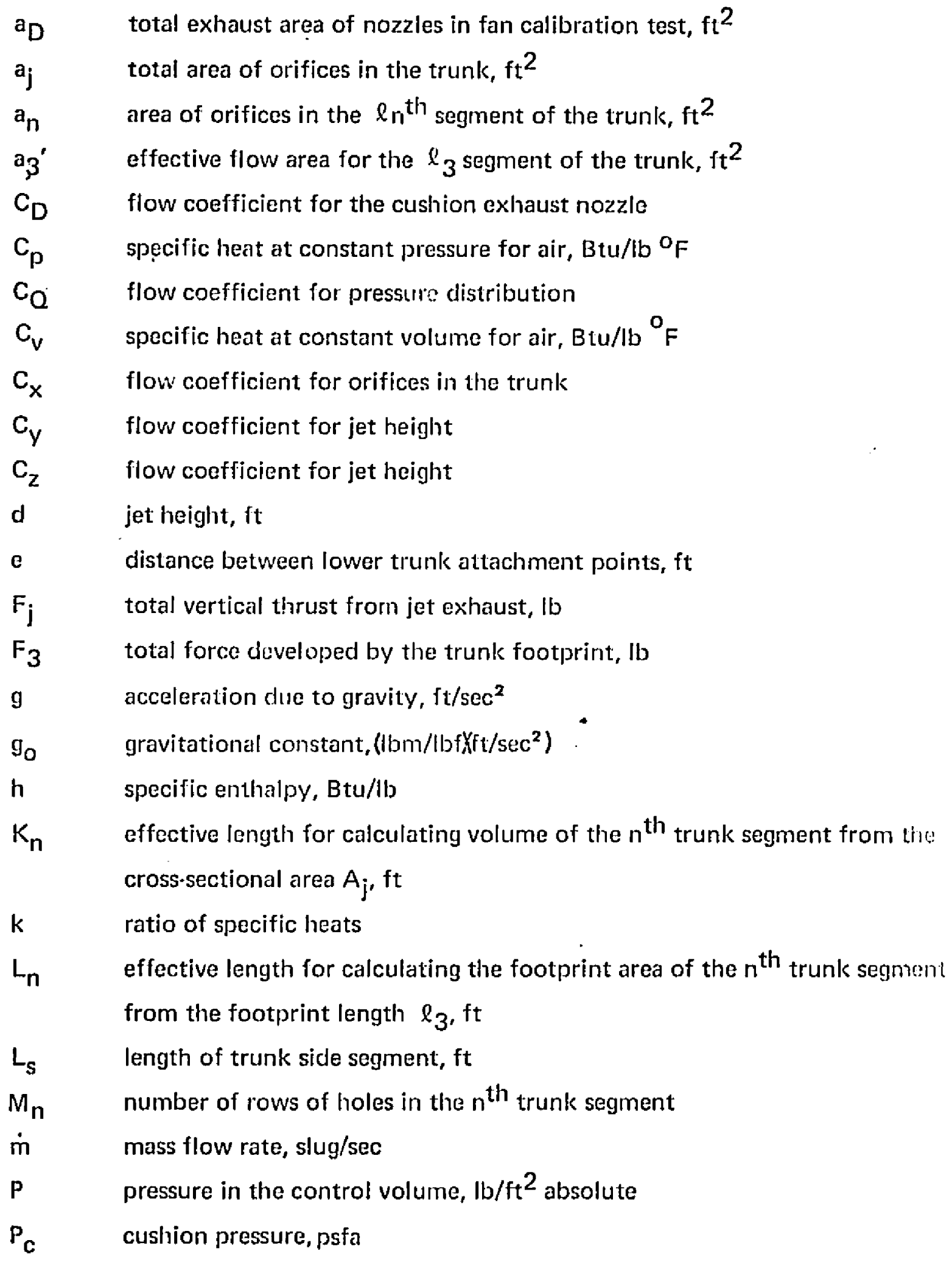




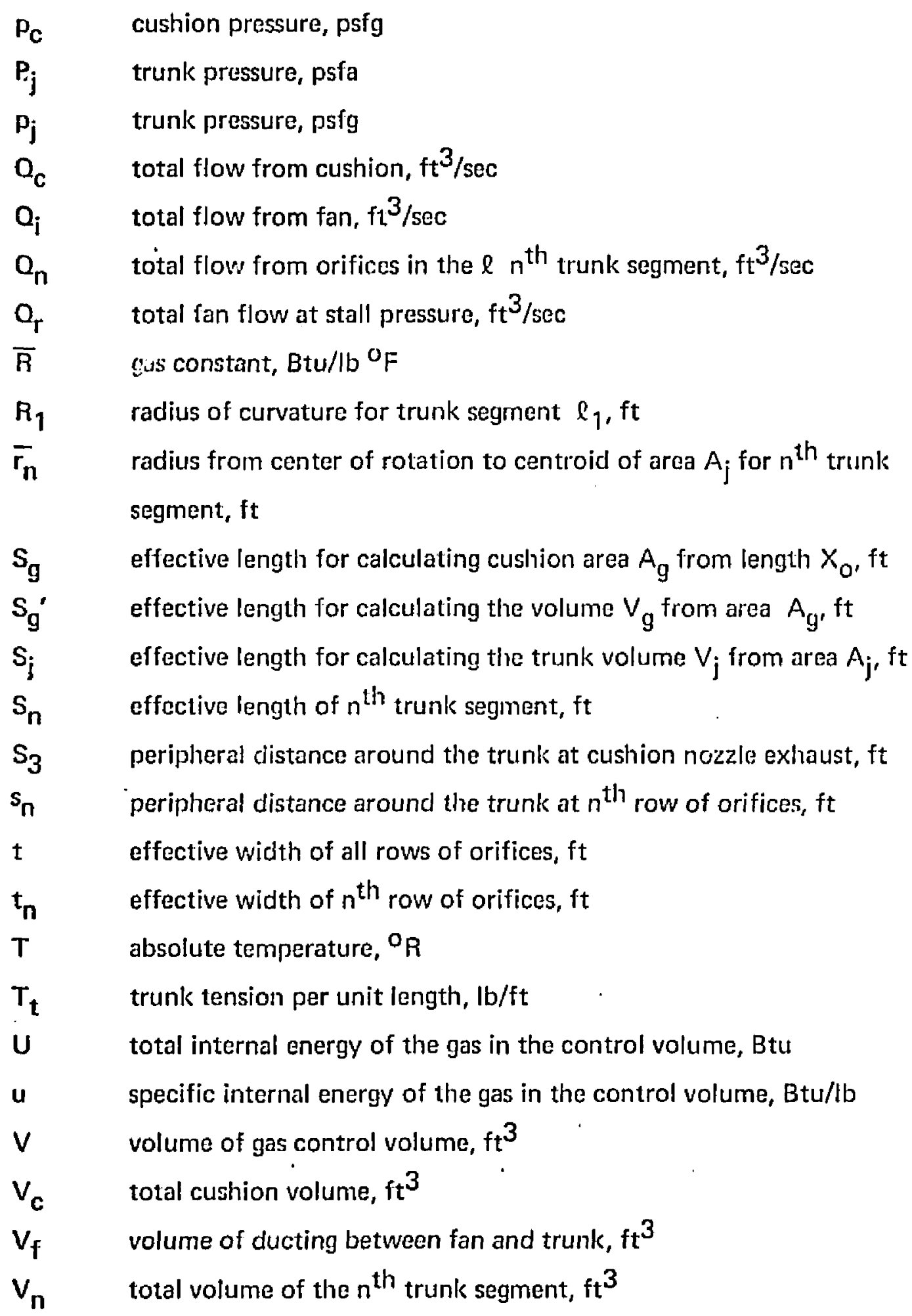




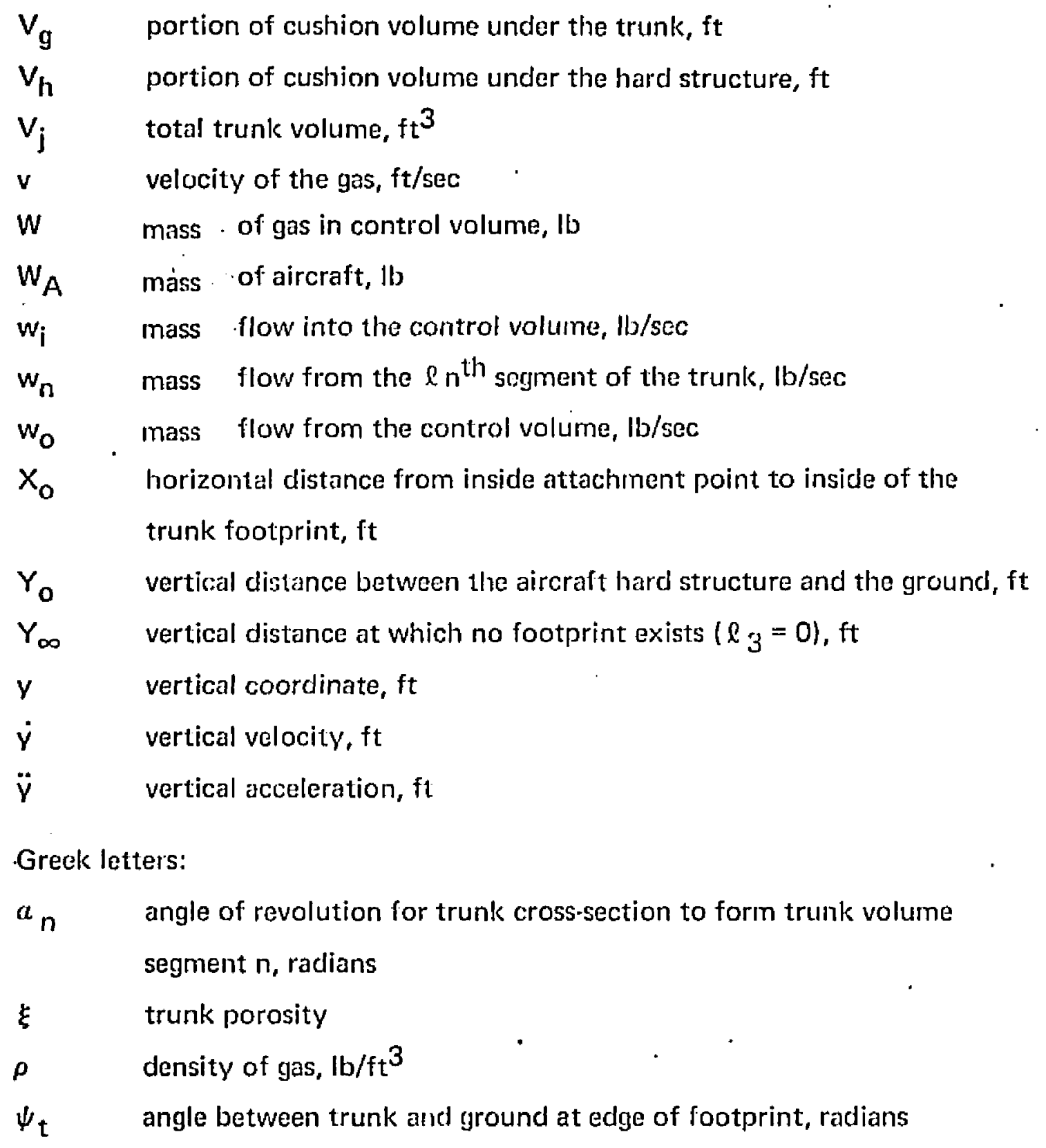

\section{Subscripts:}

c refers to the cushion

e refers to the cnd trunk segment

i refers to flow into the control volume 

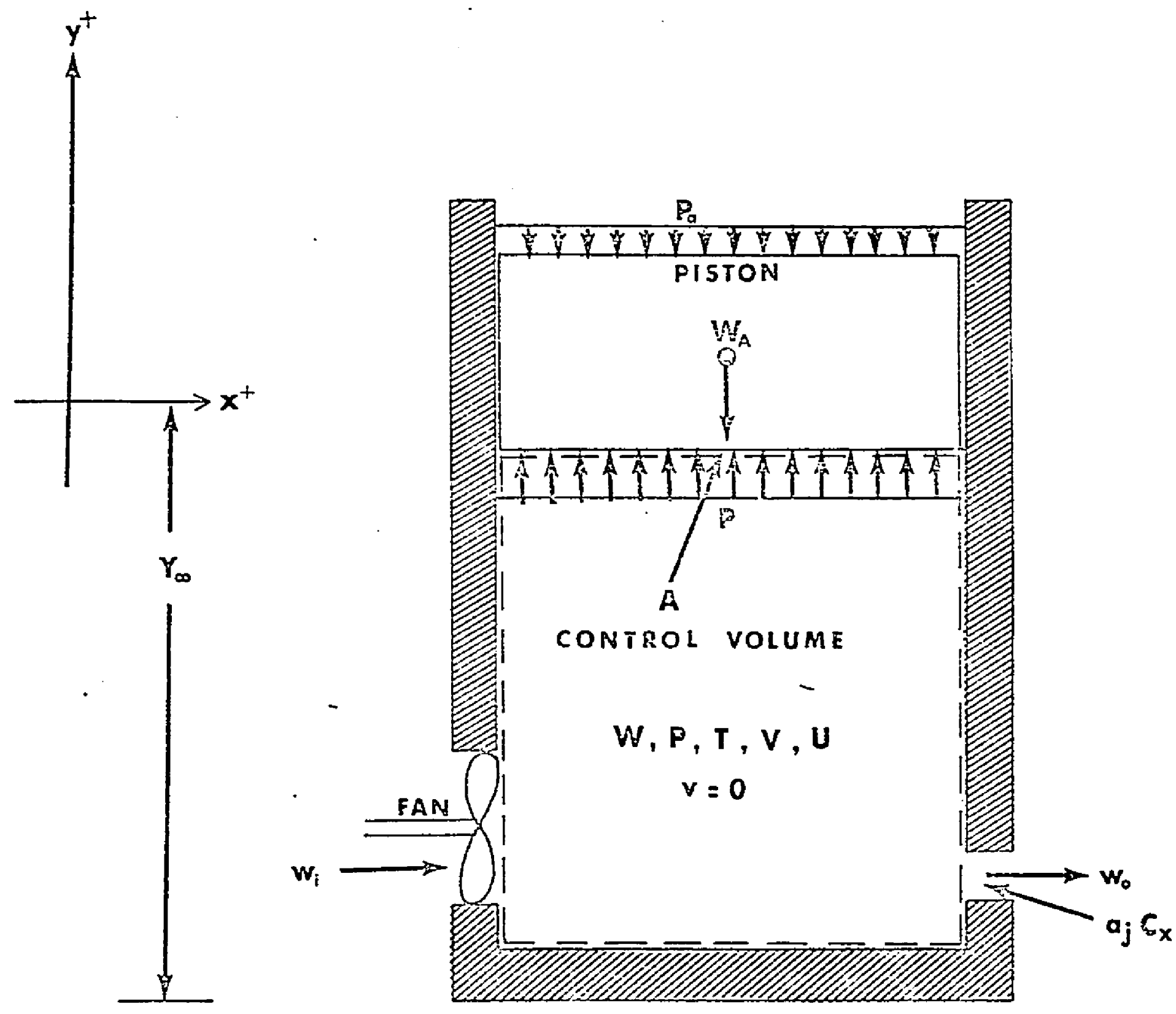

SIMPLE MODEL FOR DYNAMIC ANALYSIS 


$\begin{array}{ll}\text { j } & \text { refers to the trunk } \\ k & \text { refers to the corner trunk segment } \\ n & \text { arbitrary } \\ \text { o } & \text { refers to flow out of the control volume } \\ \text { r } & \text { refers to stall condition of the fan } \\ \text { s } & \text { refers to the side trunk segment } \\ 1 & \text { refers to the segment } \ell_{1} \\ 2 & \text { refers to the segment } \ell_{2} \\ 3 & \text { refers to the segment } \ell_{3}\end{array}$

7.2 Simple Dynamic Model

\subsubsection{Approach and Assumptions}

A greatly simplified model of the air cushion trunk is shown in Figure 7-1. The figure shows an insulated cylinder of gas. The gas is being compressed by a piston falting under the action of gravity. During the compression process, air may enter the control volume from a fan and may leave the volume through an orifice.

The assumptions for the analysis are as follows:

7.2.1.1 Thrust from the exhaust gas is neglected.

7.2.1.2 Adiabatic expansion or compression occurs in the control volume.

7.2.1.3 The change in height of the gas flowing through the control volume is neglected.

7.2.1.4 The enthalpy of the input air equals the enthalpy of the exhaust air. 
7.2.1.5 The gas obeys the perfect gas law.

7.2.1.6 The flow through the exhaust orifice is assumed to be adiabatic and incompressible.

7.2.1.7 The velocity of the gas in the control volume is negligible. The static and total prezsure are equal.

7.2.1.8 The flow in, $a_{i}$ is constant.

The variables involved in this model may be grouped as follows:

Independent environmental variables

g acceleration due to gravity, $\mathrm{ft} / \mathrm{sec}^{2}$

$\mathrm{P}_{\mathrm{a}} \quad$ atmospheric pressure, $\mathrm{lb} / \mathrm{ft}^{2}$

$\rho \quad$ atmospheric density, $\mathrm{lb} / \mathrm{ft}^{3}$

$k \quad$ ratio of specific heats for gas

Independent design variables

A piston area, $\mathrm{ft}^{2}$

$a_{j} \quad$ orifice area, $\mathrm{ft}^{2}$

$C_{X} \quad$ coefficient of discharge of orifice

$Y_{\infty} \quad$ distance of origin of coordinate system above ground, $\mathrm{ft}$

Independent operating variables

$\mathrm{O}_{\mathrm{i}} \quad$ flow from the fan into cylinder, $\mathrm{ft}^{3} / \mathrm{sec}$

$W_{A}$ piston weight, ib 
Time dependent variables *

$\mathrm{P}(\mathrm{t}) \quad$ control volume pressure, $\mathrm{lb} / \mathrm{ft}^{2}$

$T(t) \quad$ control volurne gas temperature, ${ }^{\circ} R$

$V(t) \quad$ control volume, $\mathrm{ft}^{3}$

$W(t) \quad$ control volume gas weight, lib

$\mathrm{v}\langle\mathrm{t}\rangle \quad$ piston position, $\mathrm{ft}$

$\dot{y}(t) \quad$ piston velocity, $\mathrm{ft} / \mathrm{sec}$

In order to determine the variation of the dependent variables with the time parameter, six independent equations are necessary. These equations may be developed by applying the following laws and principles:

(1)

Newton's second law applied to the free piston body in the vertical direction gives:

$\ddot{y}(t)=f[P(t)]$

(2) Geometric compatibility applied to the control volume gives:

$y(t)=f[V(t)]$

(3) An energy balance applied to the control volume gives:

$T(t)=f[W(t), V(t), P(t)]$

14) The perfect gas law gives:

$T(t)=f[W(t), V(t), P(t)]$

(5) The continuity and energy principles applied to flow through the orifice and fan gives: 


$$
W(t)=f[P(t)]
$$

It may be noted that the equation resulting from principles (3) and (4) ray be combined to eliminate $T(t)$. An additional equation defining $\dot{y}(t)=f[y(t)]$ may be introduced to eliminate $\ddot{\gamma}(t)$ from the relationship in principle (1).

\subsubsection{Newton's Second Law}

Newton's second law may be applied to the piston shown in Figure 7-1. The result is:

$$
\frac{W_{A}}{g_{0}} \frac{d^{2} y}{d t^{2}}=-W_{A} \frac{g}{g_{0}}+A\left(P-P_{A}\right)
$$

Equation (7-1) equates the vertical external forces acting on the piston to the product of the mass and acceleration in the vertical direction. The thrust force is neglected (Assumption 7.2.1.1).

\subsubsection{Geometric Compatibility}

Since the piston area is constant, the relationship between the piston height and the control volume is linear. It is evident from the geometry of Figure 7-1 that:

$$
V=A\left(Y_{\infty}+y\right)
$$

\subsubsection{Energy Balance Applied to the Control Volume}

To complete the problem, a force (pressure) versus deflection relationship must be derived from thermodynamic considerations. The first law of thermodynamics may be applied to the control volume shown in Figure $7-1$ as follows: 
change in stored energy $=$ energy in - energy out + work in + heat in

Based upon Assumptions 7.2.1.2 and 7.2.1.3 the heat in is zero and the change in potential energy of the gas flowing through the cylinder is zero. The energy balance then becomes:

$$
\frac{d U}{d t}-\left(h_{i} w_{i}-h_{0} w_{0}\right\}+P \frac{d V}{d t}=0
$$

The conservation-of-mass law may be applied to the control volume. The resulting equation equates the change of mass of the gas in the control volume to the difference between in flow and out flow. The results are:

$$
\frac{d w}{d t}=w_{i}-w_{0}
$$

The application of Assumption 7.2.1.4 gives:

$$
h_{\mathbf{i}}=h_{\mathbf{o}}
$$

For a perfect gas (Assumption 7.2.1.5), internal energy (u) and enthalpy (h) can be represented as:

$$
\begin{aligned}
& u=W u \\
& d u=C_{v} d T \\
& h=u+\frac{P V}{W}
\end{aligned}
$$

Substituting Equations (7-4) through (7-8) in Equation (7-3) and dividing the resulting equation by $C_{V} W T$, gives the following results: 


$$
\frac{d T}{T}=\frac{P V}{C_{v} W T} \frac{d W}{W}-\frac{P}{C_{v} W T} d V
$$

The perfect gas law and the specific heat definition gives the following relationships:

$$
\begin{aligned}
& \frac{P V}{W T}=\ddot{R} \\
& \frac{\overparen{R}}{C_{V}}=k-1
\end{aligned}
$$

Combining Equations (7-10) and (7-11) yields:

$$
\frac{P V}{W \bar{R} C_{v}}=k-1
$$

The substitution of Equations (7-10), (7-11), and (7-12) in Equation (7-9) yields:

$$
\frac{d T}{T}=(k-1) \frac{d W}{W}-(k-1) \frac{d V}{V}
$$

7.2.5 Perfect Gas Law Applied to the Control Volume .

The temperature variable in Equation (7-13) may be eliminated by introducing the perfect gas law. Written in logarithmic form, the perfect gas law is:

$$
\ln P+\ln V=\ln W+\ln \bar{R}+\ln T
$$


Differentiation of Equation (7-14) gives:

$$
\frac{d P}{P}+\frac{d V}{V}=\frac{d W}{W}+\frac{d T}{T}
$$

The combination of Equations (7.13) and (7-15) allows the elimination of the temperature variable. The result is:

$$
\frac{d P}{P}=k \frac{d W}{W}-k \frac{d V}{V}
$$

Expressing Equation (7-16) as a time rate equation gives:

$$
\frac{d P}{d t}=P\left(\frac{k}{W} \frac{d W}{d t}-\frac{k}{V} \frac{d V}{d t}\right)
$$

Equation (7-17) predicts the timo rate of change of the prossure within the control volume as a function of the weight and volume change.

7.2.6 Continuity and Energy Principles Involving

Gas Flow from the Control Volume

The first law equation (7-17) introduced a new variable: W. A flow equation is needed to express the mass change in the control volume witl respect to time. Such a relationship was derived in Section 7.2.4. The rcsulting equation was:

$$
\frac{d w}{d t}=w_{i}-w_{0}
$$


The exhaust flow through the orifice may be found by applying the continuity principle to the exhaust plane of the nozzle. The result is:

$$
w_{0}=\rho_{0} v_{0} a_{j} C_{x}
$$

The velocity $v_{0}$ and density $\rho_{0}$ of the exhaust gas at the nozzle throat may be determined from isentropic flow relationships. The results are:

$$
\begin{aligned}
& v_{0}=\sqrt{2 \frac{P V}{W}\left(1-P_{a} / P l^{\frac{k-1}{K}}\right.} \\
& \rho_{0}=\rho\left(P_{a} / P\right)^{1 / k}
\end{aligned}
$$

These results may be substituted into Equation (7-19) to predict the exhaust flow from the orifice. However, for small pressure differences across the exhaust nozzle, the compressibility of the gas may be neglected. In the present investigation, pressure differences of less than two pounds per square inch are involved. Consequently, the Assumption (7.2.1.6) of incompressible subsonic flow in the exhaust nozzle was made. The static pressure and total pressure of the gas in the control volume were assumed to be equal (Assumption 7.2.1.7).

For incompressible flow, the velocity at the exhaust exit plane is:

$$
v_{0}=\sqrt{\frac{2 g_{0}}{\rho}\left(P-P_{a}\right)}
$$

Combining Equations (7-18) and (7-19) gives:

$$
w_{0}=\sqrt{2 g_{0} \frac{W}{V}\left(P-P_{a}\right)} a_{j} C_{x}
$$


Equation (7-21) predicts the flow from an exhaust nozzle for small pressure difforences across the nozzle. For large pressure difference ratios (pressurc: ratios le:s than $0.9)$, Equation (7-21) should be modified to account for compressibility.

The weight flow into the control volume was assumed to $b:$ constant. The resulting relationship is:

$$
w_{\mathbf{i}}=\rho \mathbf{Q}_{\mathbf{i}}
$$

Combining Equations $(7 \cdot 4),(7 \cdot 20)$ and $(7 \cdot 21)$ gives:

$$
\frac{d W}{d t}=Q_{i} \rho-\sqrt{2 g_{0} \frac{W}{V}\left(P-P_{a}\right)} a_{j} C_{x}
$$

\subsubsection{Summary of Equations}

The system of equations which describes the simple dynamic model may be summarized as follows:

Definition of velocity

$$
\frac{d y}{d t}=\dot{y}
$$

Newton's second law:

$$
\frac{d \dot{y}}{d t}=-g+\frac{A}{W_{A}} g_{O}\left(P-P_{a}\right)
$$

First law of thermodynamics:

$$
\frac{d P}{d t}=P\left(\frac{k}{W} \frac{d W}{d t}-\frac{k}{V} \frac{d V}{d t}\right)
$$


Conservation of mass:

$$
\frac{d W}{d t}=\rho O_{i}-\sqrt{2 g_{0} \frac{W}{V}\left(P-P_{a}\right)} a_{j} C_{x}
$$

Geometric cpmpatibility:

$$
V=A\left(Y_{\infty}+y\right)
$$

The above set of linear, first order, differential equations may be solved by numerical procedures using the Runge and Kutta algorithm. ${ }^{\text {(47) }}$

\subsection{Air Cushion Trunk Dynanic Analysis}

\subsubsection{Approach and Assumptions}

The simplifind analysis developed in Section 7.2 may be applied to the Air Cushion Landing Syste $n$ by the introduction of a few complications. The performance of the trunk alone is considered in this section. Under this condition, cushion pressure is not allowed to build up beneath the fuselage. The configuration for the analysis is shown in Figure 7.2. The assumptions made in Section 7.2.1 are modified as follows:

\subsubsection{1 $\quad p_{c} / p_{j}=0$}

7.3.1.2 Only vertical motion is considered.

7.3.1.3 Thrust from the exhaust gas is included.

7.3.1.4 Elasticity of the trunk material is neglected. 


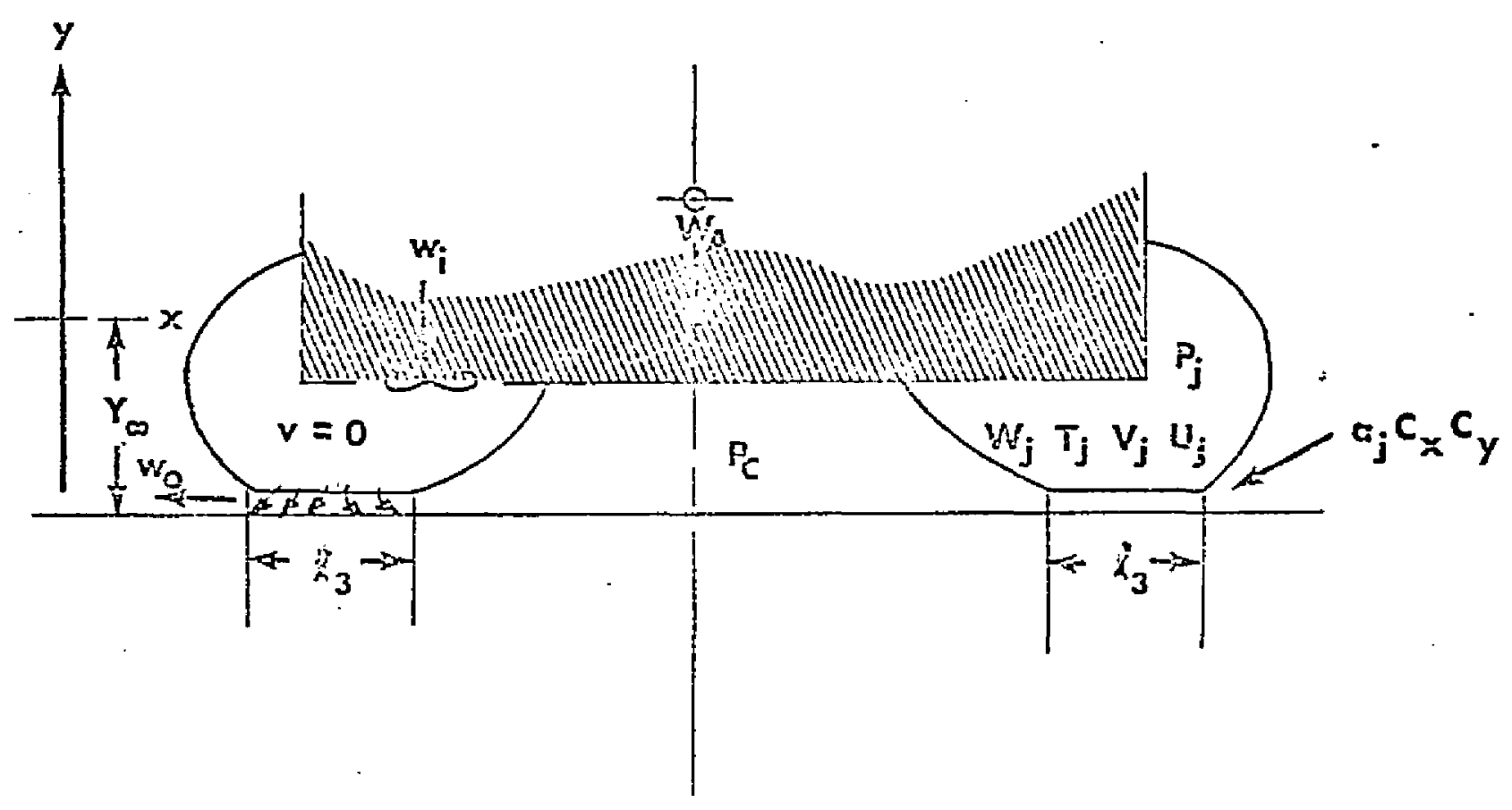

MODEL FOR TRUNK DYNAMIC ANALYSIS

FIGURE 7-2 
7.3.1.5 The flow in, $Q_{i}$, is a known function of $P_{j}$.

7.3.1.6 The distance above the ground at which the trunk begins to influence the dynamic response of the aircraft is designated $Y$. More precisely, $Y_{\infty}$ is the point above the ground at which $A_{3}=0$.

7.3.1.7 The coordinate system is selected as shown in Figure 7.2 such that $y=$ 0 at distance $Y_{\infty}$ above the ground. With this selection, the following relationship holds: $Y_{0}=-y$ for all $y \leqslant 0$.

7.3.1.8 The fan speed is assumed to be constant.

It may be noted that there are five major differences between the simple model of Section 1.1 and that of the air cushion trunk. These differences are as follows:

(1) The trunk model has a thrust force acting upward due to the change in momentum of the exhaust gas.

(2) The footprint (piston) area $\left(A_{3}\right)$ is not a constant, but is a function of y.

(3) The control volume is a nonlinear function of $Y$ rather than a simple linear function.

(4) The effective area of discharge of the orifice is not a constant, but is a function of $y$.

(5) The flow from the fan $\left(Q_{i}\right)$ is not a constant, but rather a function of trunk pressure $\left(\mathrm{p}_{\mathrm{j}}\right)$. 
Corrections have been incorporated in the simple model analysis to compensate for the differences listed above. These corrections are summarized in the discussion to follow.

\subsubsection{Correction for Thrust}

The thrust may be incleded by applying Newton's second law to the free body shown in Figure 7-2. The result is:

$$
\frac{W_{A}}{g_{o}} \frac{d^{2} y}{d t^{2}}=-W_{A} \frac{g}{g_{o}}+A_{3}\left(P_{j}-P_{a}\right)+F_{j}
$$

The vertical thrust is equal to the rate of change in momentum in the y direction. By assuming the velocity of the gas in the trunk is negligible it is possible to write:

$$
F_{j}=|\dot{m} v|_{y}=\frac{\rho}{g_{0}} Q_{j} v_{j} C_{z}=\frac{\rho}{g_{0}} v_{j}^{2} a_{j} c_{x} c_{y} C_{z}
$$

where:

$$
\begin{aligned}
c_{x}= & \text { coefficient of discharge for the trunk orifices } \\
c_{y}= & \text { coefficient to compensate for the dependence of the discharge } \\
& \text { cuefficient on } y, \text { and } \\
c_{z} \quad= & \text { coefficient to compensate for the various orifice angles. } \\
& \text { (Not all of the exhaust velocity is in the vertical direction.) }
\end{aligned}
$$

Values of these coefficients are determined in Sections 8.2, 8.4, and 8.3, respectively. The expression for velocity, Equation (7.19), may be substituted into Equation (7-25) to give: 


$$
F_{j}=2\left(P_{j}-P_{a}\right) a_{j} C_{x} C_{y} C_{z}
$$

Equation (7-26) may be substituted into Equation (7-24) to give:

$$
\frac{W_{A}}{g_{o}} \frac{d^{2} y}{d t^{2}}=-W_{A} \frac{g}{g_{o}}+A_{3}\left(P_{j}-P_{a}\right)+2\left(P_{j}-P_{a}\right) a_{j} C_{x} C_{y} C_{z}
$$

Equation (7.27) equates the sum of the vertical forces on the aircraft (weight, footprint pressure and thrust) to the product of the aircraft mass and the vertical acceleration. This equation provicies the required correction for jet thrust.

\subsubsection{Correction for Footprint Area}

The footprint area $\left(A_{3}\right)$ may be determined analytically from the values of footprint length $\left(\ell_{3}\right)$ predicted by the computer program developed in Section 4.5 . It was noted in Section 4.5 that $\ell_{3}$ is dependent on the trunk length $l$, the attachment points (a,b) and on $\mathrm{P}_{\mathrm{c}} / \mathrm{P}_{\mathrm{j}}$ and $Y_{\mathrm{o}}$. It is evident from Figure $4-8$ that different sections of the trunk on an actual model have different attachment points and trunk lengths. However, it is possible to separate the trunk into a number of segments which have approximately the same trunk length and attachment points. If the effective length of the $n^{\text {th }}$ segment is $L_{n}$ and there are a total of $m$ segments, the total footprint area is:

$$
A_{3}=\sum\left(l_{3}\right)_{n} L_{n}
$$

Equation (7.28) predicts the total footprint area of the trunk as the sum of the footprint areas of all the trunk segments. The footprint length $\ell_{3}$ is a known function of $Y_{o}$ and $p_{c} / p_{j}$. For the case considered in this section, $p_{c} / p_{j}=0$. The variation of $\ell_{3}$ with 
$Y_{0}$ for $p_{c} / p_{j}=0$ was shown in Figures 4-21 and 4.22 . The former figure is for a side trunk segment and the latter is for an end trunk segment.

The value of $L_{n}$ is a constant for straight trunk segments such as the side segment shown in Figure 4-8. However, for curved segments such as the end segment shown in Figure 4-8, $L_{n}$ is dependent on $P_{c} / p_{j}$ and $Y_{0}$. This dependence may be calculated from the computer progran given in Appendix III. Using the above procedure, it is possible to determine $A_{3}$ as a function of $Y_{0}$ for the trunk on a given model.

\subsubsection{Correction for Trunk Volume Change}

The trunk volume $\left(\mathrm{V}_{\mathrm{j}}\right)$ may be determined analytically from the values of cross-section area $\left(A_{j}\right)$ predicted by the computer program developed in Sections 4.5 and 4.6. The trunk may be divided into a number of segments in a manner similar io that described in Suction 7.3.3. If the effective length of the $n^{\text {th }}$ segment is $K_{n}$ and thero are a total of m segments, the total trunk volume is:

$$
v_{j}=\sum\left(A_{j}\right)_{n} K_{n}
$$

The trunk segment cross-sectional area $\left(A_{j}\right)$ is a known function of $Y_{o}$ and $P_{c} / P_{j}$. The variation of $A_{j}$ with $Y_{o}$ for $p_{c} / P_{j}=0$ was shown in Figures 4-23 and 4.24. The former figure is for a side trunk segment and the latter is for an end trunk segment.

The value of $K_{n}$ is constant for straight trunk segments such as the side trunk segment shown in Figure 4-8. However, the end trunk segment is a volume of revolution. For a volume of revolution, the effect length may be defined as follows:

$$
K_{n}=\bar{r}_{n} a_{n}
$$


where

$$
\begin{aligned}
\vec{r}_{n} \quad= & \text { radius from the center of revolution to the centroid of the area } \\
& A_{j} \text { for the } n^{\text {th }} \text { trunk segment } \\
a_{n} \quad= & \text { angle of revolution for the volume of the } n^{\text {th }} \text { trunk segment }
\end{aligned}
$$

The values of $\overline{r_{n}}$ and $a_{n}$ may be calculated from the geometry of the particular model and trunk seyment.

Using the above procedure it is possible to determine $A_{j}$ as a function of $Y_{0}$ for the trunk on a given model.

\subsubsection{Correction for Variable Discharge Area}

As the trunk is pressed against the ground, the flow from trunk exhaust orifices in the footprint area is reduced. A discharge coefficient, $C_{y}$, has been introduced to account for the resulting dependence of the trunk exhaust flow on the vehicle height $\left(Y_{0}\right)$.

The resulting flow relationship is:

$$
a_{j}=\sqrt{\frac{2 g_{o}}{\rho}\left(p_{j}\right)} a_{j} c_{x} c_{y}
$$

where $C_{y}$ is a function of $Y_{0}$.

The value of $C_{y}$ is determined by computing the flow from the various trunk segments $l_{1}, \ell_{2}$, and $\ell_{3}$ shown in Figure 4-2. The resulting flows are designated $a_{1}, Q_{2}$, and $\mathrm{Q}_{3}$, and may be computed as follows:

$$
\mathrm{o}_{1}=\sqrt{\frac{2 \mathrm{~g}_{\mathrm{o}}}{\rho}\left(\mathrm{p}_{\mathrm{j}}\right)} \mathrm{a}_{1} \mathrm{c}_{x}
$$




$$
\begin{aligned}
& \mathrm{a}_{2}=\sqrt{\frac{2 \mathrm{~g}_{0}}{\rho}\left(\mathrm{p}_{\mathrm{j}}\right)} \mathrm{a}_{2} \mathrm{c}_{\mathrm{x}} \\
& \mathrm{a}_{3}=\sqrt{\frac{2 \mathrm{~g}_{0}}{\rho}\left(\mathrm{p}_{\mathrm{j}}\right)} \mathrm{a}_{3} \mathrm{c}_{\mathrm{x}} \\
& \mathrm{a}_{\mathrm{j}}=\mathrm{a}_{1}+\mathrm{a}_{2}+\mathrm{a}_{3}
\end{aligned}
$$

The values of $a_{1}$ and $a_{2}$ are determined by the total trunk orifice area in segments $\ell_{1}$ and $\ell_{2}$ respectively. The value of $a_{3}$ ' is determined by the area which controls the flow from trunk segment $\ell_{3}$.

If the area between the trunk and the ground is less than the trunk orifice area $a_{3}$, then flow is controlled by the ground clcarance rather than by the trunk area, Consequently, the effective flow area for segment $l_{3}$ may be written:

$$
a_{3}^{\prime}=\left\{\begin{array}{c}
a_{3} \\
2 s_{3} d \frac{c_{D}}{c_{x}}
\end{array}\right\} \quad \text { whichever is smaller }
$$

The value of $a_{3}$ may be approximated by the product of the footprint area $\left(A_{3}\right)$ and the porosity of the trunk $\xi$ in the footprint area. The result is:

$$
a_{3}=A_{3} \xi
$$

The porosity of the trunk $\xi$ is defined as the ratio of orifice area to total area in the section of the trunk containing the orifices. 
The total jet area of the trunk is the sum of the area in the three segments.

$$
a_{j}=a_{1}+a_{2}+a_{3}
$$

An expression for $C_{y}$ is obtained by combining Equations (7-31), (7-32), (7-33), (7-34), and (7-35). The result is:

$$
c_{y}=\frac{a_{1}+a_{2}+a_{3}}{a_{j}}
$$

It is evident from Equation (7-39) that $C_{y}=1.0$ whenever $a_{3}{ }^{\prime}=a_{3}$.

Th: equation for $\mathrm{C}_{\mathrm{y}}$ may be further simplified by substituting Equations (7.37) and (7-38) inio (7-39). The result is:

$$
c_{y}=\frac{a_{j}-A_{3} \xi+a_{3}}{a_{j}}
$$

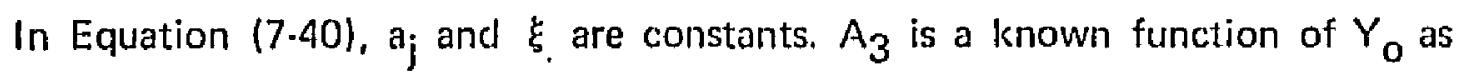
developed in Section 7.3.3. The value of $a_{3}$ was defined as follows:

$$
a_{3}^{\prime}=\left\{\begin{array}{c}
A_{3} \xi \\
2 s_{3} d c_{D} / c_{x}
\end{array}\right\} \quad \text { whichever is less }
$$

The values of $S_{3}, C_{X}$, and $C_{D}$ are constants. The value of $d$ is dependent on a number of variables including $Y_{0}$. An assessment of the value of $d$ is presented in the remainder of this section. 
An estimate of the jet height $d$ variation with $Y_{0}$ has been made based upon an analysis, conducted by $\operatorname{Han}^{\prime} 49$ ), of idealized flow in a channel with injection from a porous wall. In his analysis, Han determined the pressure distribution in a channel of the configuration shown in Figurc 7-3. The independent variables for this analysis were $d, \ell_{3}$, $P_{j}$, and $\xi$. The latter quantity is the effective wall porosity and may be expressed by the ratio $a_{3} / A_{3}$. The total vertical force per unit length which is developed in the footprint arca can be determined by integrating the pressure over the footprint length. Using a trurk pressure of $80 \mathrm{psf}$ and the porosity value for the model side trunk given in Table 5.1, the footprint force was determined as a function of jot height and footprint length. The results are plotted in Figure 7-4.

Figure 7-4 presents the load-deflection characteristics of the jet for various footprint lengths. The actual jet height is determined by the load which the jet must support for a given trunk configuration.

A free body diagram of a trunk configuration is shown in Figure 7-5. Force equilibrium applied in the $y$ direction gives:

$$
p_{j} l_{3}-F_{3}-2 T_{t} \sin \psi_{t}=0
$$

The value of $T_{t}$ was given by Equation (4-1).

$$
T_{t}=R_{\mathfrak{i}} p_{j}
$$

Combining Equations (4-41) and (4-1) gives:

$$
F_{3}=p_{j}\left(\ell_{3}-2 R_{1} \sin \psi_{t}\right)
$$



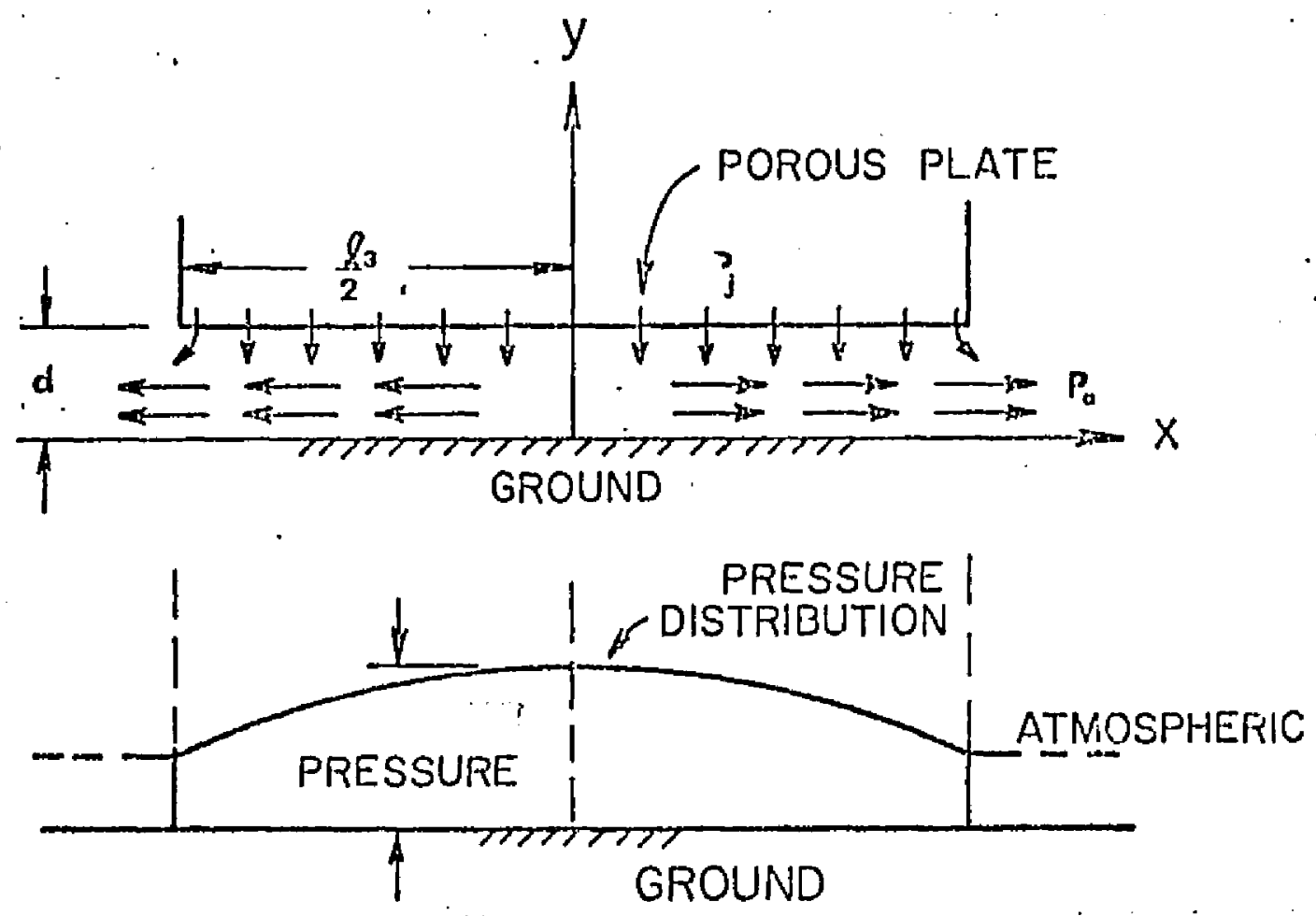

MODEL FOR PRESSURE DISTRIBUTION

ACROSS THE FOORPRINT

FIGURE 7-3 


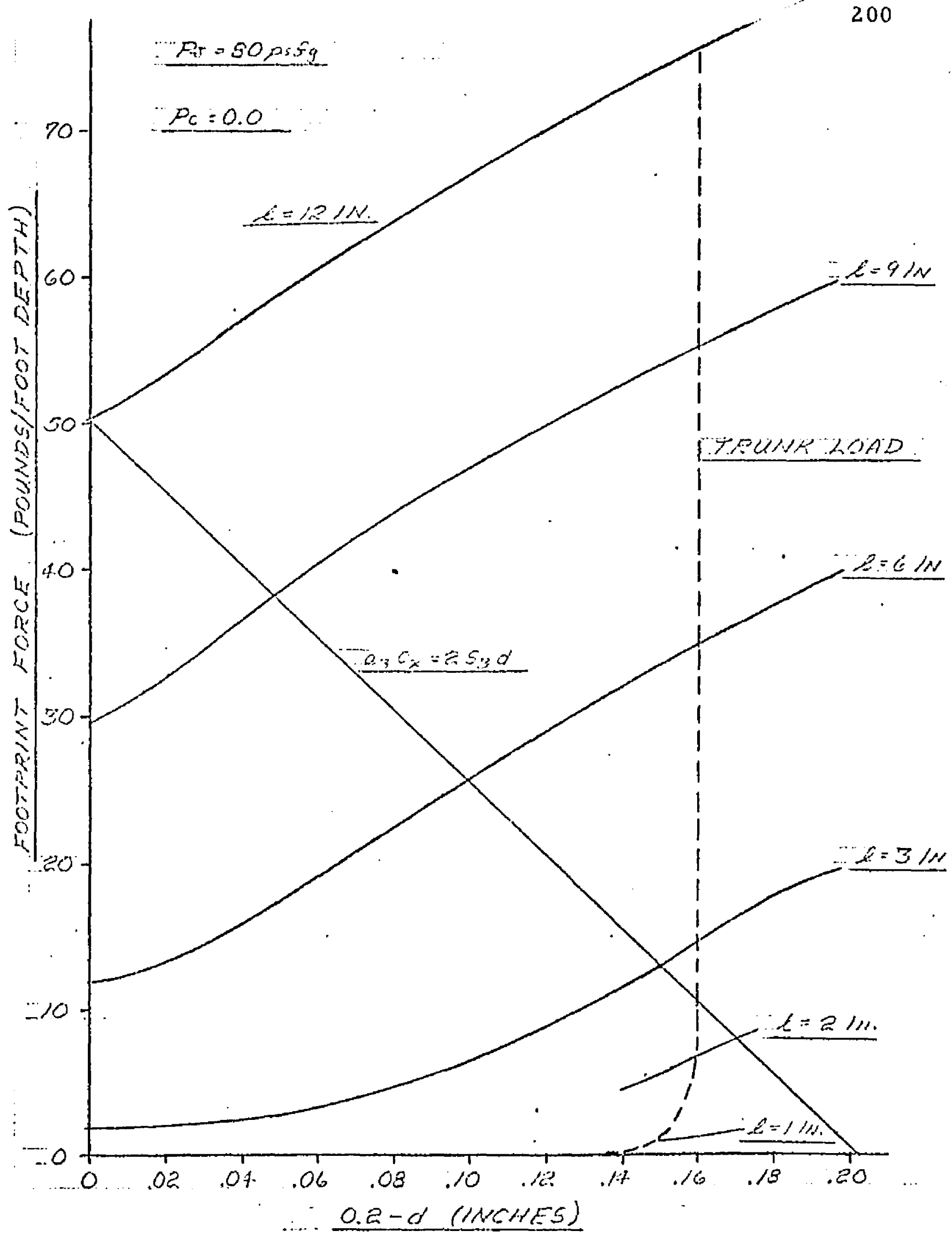

LOAD-DEFLECTION CHARACTERISTICS

OF THE CUSHION EXHAUST GAP

FIGURE 7-4 


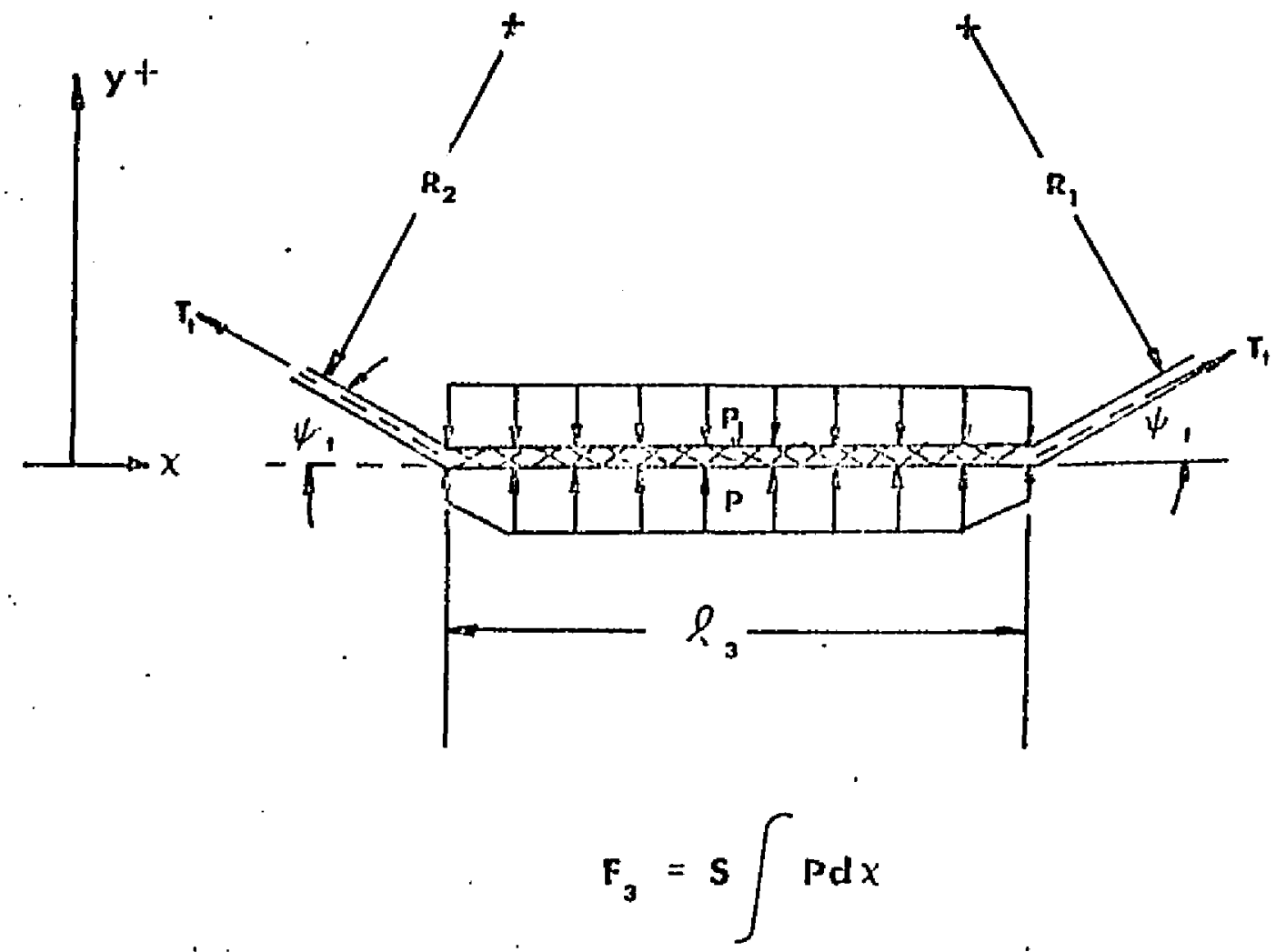

FREE BODY DIAGRAM FOR TRUNK FOOTPRINT - figure 7-5 
For the analysis of the trunk shape presented in Section $4.5, \psi_{\mathrm{t}}$ was assumed to be zero. However, this analysis was made for a trunk section with free edges. A trunk on a three-dimensional model is constrained by the curvature of the trunk in the peripheral direction. Consequently, it is possible for a finite angle to exist at the edge of the footprint. Such an angle has been observed on a three-dimensional model. A value of $\psi_{t}=4^{\circ}$ constant gives reasonable agreement with observed results on the dynamic model. Using the assumed value of $\psi_{t}$, the values of $\mathrm{F}_{3}$ computed from Equation (7.42) are shown as the load line on Figure 7.4.

The jet heights at which $a_{3}=2 S_{3}$ d are also shown on the curve. From the results presented in Figure 7-4, it is assumed that $d=$ constant for values of $\ell_{3}$ greater than about 2 inches.

\subsubsection{Correction for Flow from the Fan}

The flow from the fan is dependent upon the fan speed and the exhaust pressure. This variation may be delermined by standard fan caljbration tests. Such a test is described in Section 8.6 and the test results are shown in Figure 8.3.

For the purposes of this analysis, the fan speed is assumed to be constant during landing impact (Assumption 7.3.1.7).

\subsubsection{Summary of Equations}

The changes required to apply the system of equations developed in Section 7.2 to the air cushion trunk system have been developed in Sections 7.3.1 through 7.3.6. The resulting equations may be summarized as follows:

\section{Definition of velocity}

$$
\frac{d y}{d t}=\dot{y}
$$


Newton's second law

$$
\frac{d \dot{y}}{d t}=\frac{g_{o}}{W_{A}}\left[-W_{A} \frac{g}{g_{O}}+A_{3}\left(P_{j}-P_{a}\right)+2\left(P_{j}-P_{a}\right) a_{j} C_{x} C_{y} C_{z}\right]
$$

First law of thermodynamics

$$
\frac{d P_{j}}{d t}=p_{j}\left(\frac{k}{w_{j}} \frac{d W_{j}}{d t}-\frac{k}{V_{j}} \frac{d V_{j}}{d t}\right)
$$

Conservation of mass

$$
\frac{d W_{j}}{d t}=\rho Q_{i}-\sqrt{2 g_{0} \frac{W_{j}}{V_{j}}\left(P_{j}-P_{a}\right)} a_{j} c_{x} c_{y}
$$

Geometric compatibility

$$
v_{j}=\sum\left(A_{j}\right)_{n} K_{n}
$$

In the system of equations, there are five dependent variables: $\dot{y}, y_{,} P_{j}, V_{j}$, and $W_{j}$. The following variables are known and constant: $W_{A}, g_{0}, P_{a}, a_{j}, C_{x}, C_{2}, k, \rho, K_{n}, g$. The following variables are known functions of the dependent variables:

$$
\begin{aligned}
& A_{3}=f\left(Y_{0}\right) \text { as developed in Section 7.3.3. } \\
& A_{j}=f\left(Y_{0}\right) \text { as developed in Section 7.3.4. } \\
& C_{y}=f\left(Y_{0}\right) \text { as developed in Section 7.3.5. } \\
& a_{i}=f\left(P_{j}\right) \text { as developed in Section 7.3.6. }
\end{aligned}
$$



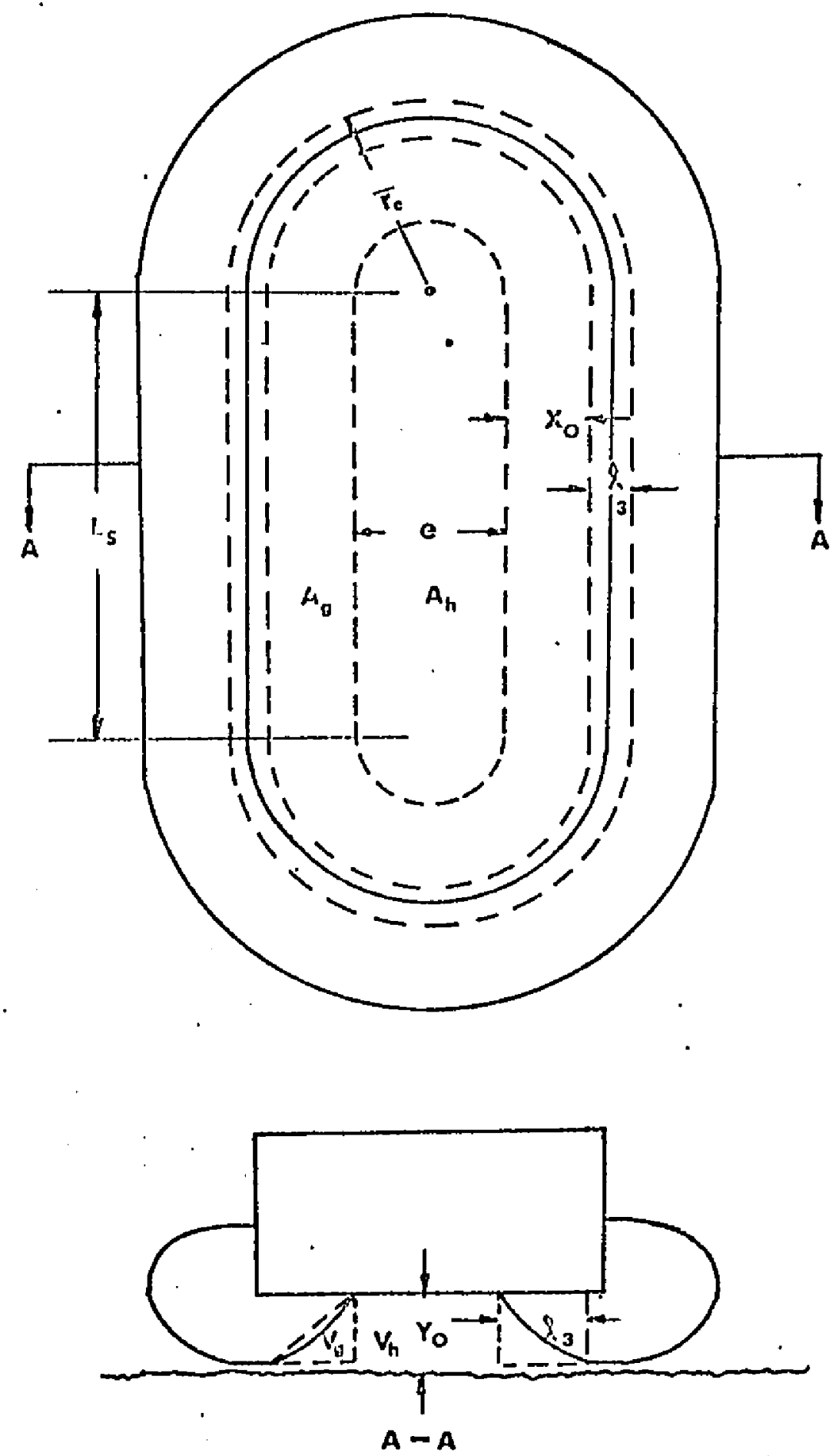

MODEL FOR AIR CUSHIOH SYSTEM DYNAMIC ANALYSIS FIGURE 7-6 
The system of equations and functions described in this section has been programmed and solved on a digital computer using the Runge and Kutta algorithm. (47) The computer results have been compared with experimental results in Chapter 8.

\subsection{Complete Air Cushion System Dynamic Analysis}

\subsubsection{Approach and Assumptions}

The analysis developed in Sections 7.2 and 7.3 may be applied to the complete air cushion system by introducing relationships to account for the effect of cushion pressure on the system response. The configuration for the analysis is shown in Figure 7.6. The assumptions made in Section 7.3.1 are modified as follows:

7.4.1.1 The cushion pressure is allowed to build up so that $\mathrm{p}_{\mathrm{c}} / \mathrm{P}_{\mathrm{j}} \neq 0$.

7.4.1.2 The model is of the type shown in Figure 7-6. The trunk cross section is the same at any section.

7.4.1.3 The trunk configuration is identical to the side trunk whose properties were listed in Table 5.1.

A number of additional simplifying assumptions are included in the sections to follow.

The equations of motion developed in Section 7.3 may be applied to a complete cushion-trunk system by the introduction of corrections for cushion pressure.

- The necessary corrections are as follows:

Correction of the second law equation for the reaction force from the cushion pressure. 
(2) Prediction of the area over which the cushion pressure acts.

(3) Prediction of the cushion pressure.

(4) Prediction of the cushion volume.

(5) Prediction of the cushion flow.

(6) Prediction of the influence of cushion pressure on trunk flow.

(7) Prediction of the influence of cushion pressure on trunk footprint area.

(8) Prediction of the influence of cushion pressure on trunk volume.

(9) Prediction of the influence of cushion pressure on vertical thrust.

These corrections have been developed in the sections to follow.

\subsubsection{Cushion Reaction}

-The cushion pressure reaction may be included in the second law equation, Equation (7-27), by the introduction of an additional force term. The resulting equation is:

$$
\frac{W_{A}}{g_{0}} \frac{d^{2} y}{d t^{2}}=-W_{A} \frac{g}{g_{o}}+A_{3}\left(P_{j}-P_{a}\right)+A_{c}\left(P_{c}-P_{a}\right)+F_{j}
$$


Equation (7.45) equates the sum of the vertical forces on the aircraft (weight, footprint force, cushion force and thrust) to the product of the mass and the vertical acceleration.

\subsubsection{Cushion Support Area}

The cushion support area $\left(A_{c}\right)$ is a function of both $Y_{o}$ and $p_{c} / p_{j}$. Figure 7.6 shows that the cushion area may be divided into two parts $-A_{h}$ and $A_{g}$. The $A_{h}$ part is the area under the hard structure which is enclosed by the inner trunk attacloment. This area is constant. The $A_{g}$ part is the area between the inner trunk attachment and the inner ground tangent. This area is dependent on the width $x_{o}$ and the effective length $S_{g}$. The total cushion area may be written as the sum of the parts as follows:

$$
A_{c}=A_{h}+S_{g} X_{o}
$$

The value of $A_{h}$ is constant, and $S_{g}$ may be considered constant for small changes in $X_{0}$. The value of $X_{0}$ is dependent on $Y_{0}$ and $p_{c} / p_{j}$. The relationship between these variables has been determined for a straight section of trunk with unconstrained edges using the computer program described in Appendix 111. The results for the side trunk section are shown in Figure 7-7. The carpet plot in Figure 7-7 shows constant tines of $p_{c} / p_{j}$ and $\ell_{3}$.

It is evident from Figure 7-7 that for a given $\ell_{3}$, the trunk low point $x_{0}$ moves outward with increasing $\left(\mathrm{p}_{\mathrm{c}} / \mathrm{p}_{\mathrm{j}}\right)$ thereby increasing the cushion support area. On the other hand, it is evident that decreasing $Y_{0}$ at constant $p_{c} / p_{j}$ causes an increase in the footprint length $\left(\ell_{3}\right)$. The increase in footprint length, in turn, results in a decrease in $X_{0}$ and an attendant decrease in cushion support area.

During a landing impact, the energy absorption process starts at the point defined by $\ell_{3}=0$ and $p_{c} / p_{j}=0$. For the case when $p_{c}=0$, the process proceeds along the $p_{c} / p_{j}=0$ 
208

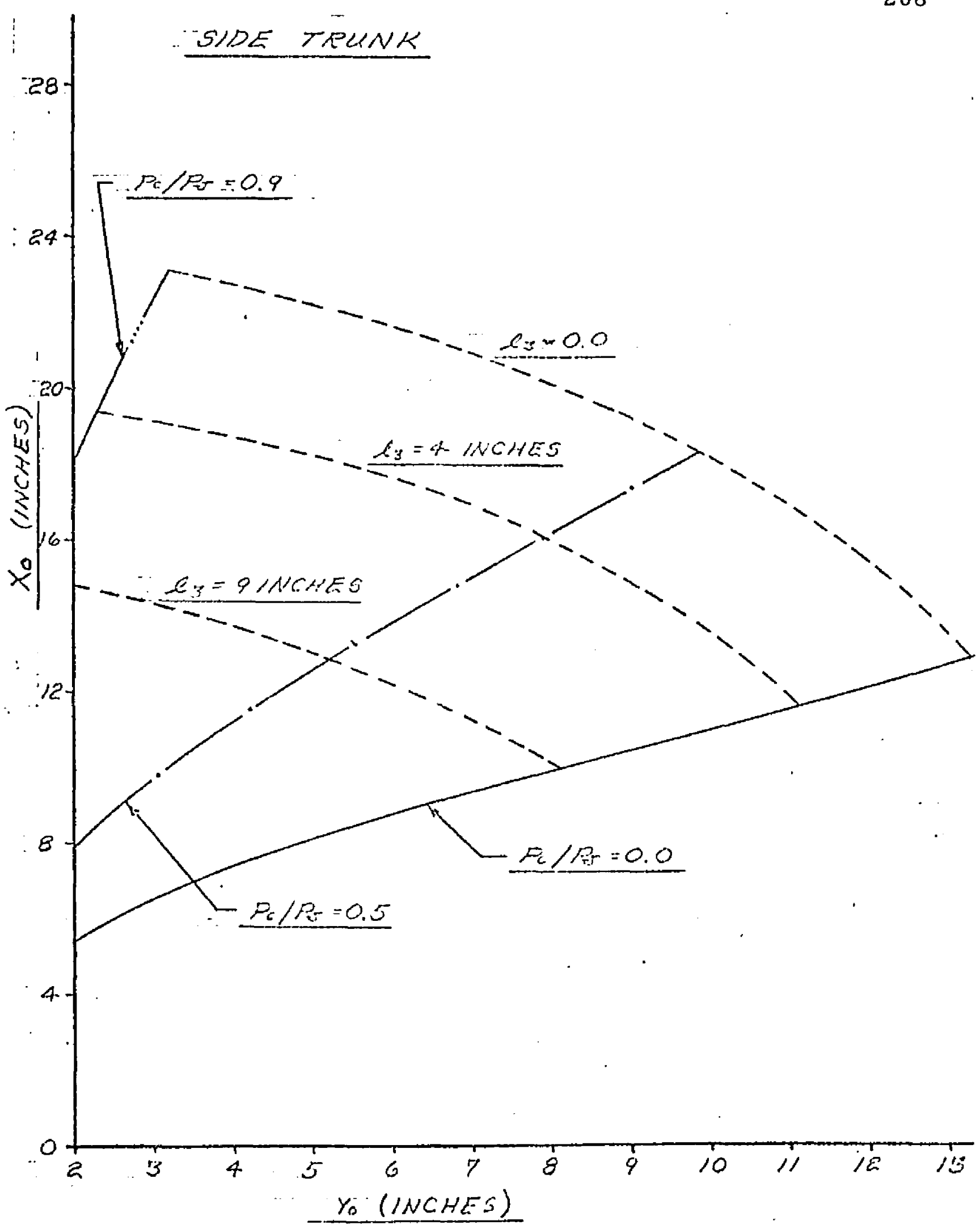

$$
\begin{gathered}
x_{0} \text { vS } y_{0} \text { FOR MODEL TRUNK } \\
\text { FIGURE } 7-7
\end{gathered}
$$


lines. On the other hand, for the case when the change in $Y_{0}$ is slow and the weight is supported only by the cushion pressure, the process follows the $\ell_{3}=0$ line. An actual impact process follows a path somewhere between these two extremes.

It should be noted that Figure 7.5 is for a trunk section with free ends. For a trunk on an actual model, there are no free ends. The trunk closes on itself as shown in Figure 7-6. In order for $x_{o}$ to increase with increasing $p_{c} / p_{j}$, the trunk must stretch along circumferential length $\mathrm{S}_{\mathrm{g}}$. The degree of constraint which results depends upon the elasticity of the material and the shape of the model. As a consequence, caution should be exercised in applying the free shape curves to an actual model. However, such curves are valuable in making approximations for the relationships among the variables.

In view of the offsetting influences of $p_{c} / p_{j}$ and $\ell_{3}$ on the value of $x_{0}$, a first approximation of $X_{0}=$ constant is reasonable for the trunk shape shown in Figure 7-6.

\subsubsection{Cushion Pressure Prediction}

The cushion pressure equation may be developed in a manner identical to that presented in Sections 7.2.4 and 7.2.5. The resulting equation is:

$$
\frac{d P_{c}}{d t}=P_{c}\left(\frac{k}{W_{c}} \frac{d W_{c}}{d t}-\frac{k}{V_{c}} \frac{d V_{c}}{d t}\right)
$$

Equation (7-47) predicts the cushion pressure change with time as a function of the change of volume and change in weight of the gas in the cushion. In order to predict the cushion pressure, it is necessary to predict the volume and weight change of the cushion air.

\subsubsection{Cushion Volume Prediction}

The cushion volume is a function of $Y_{0}, \ell_{3}$, and $p_{c} / p_{j}$. However, as in the case of the cushion support area, the influence of $p_{c} / p_{j}$ and $\ell_{3}$ tend to offset each other. 
The cushion volume on the air cushion model shown in Figure 7-6 is considered to be composed of two parts - the portion directly under the hard structure $\left(V_{h}\right)$ and the portion directly under the flexible trunk $\left(V_{g}\right)$.

The volume under the hard structure $\left(V_{h}\right)$ is a linear function of $Y_{0}$ and is independent of $\mathrm{p}_{\mathrm{C}} / \mathrm{p}_{\mathrm{j}}$. The equation for this portion of the volume is:

$$
V_{h}=A_{h} Y_{0}
$$

The volume under the trunk is more difficult to calculate. For the purposes of simplification, a triangular cross section of $V_{g}$ is assumed. Figure 7-6 shows that the altitude and base of the triangle have lengths of $\dot{Y}_{0}$ and $X_{0}$ respectively. If the triangular area is assumed to be constant around the trunk, the portion of the cushion volume under the trunk is computed as follows:

$$
v_{g}=\frac{1}{2} X_{o} Y_{0} S_{g}^{\prime}
$$

The variable $\mathrm{S}_{\mathrm{g}}$ is defined as the effective length for calculating the volume from the cross-sectional area. Figure 7.6 shows that the volume $V_{g}$ consists of straight sections along the sides. However, the two ends, taken together, form a volume of revolution. The effective length for the two side volumes is $2 \mathrm{~L}_{\mathrm{s}}$. The effective length for the end volumes is the distance from the center of rotation to the centroid of the triangular area times the angle of revolution. The resulting equation for $S_{g}^{\prime}$ is:

$$
\mathrm{S}_{\mathrm{g}}{ }^{\prime}=2 \mathrm{~L}_{\mathrm{s}}+2 \pi\left(\frac{\mathrm{e}}{2}+\frac{\mathrm{X}_{\mathrm{o}}}{3}\right)
$$

The relationship between $X_{0}$ and $Y_{0}$ was shown in Figure 7-5 and discussed in Section 7.4.3. As a first approximation, $X_{0}=$ constant is a reasonable assumption. 
Combining Equations (7-48), (7-49), and (7-50) gives the following equation for the cushion volume:

$$
v_{c}=\left\{A_{h}+X_{0}\left[L_{s}+\pi\left(\frac{e}{2}+\frac{x_{0}}{3}\right)\right]\right\} Y_{0}
$$

In Equation (7-51) the variables $A_{h}, L_{s}$ and $e$ are assumed constant. $A$ relationship of the type given in Figure 7-7 may be used to relate $X_{0}$ to $Y_{0}$. However, as a first approximation, $X_{0}=$ constant is assumed.

\subsubsection{Cushion Flow Prediction}

In a manner similar to the analysis developed in Section 7.2.6, the conservation of mass law may be written for the cushion:

$$
\frac{d w_{c}}{d t}=\left(w_{c}\right)_{i}-\left(w_{c}\right)_{0}
$$

All flow into the cushion cavity comes from the orifices in segment $\ell_{2}$ of the trunk. This segment is shown in Figure 4-2. The total area of orifices in segment $\ell_{2}$ is a $a_{2}$. The flow into the cushion from the trunk may be written:

$$
\left(w_{c}\right)_{i}=(\text { sign }) \sqrt{2 g_{0} p\left|\left(P_{j}-P_{c}\right)\right|} \quad a_{2} c_{x}=w_{2}
$$

The sign on the radical in Equation (7-53) takes the same sense as the quantity $\left(P_{j}-P_{c}\right)$. This convention is necessary because it is possible during dynamic impact for $P_{c}$ to exceed $P_{j}$. The direction of flow is, of course, from the higher pressure to the lower pressure. 
The value of $\mathrm{a}_{2}$ may be determined by summing the area of all the orifices in segment $\ell_{2}$. The total number of rows of orifices in segment $\ell_{2}$ is designated as $M_{2}$. Each row has an effective thickness $t_{n}$ and a length $s_{n}$. The total area $a_{2}$ is written:

$$
a_{2}=\sum \cdot t_{n} \dot{s}_{n}
$$

In Equation (7-55) the values of $s_{n}$ and $t_{n}$ are known constants. The value of $\mathrm{N}_{2}$ is dependent on $\mathrm{p}_{\mathrm{c}} / \mathrm{p}_{\mathrm{j}}$ and $\mathrm{Y}_{\mathrm{O}}$. This dependence has been determined using the computer program listed in Appendix III. The results are presented in Figure 7-8.

The flow out of the cushion is through the cushion exhaust nozzle. This flow may be expressed:

$$
\left(w_{c}\right)_{0}=\sqrt{2 g_{0} \rho\left(P_{C}-P_{a}\right)} s_{3} d C_{D}
$$

In Equation (7-55), $g_{0}, \rho, S_{3}$ and $C_{D}$ are assumed constant. The variation of $d$ is determined as discussed in Section 7.3.5.

An expression for the cuslion flow may be written by combining Equations (7-52), (7-53), and (7-55). The result is:

$$
\frac{d W_{c}}{d t}=w_{2}-\sqrt{2 g_{0} \rho\left(P_{c}-P_{a}\right)} s_{3} d C_{D}
$$

\subsubsection{Influence of Cushion Pressure on Trunk Flow}

The flow into the trunk is dependent only on trunk pressure and fan speed. No modification to the fan flow relationship is necessary to correct for the effect of cusinion pressure. 


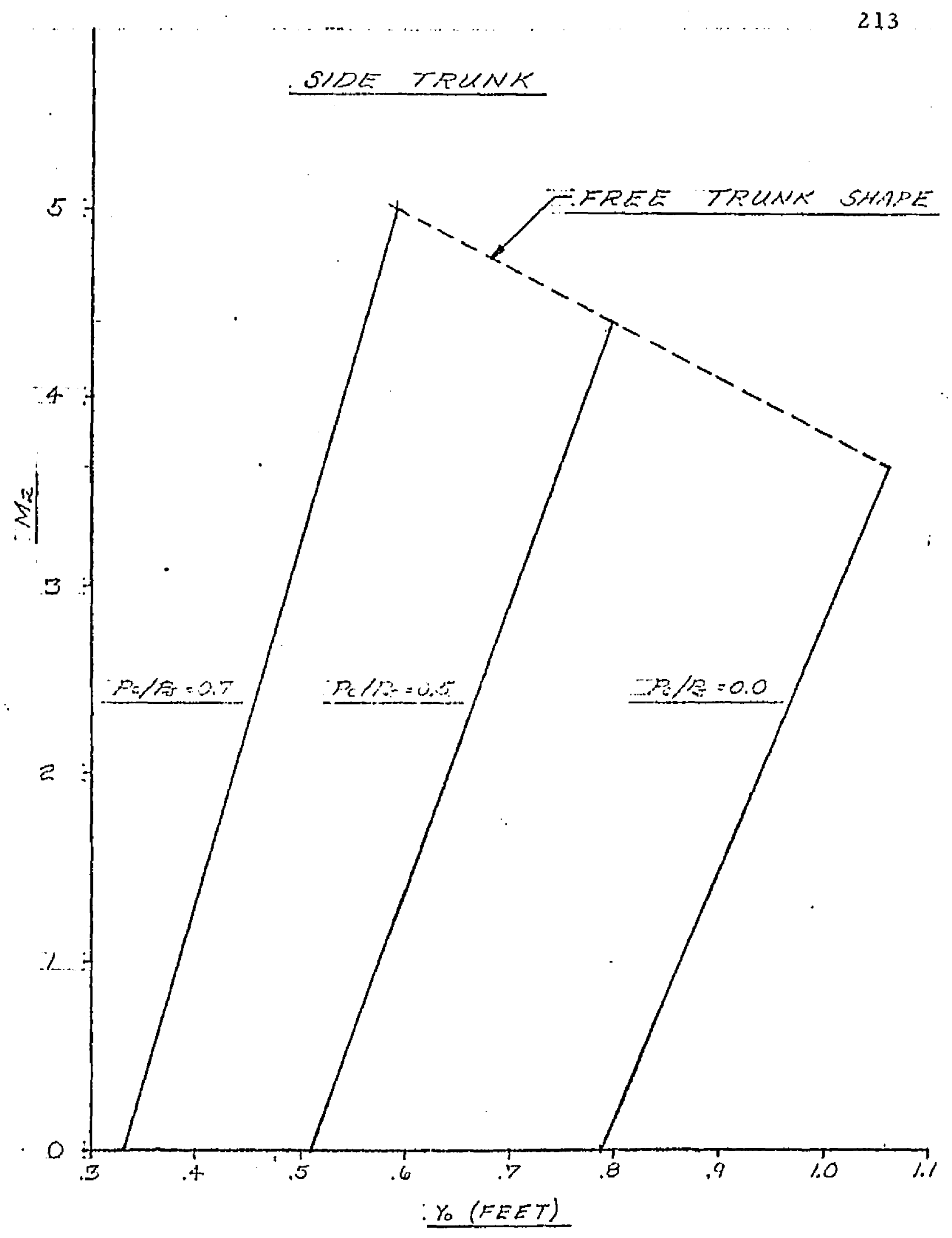

$M_{2}$ vs $Y_{0}$ FOR MODEL TRUNK

FIGURE $7 .-8$ 
The flow from the trunk is influenced by the cushion pressure. The nozzle in trunk segment $\ell_{2}$ exhausts to cushion pressure rather than atmospheric. The exhaust from the trunk segment $\ell_{3}$ expands to $P_{c}$ on the inside and $P_{a}$ on the outside.

The flow from the trunk may be written as the sum of the flow from the three segments.

$$
\left(w_{j}\right)_{0}=w_{1}+w_{2}+w_{3}
$$

The flow from segment $\ell_{1}$ exhausts to atmospheric pressure.

$$
w_{1}=\sqrt{2 g_{0} \rho\left(P_{j}-P_{a}\right)} a_{1} c_{x}
$$

The flow from segment $\ell_{2}$ exhausts to cushion pressure.

$$
w_{2}=\sqrt{2 g_{0} \rho\left|\left(P_{j}-P_{a}\right)\right|} a_{2} C_{x}
$$

The sign for $w_{2}$ is positive when $p_{j}>p_{c}$ and negative when $p_{c}<p_{j}$.

The flow from segment $\ell_{3}$ is assumed to exhaust to atmospheric pressure.

$$
w_{3}=\sqrt{2 g_{0} \rho\left(P_{j}-P_{a}\right)} a_{3}^{\prime} C_{x}
$$

The value of $\mathrm{a}_{3}{ }^{\prime}$ is determined by the area which controls the flow from the trunk segment $l_{3}$. The area $a_{3}$ may be expressed:

$$
a_{3^{\prime}}=\left\{\begin{array}{c}
A_{3}^{\xi} \\
s_{3} d \frac{C_{D}}{C_{x}}
\end{array}\right\} \quad \text { whichever is less }
$$


In Equations (7-58) through (7-60) the independent variables are $P_{j}$ and $P_{c}$. The variables $\mathrm{a}_{1}, \mathrm{a}_{2}$, and $\mathrm{a}_{3}$ are dependent on $\mathrm{Y}_{\mathrm{o}}$ and $\mathrm{p}_{\mathrm{c}} / \mathrm{p}_{\mathrm{j}}$. The value $\mathrm{a}_{1}$ may be determined by rearranging Eqquation (7-38).

$$
a_{1}=a_{j}-a_{2}-a_{3}
$$

whero $\mathbf{a}_{\mathbf{j}}$ is constant.

The values of $\mathrm{a}_{2}$ and $\mathrm{a}_{3}$ were determined in Sections 7.4 .6 and 7.3 .5 by Equations (7-54) and (7-37), respectively.

The value of $a_{3}$ is determined by the same method discussed in Section 7.3.5.

\subsubsection{Influence of Cushion Pressure on Trunk Footprint Area}

The influence of $\mathrm{p}_{\mathrm{c}} / \mathrm{p}_{\mathrm{j}}$ on trunk footprint length for a side trunk section with free edges is shown in Figure 4-21. For the $p_{c}=0$ case, the relationship between $\ell_{3}$ and $Y_{0}$ is given by the $\mathrm{p}_{\mathrm{c}} / \mathrm{p}_{\mathrm{j}}=0$ curve. Higher values of $\mathrm{p}_{\mathrm{c}} / \mathrm{p}_{\mathrm{j}}$ tend to decrease $\ell_{3}$ for a given $Y_{0}$.

The total footprint area of the model shown in Figure 7-6 may be computed as the sum of the area of the side sections and the area of the end sections. The resulting equation is:

$$
A_{3}=L_{s} l_{3}+\pi\left[\left(\frac{e}{2}+x_{0}+l_{3}\right)^{2}-\left(\frac{e}{2}+x_{0}\right)^{2}\right]
$$

In Equation (7.62), $\mathrm{I}_{\mathrm{s}}$ and $\mathrm{e}$ are constants. As a first approximation, $X_{0}$ is assumed constant. The variation of $\ell_{3}$ with $Y_{0}$ and $p_{c} / p_{j}$ is given in Figure 4-21. 


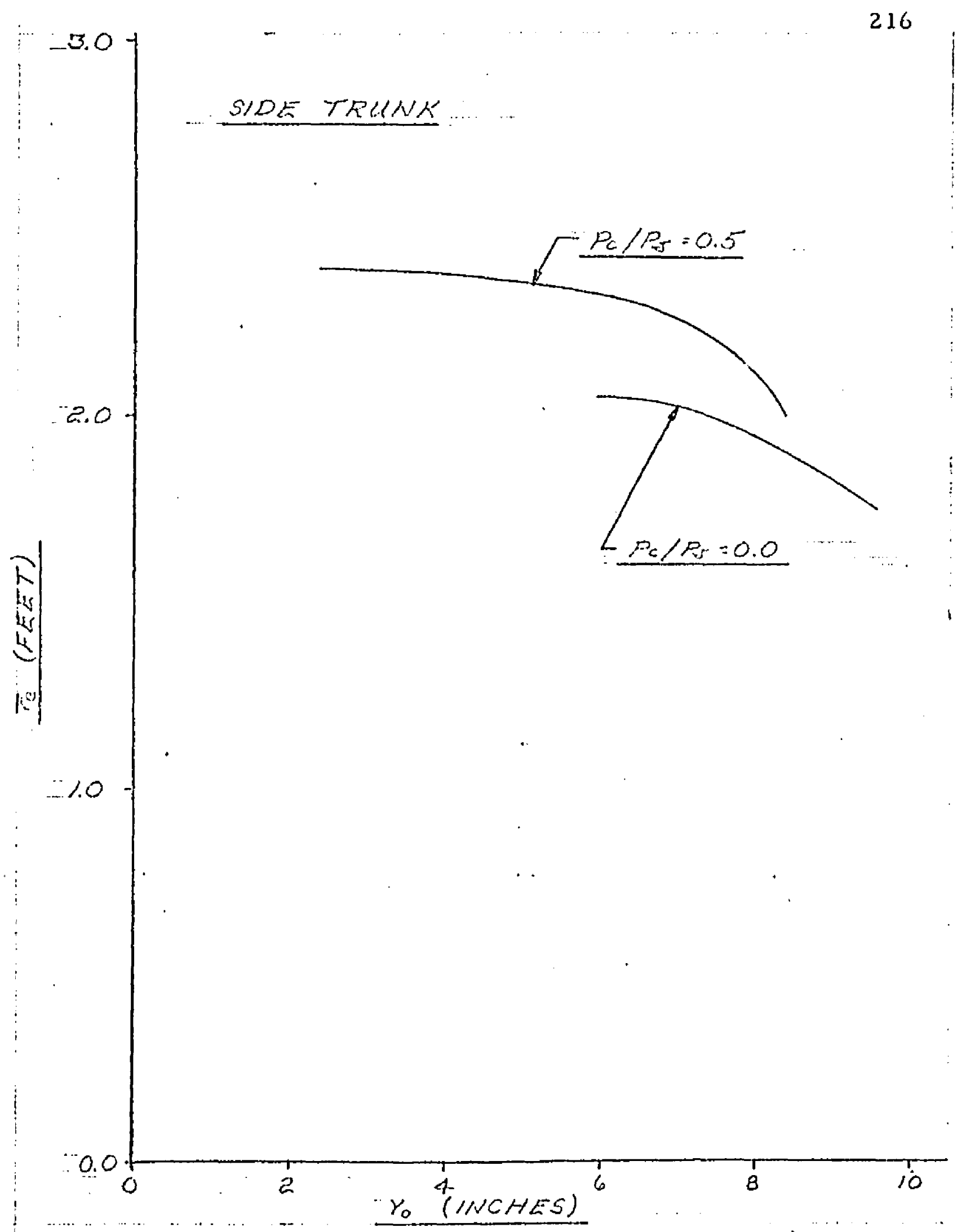

CENTROIDAL RADIUS VS TRUNK HEIGHT FOR MODEL TRUNK FIGURE 7-9 


\subsubsection{Influence of Cushion Pressure on Trunk Volume}

The influence of $\mathrm{P}_{\mathrm{c}} / \mathrm{p}_{\mathrm{j}}$ on trunk cross-sectional area for a side trunk section with free edges is shown in Figure 4-23. For the $p_{c}=0$ case, the relationship between $A_{j}$ and $Y_{0}$ is given by the $p_{c} / p_{j}=0$ curve. Higher values of $p_{c} / p_{j}$ tend to decrease $\ell_{3}$ for a given $Y_{0}$.

The total trunk volume is the product of the trunk cross-sectional area $\left(A_{j}\right)$ and the effective trunk length $\left(S_{j}\right)$.

The effective length for the two sides is $2 L_{5}$. The effective length for the two ends is the product of the distance from the center of revolution to the area centroid and the angle of revolution. The resulting equation for the trunk volume is:

$$
V_{j}=\left(2 L_{s}+2 \pi \overline{r_{e}}\right) A_{j}
$$

In Equation (7-63), $L_{s}$ is constant. The centroidal distance $\vec{r}_{e}$ and the cross-sectional area $A_{j}$ are dependent on both $p_{c} / p_{j}$ and $Y_{0}$. The dependence of these variables has been shown in Figures 7.9 and $4-23$, respectively.

\subsubsection{Influence of Cushion Pressure on Thrust}

The presence of cushion pressure reduces the exhaust velocity from the rows of orifices in the $\ell_{2}$ segment of the trunk. The effect of this reduction may be approxinated by adjusting Equation (7-26) to account for the cushion pressure across the $\ell_{2}$ segmerit. The resulting equation is:

$$
F_{j}=\left[\left(P_{j}-P_{c}\right) a_{2}+\left(P_{j}-P_{a}\right)\left(a_{j}-a_{2}\right) C_{y}\right] C_{x} C_{z}
$$

\subsubsection{Summary of Equations}

The changes required to apply the system of equations developed in Section 7.3 to the complete air cushion system have been developed in this section. The resulting 
equations may be summarized as follows:

\section{Definition of velocity}

$\frac{d y}{d t}=\dot{y}$

Newton's second law

$\frac{d \dot{y}}{d t}=\frac{g_{0}}{W_{A}}\left[-W_{A} \frac{g}{g_{0}}+A_{3}\left(P_{j}-P_{a}\right)+A_{c}\left(P_{c}-P_{a}\right)+F_{j}\right]$

First law of thermodynamics

$\frac{d P_{j}}{d t}=P_{j}\left(\frac{k}{W_{j}} \cdot \frac{d W_{j}}{d t}-\frac{k}{V_{j}} \frac{d V_{j}}{d t}\right)$

$\frac{d P_{c}}{d t}=P_{c}\left(\frac{k}{W_{c}} \frac{d W_{c}}{d t}-\frac{k}{V_{c}} \frac{d V_{c}}{d t}\right)$

Conservation of mass law

$\frac{d W_{i}}{d t}=\rho Q_{i}-\left[w_{1}+w_{2}+w_{3}\right]$
$\frac{d w_{c}}{d t}=w_{2}-\sqrt{2 g_{0} \frac{W_{c}}{V_{c}}\left(P_{c}-P_{a}\right)} s_{3} d C_{D}$

Geometric compatibility

$V_{j}=\left(2 L_{s}+2 \pi \bar{r}_{e}\right) A_{j}$ 


$$
V_{c}=\left\{A_{h}+X_{o}\left[L_{s}+\pi\left(\frac{e}{2}+\frac{X_{o}}{3}\right)\right]\right\} Y_{o}
$$

In the system of equations there are eight dependent variables: $y, \dot{V}, P_{j}, P_{C}, W_{j}$, $W_{c^{\prime}} V_{j}$, and $V_{c}$. The following variables are known and constant: $W_{A}, g_{O^{\prime}}, P_{a}, k, S_{3}, C_{D}, L_{s^{\prime}}$ $A_{h}, X_{0}, e, C_{X}$, and $C_{z}$.

The following variables are known functions of the dependent variables:

$$
\begin{aligned}
& F_{j}=\left(P_{j}-P_{c}\right) a_{2}+\left(P_{j}-P_{a}\right)\left(a_{j}-a_{2}\right) C_{y} \quad C_{x} C_{z} \\
& Y_{0}=-y \text { for } y<0 \quad \text { Assumption 7.3.1.7 } \\
& A_{c}=A_{h}+S_{g} X_{0} \\
& A_{3}=L_{s}+\pi\left[\left(\frac{e}{2}+x_{0}+l_{3}\right)^{2}-\left(\frac{e}{2}+x_{0}\right)^{2}\right] \\
& w_{1}=\sqrt{2 g_{0} w_{j} / V_{j}\left(P_{j}-P_{a}\right)} \quad a_{1} C_{x} \\
& w_{2}=(\operatorname{sign}) \sqrt{2 g_{0} \frac{w_{j}}{v_{j}}\left(P_{j}-P_{c}\right)} \quad a_{2} C_{x}
\end{aligned}
$$

where the sign takes the same sense as the quantity $\left(P_{j}-P_{c}\right)$.

$$
w_{3}=\sqrt{2 g_{0} \frac{w_{j}}{v_{j}}\left(P_{j}-P_{a}\right)} a_{3^{\prime}}
$$




$$
\begin{array}{ll}
a_{i} & =f\left(P_{j}\right) \text { as developed in Section 7.4.7. } \\
c_{y} & =f\left(Y_{0}\right) \text { as developed in Section 7.3.5. } \\
a_{1} & =f\left(Y_{0}, P_{c} / p_{j}\right) \text { as developed in Section 7.4.7. } \\
a_{2} & =f\left(Y_{0}, P_{c} / p_{j}\right) \text { as developed in Section 7.4.7. } \\
a_{3} & =f\left(Y_{0}, P_{c} / P_{j}\right) \text { as developed in Section 7.4.7. } \\
d & =f\left(Y_{0}\right) \text { as devoloped in Section 7.4.7. } \\
\overline{r_{e}} & =f\left(Y_{o}, P_{c} / P_{j}\right) \text { as developed in Section 7.4.9. }
\end{array}
$$

: A sufficient amount of information has been developed in this section to allow the prediction of the dynamic response of the complete air cushion landing system. Such a solution would require development of the functional relationships described above for a particular model. These relationships can be developed from analytical predictions by a procedure similar to that described in Chapter 8 . 


\section{EXPERIMENTAL PROGRAM - DYNAMIC MODEL}

\subsection{Experimental Apparatus - Dynamic Tests}

Figure 8-1 shows the test apparatus used for verification of the dynamic model developed in Chapter 7. The apparatus consisted of three units - a hydraulic power supply, a dynamic model, and a test platform.

Hydraulic power was supplied by a Sun Electric MK-3 Aircraft Hydraulic System Test Stand capable of delivering 0 to $30 \mathrm{gpm}$ at variable pressures up to $5,000 \mathrm{psig}$. The hydraulic power delivered to the dynamic model was regulated by controlling the flow rate pressure of the hydraulic fluid which was piped by flexible hoses to the model.

A drawing of the dynamic model is shown in Figure 4.8 and its dimensions are summarized in Table 8-I. The air source for the model was a centrifugal fan powered by a hydraulic motor. The fan and motor were connected by v-belts. The fan speed was 3.1 times the motor speed. The motor characteristics are shown in Figure 8-2. The fan characteristics are shown in Figure 8-3. Air was clucted from the fan into the trunk and exhausted from the trunk through 1093 holes located in the vicinity of the ground plane. The model structure was fiberglass and the trunk was a nylon-hypalon material. The trunk material was "inelastic" in that it did not possess the $200 \%$ to $300 \%$ elongation which would be required for complate retraction of the trunk. The elastic curve shown in Figure IV-3, Appendix IV, is typical for the trunk material.

The test platform was constructed of wood and was 10 feet in length by 8 feet in width. One section of the plywood surface was replaced with plexiglas in order to allow inspection of the underside of the test model. The center of the platform contained a 2 ' by $3^{\prime}$ hole which could be covered with plywood and sealed. The hole in the center allowed the cushion pressure to escape and, consequently, the performance of the trunk could be 

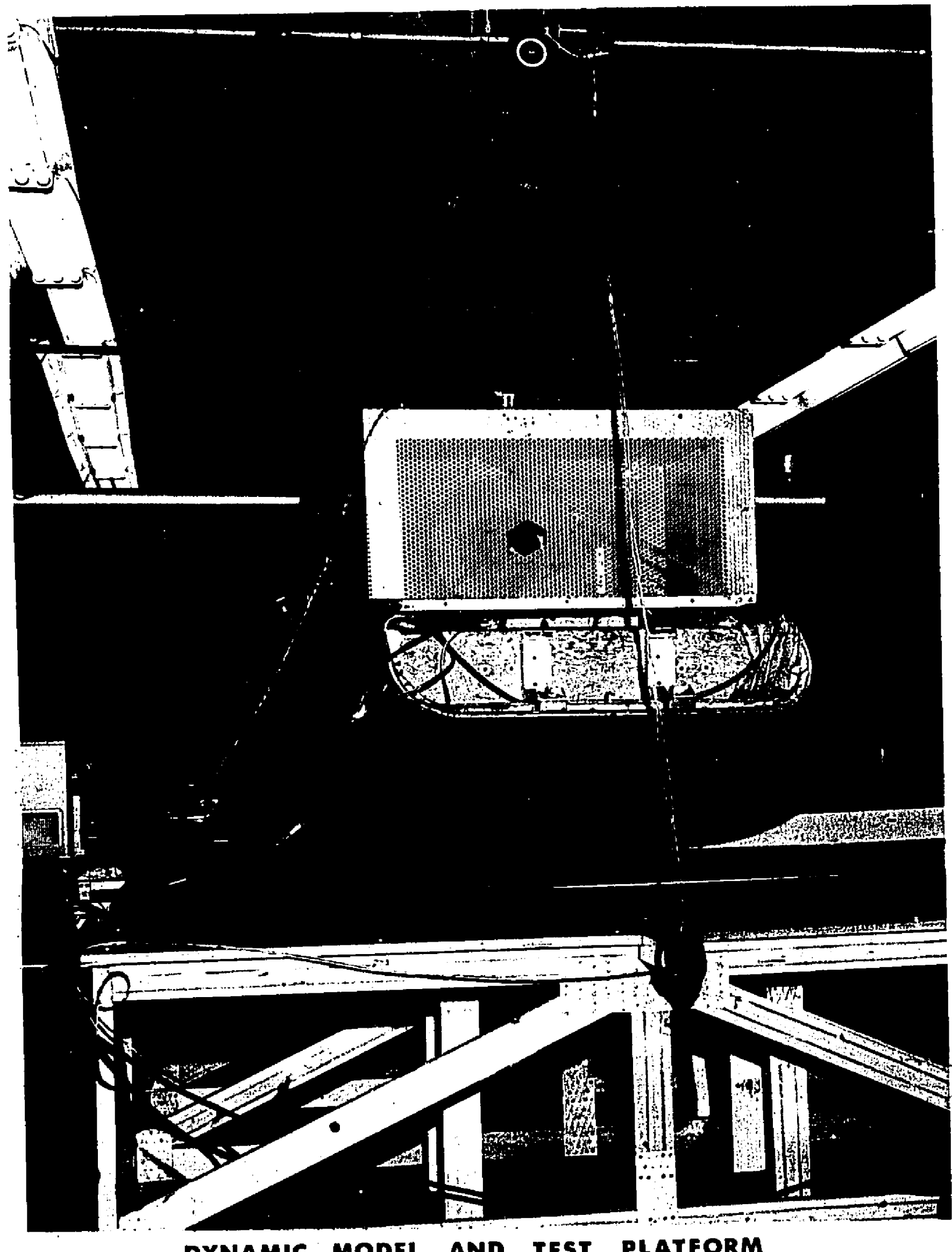
TABLE 8-1

Dynamic Model Trunk Design Variables

\begin{tabular}{|l|l|l|}
\hline \multicolumn{1}{|c|}{ VARIABLE } & SYMBOL & VALUE \\
\hline Total orifice area & $\mathrm{a}_{\mathrm{j}}$ & $104.16 \mathrm{in}^{2}$ \\
Number of orifices & $\mathrm{M}$ & 1093 \\
Porosity & $\xi$ & 0.049 \\
Cushion nozzle length & $\left(\mathrm{S}_{3}\right)_{\infty}$ & $14.7 \mathrm{ft}$. \\
\hline
\end{tabular}

Trunk Section Properties

\begin{tabular}{|l|c|c|c|c|}
\hline \multicolumn{1}{|c|}{ VARIABLE } & SYMBOL & $\begin{array}{c}\text { SIDE } \\
\text { SEC TION }\end{array}$ & $\begin{array}{c}\text { CORNER } \\
\text { SECTION }\end{array}$ & $\begin{array}{c}\text { END } \\
\text { SEC TION }\end{array}$ \\
\hline Cross-sectional area, in & $\left(\mathrm{A}_{\mathrm{j}}\right)_{0}$ & 326.1 & 235.6 & 202 \\
Effective section length, in & $\mathrm{L}_{\mathrm{N}}$ & 16.0 & 17.1 & 14.6 \\
$\begin{array}{l}\text { Section angle of rotation, } \\
\text { degrees }\end{array}$ & $\alpha_{\mathrm{n}}$ & 0 & 48 & 42 \\
Centroidal radius, in & $\gamma_{\mathrm{n}}$ & 20.9 & 20.4 & 19.9 \\
\hline
\end{tabular}


PERFORMANEE CURVES

FOR CONSTANT DISPLACEMENT MOTOR MODEL MF-3918-30 SERIES

\section{CONSTANTS}

OPERATING PRESSURE_- 3000 P SI. SPEED $---D----3600$ RPM. STROKE ANGLE_... OIL_-_-_-_- $-M I L-0-3606$ TENAPERATURE__._._- $150^{\circ} \pm 5^{\circ} \mathrm{F}$.

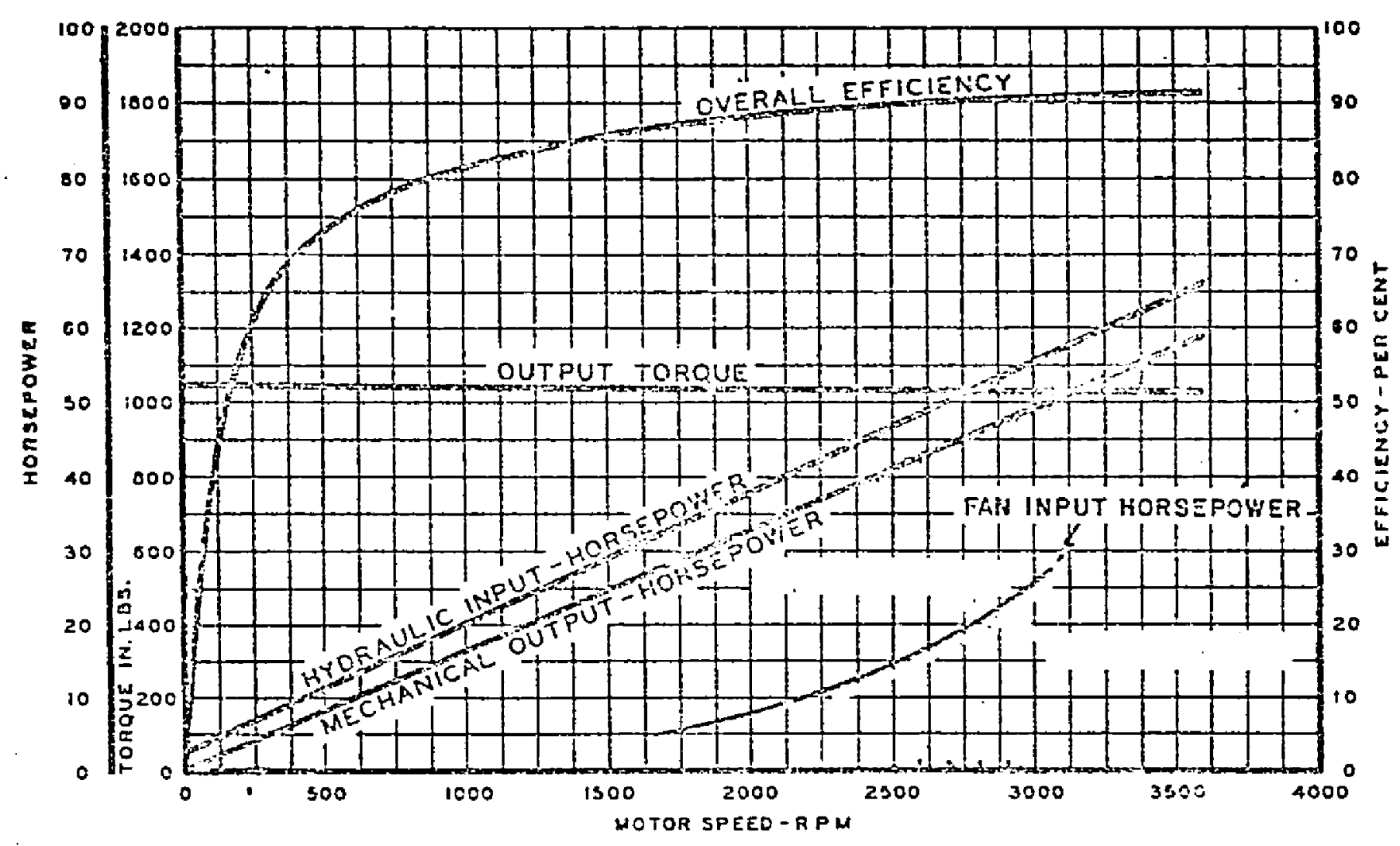

HYDRAULIC MOTOR CHARACTERISTICS 


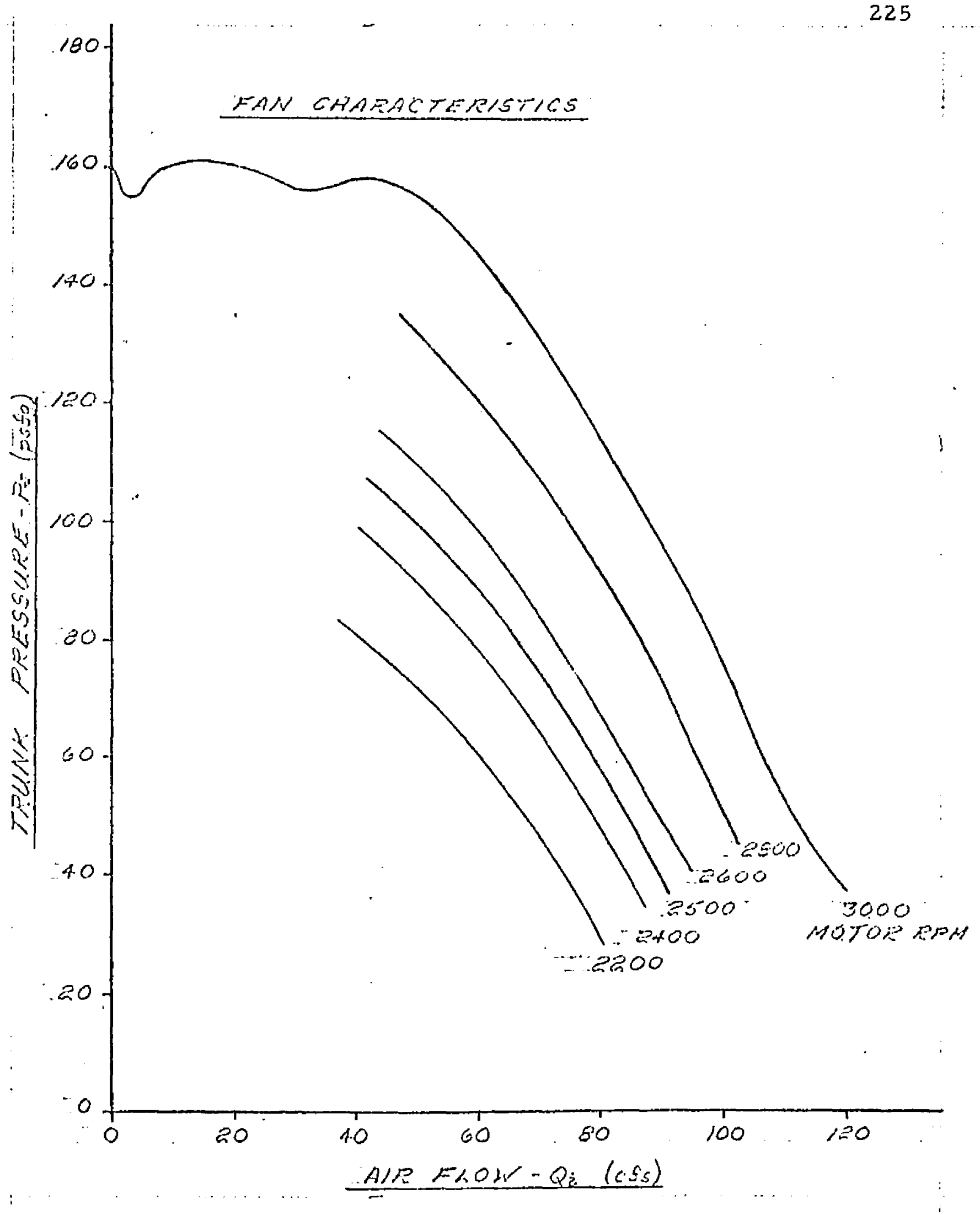

FAN CHARACTERISTICS

FIGURE 8-3 
measured independently from the cushion. The performance of the combined trunk-cushion system could be measured when the hole was closed and sealed.

Two types of tests were conducted. The first was a series of static tests to determine the static performance of the model and to compare the results with the analysis presented in Sections 7.3.2 through 7.3.6. During these tests the values of the following variables were determined: $C_{x}, F_{j}, C_{z}, A_{3}, C_{y}, d, A_{j}$, and $Q_{j}$. The results of these tests are reported in Sections 8.2 through 8.6.

The second test was a dynamic drop test of the model to determine the dynamic response and compare the results with the analysis presented in Section 7.3.7. In all tests reported, the value of $n_{c}$ was zero. The dynamic test is described in Section 8.7.

The variables used in this chapter are summarized in Chapter 7.

8.2 Determination of Discharge Coefficient $\mathrm{C}_{\mathrm{x}}$

A test was conducted to determine the discharge coefficient for the orifices in the trunk of the dynamic model. This test was conducted with the model suspended two feet above the test platform. At this distance, no cushion pressure existed and the influence of the ground plane on flow from the trunk was negligible.

By varying the hydraulic flow rate to the motor, the fan speed was varied to produce a trunk pressure which ranged from 25 to $65 \mathrm{psfg}$. For each data point, the rjom of the fan $(N)$ was determined with a strobe light and the trunk pressure $\left(p_{j}\right)$ was determined by a water filled manometer. The total air flow from the trunk was determined by entering $P_{j}$ and $N$ in Figure $8-3$ and reading $Q_{j}$. The coefficient of discharge was determined from Equation (VI-2), Appendix VI.

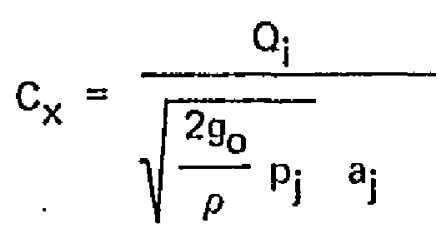

(see Appendix VI) (VI-2) 


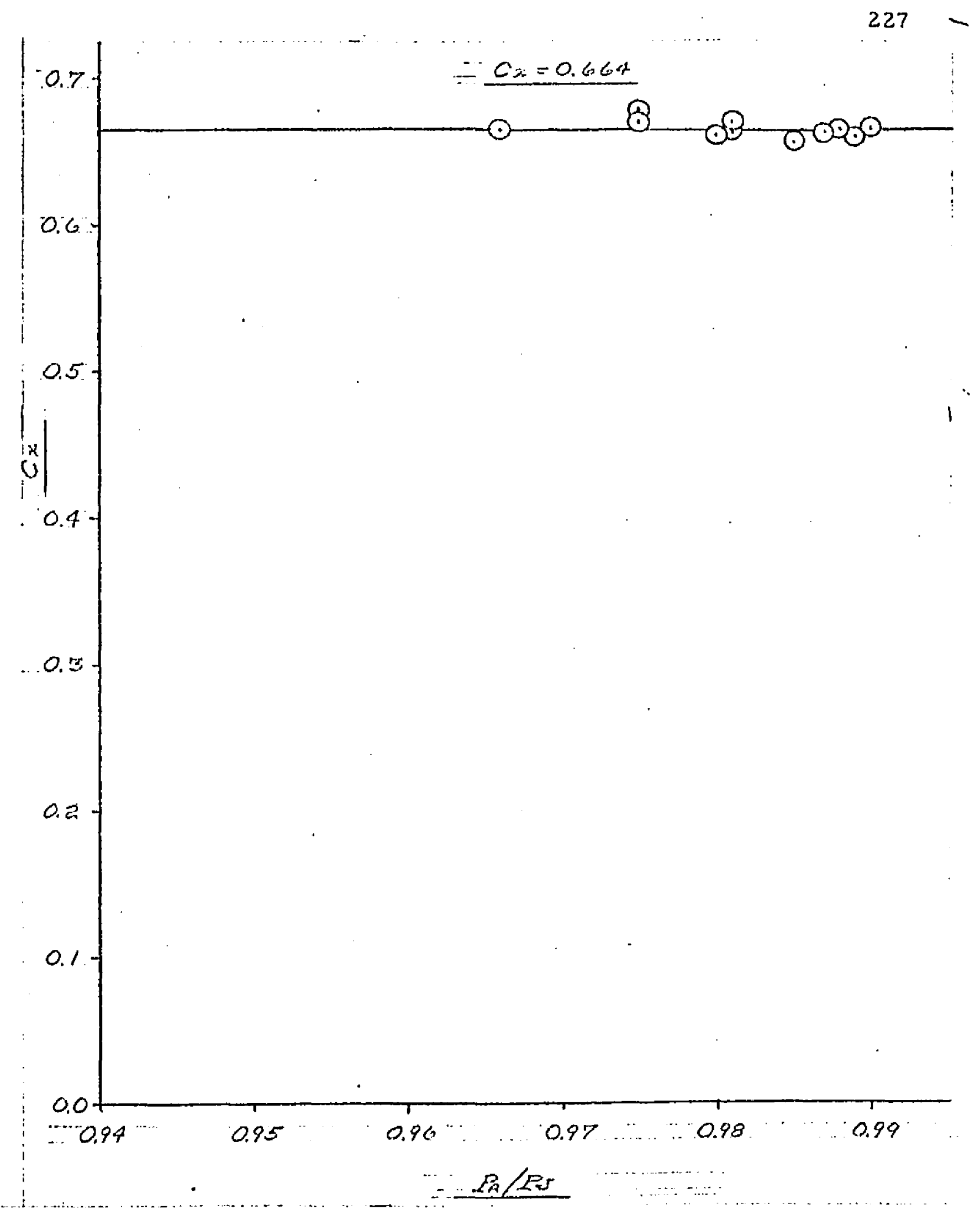

TRUNK DISCHARGE COEFFICIENT VS $P_{A} / P_{J}$ FIGURE $8-4$. 


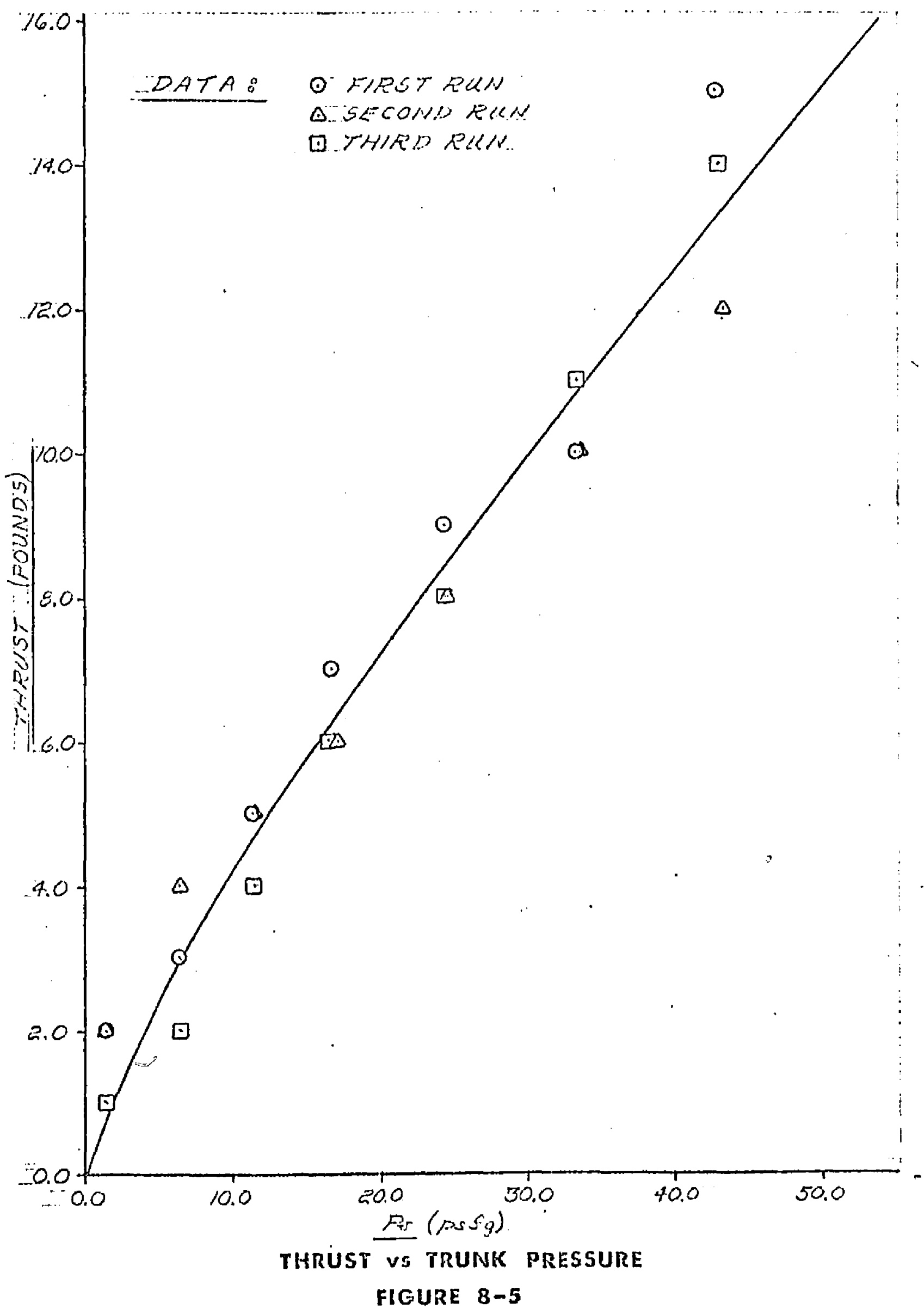


The resulting graph of $\mathrm{p}_{c} / \mathrm{p}_{j}$ versus $\mathrm{C}_{x}$ is shown in Figure 8-4. From Figure 8-4 it is evident that $C_{x}=0.66$ for the pressure range investigated.

\section{3}

$$
\text { Determination of Jet Thrust and } C_{z}
$$

In the test to measure vertical jet thrust, the model was suspended from a load cell. The model height was in excess of two feet so that the influence of the ground plane was negligible. The trunk pressure was varied from 0 to $45 \mathrm{psfg}$ and the loss of weight registered by the load cell was recorded. The vertical thrust was equated to the difference between the static weight and the weight recorded at a given trunk pressure. The resulting thrust versus $p_{j}$ was plotted in Figure 8.5.

The thrust coefficient $\left(C_{z}\right)$ was calculated from Equation (7-26).

$$
C_{z}=\frac{F_{j}}{2\left(P_{j}-P_{a}\right) a_{j} C_{x} C_{y}}
$$

For the test conducted:

$\mathrm{C}_{\mathrm{X}}=0.66$ (from Section 8.2)

$c_{y}=1.00($ from Section 7.3.5, Equation (7.39) )

The resulting value of $\mathrm{C}_{z}$ was found to be

$$
C_{z}=0.33
$$

8.4 Determination of $A_{3}$ and $C_{y}$

The variation of $A_{3}$ and $C_{y}$ with model height was determined from a test series which statically loaded the model against the test platform. The center section of the test platform was uncovered so that no cushion pressure existed. 


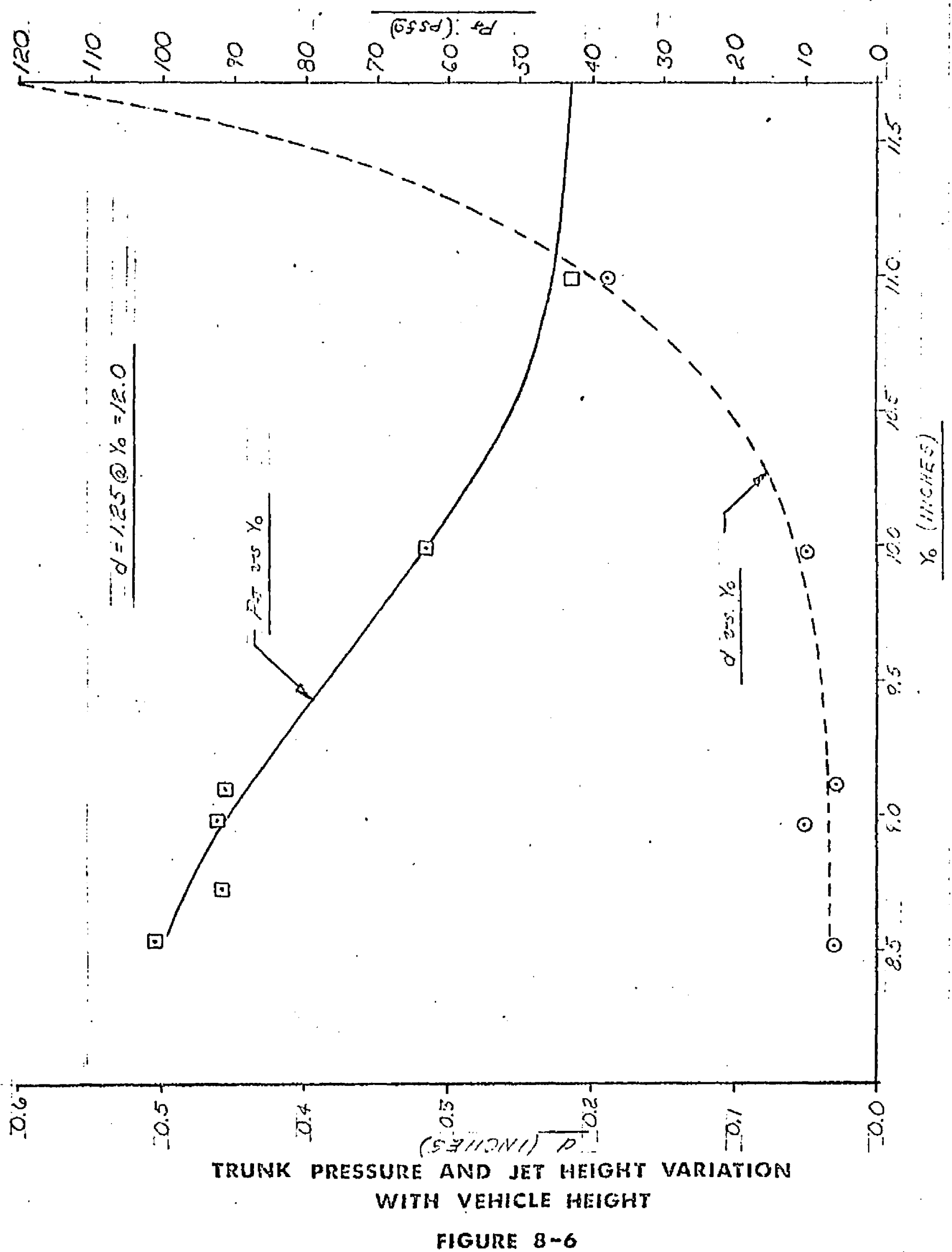




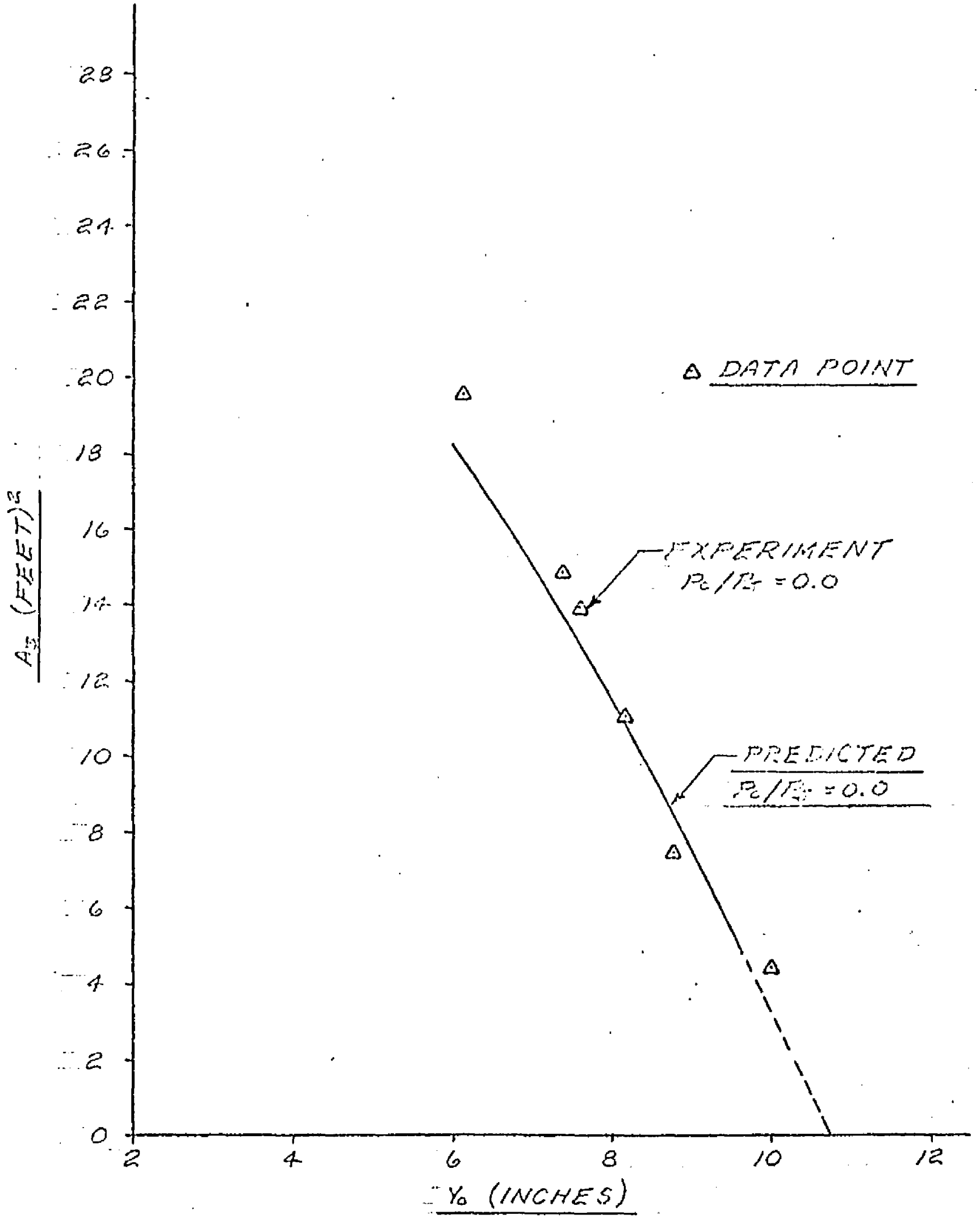

FOOTPRINT AREA VS VEHICLE HEIGHT

FIGURE $8-7$ 
The fan speed was maintained at a constant rpm and the weight supported by the trunk was varied. For low trunk loads, the model was partially suspended from a load cell. The load on the trunk was determined by the loss of weight registered by the load cell. For heavier loads, the model was loaded with known quantities of lead weights.

Data was recorded at approximately 1 -inch increments over a model height range from 11 inches to 7 inches. At each data point, the model was leveled by adjusting the location of weights and the fan speed was set at $8000 \mathrm{rpm}$. The trunk height $\left(Y_{0}\right)$ was measured with a scale and the jet height (d) was measured by rods of calibrated thickness. The trunk jressure was measured by a water tube manometer.

The recorded values of jet height $(d)$ and trunk pressure $\left\langle p_{j}\right\rangle$ at a constant fan speed of $8000 \mathrm{rpm}$ are shown in Figure 8-6.

The effective footprint areas of the trunk were calculated from the weight supported and the trunk pressure.

$$
A_{3}=\frac{W_{A}}{p_{j}}
$$

The resulting experimentally determined values of $A_{3}$ versus $Y_{0}$ are shown in Figure 8.7.

The values of $A_{3}$ calculated by the computer program cleveloped in Section 4.5 were also plotted in Figure 8-7. Values of $A_{3}$ were computed from the values of $l_{3}$ shown in Figures 4-21 and 4.22 using techniques described in Sections 7.3.3 and 7.4.8. In computing $A_{3}$, the trunk was divided into three parts - the ends, the sides, and the corners. These three parts are designated by $L_{e}, L_{s}$, and $L_{k}$ respectively (see Figure 4-8). The respective footprint areas were computed as follows.

$$
\begin{aligned}
& \left(A_{3}\right)_{s}=\left(\ell_{3}\right)_{s} L_{s} \\
& \left(A_{3}\right)_{e}=\frac{(a)_{e}}{2}\left\{\left[\frac{e}{2}+\left(X_{0}\right)_{e}+\left(\ell_{3}\right)_{e}\right]^{2}-\left[\frac{e}{2}+\left(X_{o}\right)_{e}\right]^{2}\right\}
\end{aligned}
$$




$$
\begin{aligned}
\left(A_{3}\right)_{k}= & \frac{(a)_{k}}{4}\left\{\left[\frac{\mathrm{e}}{2}+\left(x_{0}\right)_{\mathrm{e}}+\left(l_{3}\right)_{\mathrm{e}}\right]-\left[\frac{\mathrm{e}}{2}+\left(x_{\mathrm{o}}\right)_{\mathrm{e}}\right]^{2}\right. \\
& \left.+\left[\frac{\mathrm{e}}{2}+\left(\mathrm{x}_{\mathrm{o}}\right)_{\mathrm{s}}+\left(l_{3}\right)_{\mathrm{s}}\right]^{2}-\left[\frac{\mathrm{e}}{2}+\left(\mathrm{x}_{\mathrm{o}}\right)_{\mathrm{s}}\right]^{2}\right\}
\end{aligned}
$$

As shown in Equation (7.28), the total footprint area is equal to the sum of the various parts. For the model in Figure 4-8

$$
A_{3}=2\left(A_{3}\right)_{e}+2\left(A_{3}\right)_{s}+4\left(A_{3}\right)_{k}
$$

Figure 8.7 shows good agreement between the footprint area predicted by Equation (8.6) and the area determined by experiment.

The value of $\mathrm{C}_{y}$ was also computed from theoretical considerations. For this calculation, the trunk model was divided into three segments: $\ell_{1}, \ell_{2}$, and $\ell_{3}$ as shown in Figure 4-2. The flow from the three segments was computed following the procedure outlined in Section 7.3.5. The resulting equations were:

$$
c_{y}=\frac{a_{j}+A_{3} \xi-a_{3}^{\prime}}{a_{j}}
$$

and

$$
a_{3}^{\prime}=\left\{\begin{array}{l}
A_{3} \xi \\
2 s_{3} d C_{D} / C_{X}
\end{array}\right\} \quad \text { whichever is less }
$$

The values of $a_{j}, \xi$, and $S_{3}$ are given in Table 8-I. The values of $C_{X}$ and $C_{D}$ were experimentally determined in Appendix VI and Section 6.3.2 respectively. The values for $d$ 
234

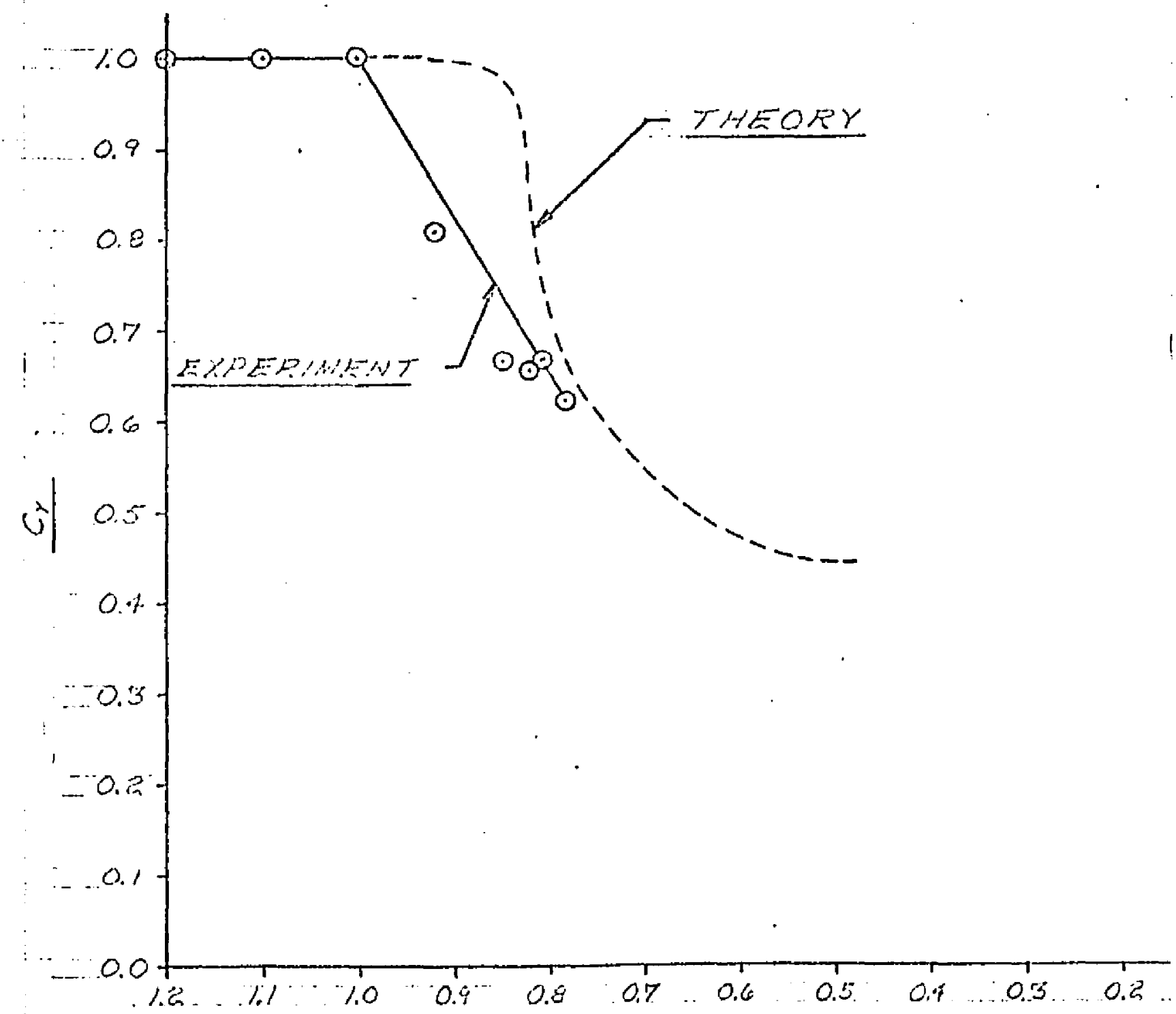

$$
\ldots . . . y_{0} / \gamma_{0} \quad\left(Y_{00}=10.511 / \mathrm{CHES}\right)
$$

CUSHION DISCHARGE COEFFICIENT VS VEHICLE HEIGHT

FIGURE 8-8 
and $A_{3}$ versus $Y_{0}$ are given in Figures 8-6 and 8-7 respectively. The resulting variation of $c_{\gamma}$ with $Y_{0}$ is given in Figure 8-8.

\subsection{Determination of Trunk Volume}

A test was conducted to determine the variation in trunk volume with model height.

The trunk volume in the free (unloaded) condition was determined by graphical integration of the various cross-sectional areas shown in Figure 4.8. The total trunk volume was found to be $25.24 \mathrm{ft}^{3}$. The volume of the ducting between the fan and trunk was 1.8 $\mathrm{ft}^{3}$.

The change in volume with model height was determined from the change in trunk cross-sectional area as the model was statically loaded against the test platform.

A constant fan speed of $8,000 \mathrm{rpm}$ was used for this test. The floor center sections were removed to prevent cushion pressure build-up and to allow access to the inside portion of the trunk. The model height was varied by changing the load which was supported by the trunk. Data points were taken at approximately every 1.5 inches from a model height of 12 inches down to 6.25 inches. The trunk shape was determined at the midpoint of one side and one end for each data point.

The ground tangent points, $\left(x_{1}, y_{0}\right)$ and $\left(x_{2}, y_{0}\right)$ in Figure 4-2, were determined by measuring the vertical and horizontal distance relative to the attachment points $(0,0)$ and $(a, b)$.

The contour between an attachment point and a ground tangent point was determined by fitting a copper wire against the trunk. The copper wire was deformed plastically to retain the trunk contour. The inside and outside contours $l_{2}$ and $\ell_{1}$ in Figure 4-2, respectively) were transferred by the copper wire to a full scale drawing of the trunk cross section. The resulting areas were measured with a compensating polar planimeter. 
The volume of the trunk was calculated from the side and end cross-sectional areas in a manner similar to that outlined in Section 7.3.4. In order to perform this calculation, the trunk volume was separated into the four parts - the end shape, the corner shape, the side shape, and the fan ducting. These parts are shown in Figure 4-8. It is evident from the figure that the total volume of the trunk is

$$
v_{j}=2 v_{e}+2 v_{s}+4 v_{k}+v_{f}
$$

The volume of the end is a volume of revolution. The radius vector between the center of revolution and the centroid of the cross-sectional area is $\vec{r}_{\mathrm{e}}$. The total volume of the two end sections is the product of the angle of revolution, the radius and the cross-sectional area. The result is:

$$
v_{e}=a_{e} \overline{r_{c}}\left(A_{j}\right)_{c}
$$

The volume of the two sides is the product of the section length $L_{S}$ and the cross-sectional area. The result is:

$$
v_{s}=L_{s}\left(A_{j}\right)_{s}
$$

The volume of a corner section is more difficult to calculate than the other volumes. It approaches a volume of rotation, however the cross-sectional area and the radius of the centroid vary with the angle of rotation. On one side the cross-sectional area is $\left(A_{j}\right)_{e}$ and the centroid radius is $\bar{r}_{e}$. On the other side the cross-sectional area is $\left(A_{j}\right\rangle_{s}$ and the centroidal radius is $\overrightarrow{r_{s}}$. It is evident that the volume of a single corner section lies in the range

$$
a_{e} \bar{r}_{e}\left(A_{j}\right)_{e}<V_{k}<a_{s} \bar{r}_{s}\left(A_{j}\right)_{s}
$$


In orcler to approximate the corner volume, the following assumptions were made.

(1) The effective centroidal radius for the corner section is the average of the end and side radii.

$$
\bar{r}_{k}=\frac{\bar{r}_{\mathrm{c}}+\overline{\mathrm{r}}_{\mathrm{s}}}{2}
$$

(2) The values of $\overline{r_{e}}$ and $\overline{r_{s}}$ do not change with $Y_{o}$.

(3) The effective cross-sectional area of the corner section lies somewhere between $\left(A_{j}\right)_{e}$ and $\left(A_{j}\right)_{s}$,

$$
\left\langle A_{j}\right\rangle_{k}=\left\langle A_{j}\right\rangle_{s}+(1-\zeta)\left(A_{j}\right\rangle_{e}
$$

where $\zeta$ is a fraction between 0.0 and 1.0 .

The resulting corner volume is:

$v_{k}=a_{k} \frac{\left(r_{e}+r_{s}\right)}{2}\left[\zeta\left(A_{j}\right)_{s}+(1-\zeta)\left(A_{j}\right)_{e}\right]$

The total trunk volume may now be written:

$$
\begin{aligned}
v_{j}= & 2\left[a_{e} \bar{r}_{e}\left(A_{j}\right)_{e}\right]+2\left[L_{s}\left(A_{j}\right)_{s}\right] \\
& +4\left\{a_{k} \frac{\left(\bar{r}_{e}-\bar{r}_{s}\right)}{2}\left[(1-\zeta)\left(A_{j}\right)_{e}+\zeta\left(A_{j}\right)_{s}\right]\right\}+v_{f}
\end{aligned}
$$


Factoring the above equation gives:

$$
\begin{aligned}
& v_{j}=\left[2 a_{\mathrm{e}} \bar{r}_{\mathrm{e}}+4 a_{\mathrm{k}} \frac{\left(\overline{\mathrm{r}}_{\mathrm{e}}+\overline{\mathrm{r}}_{\mathrm{s}}\right)}{2}(1-\zeta)\right] \quad\left(\mathrm{A}_{\mathrm{j}}\right\rangle_{\mathrm{e}} \\
& +\left[2\left(l_{\mathrm{s}}\right)+4 a_{k} \frac{\left\langle\bar{r}_{\mathrm{e}}+\bar{r}_{\mathrm{s}}\right\rangle}{2}(\zeta)\right]\left\langle\mathrm{A}_{\mathrm{j}}\right\rangle_{\mathrm{s}}+\mathrm{V}_{\mathrm{f}}
\end{aligned}
$$

The free volume of the trunk may be written

$$
\begin{aligned}
\left(V_{j}\right)_{\infty}= & {\left[2 a_{e} \bar{r}_{e}+4 a_{k} \frac{\left(\bar{r}_{c}+\bar{r}_{s}\right)}{2}(1-\zeta)\right]\left(A_{j}\right)_{\infty} } \\
+ & {\left[2\left(L_{s}\right)+4 a_{k} \frac{\left\langle\bar{r}_{e}+\bar{r}_{s}\right\rangle}{2}(\zeta)\right]\left(A_{j}\right)_{\infty}+V_{f} }
\end{aligned}
$$

With the exception of $\zeta$, the values of all variables in the above equation are known and are listed in Table 8-1. Consequently, the equation may be solved for $\xi$. For the model summarized in Table 8-1 the value of $\zeta$ was 0.727 .

It is now possible to simplify Equation (8-18) with the following condensation of variables: "

$$
\begin{aligned}
& K_{e} \equiv 2 a_{e} \bar{r}_{e}+4 a_{k} \frac{\left(\bar{r}_{e}+\bar{r}_{s}\right)}{2}(1-\zeta) \\
& K_{s} \equiv 2 L_{s}+4 a_{k} \frac{\left(\bar{r}_{e}+\bar{r}_{s}\right)}{2} \zeta
\end{aligned}
$$

For the model described in Table 8.1, the valucs of these parameters are $K_{e}=$ $126.6 \mathrm{in.}$ and $\mathrm{K}_{\mathrm{s}}=55.5 \mathrm{in}$. 


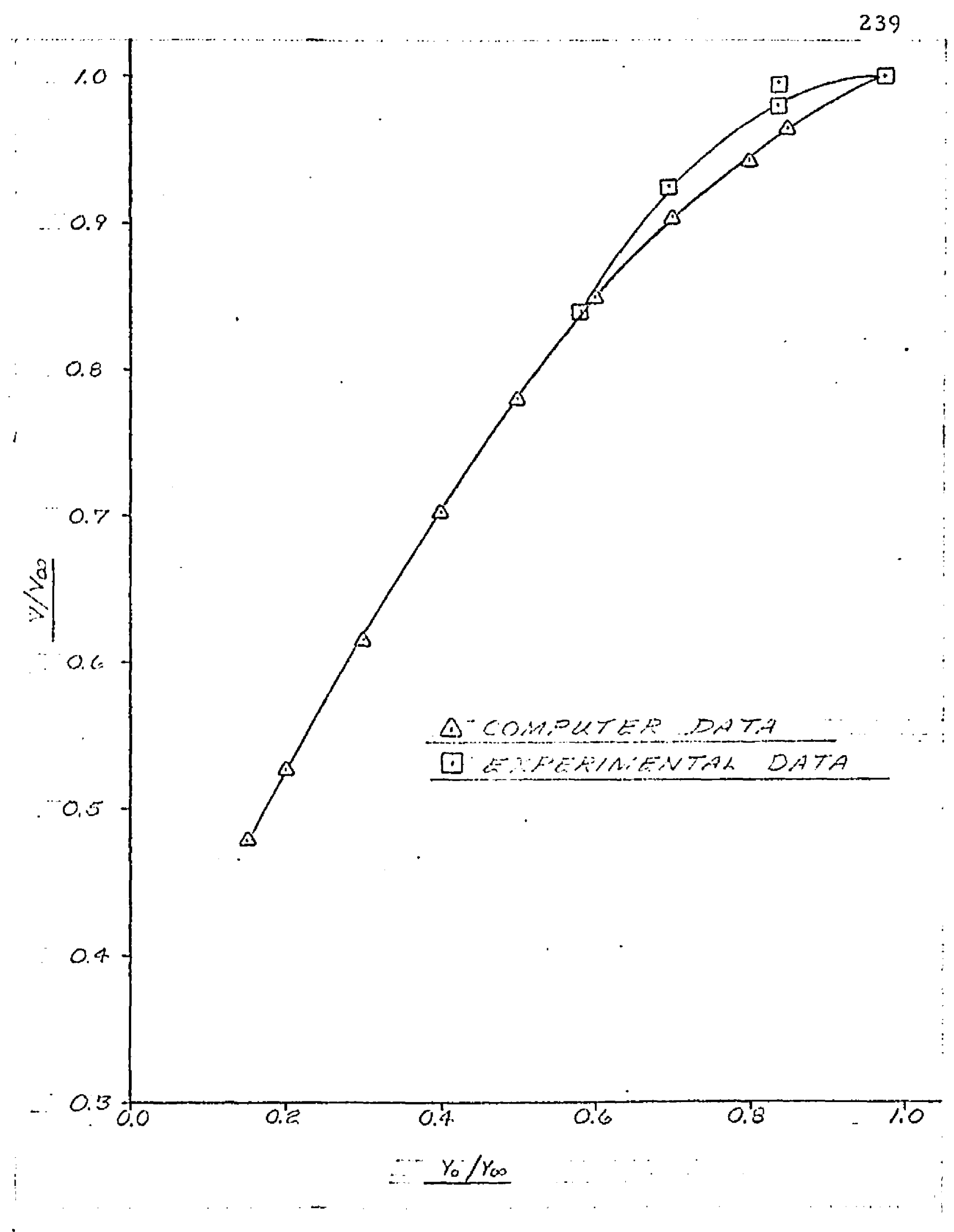

TRUNK VOLUME RATIO VS VEHICLE HEIGHT RATIO

FIGURE 8-9 
The general equation for the total volume of the trunk and ducting may be written

$$
v_{j}=K_{e}\left(A_{j}\right)_{e}+K_{s}\left(A_{j}\right)_{s}+V_{f}
$$

The volume ratio is

$$
\left\langle V_{j}\right)_{\infty}=\frac{K_{e}\left(A_{j}\right)_{\mathrm{e}}+K_{\mathrm{s}}\left(A_{j}\right)_{s}+V_{f}}{\left(V_{j}\right)_{\infty}}
$$

Figure 8-9 shows the values of $V_{j} /\left(V_{j}\right)_{\infty}$ computed from the experimentally determined values of $\left(A_{j}\right)_{e}$ and $\left(A_{j}\right\rangle_{s}$. In addition, the values of $V_{j} / V_{j \infty}$ computed from the values of $A_{j}$ predicted by the computer program developed in Chapter 4 are shown. The computed values of $\left(A_{j}\right)_{s}$ and $\left(A_{j}\right)_{e}$ versus $Y_{0} / Y_{\infty}$ are shown in Figures 4-23 and 4-24.

\subsection{Fan Characteristics}

The fan cluaracteristics were determined by measuring the flow from the fan at various speeds and back pressures.

In the calibration tests, the fan and ducting were installed above a plywood plenum chamber of approximately the same volume as the free volume of the trunk. Two convergent conical nozzles with an included angle of 12 degrees were installed on opposite sides of the plenum. The discharge coefficient of the conical nozzles was constant at 0.95 over the range of Reynolds numbers of interest in the test. Data was recorded at $200 \mathrm{rpm}$ increments, at motor speeds ranging between $2200 \mathrm{rpm}$ and $3000 \mathrm{rpm}$. The back pressure (trunk pressure) was varied by changing the exit area of the convergent nozzles. Since the coefficients for the nozzles were known, the total flow from the fan could be calculated from the formula given in Appendix VI. 
241

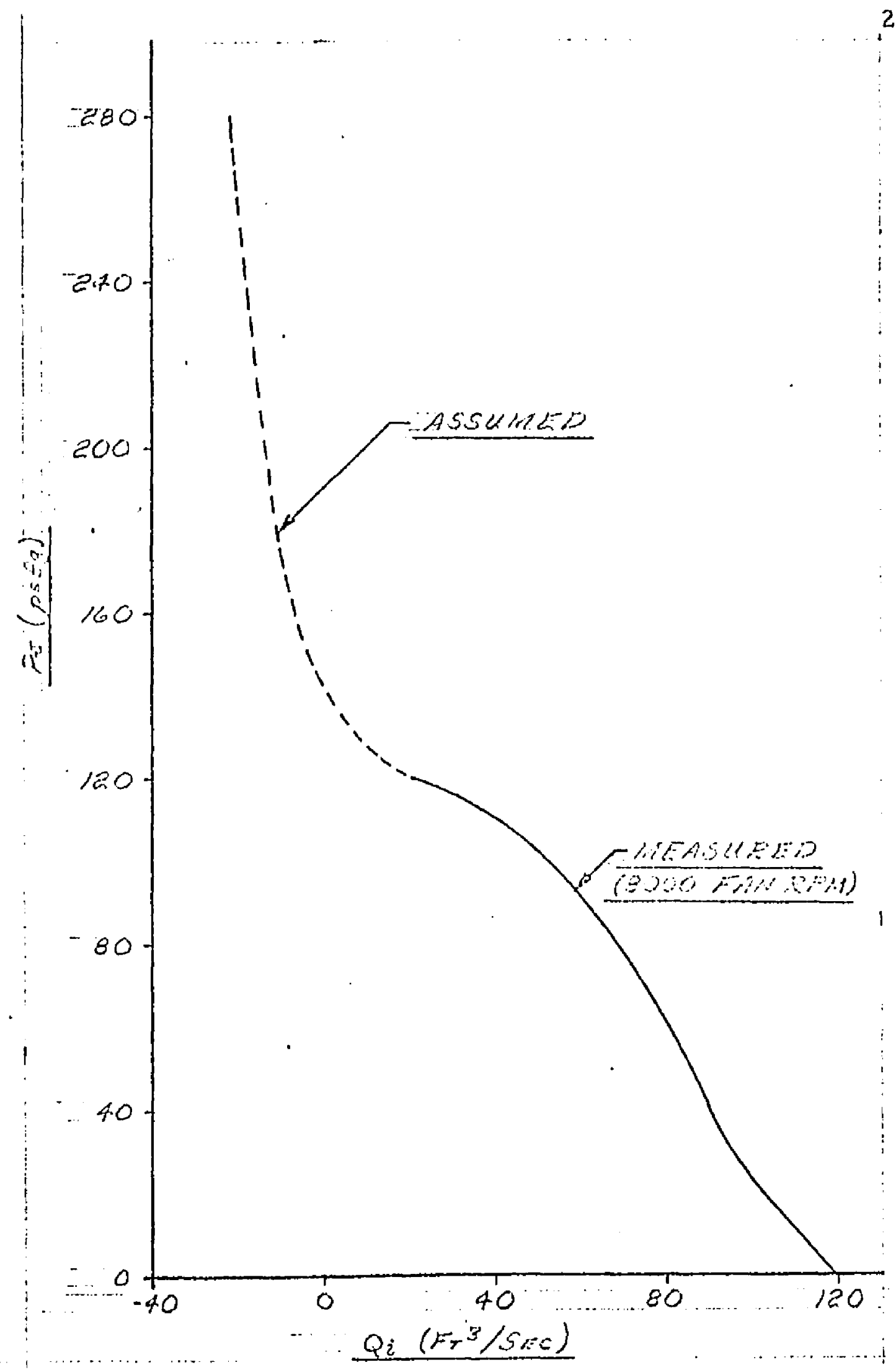

ASSUMED FAN CHARACTERISTICS

FIGURE $8-10$ 


$$
\mathrm{Q}_{\mathrm{j}}=\sqrt{\frac{2 \mathrm{~g}_{\mathrm{o}}}{\rho}\left(\mathrm{p}_{\mathrm{j}}\right)} \mathrm{a}_{\mathrm{D}} \mathrm{C}_{\mathrm{D}}
$$

where

$a_{D}$ is the total exhaust nozzle area

$C_{D}$ is the nozzle coefficient of discharge

The resulting fan characteristics of the fan were plotted in Figure 8-3.

The backflow characteristics of the fan were not known. Consequently, it was assumed that at pressures above the stall pressure of the fan, the backflow through the fan was proportional to the square root of the difference between trunk pressure and stall pressure. The resulting relationship was:

$$
a_{i}=a_{r}-\sqrt{\frac{2 g_{o}}{\rho}\left(P_{r}-P_{j}\right)} a_{r}
$$

where

$P_{r}$ is the stall pressure of the fan for $8000 \mathrm{rpm}$ fan speed

$\mathrm{O}_{r}$ is the flow at the stall pressure

$a_{r}$ is the effective flow area associated with fan backflow.

The assumed relationship between $\mathrm{O}_{\mathfrak{i}}$ and $\mathrm{P}_{\mathrm{j}}$ at pressures above stall pressures is shown in Figure 8-10.

\subsection{Dynanic Model Test}

In order to verify the dynamic analysis developed in Section 7-2, the dynamic test model was allowed to free fall and impact against the platform. Prior to drop test, the model was suspended above the platform by a nylon belt which incorporated a quick relcase 
mechanism. The height above the platform and the fan rpm were measured prior to drop by a scale and a strobe light, respectively. During the drop and subsequent impact, the following parameters were measured and recorded.

$p_{c}$ and $p_{j}$ were measured by Consolidated Electrodynamic Type 4-312 pressure transducers located in the cushion and trunk areas. These instruments had a pressure range of $t=12.5$ psi with a linearity of $\pm 1.0 \%$ of the full scale reading. The natural frequency of the instruments was 8,000 cps. The error caused by a $15 \mathrm{~g}$ peak sinusoidal vibration from 5 to $2,000 \mathrm{cps}$ was less than $\pm 0.160 \%$ full range $/ \mathrm{g}$.

(2) Vertical acceleration was measured by a model $333 \mathrm{~g}$ Stradham Laboratories accelerometer with at $25 \mathrm{~g}$ range, and a linearity of \pm $1.0 \%$ of full scale reading.

(3) The vertical displacement was measured by a linear displacement transducer, Model 4040 manufactured by Research, Incorporated. The displacement transducer had a $3.0 \mathrm{ft}$ range, with a linearity of $\pm 1.0 \%$ of full scale reading.

The data was recorded on a direct reading oscillograph, Data Graph Model 5-26 manufactured by Consolidated Electrodynamics Corporation. The paper speed was eighteen inches per second.

The recorded values of trunk pressure, vertical acceleration, and vertical displacement are shown in Figures 8-11, 8-12, and 8-13, respectively, for a typical drop test. For the test results shown, the drop height was one foot and the cushion pressure was zero. 


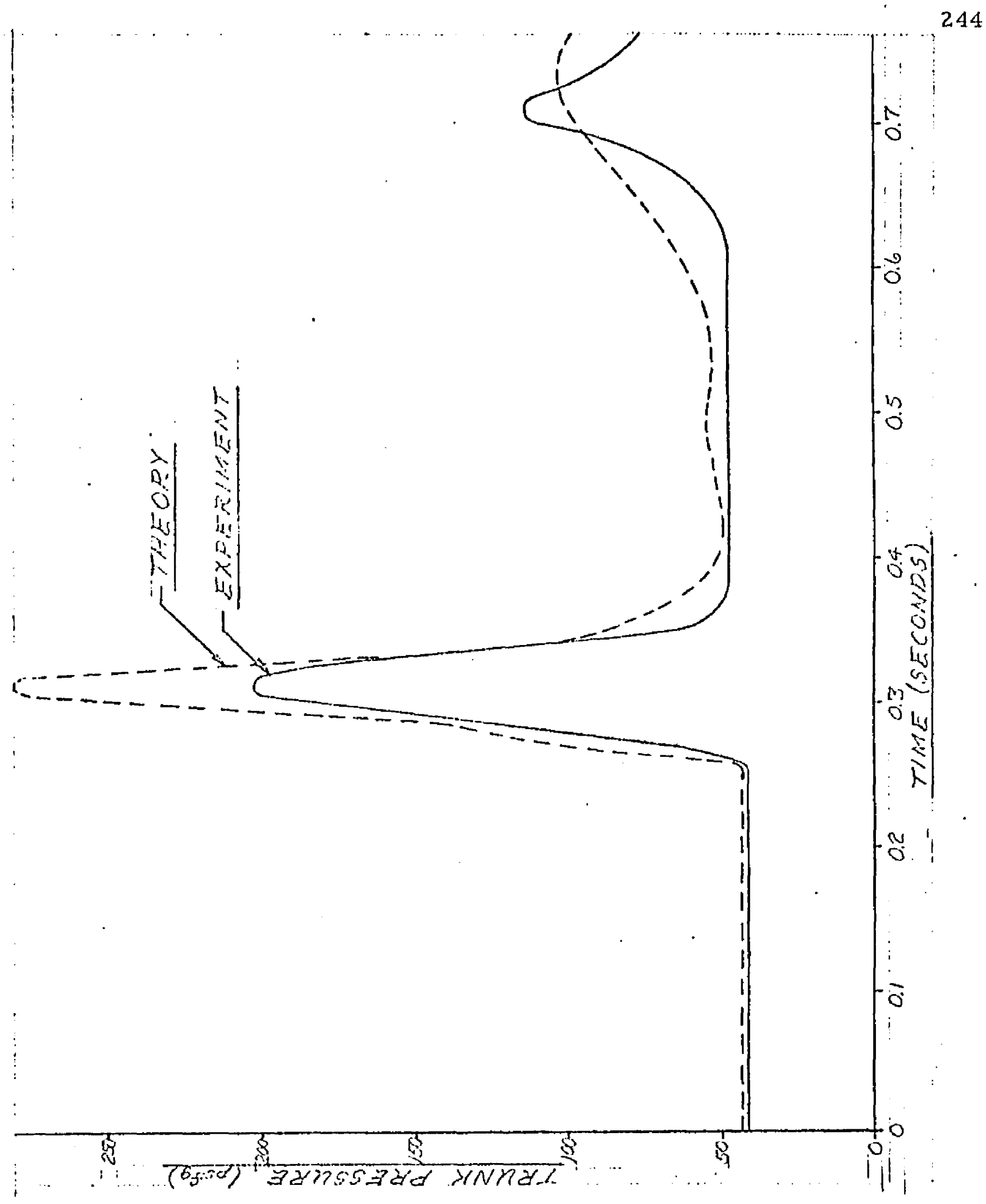

TRUNK PRESSURE DURING DROP TEST FIGURE $8-11$ 


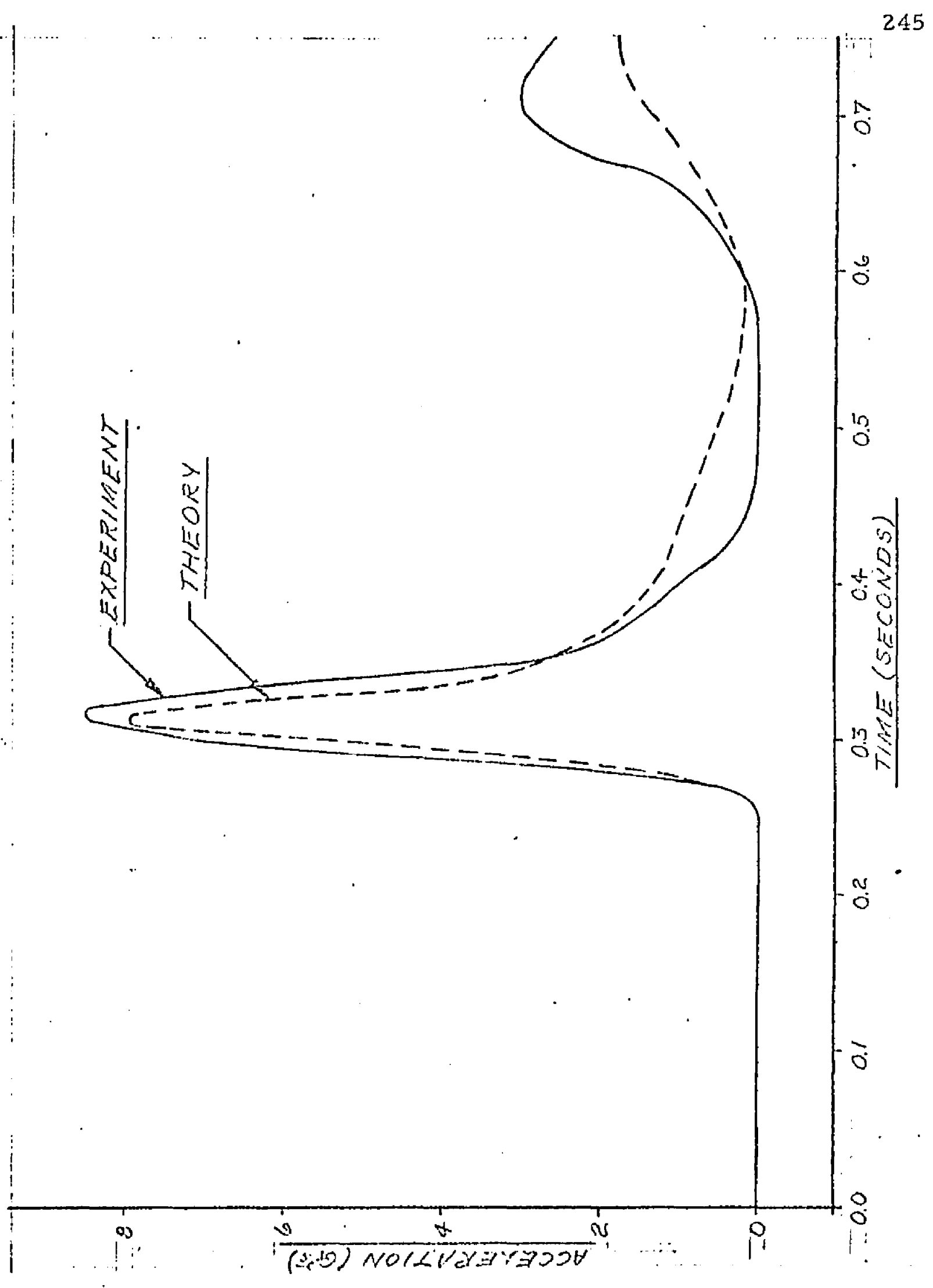

ACCELERATION DURING DROP TEST FIGURE 8-12: 


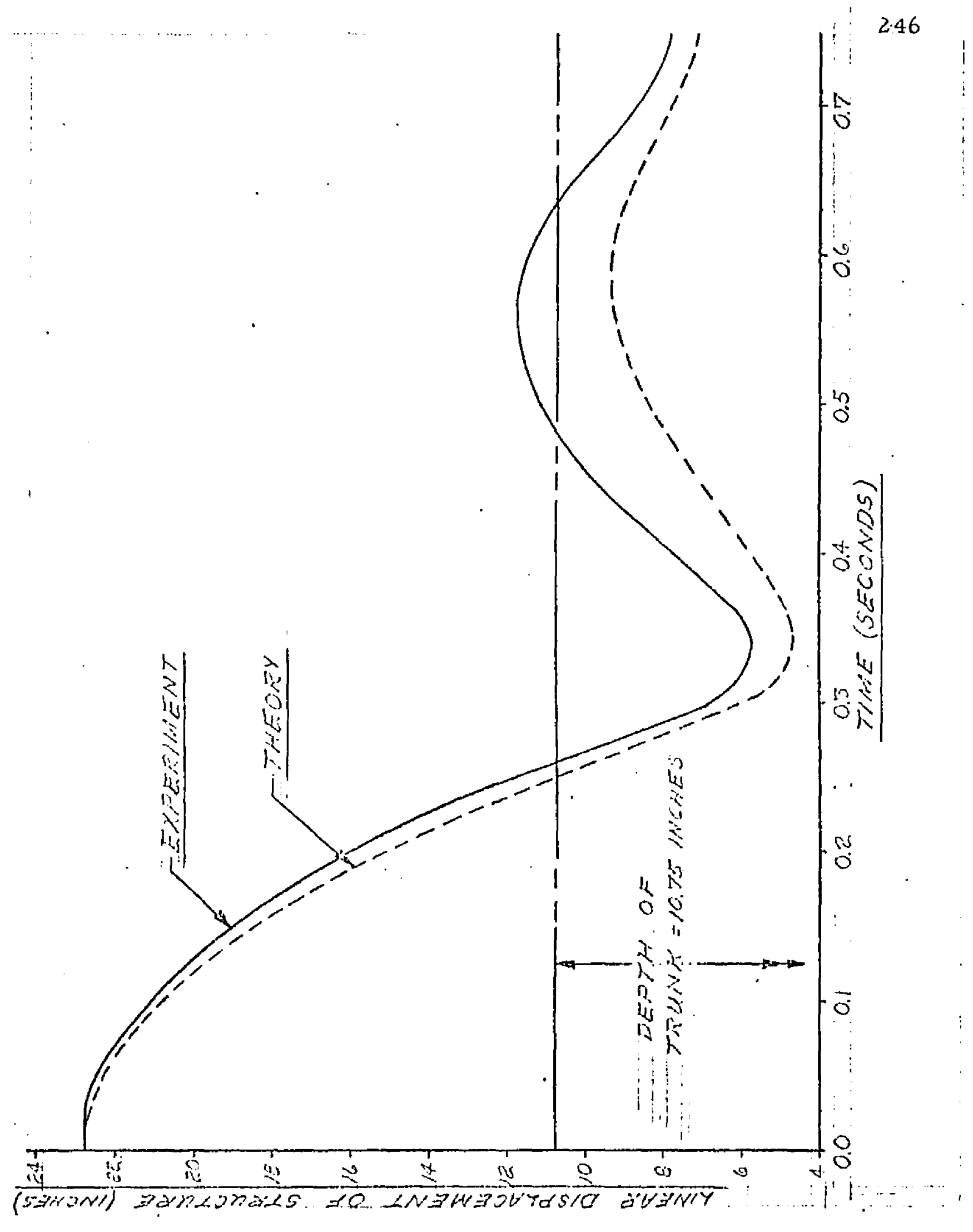

DISPLACEMENT DURING DROP TEST

FIGURE 8-13 


\subsection{Summary of Dynamic Test Results}

The model drop test was conducted in order to compare the experimental results with the computer prediction of the dynamic response.

The fan speed at drop was $8,000 \mathrm{rpm}$ and the drop height was one foot. The experimentally determined static characteristics shown in Figures 8-7, 8-8, 8-9, and 8-10 were used as inputs to the computer program.

The variation of trunk pressure with time for the experimental and the computer results are shown in Figure 8.11 . The shapes of the two curves are quite similar. However, the peak pressure predicted by the computer was higher than that measured.

Figure 8.12 compares the predicted and measured values of the vertical acceleration. As in the case of the pressures, the curves are similar in shape. However, the experimentally measured acceleration was slightly higher than that predicted.

Figure 8.13 compares the predicted and measured values of clisplacement. The curves are similar in sliape, but the maximum predicted displacenent is slightly greater than the measured displacement.

Figures 8.11 through 8.13 show that it is possible to analytically predict the general characteristics of the dynamic response on the model tested. The analysis presented in Section 7.3 represents a valuable design tool for evaluating the effect on dynamic response of changes in the various design variables. 


\section{SUMMARY OF RESULTS}

\subsection{Design Considerations}

In this report, the static and dynamic performance characteristics of the air cushion landing system were considered. The performance characteristics which are associated with static equilibrium include the following:
load capacity
stiffness
obstacle clearance

Additional performance characteristics associated with the dynamic performance of the system include: vertical (landing) energy absorption characteristics, horizontal energy absorption characteristics (braking and frictional drag), and system stability. The horizontal energy absorption and system stability were not considered in this study.

The load capacity is generally a specified design requirement which is determined by the aircraft design.

The system stiffness is dependent upon the trunk shape, trunk pressure, and the configuration of the cushion. It is desired to design the trunk so that pitch, roll, and heave stiffness are adequate. However, it should be noted that trunk stiffness is also an important parameter in designing the air cushion system for landing energy absorption. This consideration may become the overriding factor in specifying the trunk stiffness.

The obstacle clearance is related to the daylight clearance (d), the trunk height $\left(Y_{0}\right)$, and the design of the jets and the trunk. It is generally desired to have large values of $d$ for maximum ground performance but small values of $d$ for minimum power. It is possible, 
through using a flexible trunk with distributed jets, to provide adequate ground performance for low values of $d$. The value of $d$ necessary for adequate ground performance for a given trunk and jet configuration must, at present, be determined experimentally. The resulting value of $d$ is an important variable in determining power requirements for adequate ground performance.

The design variables may be subdivided into the following four areas: aircraft, jet system, trunk, and power system.

The aircraft variables include the weight to be supported $\left(W_{A}\right)$, the length $\left(D_{2}\right)$, width $\left(D_{1}\right)$, area $\left(A_{C}\right)$, and the perimeter $(S)$ of the air cushion, and the daylight clearance (d) between the trunk and the ground. Additional variables which enter into the dynamic performance include take-off and landing speeds, loads and attitudes, vertical velocity at touchdown, braking coefricient and braking distance.

The jet system variables include the type of jets (slots, holes, nozzles, etc.), the jet spacing, the number of jot rows $(M)$, the location of the jet rows on the trunk $\left(\lambda_{n}\right)$, the effective jet thickness $\left(t_{n}\right)$, and the effective jet angle $\left(\theta_{n}\right)$.

The trunk variables include the location of attachment points, $(0,0)$ and $(a, b)$, the trunk length ( $(\ell)$ and the elastic characteristics of the trunk material (E).

The power system variables include the horsepower input (hp) and the pressure $\left(P_{j}\right)$ versus flow $\left(Q_{i}\right)$ characteristics of the fan.

. It is desired to select values for the design variables in such a way that performance requirements are met and the power, weight, and cost of the system are minimized. The design requirements may be specified in terms of aircraft weight, jet height, and trunk stiffness, and maximum allowable deceleration during landing impact.

The relationship between groupings of the design variables are expressed throughout this report in terms of $\mathrm{p}_{\mathrm{c}} / \mathrm{P}_{\mathrm{j}}$. It should be noted that when the aircraft is totally supported by the cushion, $P_{c}$ is completely determined by the supported weight and cushion area. The effect of increasing power is to increase $p_{j}$, which in turn increases jet 
flow. The major effect of increased flow is to increase the jet height, $d$. It is evident that the ratio of $\mathrm{p}_{\mathrm{c}} / \mathrm{p}_{\mathrm{j}}$ is an important parameter which relates the variables of weight, power and jet height. The trunk stiffness and trunk shape are also functions of $p_{c} / p_{j}$. Consequently, the ratio $\mathrm{p}_{\mathrm{c}} / \mathrm{p}_{\mathrm{j}}$ forms an important link between the dependent and independent variables.

In the following sections, the relationships between the various design variables have been summarized."

\section{2 $\quad$ Aircraft Variables}

The principal aircraft variables are as follows:

$$
\begin{aligned}
& A_{c} \text {-cushion area } \\
& D_{1} \text {-cushion width } \\
& D_{2} \text {-cushion length } \\
& \text { d-daylight clearance (jet height) } \\
& \text { S-cushion perimeter } \\
& W_{A^{-a i r c r a f t} \text { weight }}
\end{aligned}
$$

Very little design flexibility is generally allowed in the aircraft variables. The cushion area and shape are generally determined by the aircraft design. Similarly, the weight of the aircraft is specified. The jet height is specified by the obstacle negotiation and ground performance requirements.

A relationship between the principal aircraft variables and the power requirements may be developed by combining Equations (2-9) and (3-7). The result is

$$
h p=\left(\frac{W_{A}}{A_{c}}\right)^{3 / 2} \frac{s d}{550}\left(\frac{2 g_{0}}{\rho}\right)^{1 / 2} C_{h d}
$$

This relationship shows that among the aircraft variables it is desirable to maximize $A_{C}$ and minimize $S$ and $d$ for minimum power. This relationship is further 
illustrated by Figure 3-5 which shows that the augmentation ratio is increased by increasing cushion area for a fixed value of $d$.

A further considoration in designing the air custion system is the pitch and roll stiffness offered by the trunk. It is desired to place the trunk as far from the center of gravity as possible to increase the restoring moment developed by the trunk.

Consequently, it is clesired to make the aircraft fuselage as wide as is permitted by aerodynamic and structural considerations. An optimum cushion shape for an air cushion landing system would probably involve a fuselage with a higher width to length ratio than exists in normal aircraft designs.

9.3 Jet System Variables

The jet system variables include:

d - jet height

$\mathrm{p}_{\mathrm{c}} / \mathrm{p}_{\mathrm{j}}$ - pressure ratio

$\mathrm{N}$ - number of jet rows

$\mathrm{t}$ - total jet thickness

$t_{n}$ - jet thickness for individual rows

$\lambda_{n}$ - location of individual rows on the trunk

$0_{n}$ - effective jet angle for individual rows

In addition, the use of slots versus holes for the jet nozzle must be considered. The diameter and spacing between the holes must be determined if holes are selected.

The selection of $\mathrm{p}_{\mathrm{c}} / \mathrm{p}_{\mathrm{j}}$ is determined largely by the cushion system stiffness and vertical energy absorption desired. Low values of $\mathrm{p}_{\mathrm{c}} / \mathrm{p}_{\mathrm{j}}$ give a stiff cushion while high values of $p_{c} / p_{j}$ give a soft cushion. The influence of $p_{c} / p_{j}$ on power is shown in Figure 3-4la). The power-height parameter $\mathrm{C}_{\mathrm{hd}}$ is directly proportional to power for constant vehicle weight, 
area, perimeter, and jet height. The curves show that power requirements are relatively insensitive to $p_{c} / p_{j}$ for the range of 0.5 to 0.9 . Figures $3-4(b)$ and $3-4(c)$ also show the effect of jet angle on power requirements. An increase in jet angle from $0^{\circ}$ to $30^{\circ}$ results in a considerable decrease in power. Further increases have minor influence on power. Negative jet angles and jet orifices to the outside of the trunk low point $\left(X_{0}, Y_{0}\right)$ were shown in Chapters 5 and 6 to contribute practically nothing to jet height.

The jet thickness $t$ is selected to provide the desired level of $\mathrm{p}_{\mathrm{c}} / \mathrm{p}_{\mathrm{j}}$ for the design weight, jet height, and power setting.

Front and rear trunk sections generally require more jets than side trunk sections. The reason for this may be seen by comparing Figure 4-21 and Figure 4-22. These figures show that for a given deflection, the end trunk has a longer length flattened against the ground $\left(\ell_{3}\right)$ than the side trunk. In addition, the rear trunk undergoes extensive flattening during take-off rotation and landing touch-down. Inadequate air lubrication would contribute to plow-in of the front trunk and excessive wear of the aft trunk.

Because of the complexity of the flow beneath the trunk, an optimun spacing and nozzle design cannot at present be predicted analytically. However, the analysis presented in this report is useful in determining trends and extrapolating experimental results. In particular, the power-jet-height parameter $\left(C_{h d}\right)$ is a valuable parameter for this purpose.

The $\mathrm{C}_{\text {hd }}$ parameter was defined by Equation (3-7) in Section 3.6.

$$
c_{h d}=\frac{t}{d}\left(\frac{p_{j}}{p_{c}}\right)^{3 / 2} \quad c_{O} c_{x}
$$

The values of $t / d$ and $C_{Q}$ as a function of $p_{c} / p_{j}$ may be determined by the simple test described in Chapter 6 on a model section of trunk. The test rig for conducting such a test was shown in Figure 6-1. The test for determining $C_{X}$ was described in Appendix VI. As 
a result of these simple tests, it is possible to plot $C_{\text {hd }}$ versus $p_{c} / p_{j}$ for a particular jet configuration. A comparison of these plots for various jet configurations allows the evaluation of the designs in efficiency of maximizing jet height and minimizing horsepower. It was shown in Equation (9-1) that horsepower is directly proportional to $\mathrm{C}_{\mathrm{hd}}$.

$$
h p=\left(\frac{W_{A}}{A_{c}}\right)^{3 / 2} \frac{s d}{550}\left(\frac{2 g_{0}}{\rho}\right)^{1 / 2} c_{h d}
$$

As a consequence it is desirable to select the design which minimizes $\mathrm{C}_{\mathrm{hd}}$. provided weight, cost, or other factors do not dictate the selection. Other factors include the necessity to provide "air lubrication" beneath the trunk during landing impact, and to stabilize dynamic oscillations of the trunk under all operating conditions.

A comparison of $C_{\text {hd }}$ for two trunk designs is shown in Figure 9-1. In the figure, eight rows of orifices, the design described in Appendix IV, is compared with a design which has four transverse slots. Both designs had the same total nozzle area. The curve shows that the slot design is better for high $\mathrm{P}_{\mathrm{c}} / \mathrm{p}_{\mathrm{j}}$ while the orifice design is better for $10 \mathrm{w} \mathrm{p}_{\mathrm{c}} / \mathrm{p}_{\mathrm{j}}$. It is evident from Figure $9-1$ that the $C_{h d}$ gives a simple vehicle for comparing competing designs without the need for a complicated analysis.

\section{$9.4 \quad$ Trunk Variables}

The trunk variables include:

$(0,0)$ and $(a, b)$ - the trunk attachment points

$\ell$ - the trunk length

E - the trunk material elasticity

$P_{\mathbf{j}}$ - the trunk pressure

The trunk stiffness may be influenced considerably by choosing appropriate trunk lengths and attacinment points. The load supported by the trunk is proportional to $P_{j}$ (the 


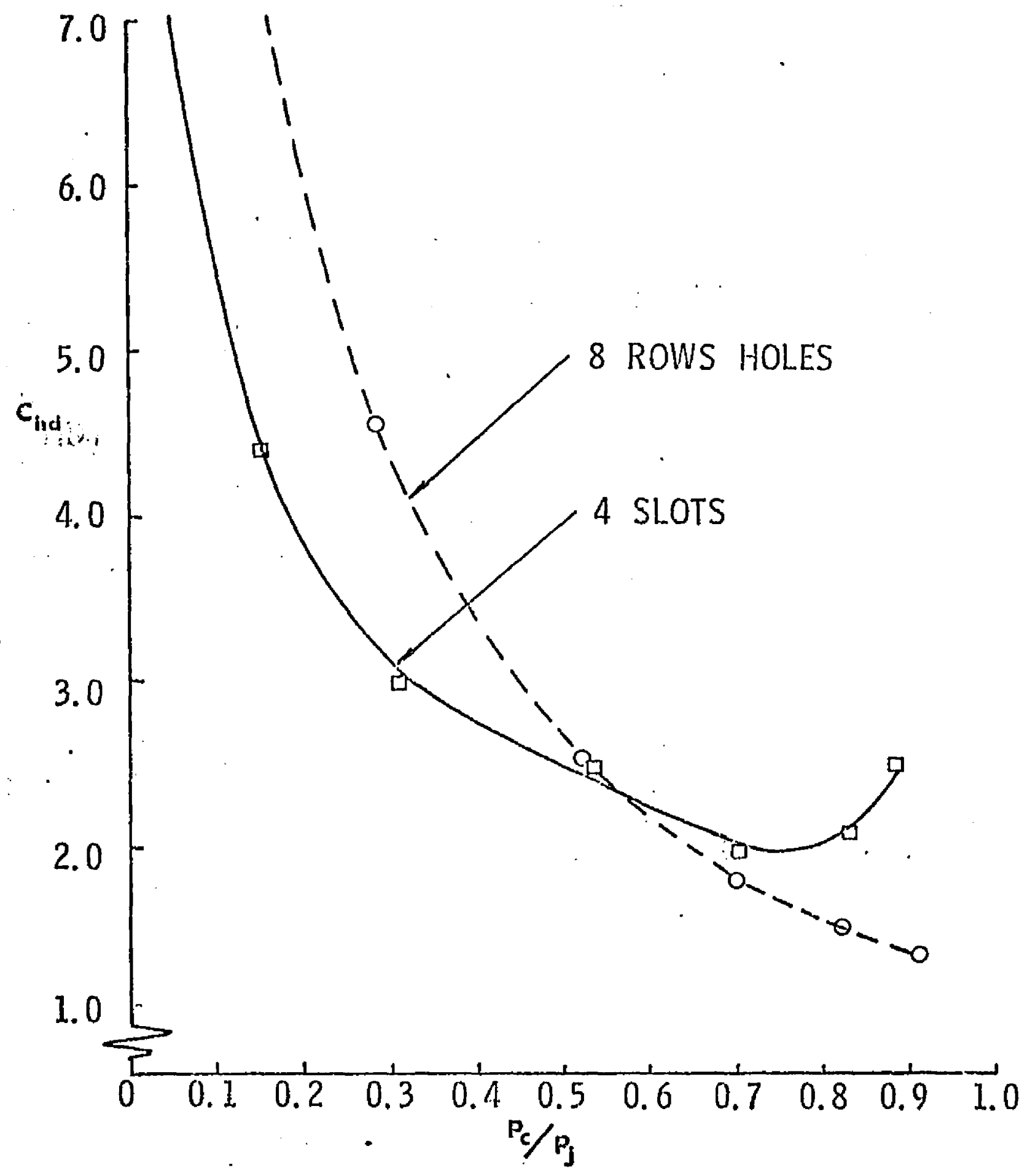

POWER-HEIGHT PARAMETER FOR TWO TRUNIK DESIGNS FIGURE $9-1$ 
trunk pressure) and to $\ell_{3}$ (the length flattened against the ground). The relationships between $\ell_{3}$ and trunk defiection are shown in Figures $4-21$ and 4-22; the two trunks having different attachment points. The stiffness variation, scaled up to a C-119 aircraft size trunk, is shown in Figure 9-2. The curves shown in Figure 4-21 and Figure 4-22 are scaled up to produce curves " $A$ " and " $B$ " respectively in Figure 9-2. The following assumptions were made:

All dimensions are scaled up by a factor of 3.00 .

The design $\mathrm{p}_{\mathrm{c}} / \mathrm{p}_{\mathrm{j}}$ is 0.5 .

The trunk is inelastic.

The design trunk pressure is $333 \mathrm{lb} / \mathrm{ft}^{2}$.

The trunk section is 500 " in length.

The stiffness of the two trunk sections was found to be $2000 \mathrm{Ib} / \mathrm{in}$. for trunk " $\mathrm{A}$ " and $6000 \mathrm{lb} / \mathrm{in}$. for trunk " $\mathrm{B}$ ". The stiffness of the air spring on the conventional C-119 shock s:trut is around $4500 \mathrm{lb} / \mathrm{in}$. It is evident from this simple illustration that considerable flexibility exists in designing trunk stiffness by appropriate selection of the trunk variables. In a manner similar to the illustration above, the stiffness for any trunk design may be calculated from the computer program results.

The selection of the trunk material elasticity is based on the difference between the retracted length and the desired inflated length. It is desirable to have a compound elastic curve with two different slopes. A typical curve is shown in Figure 4-14. The material shown has the slope characteristic of the rubber up to the inflated design point and the slope of the fabric reinforcing material above the design point. Such an elastic characteristic allows the material to stretch easily up to the design point but resists further elongation above the design point.

The analysis of the air cushion trunk shape developed in Chapter 4 and the computer programs developed in Appendices I, II, and III provide the capability of predicting the influence of all the trunk variables on the trunk and cushion stiffness. In 
addition, the dynamic analysis developed in Chapters 7 and 8 provides the capability of evaluating the influence of all trunk design variables except trunk elasticity, on the dynamic response of the vehicle.

9.5 Power System Variables

The power system variables include:

hp - power input

$\mathrm{a}_{i}$ - air flow rate

$P_{\mathbf{j}}$ - trunk pressure

The flow rate is determined from Equation (3-3), which may be written:

$$
\mathrm{Q}_{\mathrm{j}}=\mathrm{ts} \sqrt{\frac{2 \mathrm{~g}_{\mathrm{o}}}{\rho} \mathrm{p}_{\mathrm{j}}} \mathrm{c}_{\mathrm{x}} \mathrm{C}_{\mathrm{O}}
$$

The coefficient $\mathrm{C}_{\mathrm{Q}}$ is a function of $\mathrm{P}_{\mathrm{c}} / \mathrm{p}_{\mathrm{j}}$ and is shown in Figure 3-2.

The power system must be designed so that the desired flow rate is produced at the design $\mathrm{p}_{\mathrm{j}}$. Further, the fan characteristics should be chosen such that the necessary flow will be produced to maintain $\mathrm{p}_{\mathrm{c}} / \mathrm{p}_{\mathrm{j}}$ in an acceptable range over the expected variations of $\mathrm{p}_{\mathrm{c}}$ caused by changes in the aircraft operating weight. Under landing impact, it is possible for $p_{j}$ to increase to the point where the fan stalls and reverse flow occurs. The fan should be designed to permit and withstand this condition.

Consiclering only static conditions, the desired $P_{j}$ versus $Q_{i}$ fan characteristics may be obtained from Equation (3-3). This equation gives the required flow for various levels of $P_{j}$ and $p_{c} / p_{j}$. The value of $C_{Q}$ as a function of $p_{c} / p_{j}$ is given in Figures $3-2,5-7$, and $6-12$ for various cushion designs.

In addition, the fan characteristics play an important role in the dynamic response of the system. The fan flow characteristics near and above stall pressure have a profound 
influence on the maximum trunk pressure and maximum deceleration during impact. The effect of fan characteristics on dynamic response may be evaluated using the dynamic analysis developed in Chapter 7.

9.6 Power Requirements for the ACLS

The power requirements for the ACLS may be scaled up using Equations (9-1) and (3.7).

$$
\begin{aligned}
& h p=\left(\frac{W_{A}}{A_{c}}\right)^{3 / 2} \frac{s d}{550}\left(\frac{2 g_{o}}{\rho}\right)^{1 / 2} c_{h d} \\
& c_{h d}=\frac{t}{d}\left(\frac{p_{j}}{p_{c}}\right)^{3 / 2} c_{Q} c_{x}
\end{aligned}
$$

The $C_{\text {hd }}$ parameter is dimensionless and independent of scale. This parameter may be easily measured for a given trunk design by model testing. The values of $\rho$ and $g_{0}$ are also independent of vehicle size. The remaining variables are dependent on aircraft size and performance requirements. In particular, the value of $d$ is related to the ground performance requirements, and $A_{c}$ and $S$ are related to aircraft weight. $A 2500$ pound aircraft equipped with an air cushion landing system has been tested and its take-off, landing and obstacle negotiation performance was excellent as reported in References (3) and (50). If it is assumed that the jet height and $\mathrm{C}_{\mathrm{hd}}$ of the test aircraft design are satisfactory for larger aircraft, the power requirements for larger aircraft may be estimated from Equation (9.1).

To determine the relationship between the power and aircraft weight, some dependence between weight and fuselage area, and weight and fuselage perimeter, is necessary. 


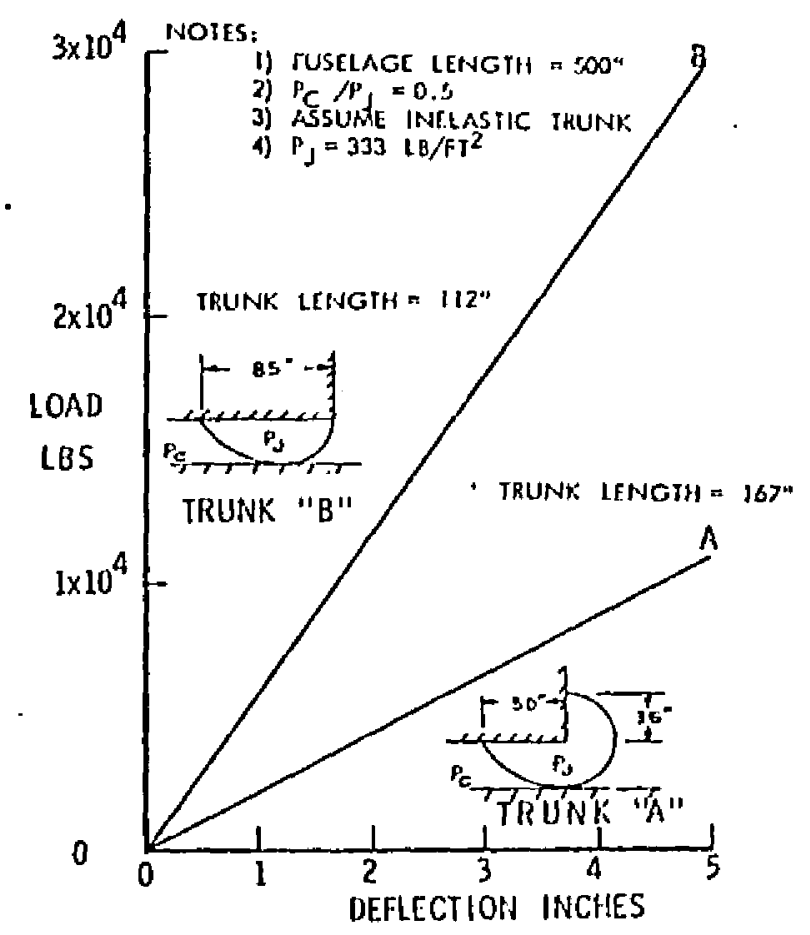

LOAD DEFLETION CHARACTERISTICS FIGURE $9-2$

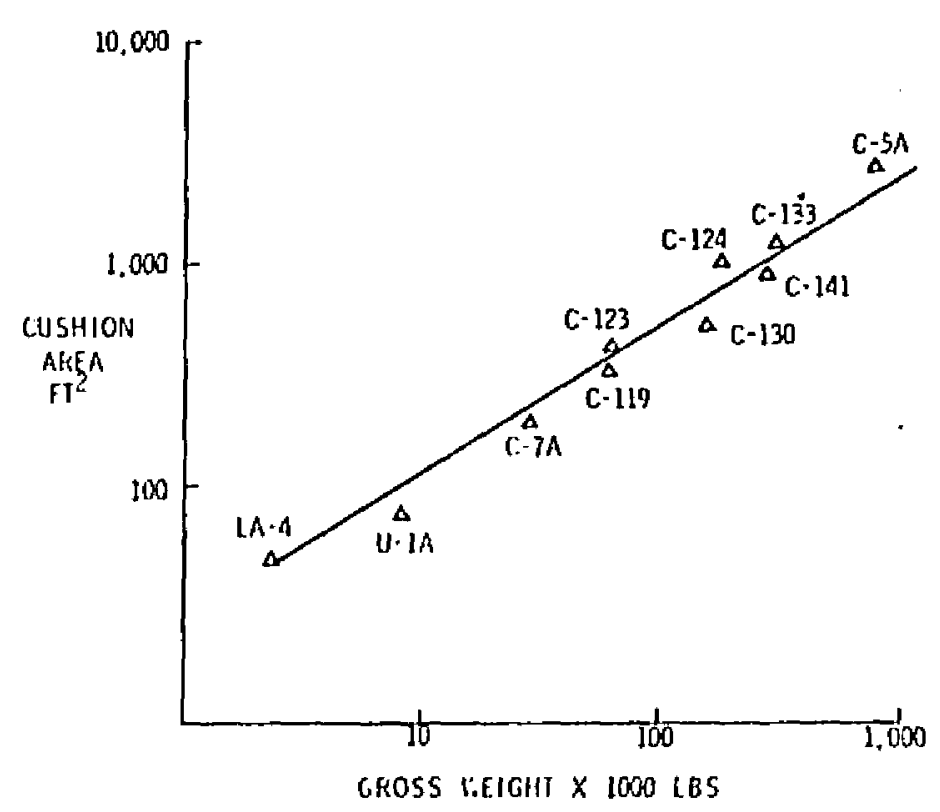

FUSELAGE AREA VS A/C GROSS WEIGHT

FIGURE $9-3$

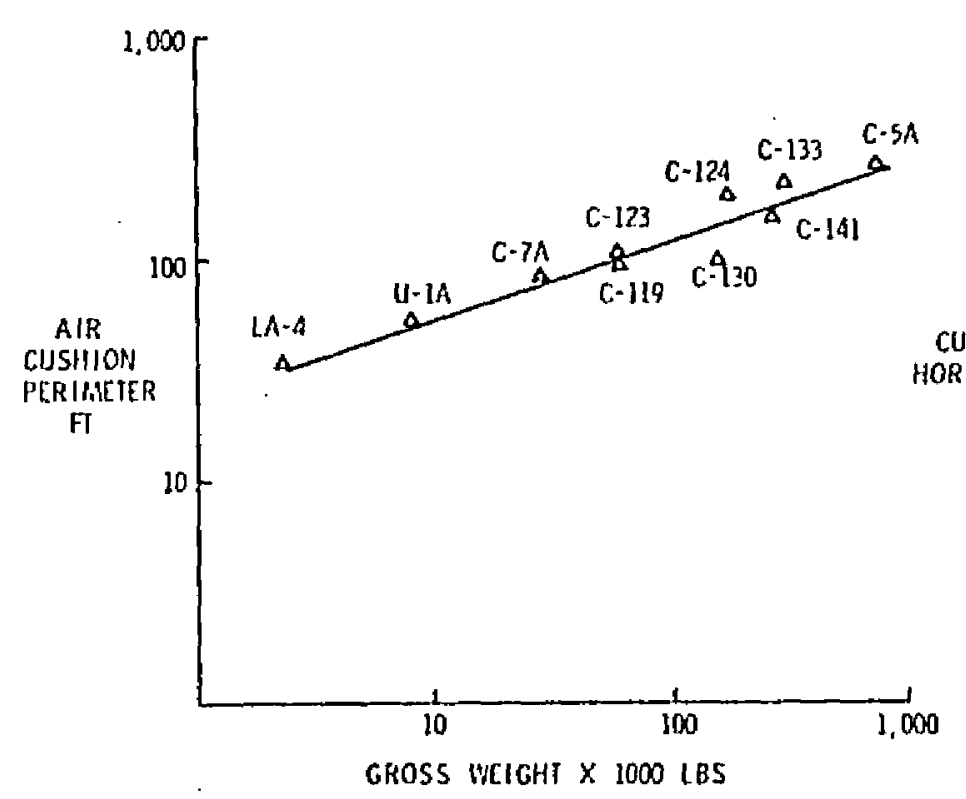

fuselage PERIMETER VS A/C GROSS WEIGHT FIGURE $9-4$

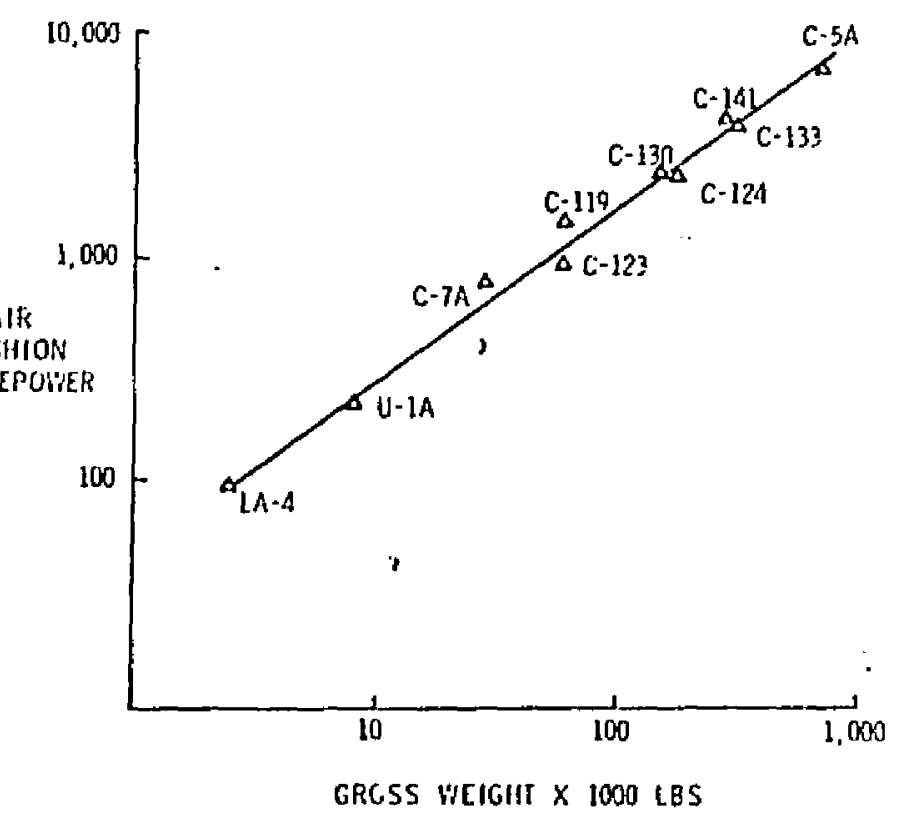

AC LS POWER VS A/C WEIGHT FIGURE $9-5$ 
Figure 9-3 shows a plot of fuselage area versus weight for various cargo and utility aircraft. A similar plot of fuselage perimeter versus aircraft weight is shown in Figure 9-4.

Using the relationships of Figures 9-3 and 9-4 in Equation (9-1), it is possible to estimate ACLS horsepower as a function of aircraft weight. The resulting power requirements are shown in Figure 9-5. A plot of the installed propulsive horsepower for the aircraft is shown for comparison.

It should be noted that the results in Figure 9.5 assume a constant jet height and neglect the effect of compressibility and ducting losses. Figure 9-5 shows that ACLS power requirements are proportional to $\left(W_{C}\right)^{5 / 6}$. At aircraft weights in the 60,000 pound class, approximately $20 \%$ of the propulsive power would be required. At weights in the 600,000 pound class, only $15 \%$ would be required. It is evident that the power required by the ACLS is only a small fraction of the normal propulsive power and an even smaller fraction of the power required for vertical takeoff. The ACLS offers the aircraft remarkable improvements in ground performance for a modest increase in power.

\section{$9.7 \quad$ Conclusions}

As a result of the work reported herein the following conclusions are made:

(1) The cross-sectional area and shape of an air cushion trunk of the general configuration tested (Chapter 6) can be analytically predicted using the analysis in Chapter 4. The agreement between theory and experiment was yood for both the free and ground loaded cases.

(2) The classical peripheral jet momentum theories (Chapter 2) do not adequately predict the jet height and flow for a distributed jet of the type used on the aircraft in the air cushion landing system flight test program. $(3),(50)$ 
(3)

The flow restrictor theory developed in Chapter 5 gives excellent agreement with experimental results reported in Chapter 6 for jet height, flow and pressure distribution around the trunk for the trunk configuration tested.

(4) The presently used orifice system is inefficient from the standpoint of jet height. As far as the jet height is concerned, the momentum from the jet exhaust and the flow from the jets on the atmospheric side of the trunk low point are almost totally wasted.

(5) The dimensionless parameter $C_{Q}$ provides an accurate compensation for the effect of $p_{c} / p_{j}$ on the total flow from the trunk.

(6) The parameter $\mathrm{p}_{\mathrm{c}} / \mathrm{p}_{\mathrm{j}}$ was found to be a valuable dimensionless quantity for relating the various dependent and independent variables. Test results reported in Chapter 6 showed that both jet height $d$ and $C_{Q}$ were dependent on $p_{c} / p_{j}$ and relatively independent on the magnitude of $p_{j}$ alone.

(7) The trunk shape analysis developed in Chapter 4 for a trunk with free edges gave good agreement with experimental results when applied to the complicated dynamic test model reported in Chapter 8.

(8) The dynamic analysis developed in Chapter 7 gave good agreement with the dynamic test reported in Chapter 8 for a drop test with $p_{c}=0$. 
(9) The trunk shape analysis developed in Chapter 4 provides the capability of analytically evaluating the effect of trunk length, attachment points, material elasticity, cushion pressure and trunk pressure on trunk shape, volume and stiffness.

(10) The flow analysis developed in Chapter 5 provides the capability of analytically evaluating the effect of jet size, spacing, angle, position on the trunk, cushion pressure, trunk pressure, and trunk shape on the resulting jet height and flow.

(11) The dynamic analysis developed in Chapter 7 provides the capability of anatytically evaluating the influence of aircraft weight, sink velocity, fan characteristics, trunk shape, trunk length, and trunk orifice area and spacing on the dynamic response of the vehicle under landing impact.

(12) The dimensionless parameter $C_{h d}$ is a valuable vehicle for comparing the relative effectiveness of competing designs for minimizing horsepower and maximizing jet height. The value of $\mathrm{c}_{h d}$ for a design may be determined easily by test, thereby eliminating a complicated analysis. The parameter $C_{h d}$ is also valuable for scaling model test results to full size vehicles.

(13) The air cushion landing system offers a promising area for further development. 
Appendix 1

FREE TRUNK SHAPE (INELASTIC)

The computer program described in this appendix computes the cross-sectional shape for a free inelastic trunk. The logic is similar to that presented in Section 4.4, but with the restriction that the trunk is inelastic.

The input variables are

$$
\begin{array}{ll}
\mathrm{a} & =\mathrm{x} \text { coordinate of upper trunk attachment point } \\
\mathrm{b} & =\mathrm{y} \text { coordinate of upper trunk attachment point } \\
\mathrm{p}_{\mathrm{c}} / \mathrm{p}_{\mathrm{j}} & =\text { ratio of cushion pressure to trunk pressure } \\
\ell & =\text { trunk length }
\end{array}
$$

The program uses $a$ and $b$ to make an initial estimate $R_{1}$ and computes $\bar{l}=$ $\bar{l}\left(R_{1}\right)$. Improved estimates on $R_{1}$ are made until $|\ell-\bar{l}|$. >(TOL)( $\left.\ell\right)$. TOL is the relative tolerance on $\vec{l}$. This tolerance is set at $3 \times 10^{-5}$. This can be changed by inserting a new card.

The main program may call three subroutines: function $F\left(R_{1}\right)$ evaluates $\bar{l}\left(R_{1}\right)-$ $\ell \equiv F\left(R_{1}\right)$; function $D F\left(R_{1}\right)$ evaluates the derivative of $F\left(R_{1}\right)$; subroutine $R T M I$ uses Muelter's Iteration Method to converge on the solution of $F\left(R_{1}\right)=0$, once the solution is bounded.

Initially, the program converges on the solution of $F\left(R_{1}\right)=0$ from the right side using Newton's itcration method.

If the solution is bounded during the Newton iteration process, the Mucller subroutine is called to speed convergence, and a notation is made in the clata output to indicate that this subroutine was used. 
The program has been found to converge for the range of variables which are of practical interest. For extremely small values of $\ell$ (say $\ell \approx \sqrt{a^{2}+b^{2}}$ ), an improved initial guess on $R_{1}$ is necessary. This may be done by inserting a card in the location noted in the program. The variable $\mathrm{p}_{\mathrm{c}} / \mathrm{p}_{\mathrm{j}}$ is restricted to values less than 1.0 .

The output gives the values of all input variables, the notation Mueller if the RTMI subroutine was called, and the final values of the following variables: $A_{j}, R_{1}, R_{2}, X_{0}$, $y_{0}, y_{1}, y_{2}, o_{1}$, and $a_{2}$. 
DIGGES

EQTRSH - EFN SOURCE STATEMENT - IFN(S) -

$12 / 01 / 69$

COMMON /CO/ $A, B, P C P J, L$

COMHON IOERI CI,C2,YO,XO,YL,Y2,THI,TH2,SGN

REAL LI,L2,L,LO,LN, LHH I,L3

DATA Pl $/ 3.1415927$

EXTERNAL F

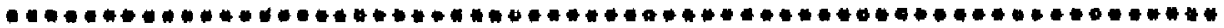

TOL IS A RELATIVE TOLERANCE ON LBAR - CAN BE CHANGED BY

IISERTIHG CIIRD.

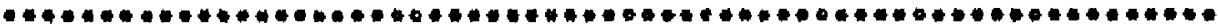

TOL $* 3 . E-5$

1 READ $(5,10) A, B, P C P J, L$

10 FORHAT $\{$ AE20.4)

HRITE $(6,11) A, B, P C P J, L$.

12 FORHATIIHO///4H AM, E16.4,10X,4HB $=, E 16.4,10 X, 8 H P C / P J=, E 16.4$,

$110 \times, 4 \mathrm{HL} \#, E 16.41$

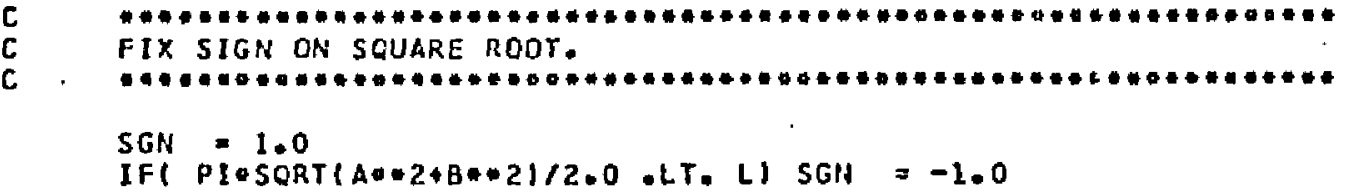

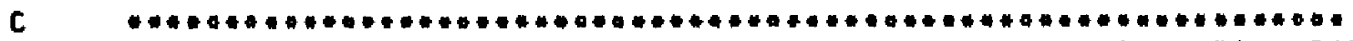

C RO EQUALS INITIAL GUEES FOR RL. CAIA BE CHANGED BY INSERTING CARD.

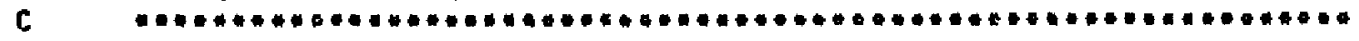

$R O=\operatorname{sonT}(A * 2+B \cdot 2) *(1.0+10 . *+(-6)) / 2$.

RN $=$ RO

$c$
$c$
$c$

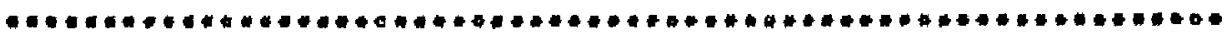

CALCULATE K-TH VALUE OF R AND OBTAIN LBAR (R)

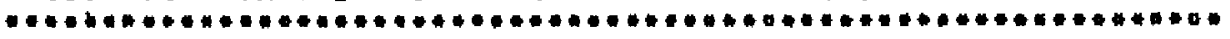

D0 $68 K=1,1000$

C

C SUBACUT INE F COMPUTES LDAR -L

C

$2 \quad P L N=F(R N)$

$L N=P L N+L$

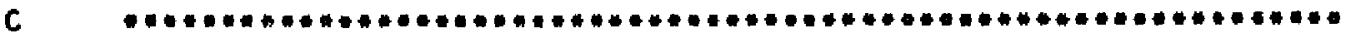

C IS R NEGATIVE OR IS LOAR (R) COMPLEX. IF SO $R(K+1)=(R(K+1)+R(K)) / 2$

C (THIS OCCURS HHEN R(K) IS TOO SHALL)

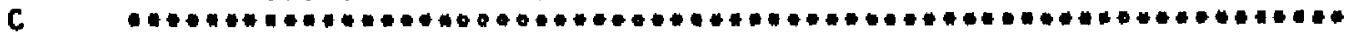

IFIPLN -HE. 10.0*15, AHO. RN, GT. 0.) GO TO 4

$\because \quad$ IFI $K \cdot$ EO. I) GO TO 70

RN $=(R N+R N M I) / 2.0$

GO TO 2 
DIGRES

EOTRSH - EFN SOURCE STATEHENT - IFN(S) -

$12 / 01 / 69$

4 IFIK. EO, II 60 TO 5

C

C CETERHINE IF SOLUTION HAS BEEN BOUNDED. IF SO SET BOUNDS

C ATID CALL MUFLLER ROUTINE. IF NOT COMPUTE R(K+1) USING

C MEHTCA'S FORMULA.

ITI SIGN(1, L-LH) .NE. SIGH(1,LLLHHI)IGO TD 100

$5 \quad L N M=L N$

C

C SUBRCUT INE OF CUMPUTES LBAR'(R)

C

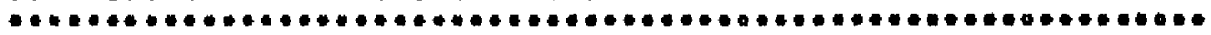

CLN = DF(RI:)

$R 1=R N$

IF( ABS(LN-L) \&.T. TOLAABSIL)I GO TO 110

RNM $1=$ RN

RN $=R N-I L N-L I / D L N$

68 CONTINUE

70 HRITE $(6,71)$

71 FORMATIITHI RO COMPLEX .... STOP

100 IFI RN,GT. RNHII GO TO 105

DUM = RII

RN = RNMI

RNML $*$ OUM

105 WRITE $(6,104)$

104 FORKAT (1HO, THHUELLER)

CALL RTHI I RI, LN,F, RNML, RN, TOL ,2000,IERI

IFC IER . EQ. OI GO TO 110

WRITE $(6,106)$ IER,RI,LH

106 FORMAT(1HL, 2OHIER EOUAL , I2,5X,4HSTOP, 2E25.6)

STOP

$110 \mathrm{R} 2 \Rightarrow \mathrm{R} 1 /(1 .-\mathrm{PCPJ})$

$X 07=X 0 / A$

$L 1=R I * T H 1$

$L 2=R 2+T H 2$

$L 3=0.0$

HRITE $(6,55) R 1, R 2, X 0, Y 0, Y 1, Y 2, T H 1, T H 2$

55 FORMAT (1HO,5HR1 $=, E 16,4,10 X, 5 H R 2=, E 16,4,10 X, 5 H X 0=, E 16.4,10 X$,

1 5HYO $=. E 16.4, / 1 X, 5 H Y 1=, E 16.4,10 X, 5 H Y 2=, E 16.4,10 X$,

2 GHTHL $=$ E15.4, 10X, GHTH2 $=$,E15.4 $/ 1 / 1 / 1 / 1 / 1$

GO TO 1

END 
- EFN SOURCE STATEMENT - IFN(S) -

$12 / 01 / 69$

FUNCTION F IRI)

COMMCN /CO/ A, B,PCPJ,L

COMMON /DER/ C1,C2,YO,XO,YL,Y2,TH1,TH2,SGN

DATA PI, 3.1415927

$c$
$c$
$c$
$c$

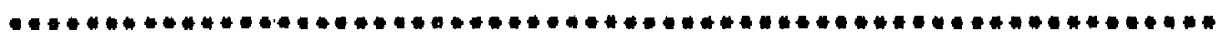

IF $R(K)$ IS SUCH THAT L-BAR HILL BE CPHPLEX, THE VALUE

CF 1 . LBAT: L IS SET TOS IOUA 15

REAL L

$R 2=R 1 /(1.0-P C P J)$

$C 1=(R L-B-R 2), A$

$C 2=A / 2.0+(B . * 2) /(2.0 . A)-(R 1.0) / A$

$A S O=(2.0 * R 2+2.0 *(1 . C 2) * 2-(4.0 * C 2 * 02) *(C 1 * 2+2+1.0)$

IFI $\triangle 5 Q$.LT. 0.0 I GO TO 25

SO. SCRT (ASO)

$Y O=(-2.0 \cdot(R 2+C L \cdot C 2)+S G N+S O) /(2.0 \cdot(C) \cdot-2+1.0)\}$

$X_{0}=C_{1} * Y_{O}+C_{2}$

$Y 1=Y O+R 1$

$Y 2=Y O+R 2$

THZ - MTAN(XO/YZ)

IFI Y2.EQ. 0.1 THL $\times P 1 / 2.0$

IFITHZ :LT O O.OI IHZ = TH2 +PI

PSI $=A T A N(1 B-Y) \mid /(A-X O))$

IFI $A-X 0120,23,21$

20 PSI $=P S I+P I$

$21 T H 1=P S I+P I / 2.0$

$F=R 1$ THL $+R 2 * T H 2-L$

C

C IF VALUE OF VARTABLES OH EACH ITERATION IS DESIRED, REMRVE

C C ON THE THO WRITE STATEMENTS.

C NRITE $(6,22) R L, R 2, T H L, T H 2, Y O, A S Q, C L, C 2, P C P J, X O, Y 1, Y 2, A, B, F$ RETURN

23 PSI $=P I / 2.0$

GO TO 21

$25 \quad F=10.0 * 015$

C HRITE $(6,22) R L, R 2, T H 1, T H 2, Y, O, A S Q, C 1, C 2, P C P J, X O, Y L, Y 2, A, B, F$

22 FORMATIIHO/ (TE1B.5)

RETURN

END 


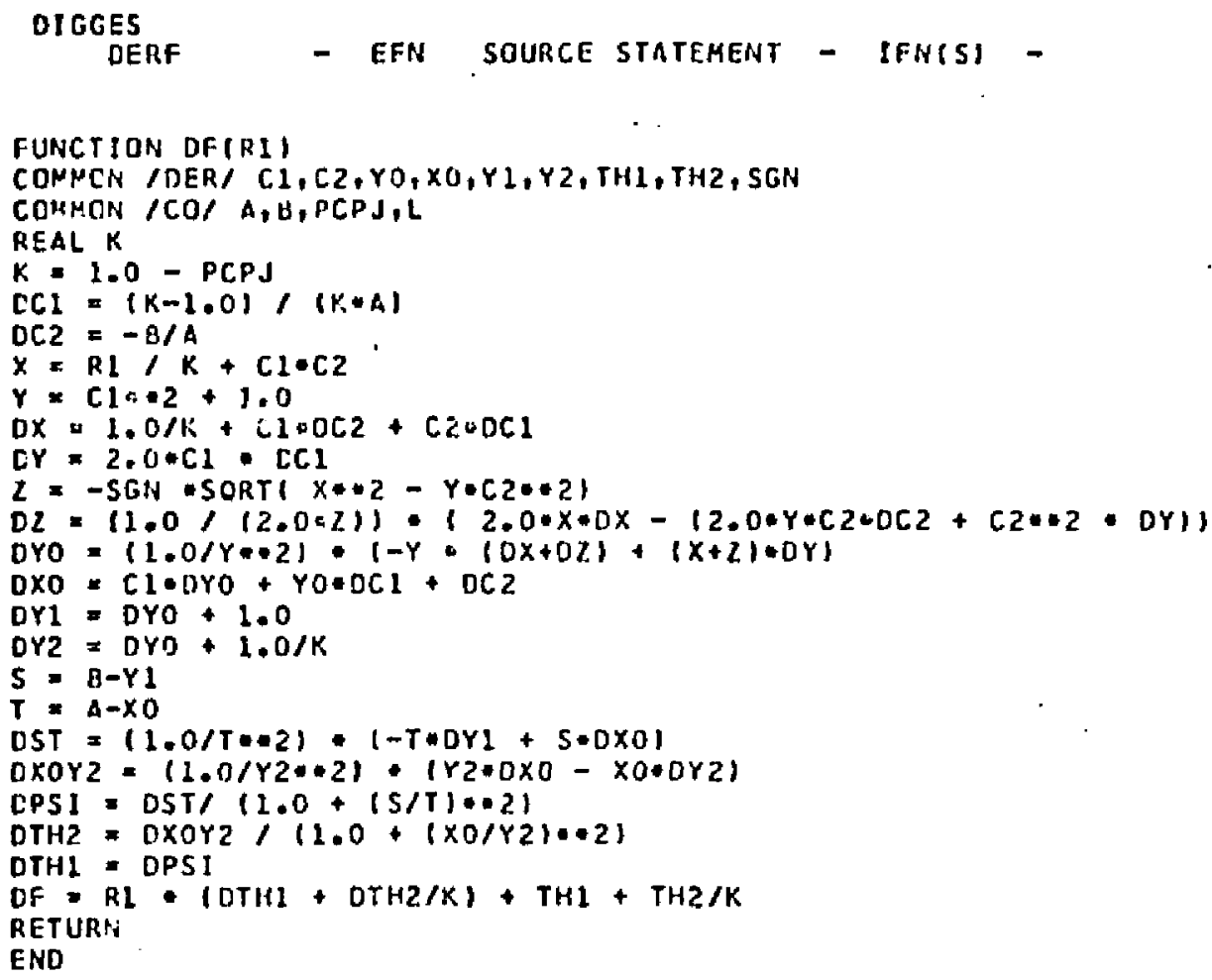


คTH I - efr squrce statehent - ifn(s) -

$06 / 09 / 69$

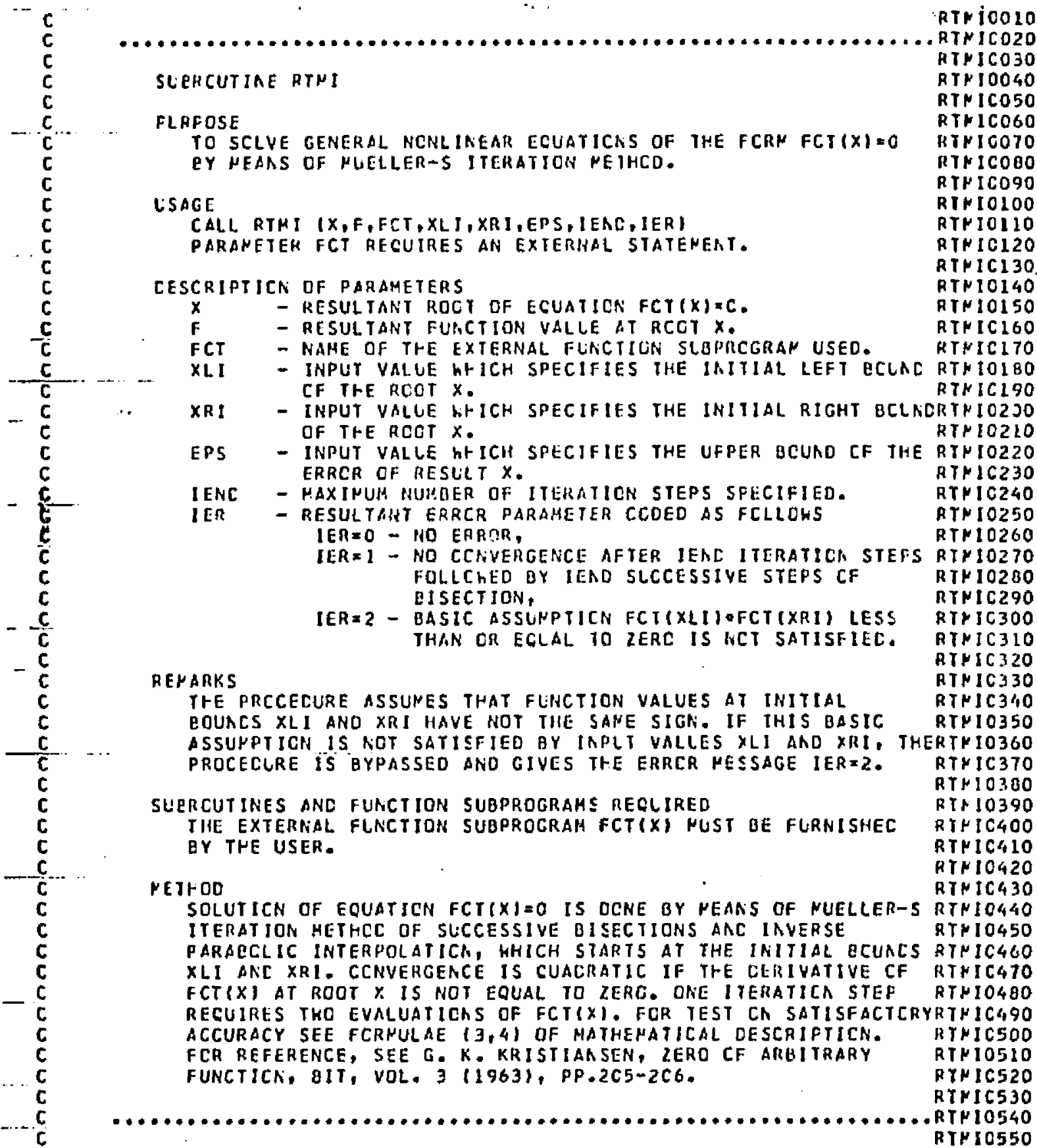




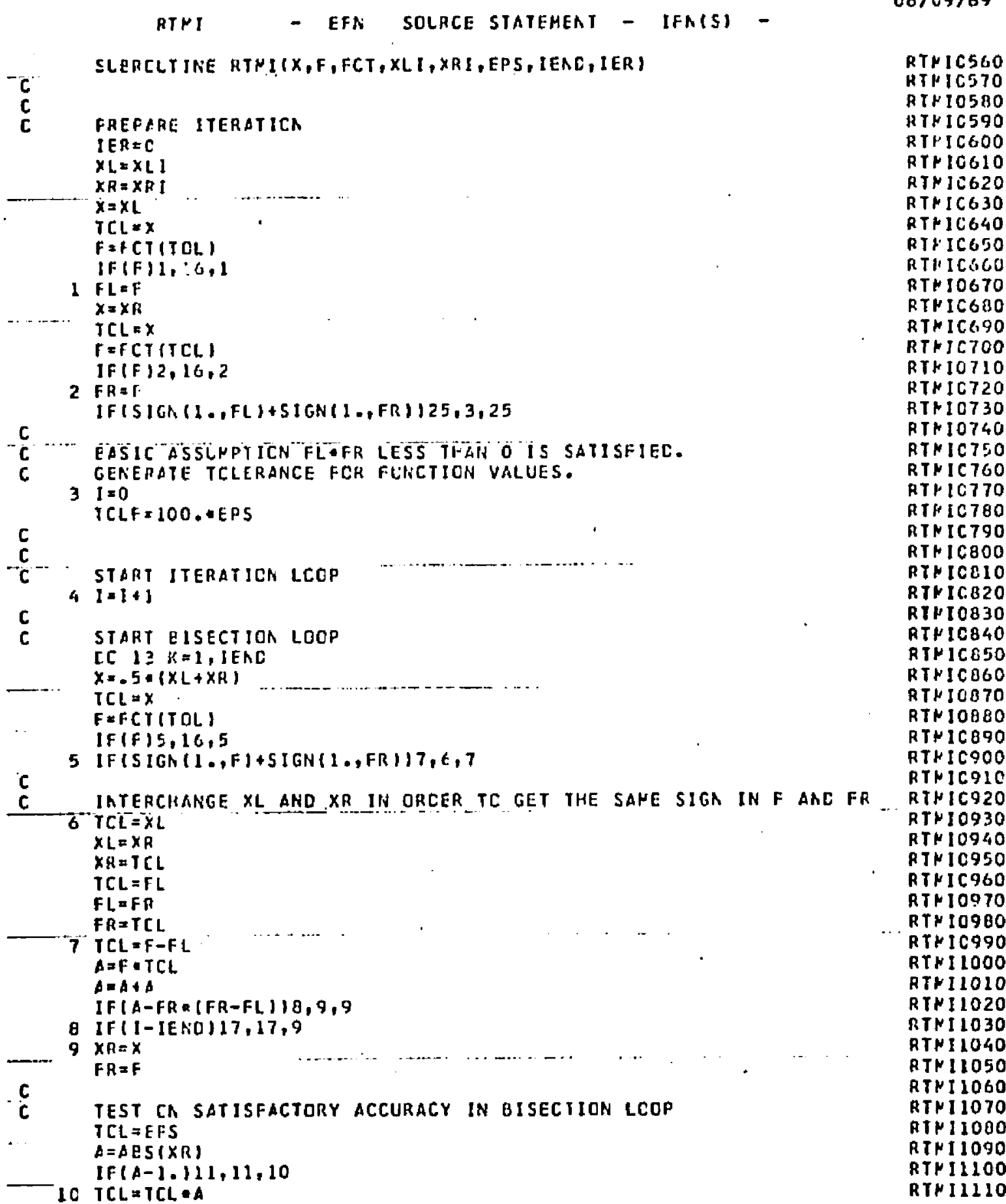$$
\text { c }
$$ 
$\stackrel{ }{N}$

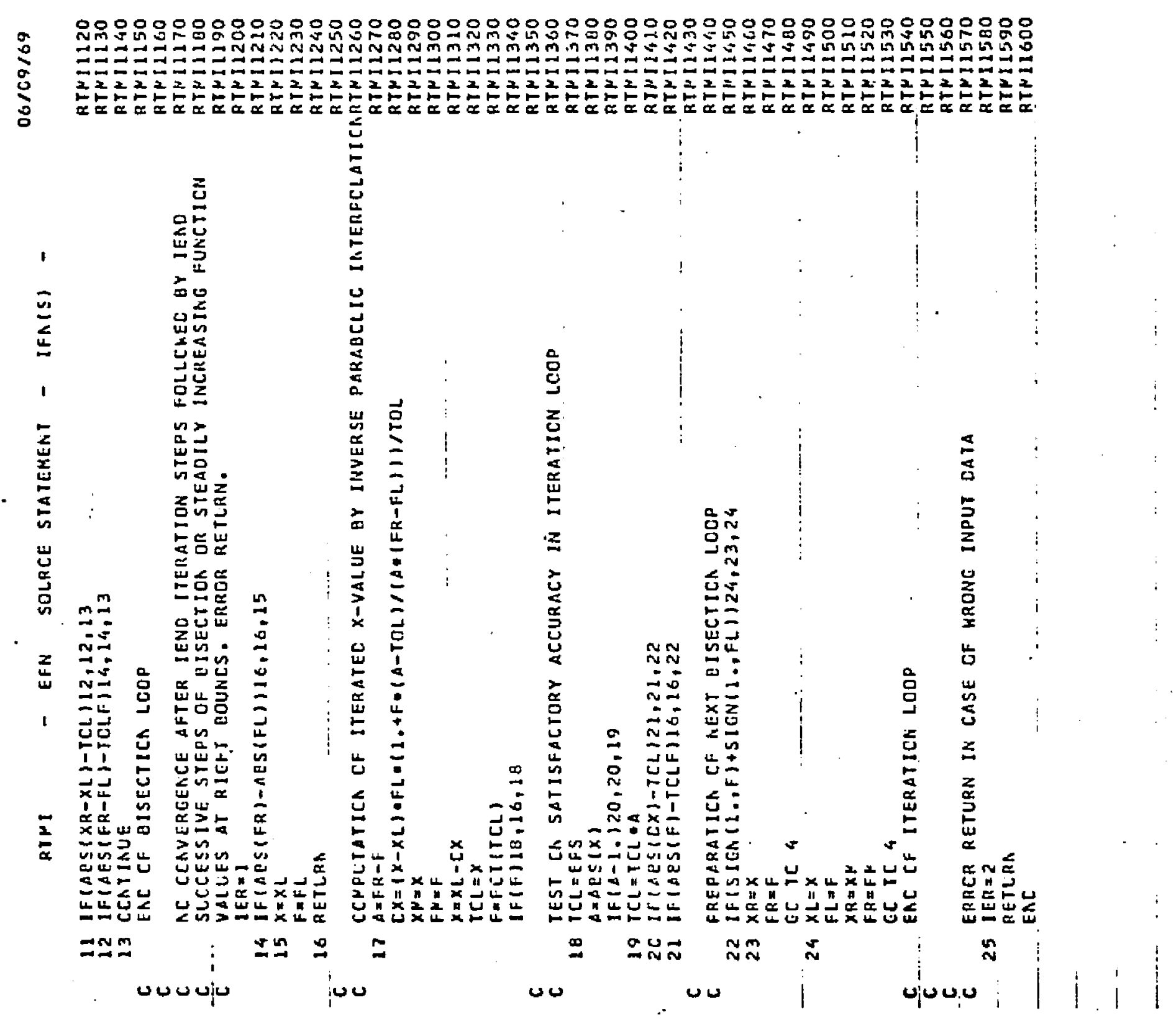




\section{Appendix II \\ INELASTIC LOADED TRUNK SHAPE}

The computer program described in this appendix computes the cross-sectional loaded shape for an inelastic trunk. The logic is similar to that presented in Section 4.5 , but with the restriction $E=0$.

The input variables are:

a $=x$ coordinate of the upper trunk attachment point

b $\quad=y$ coordinate of the upper trunk attachment point

$\mathrm{p}_{\mathrm{c}} / \mathrm{p}_{\mathrm{j}}=$ ratio of cushion pressure to trunk pressure

$\ell=$ trunk length

$y_{0}=y$ coordinate of lower-most segment of the trunk. (Note: $y_{0}$ is always negative.)

The program solves Equation (4-37) $\left(\bar{Q}\left(R_{1}\right)-\ell=0\right)$ to the desired tolerance using Mueller's Iteration Method. The main program brackets the solution and then calls the Mueller subroutine. The Mueller subroutine may call Function $F\left(R_{1}\right)$ or Function $G\left(R_{1}\right)$.

The subroutines are as follows:

Subroutine RTMI uses Mueller's Iteration Method to converge on the solution of $F\left(R_{1}\right)=\bar{\ell}\left(R_{1}\right)-\ell=0$.

Note: This is the same subroutine as described in Appendix 1 and it is not repeated here.

Function $F\left(R_{1}\right)$ evaluates $\bar{l}\left(R_{1}\right)-\ell=F\left(R_{1}\right)$. 
Note: This is not the same $F\left(R_{1}\right)$ subroutine as described in Appendix I because $\ell\left(R_{j}\right)$ is defined differently in the two cases.

Function $G\left(R_{1}\right)$ evaluates $x_{1}\left(R_{1}\right)-x_{2}\left(R_{1}\right)=G\left(R_{1}\right)$.

The input variables $b$ and $y_{O}$ are used to compute $\left(R_{1}\right)_{M I N}$, the minimum value of $R_{1}$ which is pussible. This value of $R_{1}$ gives the condition $x_{1}=a$. The $\bar{l}$ associated with the minimum $R_{1}$ is then computed. This value of $\bar{\ell}$ is called $\ell_{4}$ and is used in determining the sign on the square root in Equation (4-36). Three possibilities exist:

Case $1-$ If $\ell>\ell_{4}$ then $x_{1}>$ a and the sign is plus

Case $2-$ If $\ell=\ell_{4}$ then $x_{1}=$ a and the radical is zero

Case $3-$ If $\ell<\ell_{4}$ then $x_{1}<$ a and the sign is minus

For cases 1 and $2,\left\langle R_{1}\right\rangle_{M I N}$ is a suitable lower bound for the solution of $F\left(R_{1}\right)=$ 0 . The upper bound is found from Equation (4.40). Once the upper and lower bounds are established the Mueller subroutine is called to converge on the solution.

For case 3 , it is necessary to find a lower bound on the equation $F\left(R_{1}\right)=\ell\left(R_{1}\right)$ $-\ell=0$. The minimum $\ell\left(R_{1}\right)$ occurs when $\ell_{3}=0$ and $x_{1}=x_{2}$. The equation $G\left(R_{1}\right)=$ $x_{1}\left(R_{1}\right)-x_{2}\left(R_{2}\right)=0$ is solved by Mueller's method to determine the value of $R_{1}$ for the condition $x_{1}=x_{2}$. The upper bracket for $G\left(R_{1}\right)=0$ is taken at $x_{1}\left(R_{1}\right)=a$. The lower bracket for $G\left(R_{1}\right)=0$ is taken as $x_{1}\left(R_{1}\right)=0$. Function $G\left(R_{1}\right)$ is called by the Mueller subroutine in this case. The $R_{1}$ obtained from $G\left(R_{1}\right)=0$ equation is then taken as the lower bracket for the $F\left(R_{1}\right)=0$ equation. The upper bracket for $F\left(R_{1}\right)=0$ is taken as $x_{1}\left(R_{1}\right)=a$. Having bracketed the solution for $F\left(R_{1}\right)=0$, Mueller's Iteration Method is employed to converge on the solution. 
The program has been found to converge for the range of variables of practical interest. Restrictions are as follows:

$$
\begin{aligned}
& \mathrm{p}_{\mathrm{c}} / \mathrm{p}_{\mathrm{j}}<1.0 \\
& \mathrm{y}_{\mathrm{o}}<0 .
\end{aligned}
$$

$y_{0}$ must be such that $\ell_{3}>0$. The maximum value of $y_{0}$ is given by the equilibrium trunk shape program (Appendix I).

The output gives the initial values of all input variables and the final values of the following variables:

$$
R_{1}, R_{2}, 0_{1}, \theta_{2}, y_{1}, y_{2}, l_{1}, l_{2}, \bar{X}, A_{j}, l_{3}, x_{1} \text {, and } x_{2} .
$$

The final value of $F\left(R_{1}\right)$ is also printed under the lable $L N$. 
DIFAFN - FFH SNLRCE STATEMENT - IFN(S) -

COMWONICCN,OCOJ, Y R,L,A,R,THI,THZ,XI,X2,YI,Y2,PSI, SIGN, T,L4

QFAL $L, L h, L A, L 3, L], L$ ?

EXTERNAL $F, r_{1}$

$P I=3.14155 ? 7$

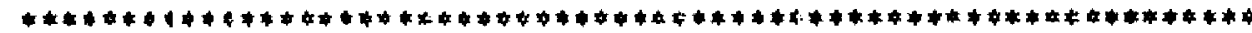

TIL IS A RELATIVE TILEPANCE ON LRAH. CAN AE CHANGED BY

INSERTIHI; $C A R$.

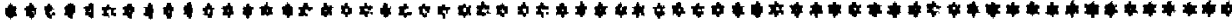

$T O L=3 \cdot E-E$

105 REAC(5,1) $\triangle, 9, D C O \mathrm{~J}, 1, Y O$

1 FORNAT (5E!:.4)

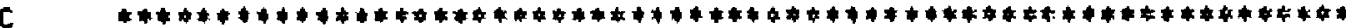

C. RO FOIJALS INITIAL GUESS FOR RL. CAN BE CHANGED BY INSERTING CARD.

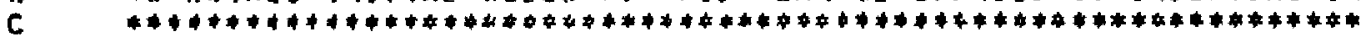

$R 0=(A-10) *(1.0+2 C .0 *(-6)) 12.0$

HRITE

2 FCRNATI 1 - 11

RNMT =

$\operatorname{AMAX} 1(-Y O *(1 .-\rho C P J) / 2,(B-Y O) / 2$.

C $\quad$ F

FIX SITIN CN SOIIARE RONT.

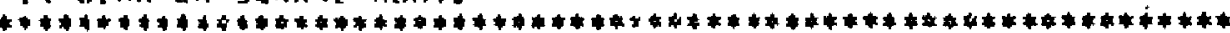

$S I G N=1$.

C

SHBROUTINE F IS CALLED TO DETAIN L 4

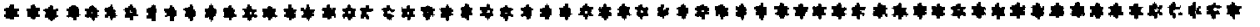

$T=F(P \cap N \mid)$

1.......

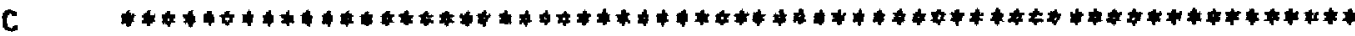

$C$ DETERMIRE HHETHER $X 1$ IS GREATER OR LESS THAN A.

C IF GREATEA SIGN IS DOSITIVE

C IF LESS SIEA IS NEFATIVE

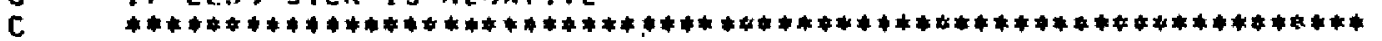

IFI L.LT. LAI GO TO 10C

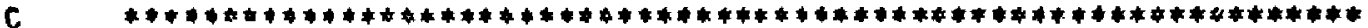
CONDITICN $X 1$ GT A. CCMPUTE LPPER BOUNC CN R.

C

$S O R=S C R T(t * * 2+R * * 2)$

$R N=L / 12.0 * P I 1+S O R / 4.0$

IFISNR :LE. $2,4 L / P I$ T RO TO 3

$R N=L / P I$

Dก $5 \quad 1=1,30$

$R N=10,1 \mathrm{RK}$

IFIRN $\operatorname{SIN}(L / 2 . * R N), G E$ SOR/?.) GO TO 3 
DITINR - EFN SMURCE STATENENT - IFA(SI -

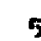

5 C.CNTINLE

HPITE $(K, T)$

7 FCFHATIIHO,12H URTER ACUNAT)

Q

r,o Tत 4

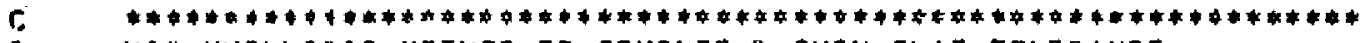
C lisf MUEILEDIS MFTHCT TO COMPUTE R SUCH THAT TOLERANCE
C NN LRAR IS SATISFIEC. MUEI.LER RJUTINE CALLS SLARCUTINE F TO
r. CCMDITT LQLQ = F(F) - LI
c. - 4 की

3 CALL DTNI(D),LN,F,PNH], RN, 20, JE-5, 6C,IER)

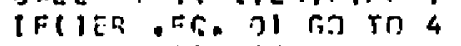

HRITE(':A'I) IER

11 FRQWATIIH1,14HNIIELLER FAILED. I5I

$R !=0$

$4 \quad R 2=R ! 111 .-P C P J 1$

$\mathrm{L} 3=\mathrm{XI}_{\mathrm{I}} \mathrm{X} \times \mathrm{Z}$

$X P A R=\mid \times 1+\times 21 / 2.0$

$L 1=01 * T+1$

$L ?=P Z+T+2$

$A j=1 T H 2 * R ? * * 21 / 2,-i \times 2 * Y 21 / 2 *-L 3 * Y 0 *(T H I * R) * * 2 j / 2,0 *$

$1 \quad(X ?-A)+Y\}+|(X)-1|+\mid A-Y) \mid 1 / 2.0$

HRITFIt,FCCI $A+B, P C F J, Y C, L$

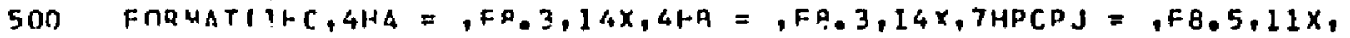

1 5HY0 $=, F P .3,13 \times, 4 \mathrm{HL}=, F B .31113$

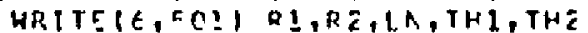

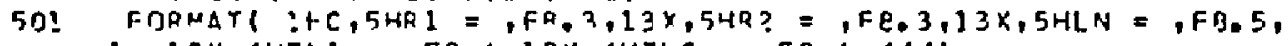

$1 \quad 13 Y,(H T F=, F Q, 4,12 X, 4 H T H z=, F 9,4 / 1 / 1$

URITR(S, SCZ) VI,YZ̃,LI,LZ

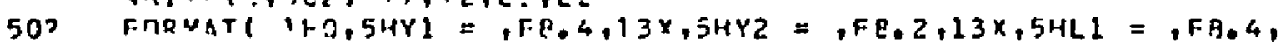

$11 ? \times, F H L 2=.59 .41111$

HPITE(E, ECE) YRAR,AJ,L?

503 FREMATI I $0,7 H \times R A F=, F E .4,11 \times, 5 H A J=, F 9.2,13 \times, 5 H L 3=, F 8.41$ ris in iOS

$c$
$c$
$c$
$c$
$r$

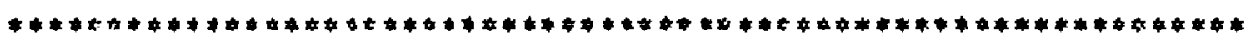

CCNDITICA XI LESS THAN A. CCMPIITE THE VILUE OF R SUCH THAT

$X i=$ THIS VALUE DF Q GIVES THE NAXTWLW VALIJE OF $L$

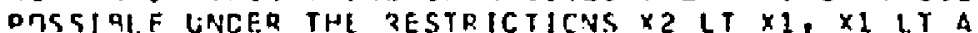

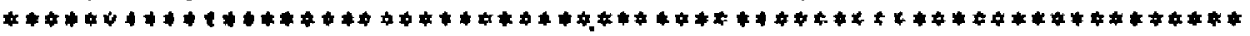

$\ln \quad \sin N=-1$

$P N=|A * 2+E * \#+Y O * Z-2 * 4 Y O B| /|Z *| Q-Y O \mid)$

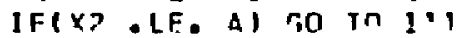

$S I R K=$ :

HRITETR, INE) I

505 ENRNATIIHC,1? OHCONDITICA X2 GT XI ANO XI LT A RCT SOLVED RY THIS

I PROGPAM. FITHER THFPE IS AC SMLUTICK OP A PETTER GUESS FOR RO

I IS OFGUIRFE I

C

C. ISE MIFLLERS YFTHCO TD COMPUTE FINAL LPDER ACUNS ON $a$

c. HHITH IS TRE CFMITITM THAT L3 $3=0.1$ NUELLER POITINE 
FTN

FINTSTICN FIQI)

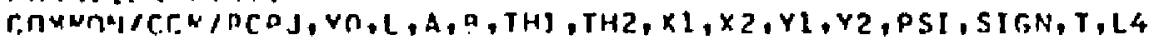

QF IL I, I.

तATA OI $1=, 14259271$

C $\quad$ C

C IF OTRI IS SIJPA THAT L-HAR hILL TE COMPLEX, THE VALUE

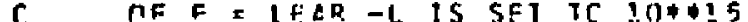

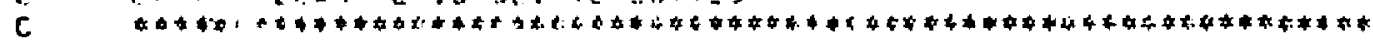

$\therefore \quad Q ?=0: 111.0-D C P J 1$

$A i=-V Q+47-\bar{x} \cdot n+P 2 * Y G$

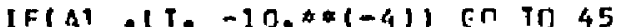

[rial: $[1 ; 0, i n]=0$.

$x ?=\operatorname{sop}(A \mid A)$

$r z=00+40$

$T 42=1 T M(X 2 / Y 2)$

IFIY? . FC. O.I TH2 = PI/2.

IFI THZ LT. C. I THZ $=\mathrm{TH}+\mathrm{TI}$

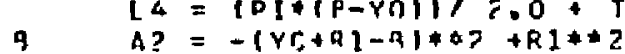

IFAA? $17,-10, * *(-4))$ Gn TO 50

$I F I A\}+t T \cdot C . I A Z=C$.

IFI $A$ ? LT. C.I SE TO 50

$X 1=A+S I C A$ SORT(A2).

$Y 1=01+Y C$

$T=(D-Y: 1,(A-X I)$

$P S I=\operatorname{TIR}(T)$

IFI $A-x \mid$, CE. 0.1 , TO TO 10

PSI $=P S_{1}+P_{1}$

in IFIA - XI .EQ. O.) DSI = PI/Z.

$T H]=D[/ 2 .+D S I$

$F=T H] * P)+T H 2 * 02+A(4 S(\times 1-\times 2)-L$

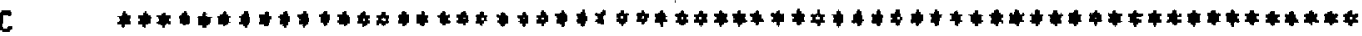

C IF VALIJE CF VARIAPLES CA EACH ITERATICN IS DESIRED, REMOVE

C C TA FCLLCWING NRITE STATENERT.

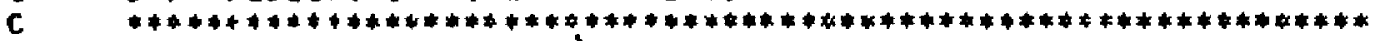

C HRITFIS,EII RI,THI, XI,A, X2,L4,F

51 FORMATIICH FIINCTION F R1,FB, 4, 5X, 3HTH1,FE,4,5X,2HX1,F0,4,5X,

1 LHA,FA, 4, 5X, ?.HX2,FR.4,5X,2H,4,FA, 4,5X,1HF,FB, 41 RETURN

$45 \quad F=10.0 * 15$

WRITE $(6,1) ?)$

103 FORMATIIHC,12HCOMPLEX IA F I RETURN

$50 \quad F=10.0+115$

WRITE $(6,102)$

RETIJRN

END 
DI GARN

- EFN

SIURCE STATEMENT

IF

C CALLS SURPCUTINE O.

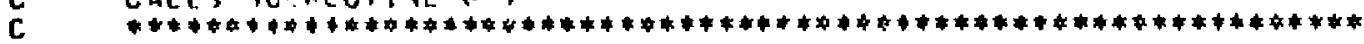

11 CALL RTNII RI,LN,G,RNPJ, RN, IC,CE-6,20CO,IERI

IFI 1ER EO. OI GR TO $20 \mathrm{~L}$

WRITE $\{6,12\}$ IER

D I 4

Sח $\operatorname{TH}^{2}=\mathrm{RI}$

END. 
GTN

- efN - SnURCE STATEkENT - IFN(S) -

FUNCTICN G(R!)

COMHON/CCH/PCPJ, YO,L, A, R, THI,THZ, X1,XZ, YL, Y2, PSI, SIGN, T, L4

REAL L, 1.4

DATA DI 13.14159271

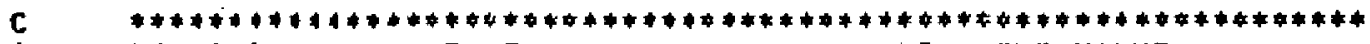

C IF R(K) IS SIJCH THAT L-RAR HILL BE COMPLEX, THE VALUE

C IS SFT TR 10\#15

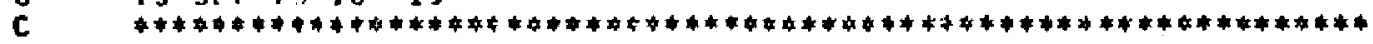

$\therefore 2=R ! 1(1.0-$ PCP.J)

$A L=-Y O * 42-2,0 * R ? * Y 0$

IF (AI $[T *-1 C * *(-4))<0$ TO 45

If (A) - I T. 0. I AI =

$X_{2}=$ SORTIAI)

$Y 2=R 2+Y O$

$T H 2=A T A K(\times 2 / Y 2)$

IFIYZ. FQ. COI THZ $=$ PI/Z.

IFI TH2 - t 1. n. I THZ = THZ + OI

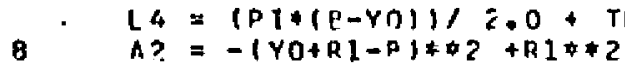

IF $\{A 2 \cdot$ : T. $-10 . * *(-4)\}$ GO TO 50

IFIA2 LT. O. I AL = 0 .

$X I=A+\operatorname{SICN} * \operatorname{SOPT}(A P)$

$Y I=R I+Y O$

$T=(P-Y \mid),(A-X \mid)$

PSI $=A T A M(T)$

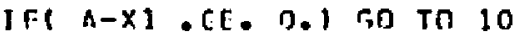

$P S I=P S I+P I$

ID IFIA - XI.EC. O.I PSI $=$ PI/Z.

$T H 1=P I / Z_{0}+P S I$

$G=X I-x ?-10 *(-2)$

c

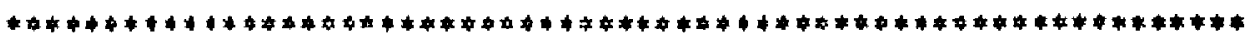

IF VALIIF CF VARIAPLES CA FACF ITERATICN IS DESIRED, REMOVE

C CA FCLLCHING WRITE STATEMENT.

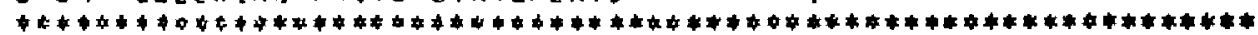

C HRITE $(6,5], 01, T H 1, \times 1,0, \times 2,1,4, r_{3}$

FORYATIISH FIJNCTICN , RI, FA, $4,5 X, 3 H T H I, F G, 4,5 X, 2 H X 1, F B, 4,5 X$,

$12 \mathrm{HA}, \mathrm{FB}, 4,5 \mathrm{X}, 2 \mathrm{HX}, \mathrm{FR}, 4,5 \mathrm{X}, 2 \mathrm{HL} 4, \mathrm{FB}, 4,5 \mathrm{X}, 1 \mathrm{HG}, \mathrm{F}, 4,41$ RETUTN

$45 \quad F=! 0.0 * 15$

HQT TE $(A, 104)$

104 FORHATIIFC,12HCRNPI.EX ING I RETUP V

$50 \quad F=10.0015$

HRJTE $(6,104)$

RETUPN

END

2

$\theta$ 
Appendix III

\section{ELASTIC FREE TRUNK SHAPE}

The computer program described in this appendix computes the cross-sectional shape for a free elastic trunk. The elasticity may be non-linear. The logic is similar to that presented in Section 4.4.

The initial input variables are:

a $\quad=x$ coordinate of upper trunk attachment point, $\mathrm{ft}$.

b $\quad=y$ coordinate of upper trunk attachment point, $\mathrm{ft}$.

$\mathrm{p}_{\mathrm{c}} / \mathrm{p}_{\mathrm{j}} \quad=$ ratio of cushion pressure to trunk pressure

$P_{j} \quad=$ trunk pressure $\mathrm{lb} / \mathrm{ft}^{2}$ absolute

$\ell=$ trunk length of $\epsilon=0, \mathrm{ft}$.

The elasticity of the trunk is defined by 15 points or less from the tension versus strain curve and the derivatives of the end points.

The input variables for the elastic curve are:

NN = number of points selected from the elastic curve (15 points maximum)

$A R G=$ value of tension $\left(R^{*} P_{j}\right)$ at each point, $l b / f t$.

$\mathrm{TAB}=$ value of strain (epsilon) at each point point $\mathrm{ft} / \mathrm{ft}$ or in./in.

DV (1) = the reciprocal of the derivative of elastic curve at left end point, $\mathrm{ft} / \mathrm{lb}$

DV (2) = the reciprocal of the derivative of elastic curve at right end point, $\mathrm{ft} / \mathrm{lb}$

This program is similar to the program described in Appendix I, except the equation to be satisfied in this case is as follows: 


$$
F\left(R_{1}\right)=\bar{\ell}-\ell(1+c)=0
$$

where $\epsilon=f_{1}\left(R_{1}\right)$ and $\bar{l}=f_{2}\left(R_{1}\right)$

The program uses the following subroutines:

Function $F\left(R_{1}\right)$ evaluates $\bar{l}-\ell(1+c)=F\left(R_{1}\right)$

Function $D F\left(R_{1}\right)$ evaluates the derivative of $F\left(R_{1}\right)$.

Subroutine RTM1 uses Mueller's Iteration Method to converge on the solution of $F\left(R_{1}\right)=0$, once the solution is bounded. This subroutine is listed in Appendix $I$ and is zot repeated here.

Subroutine SPLN1 develops the coefficients for a third degree interpolating polynomial between each pair of points which specify the clastic curve. These coefficients are stored in the $\mathrm{C}$ matrix whose dimension is 4 (INN - 1).

Subroutine SPLN2 uses the coefficients developed by SPLN1 to interpolate for the value of at $R_{1} \times P_{j}$. The output of SPLN2 is a five dimensional vector $V$ with the following values:

$$
\begin{aligned}
V(1) & =\text { tension or }\left(R_{1} \times P_{j}\right) \\
V(2) & =\epsilon \\
V(3) & =\epsilon^{\prime} \\
V(4) \quad & =\epsilon^{\prime \prime} \\
V(5) \quad=\text { key; } \quad 1 & =\text { value of } V(1) \text { below the table } \\
& 2=\text { value of } V(1) \text { in the table } \\
3 & =\text { value of } V(1) \text { above the table }
\end{aligned}
$$


These values may be printed out by removing the comment notation from the write statement above statement 409.

The output gives the values of all input variables, the initial guess for $R_{1}$, the notation Mueller if the RTM1 subroutine was called, and the final values of the following variables:

$\dot{\ell}, \dot{T}_{t}, \epsilon, R_{1}, R_{2}, x_{o}, y_{0}, y_{1}, y_{2}, \phi_{1}, \phi_{2}$, and $A_{j}$ 
CIGGES

EDTRSF - EFH SOURCE STATEMENT - IFNISI -

CDMMOV / MAINF / NN, ARG, TAB, C, PJ, V

CDMMTN ICCI A, B,PCPJ,L

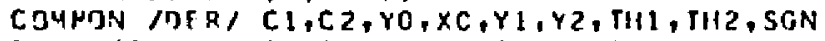

DIUFNSICV TAB (15), AHG(15), H(99), C (95), DV(2), V(5)

P.FAL LI,L?,L,LE,LA,LNMI

DATA PI $f \geq .1415927$

EXTEPANAL F

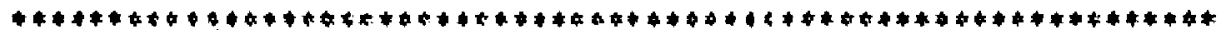

TOL IS A RELATIVE TCLERANCF GN LHAR . CAV RE CHANGEG BY

INSERTINS CART.

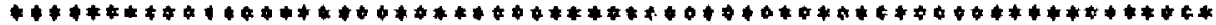

$\mathrm{TnL}=3 . \mathrm{E}-\mathrm{5}$

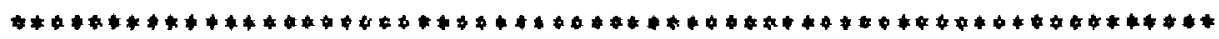

QEAD DATA FCQ TARLE DEFIVING FPSILGA AS A FUNCTION GF RAPJ AND

PASS TO SPLIVF INTERPELATICN SLBRDLIINE. CVIII ARD DV(?) ARE

THE DEHIVATIVES AT THF LEFT AND RIGHT FELICLIS RESPECTIVELY.

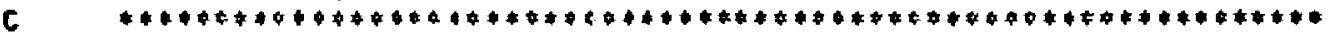

RFAC $(5, t 2)$ AN

FOHNATI:10)

REAC (5,3) DV

AEAC $(5,31)$ (ARG(1), I = 1, NH)

QEAC $\left.(5,31)(T A B \mid 1), J=1, N N_{1}\right)$

31 FORMATI BE 10.31

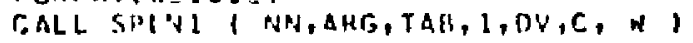

REA[ $(s+1]) A, B, \operatorname{PCPJ}, t, P]$

FORPATIST $1.4 \cdot 15)$

HRITI 16,400 ,

400

FORMAT I IHI, 40X, 38H*0***

ELASTIC [O. TD. SHAPE $\# \neq * *$

WRITE $(6,401)$

401 FORHAT I IHO, 13I, IHE,

HRITF I $5,40 ?$, CV

402 FORMAT 1 1HO, $5 x, 37 H+\%$ DERIVATIVES AT THE ENO POINTS,

1 19R.OF THE TAELE \#4

$2 / 14 x$, ELCARGI $=$, EP7.5, $10 X$, EHCARGN $=, E 20.51 / 1 / 1$

HRITF $5,4 C 3$, VA, I TABIII, ARGIII, I=I,AN;

403 FORNAT I IHO, 5X, 14HTAELE FRTRIES, I2,

$1 / 1,5 x, 2 F 20.511$

HQITF $(0,4 C 4) n$, B, PCPJ, L, PJ

404 FCRMAT I I C, $5 X_{1}, 4 H A=$, EZC.5, $20 X, 4 \mathrm{HA}=$, E20.5, $20 \mathrm{X}$,

1 THPCPJ $=$, EPO.5, $/ 6 X_{4} 4 \mathrm{HL}=, E 20.5,20 x, 5 H P J=, E 19.5$,

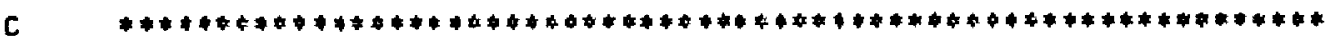

C FIX SIGN CA SCUARI ANC.T.

C

$5 \mathrm{GN}=1 . \mathrm{C}$

IFI PI*STRI $(A * 2+B * 2 I / 2 . \Pi$.LT.LI $\operatorname{SGN}=-1.0$ 
DI GGES

FOTPSH - FFN SCURCE STATEMENT - IFN(S) -

C. RO EOUALS INITIAL GUESS FOR RI. CAN HE CHANGFC BY INSERTING CARD.

C

RO $=\operatorname{SORT}(A * * ?+2 * 2) *(1.0+10 . * 4(-6) 1 / 2$.

HRITF

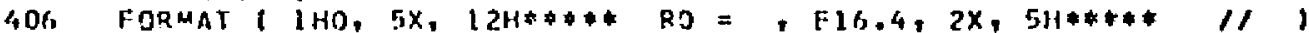

38

$R N=R D$

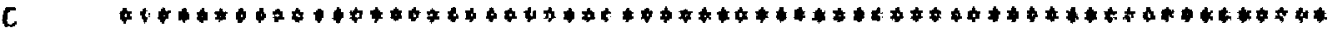

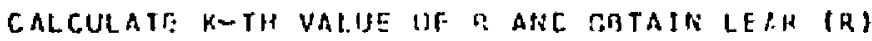

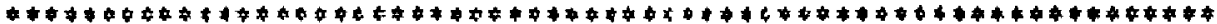

$74 \quad$ Dn $68 K=1,1000$

C $\quad$ क

SUIDOUUTINE I CCMPUTFS LIIAR - LIL + EPS)

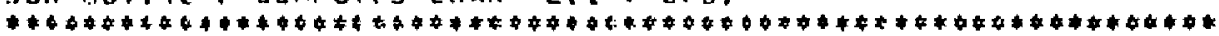

$2 \quad P L N=F(R N)$

$L N=P L N+L$

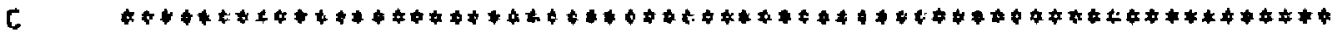

C IS R VFGATIVE OR IS LUAR (R) CJMPLEX. IF SO $R(K+1)=(R(K+1)+R(K)) / 2$

C (THIS CCCLIS WHET R(K) IS TCC SYALL)

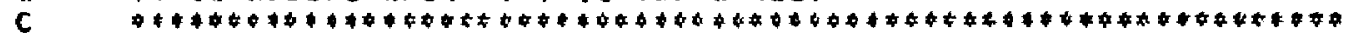

JF(PLN .VE, I $0 . * 15, A N D . R N, G T .0 .1$ GO TC 4

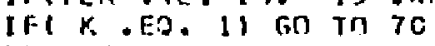

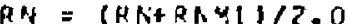

60 TO 2

4 IFIK.FO. 1$)$ G TO 5

C $\quad$ C

DETER'INE IF SOLUTICN HAS BEEN BDURDED. IF SC SET BDUNDS

ANO CALL NLELER POLIINE. IF NOT COFPLIE P(K+1) USING

NEHTDN"S FCFUIJLA.

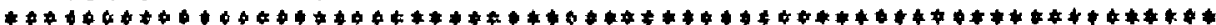

IFI SIGN( I, LL-LNI . VE. SIGNI1, L-LNHIIIGO TO 100

$5 \quad L N M 1=L N$

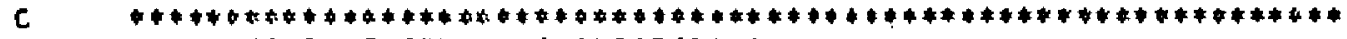

C SUBRTUTINF EF CCMPUTFS ILGARIRI):

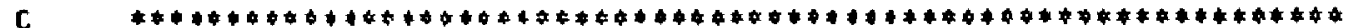

$D L N=-D F(R N)$

62

$R \mathbf{L}=\mathrm{RN}$

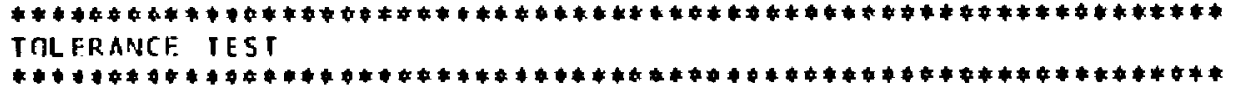

IFI ABS (LH-L) .LT, TOL*ABSILI) GO TC 110

RNMI = RN 
ovi

I UL US

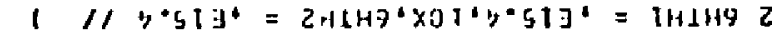

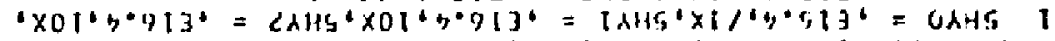

18

OB

Bl

$7 L$

EL

69

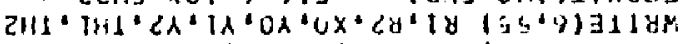

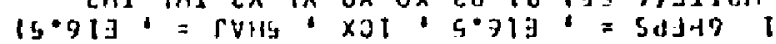

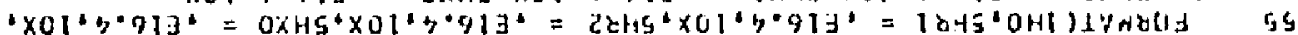

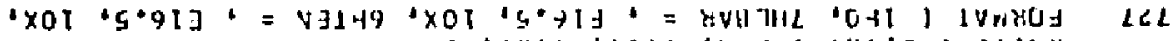

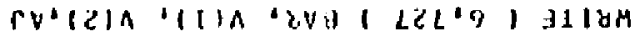

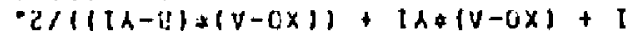

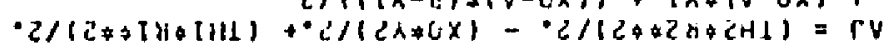
$28 * 211+1001 H L=649$ $V / 0 x=20 x$

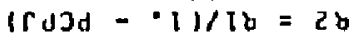
4I

dLIS

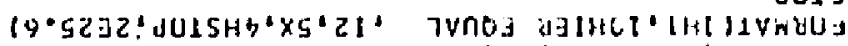
N7'ly'y (3)

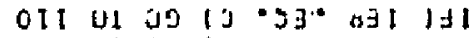

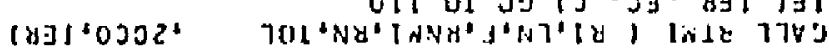

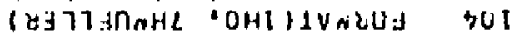
(>0I.0) IIIIA SoI Nlli $=1$ WV Iw $1 \mathrm{~h}=\mathrm{Nu}$ $\mathrm{N} \mathrm{di}=\mathrm{hnc}$

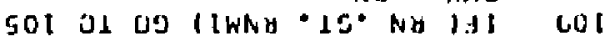

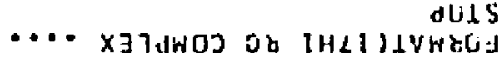
12 JIINIINOJ $N 70 /(7-V))-N$ I $=N y$ 501 (1Lig) 11 lim

$0 L$

$02 / 80 / 20$

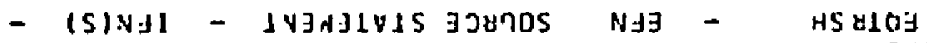
SH9פIU 
DIGGFS

FTN - EFN SCURCE STATENENT - IFN(S) -

$02 / 09 / 70$

FUNCTIJN F (PI)

CCAMDN, I RAINF, NN, APG, TAH, C, PJ, V

CEMUCS ITEA $A, Q, P C P J, L$

CCYNON IOEH, CI,C?,YC,XC,Y1,Y2,THI,THZ,SGH, PJDEPS

DIMENSICN C(99), ARG(15), TAB (15), V(5)

DATA PI/Z.14159??

REAL L

$c$
$c$
$c$

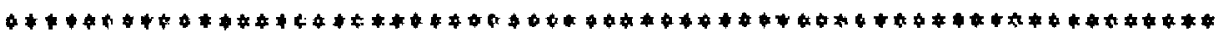

If R(K) IS SUC,I HHAT L-BSAR HILL BE CCHPLLX, II.E VALUE

DF $F=L H A R-L$ IS SET ID $10 * 15$

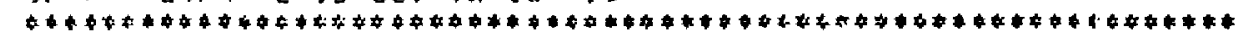

-

C.

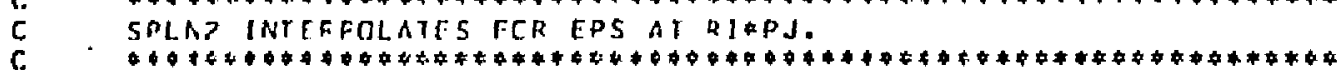

$V(1)=R 1+P J$

CALL SPLV? I MA, MZG. TAB, C, V,

C

C IF VALUF CF VARIAMLES CN EACH ITERATICA IS OESIRED. REMQVE

C. C ON THE THC WRITE STATEMENTS.

c 640 क

C. WRITE $T+409$, V, RI, PJ

Ang FORHAT I IHC, $\mathrm{jX}, ? 7 \mathrm{H}, \ldots .$. SPLIVE OLTPUT ....

$1 / 1(5525.7))$

EPS $=V(2)$

DEPS $=V(3)$

DJBEPS = PJ CEPS

R? $=\mathrm{R} 1 .,(1 . \mathrm{C}-\mathrm{PCPJ})$

$\mathrm{C} 1=(\mathrm{P} 1-[\mathrm{R}-\mathrm{A} 2), \mathrm{A}$

$C 2=4 / 2.0+(0 * 2) /(2.0 * A)-(4) * B) / A$

$150=(3.0 * 22+7.0 * C 1 * C ?) * 7-(4.0 * C 2 * 2) *(C 1 * 2 * 2+1.0)$

IFI ASO.LI. T.0) 6. TO 25

$50=\operatorname{SQPT}(A \mathrm{SO})$

$Y_{0}=(-2.0 *(02+C .1 * C ?)+5 G N \neq S 6) /\left(2.0 *\left(C_{1} * 2 * 2+1.0\right)\right)$

$x=C L A Y C+C ?$

$Y 1=Y n+41$

$Y ?=Y O+Z ?$

$\mathrm{TH2}=\operatorname{ATM}(X 0 / Y 2)$

IFI Y2,FC. O.I THZ=PI/Z.C

IFITHZ,LT. O.OT THZ = TH2 + PI

$P S 1=A T A N(1)-Y 11 /(A-X 0))$

If $A-X C) 2 C, ? 7,21$

20 $\quad$ DSI $=P S I+P I$

2. $T H I=P S I+P I / 2.0$

$F=R 1$ FHI $+22+\{H z-(* 11.0+E P S)$

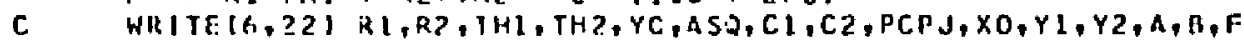


DIGSFS - EFN SOURCE STATEMFNT - IFNIS) -

FTN

RETUPW

$27 \quad P S I=p ! / 2.0$

GD in ? 1

$35 \quad F=17.0 * 15$

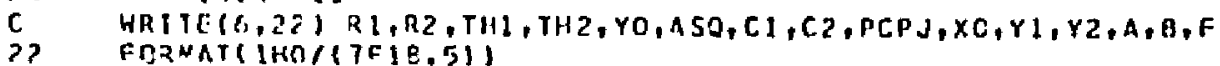

RFFURN

ENO 
DIGGSS DFRF

FUNCTION TFIR I)

CCMMTN TIER/ CI,C2,YO,XC,YI,Y?,TH1,TH2,SGR, PJDEPS

r.OKRTIN /CC/ A,B,PCPJ,L

REAL $\mathrm{K}, \mathrm{L}$

$K=1.0-F C P J$

$D C 1=(k-1.0),(k * A)$

$n C_{2}=-p / n$

$X=R 1 / K+C 1 * C$ ?

$Y=C, 1 * ?, 1.0$

$\left.\mathrm{DX}=1.0 / \mathrm{K}+\mathrm{Cl}_{1} * 1 \mathrm{C} 2 \mathrm{C}+\mathrm{C}_{2} * 1\right) \mathrm{C} 1$

$D Y=2.04 C 1 \% \mathrm{DCl}$

$Z=-$ SGN $*$ SQR I $X * 2-Y *[2 * 2]$

$D T=(1.0)(2.0 * 2)) * 12 .(* x+0 x-(2.0 * Y * C 2 * D C 2+C 2 * 2 * D Y) 1$

DYO $=(1.0 / Y * 2) *(-Y *(D X+D Z)+(X+Z) \neq D Y)$

$D \times O=C 1+C Y C+Y O+D C 1+D C 2$

$D Y 1=D Y D+1.0$

DY2 $=0 Y 7+1.0 / K$

$S=n-Y 1$

$T=A-x n$

DST $=(1.0 / T * 2) *(-T *[. Y)+5 * 0 \times 0)$

DXOYZ $=(1 . C / Y Z * 2) *(Y 2 * 0 \times C-X 0 * 0 Y 2)$

DPSI $=$ DST $/(1) 0 *\{5 /\}) \div 2\}$

DTH $=\operatorname{RXCYZ} /(1.0+(X 0 / Y Z) * \geqslant ?)$

DTHI $=\operatorname{CPSI}$

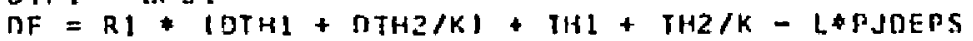

RETURN

END 
CIGGFS

SPL. I

- EFN

SOURCE STATEMENT

IFN(S)

$02 / 09 / 70$

SUBRTUTINE SPLAL (N, X,Y,J,D,C,H) SPLNL

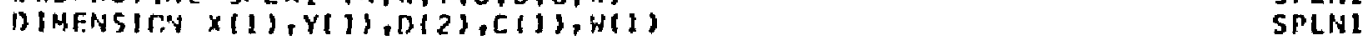

C

C OVER THE INTERVAL X(I) TO X(IAl). THF INTERPOLATING SPLHI

C.

PCLYNCMIAL $Y=Y(I)+\Lambda(t) * Z+B(1)+Z * 2+E(1) * Z * 3$

Hhe LE $z=(x-x(1))(1 \times()+1)-x(1))$

SPINI

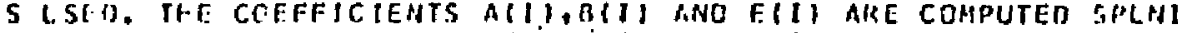

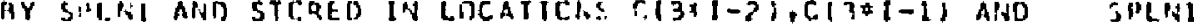

C (3⿻ I) HESPIECTIVELY.

HIJIF HERKING IA THE ITH INTERVAL,THE VARTABLE O HILL SPLNI

REPRESENI $C=X(1+1)$ - XII). ANO YII) WILL REPRESENT SPLNI

$Y(1+1)-Y(1)$

SPLNI

-

$0=x(3)-x(1)$

$Y I=Y(\geq)=Y(1)$

SPLIH

SPLN1

SPLNI

IF (J.RO.z) Gn in 100

SPLHL

$c$
$c$
$c$

IF THE FIRST DERIVATIVE AT THE END POINTS IS GIVEN,

SPLNI

A 1 I IS KIVGN, ANO THE SECOHD EQUATIOA BECOMES SPLH1

NFPFLY $A(I)+E(1)=Y I-Q \circ D(1)$.

SPLiN1

$(1)=7+0(1)$

$C(?)=1.0$

$W(Z)=Y 1-C, 11)$

ro $\operatorname{th} 2 \mathrm{~m}$

SPL NI

SPLIHI

SPL.N1

SPLN1

SPLN1

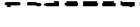

IT THE SECCNJ LERIVAIIVE AT TIE TND PCINTS IS GIVEN

HIII IS KNIHN, THE SECDND ECUATIOS BECCMES

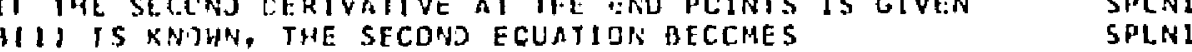

A(1)+F(1) $=Y 1-C .5 * D * Q * D(1)$. DURING THF SILUTION OF SPLHI

THE 3H-4 EOLATICINS, AI WILL BE KEPT IA. CELL CIDI SPLNI

IASTEAD OF CII TO RETAIH THE TRIDIAGCMAL FCHM OF THE SPLNI

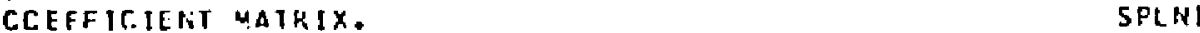

С

ino $C(?)=0.0$
SPLNI

$H(2)=0.54 C .2 * \Gamma, 11) \quad$ SPL.N)

$\begin{array}{ll}2 n n \quad M=N-2 & \text { SPLNI }\end{array}$

IFIN.LE.D) EO TO $350 \quad$ SPLNI

C

C UPPEQ TRIAIGULARTZAIION JF THE TRILIAGCNAL SYSTEM OF SPLNI

C ECLATIENS FDR THE COEFFICIENT HAIRIX FOLLOHS--

C.

on $303 \quad I=1, \mu$

$A I=C$

$D=x(1+2)-x(1+1)$

$H=A / / 7$

C. $(3 * 1)=-H /(\bar{c}, 0-C(3 * I-1))$

$W(30 I)=(-Y[-H(3) 1-1) / 12.0-C(3 * \mid-1))$

$\mathrm{C}(3+1+1)=-1+\mathrm{H} /(\mathrm{H}-\mathrm{C}(3+1))$

$H(3 * 1+1)=(Y I-H(3 * 1), / H-C(3 * 1))$

$Y 1=Y(1+2)-Y(1+1)$

$C(3 * 1+2)=1.0(1) .0-(3 * 1+1))$

. 33n $H(3 * 1+2)=(Y)-4(3 * 1+1)(1)(1.0-(13 * 1+1))$

SPLNI

SPLNI

SPLN1

SPLNI

SPLN1

SPLNI

SPLNI

SPLNI

SPLNL

SPLNI

$c$

SPLN 


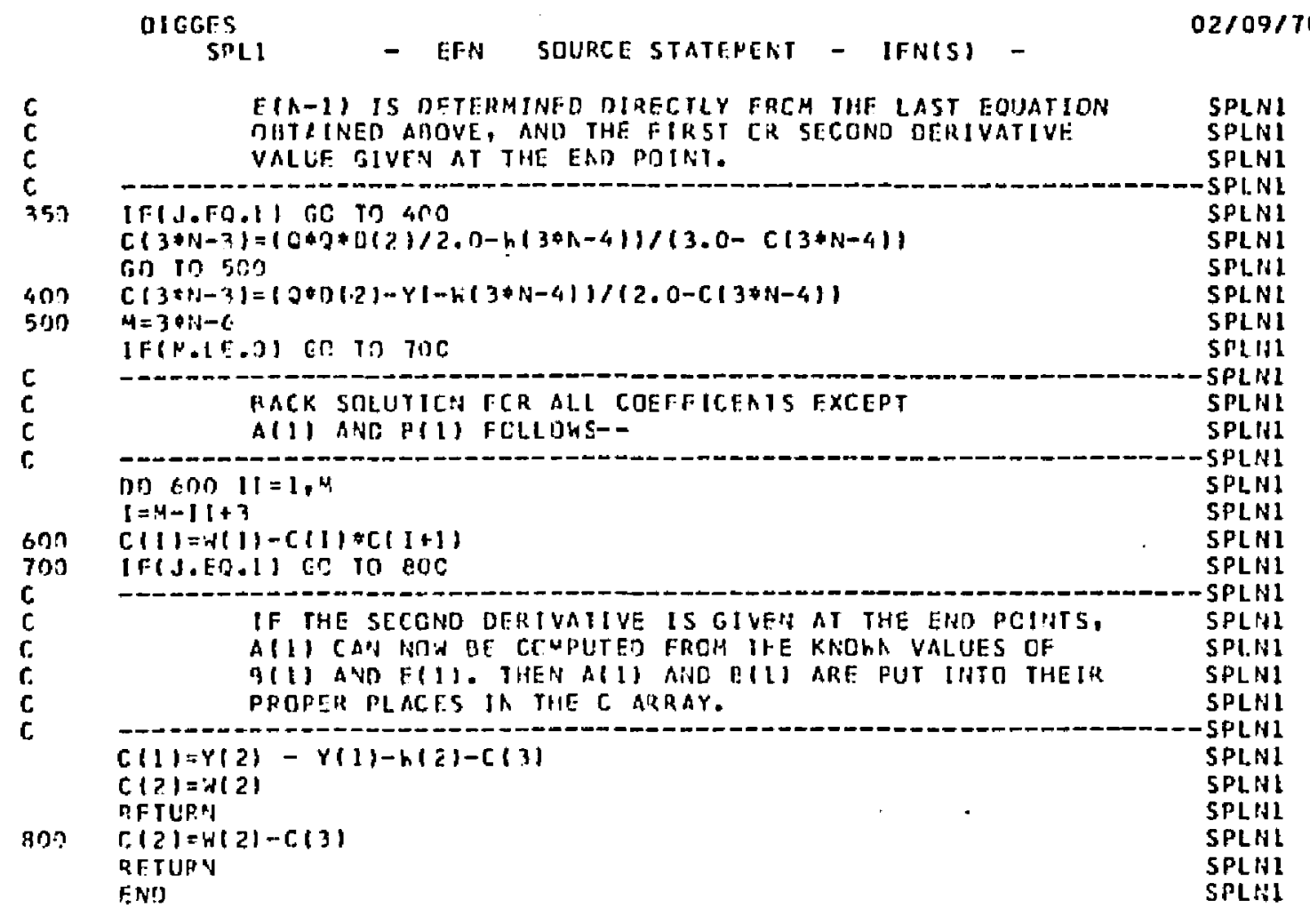




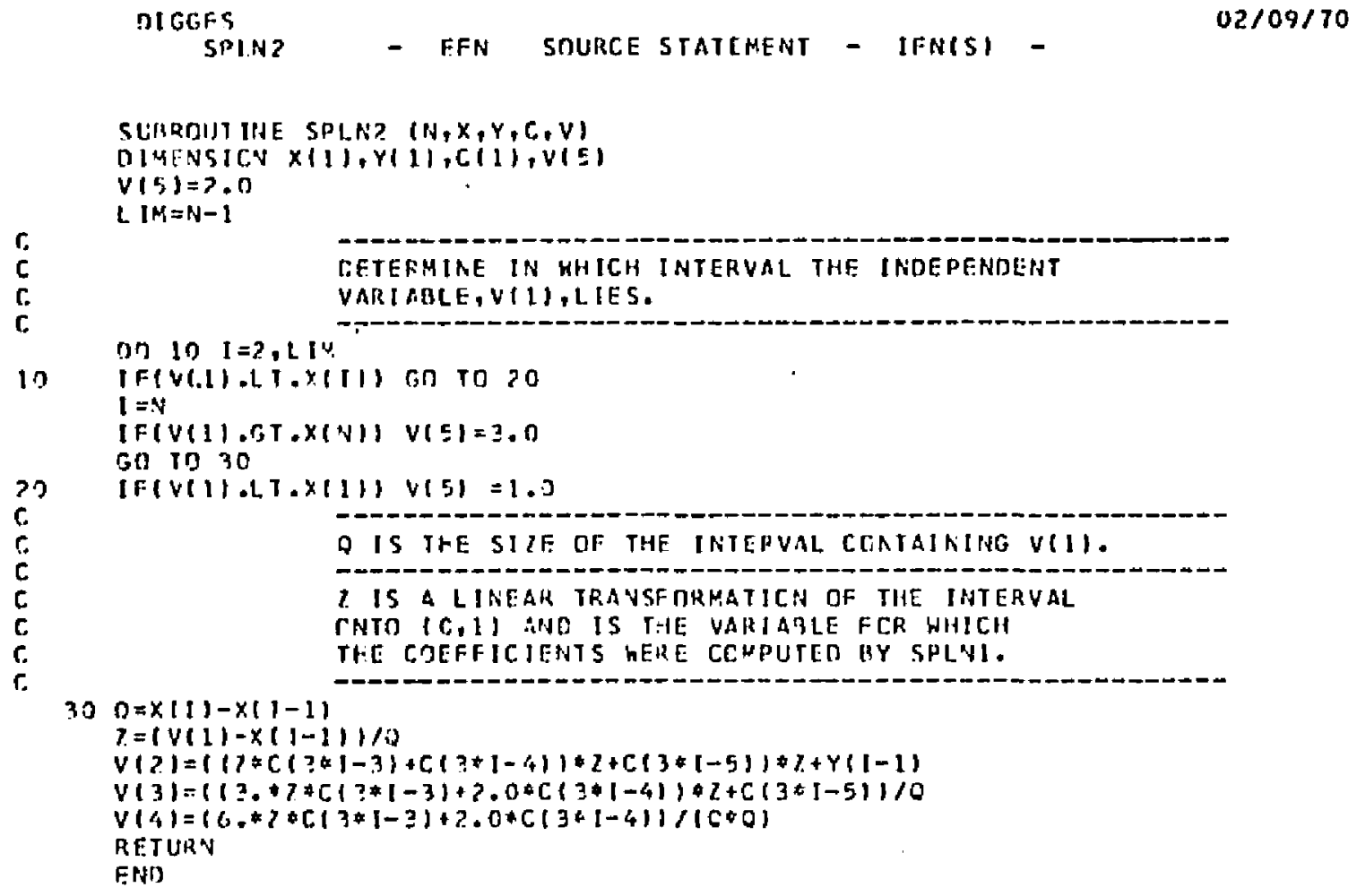




\section{Appendix IV}

\section{TRUNK CONSTRUCTION}

The trunk section was made of a nylon-hypalon material. The dimensions of the piece of material, before fabrication of the trunk, were 59-1/2 inches by 33 inches. Approximately four inches of the length was used for attaching the trunk to the model structure; the unpressurized length of the trunk became 55-1/2 inches. One-half inch of the material was folded over and sewn along each edge of the trunk to increase the stiffness of the edge (see Figure IV-1). A strip of trunk material 1-1/2 inches wide was sewn along either edge of the trunk to act as a sealing flap. When the trunk was inflated, pressure inside the trunk pressed the flap against the walls, resulting in an effective seal. A nylon string inside the fold of the flap was used as a drawstring to slightly decrease the length of the free edge of the flap. The final width of the test specimen was 32 inches.

The trunk was perforated with 192 holes of $5 / 16$ inch diameter. The holes were arranged in 8 rows of 24 holes each, as shown in Figure IV-1. The centerline of the outside row of holes was located 31 inches from the outside attachment point. A 1/16 inch diameter hole was punched at each of the pressure tap locations indicated in Figure IV-1.

The pressure taps used to measure static pressure on the outside of the trunk are shown in Figure IV-2. A 2-inch length of 1/8 inch O.D. copper tubing was flared and flattened at one end to give a thin flat flange. The tubing was bent, as shown, and cemented to the trunk over the $1 / 16$ inch hole with a prepunched square piece of trunk material. Plastic tubing was connected to the copper tubing, and cemented to the trunk for a short distance. Thus, motion of the copjper tubing and a corresponding deflection of the trunk surface were prevented. The outside surface of the trunk had nothing protruding to clisrupt the flow, and the area in which the pressure was measured was a smooth continuation of the trunk contour. 


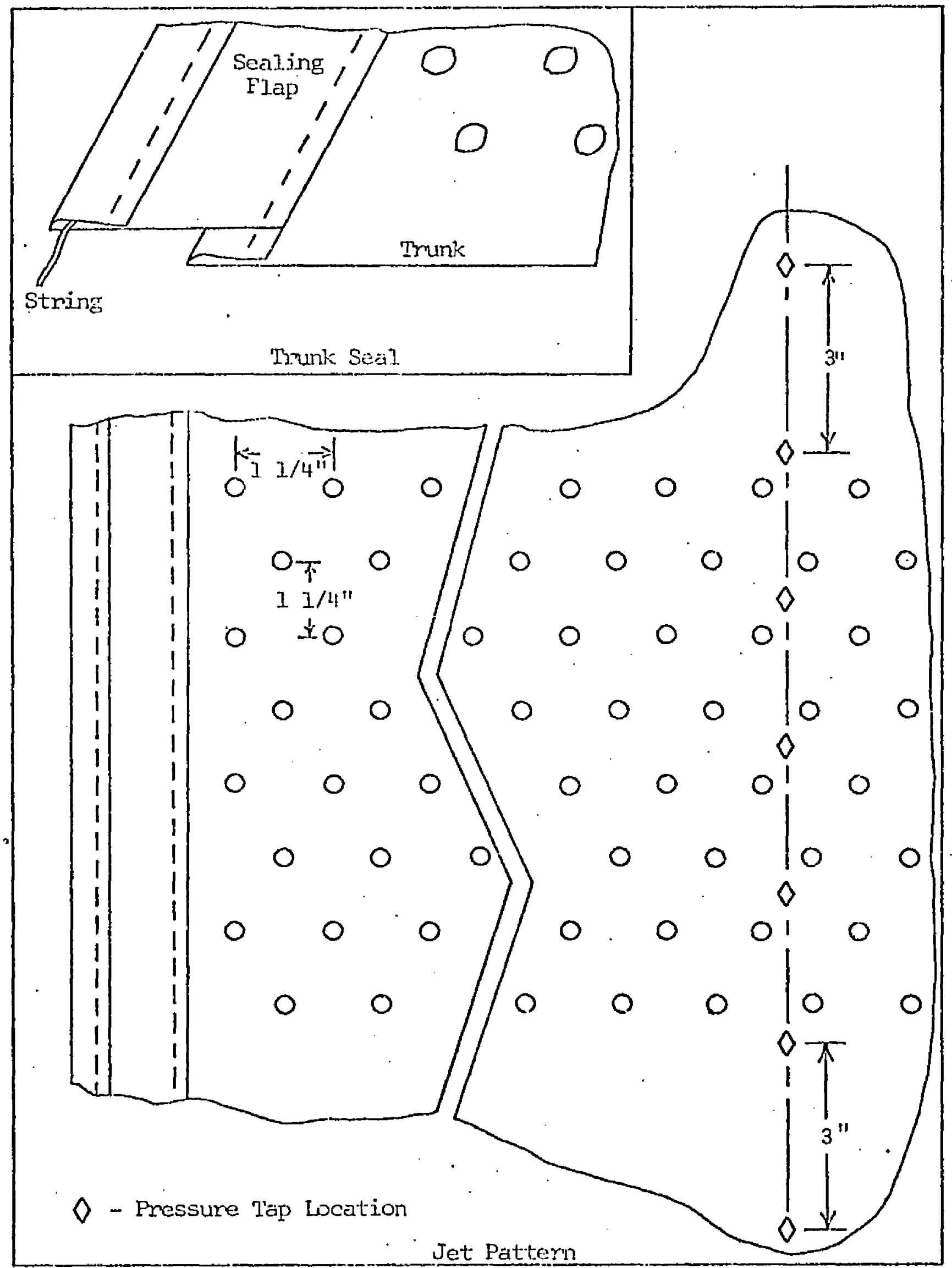

TRUNAK SPECIREEN DETAILS

FIGURE IV- 1 


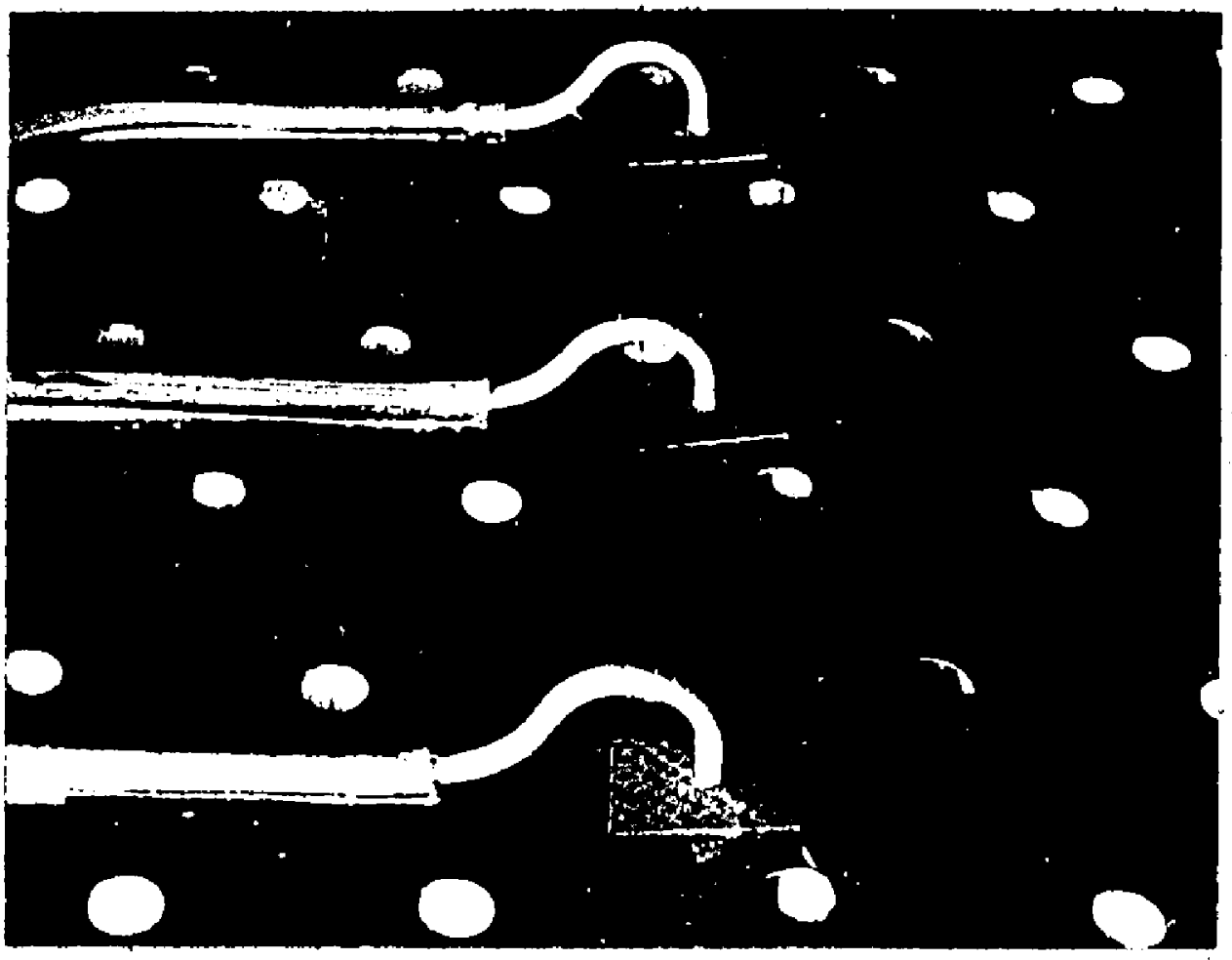

TRUNK. PRESSURE TAPS

FIGURE IV-2

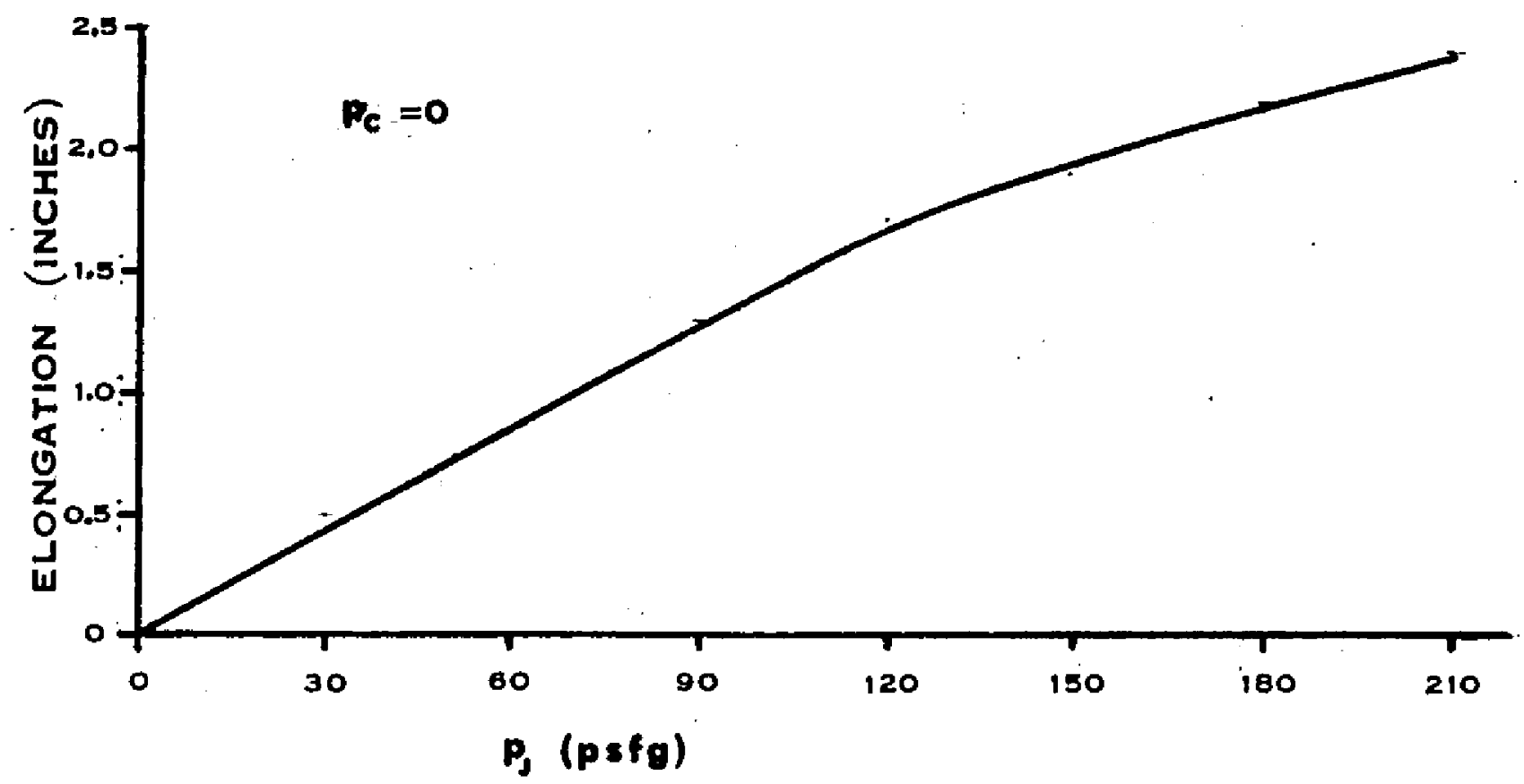

TRUNK MATERIAL ELONGATION

FIGURE IV-3 
A section of the trunk material was tested for tension-elongation in a tensile test machine. The results were used to predict the elongation of the trunk at various pressure levels. Figure IV-3 shows the resulting pressure-elongation curve for the trunk under test. Equation (4-1) was used to relate the tension to the trunk pressure. All results presented in Chapter 6 were corrected for trunk clongation. 


\section{Appendix $\mathrm{V}$ \\ DETERMINATION OF FLOW LEAKAGE}

The flow leakage in the model was measured as a function of trunk pressure to enable correcticrs to be made to subsequent flow calculations.

Before the holes had been punched in the trunk, the trunk was attached to the model and inflated. In this manner, a measuroment of the flow leakage between the trunk section and the walls of the model was made. A 1.2 inch orifice was used for flow measurement because the flow rate was quite low. The flow leakage measurements are presented in Figure $V-1$. 


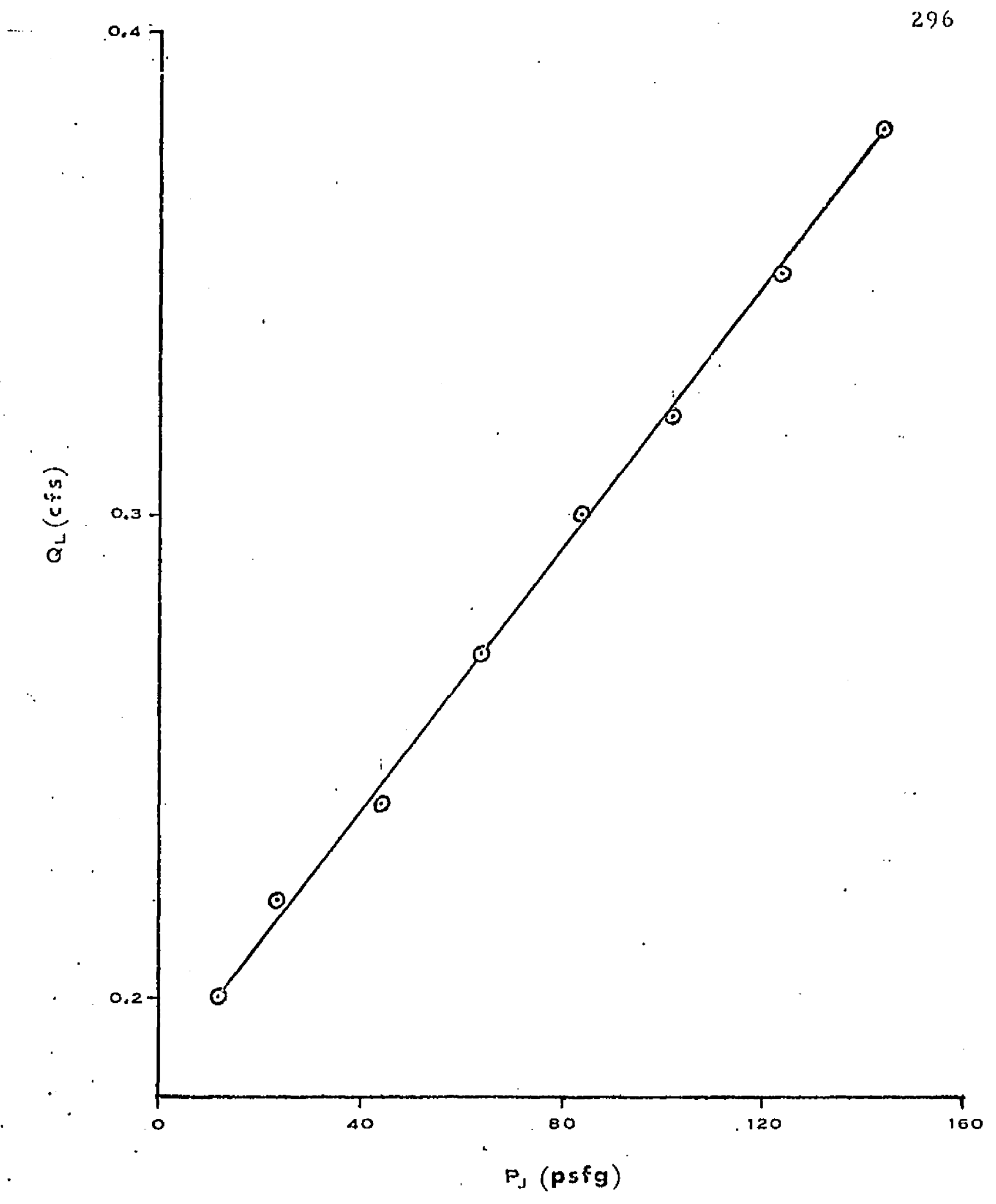

LEAKAGE VARIATION WITH TRUNK PRESSURE FIGURE $V-1$ 


\section{Appendix VI \\ COEFFICIENT OF DISCHARGE OF TRUNK}

The coefficient of discharge of the trunk $\left(C_{X}\right)$ is the flow coefficient for the entire orifice ara of the trunk $\left(a_{j}\right)$, in the abserice of cushion pressure.

With the movable floor removed from the model, the air gap between the trunk and the bottom of the model was sufficiently large that no restriction was presented to trunk flow. Thus, the pressure on the outside of the trunk was equal to atmospheric pressure. The system was operated throughout an extended range of trunk pressures, $10-140$ psf, and the data required for flow calculations were recorded.

The ideal rate of flow through the holes would be that predicted by a combination of the laws of conservation of energy and mass.

$$
\mathrm{o}_{\mathrm{id}}(\mathrm{cfs})=\sqrt{\frac{2 \mathrm{~g}_{\mathrm{o}}}{\gamma} \mathrm{p}_{\mathrm{j}}}\left(\mathrm{a}_{\mathrm{j}}\right)
$$

The coefficient of discharge of the trunk is herein defined as the ratio of actual jet flow, when there is no cushion present, to the ideal jet flow.

$$
c_{x}=\frac{a_{i}}{\sqrt{\frac{2 g_{o}}{\rho} p_{j} a_{j}}}
$$

To make the results applicable to subsequent runs when a cushion exists under part of the trunk, $C_{x}$ was plotted as a function of $P_{x} / P_{j} . P_{x}$ is defined as the average of the absolutc cushion pressure and atmospheric pressure. 


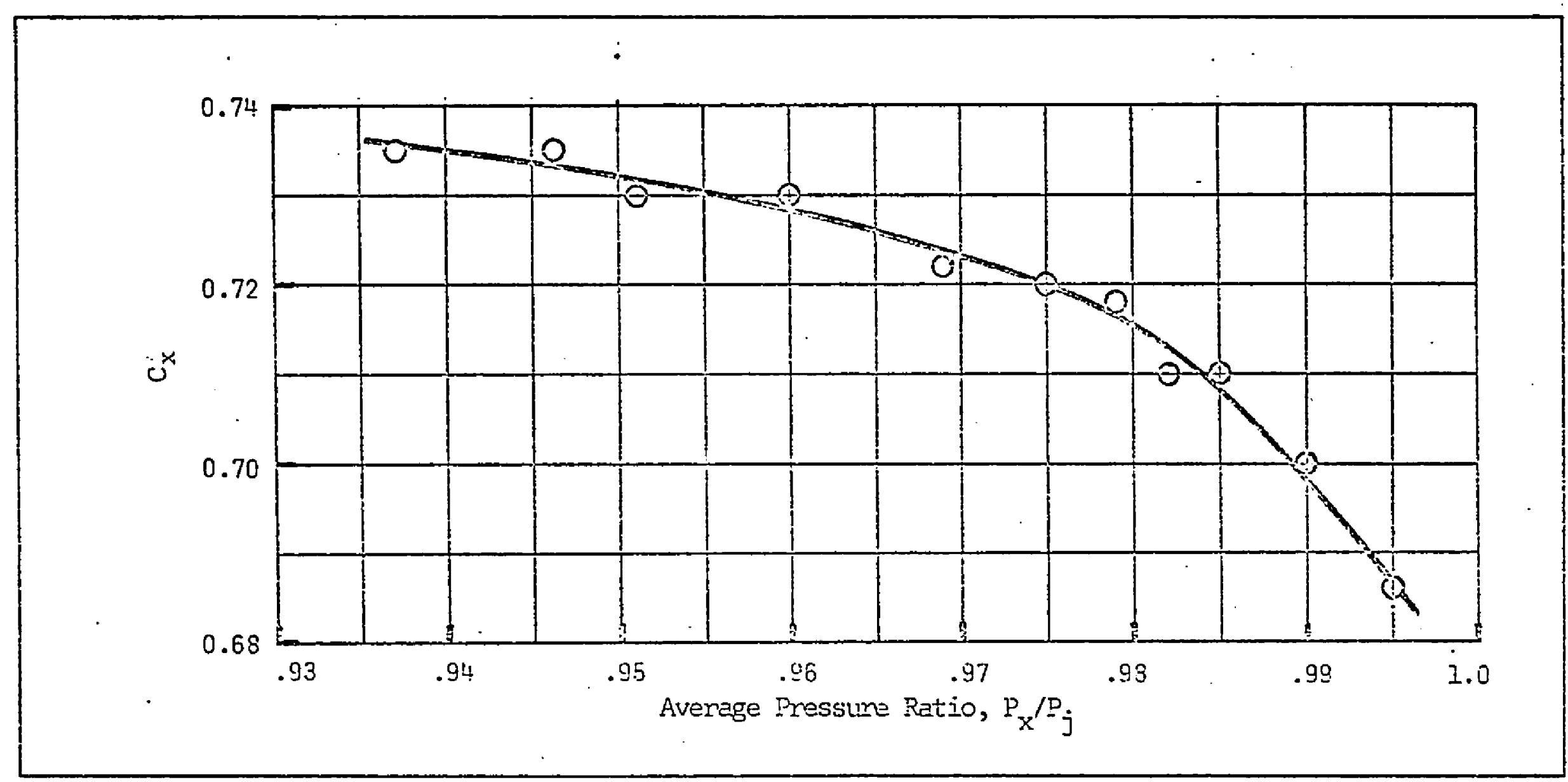

TRUNK DISCHARGE COEFFICIENT VS

AVERAGE PRESSURE RATIO

FIGURE VI-i 


\section{REFERENCES}

1. T. D. Earl, AFFDL-TR-67-32, "Air Cushion Landing Gear Feasibility Study," Bell Aerosystems Company, Mar 1967.

2. T. D. Earl and R. H. Cooper, AFFDL-TR-G3-124, "Air Cushion Landing Gear for Aircraft," Bell Aerosystems Company, August 1968.

3. C. Stauffer, AFFDL-TR-69-32, "Ground Flight Test Report of Air Cushion Landing Gear (LA.4)," Bell Aerosystems Company, Apr 1969.

4. D. J. Perez and H. K. Brewer, AFFDL-TM-69-11-FDFM, "Air Cushion Landing Gear Skid Brake Heat Transfer Study," AF Flight Dynamics Laboratory, Fob 1969.

5. K. H. Digges and D. J. Perez, "Air Cushion Landing System for STOL Aircraft," AF Flight Dynamics Laboratory, V/STQL Technology and Planning Conference, 25 Sep 1969.

6. D. J. Perez, "Implications of Air Cushion Landing System on STOL Aircraft," AF Flight Dynamics Laboratory, 1969.

7. J. Taylor, "All the World's Aircraft," McGraw-Hill Publishing Co., 1964.

8. A. Harting, "A Literature Survey on the Aerodynamics of Air Cushion Vehicles," AGARD Report No. 565, 1969.

9. T. D. Earl, "AGARDograph on Ground Effect Machines," AGARDograph No. 67, Jan 1962. 
10. J. C. M. Frost, "Flow Phenomena of the Focused Annular Jet, Symposium on Ground Effect Phenomena," Princeion University, Oct 1959.

11. J. C. M. Frost, "The Canadian Contribution to the Ground Cushion Story," Canadian Aeronautical Journal, October 1961.

12. R. K. Greif and W. H. Tolhurst, "Large Scale Wind Tunnel Tests of a Circular Planform Aircraft with a Peripheral Jet for Life, Thrust and Control," NASA TN D-1432, Feb 1963.

13. J. B. Chaplin, "The Development of a Multi-Cell Plenum Concept," AlAA Paper 64-188, May 1964.

14. W. Z. Stepniewski, "Performance Possibilities of Subsonic Airplanes Taking-Off and Landing on the Ground Cushion," Symposium on Ground Effect Phenomena, Princeton University, Oct 1959.

15. W. Z. Stepniewski, "The Subsonic VTOL and GETOL in Perspective," Aerospace Engineering, Oct 1959.

16. S. Montuoro, "Preliminary Results of an Experimental Investigation of Various Annular Jet Configurations in Ground Effect," Atti del $V^{e}$ Congresso Aeronautico Europeo, Venezia, Sep 1962.

17. W. Z. Stepniewski and F. J. McHigh, "Performance Considerations of Ground Effect Take-Off and Landing (GETOL) Transport Aircraft," New York Academy of Sciences, Vol 107, Article 1, Mar 1963. 
18. W. Z. Stepniewski, et al., "Research Program to Determine the Feasibility and Potential of the Ground Effect Take-Off and Landing (GETOL) Configuration," Vol 1, US Army Transport Research Command, TCREC Technical Report 62-63.

19. Convair's "GETOL Flight," Feb 1963.

20. W. D. Reeder and F. W. McDonald, "GETOL Aircraft, A Research Status Report," Institute of Aerospace Scienncs, IAS Paper 62-182, Jun 1962.

21. Ph. Poisson-Quinton, "Two-Dimensional Studies of a Ground Effect Platform," Symposium on Ground Effect Phenomena, Princeton University, Oct 1959.

22. Ph. Poisson-Quinton, "Influence of the Proximity of the Ground on the Aerodynamic Characteristics of V/STOL Aircraft Using Jets," AGARDograph 46, Symposium on V/STOL Aircraft, Part II, Jun 1960.

23. K. Dau, "Characteristics of a Rectangular Wing with a Peripheral Jet in Ground Effect," Part I, University of Toronto, Institute of Aerophysics, UTIA TN-56, Sep 1961.

24. J. M. Davis, "Characteristics of Rectangular Wing with a Peripheral Jet in Ground Effect," Part II, University of Toronto, Institute of Aerophysics, UTIA TN-59, May 1962.

25. K. Dau, et al., Aerodynamics of a Rectangular Wing with a Peripheral Jet for Air Cushion Take-Off and Landing," Canadian Aeronautics and Space Journal, Mar 1965. 
26. A. W. Carter and L. H. Person, "Investigation of the Free.Flight Characteristics and Handling Qualities of a Ground Effect Machine," NASA TN-D-3885, Apr 1967.

27. "New Developments in Air Cushion Vehicles," Hovering Craft and Hydrofoil.

28. R. McLeavy, "Jane's Surface Skimmer Systems," 1967-68 15t Edition Sampson Low, Marston, London, 1967.

29. R. McLeavy, "Jane's All The Worlds Surface Skimmer Systems," 69th Edition.

30. C. D. Bochler, "Aerodynamic Theory of the Annular Jet," Institute of Aerospace Sciences, IAS Report 59-77, Jan 1959.

31. H. R. Chaplin and B. Stephenson, "Preliminary Study of the Hovering Performance of Annular Jet Vehicles in Proximity to the Ground," David Taylor Model Basic, DTMB Aero Report 947, Sep 1958.

32. T. Strand, Inviscid, "Incompressible Flow Theory of Static, Peripheral Jets in Proximity to the Ground," Journal of the Aerospace Sciences, Jan 1961.

33. F. F. Ehrich, "The Curtain Jet," Journal of the Aerospace Sciences, Nov 1961.

34. M. J. Cohen, "Peripheral Jets in Proximity of the Ground," Journal of Applied Mechanics, Dec 1966.

35. T. P. Bligh, "The Potential Flow Solution of the Peripheral Jet of an Air Cushion Vehicle, Applicable to all H/t Ratios," University of Witwatersrand Report 30 , Dec 1965. 
36. J. T. Diez Roche, "The Preipheral Jet Theory," Proceedings of the 11 th International Congress of Applied Mechanics, Munich, Aug and Sep 1965.

37. H. H. Richardson and K. M. Captain, "Simplified Static Performance Characteristics of Low-Pressure Plenum and Peripheral Jet Fluid Suspensions," Massachusetts Institute of Technology, Jan 1968.

38. H. R. Chaplin, "Effect of Jet Mixing on the Annular Jet," David Taylor Mociel Basin, DEMB Aero Report 953, Feb 1959.

39. H. R. Chaplin, "Theory of the Annular Nozzle in Proximity to the Ground," David Taylor Model Basin, DTMB Aero Report 923, Jul 1957.

40. J. R. Stanton, "Some Design Problems of Hovercratt," Institute of Aerospace Sciences, IAS Paper 61-45, Jan 1961.

41. A. J. Burgess, "A Two-Dimensional Static Stability Theory for" an Air Cusion Vehicle with a Central Stability Jet," Department of Aeronautics and Astronautics, A.A.S.U. Report No. 256, University of Southampton, Great Britain, May, 1964.

42. V. Khanzhonkov, "Calculated Parameters of Air-Cushion Devices with Nozzle Device at Hovering Condition," Translation by Institute of Foreign Technology, WPAF Base, FTD-RT-23-1277-67, 15 Jan 1968.

43. T. Fujita, "Double Jet Theory," Working Paper No. 7, Vehicle Research Corp., Pasadena, Calif., 2 May 1962. 
44. Jack B. Esgar and W. C. Morgan, "Analyical Study of Soft Landings on: Gas-Filled Bags," NASA TR R-75, Mar 1960.

45. D. M. Gorman, Lt USAF, "Equilibrium Characteristics of a Distributed Ferịiiel al Jet," Thesis, AF Institute of Technology, School of Enginenring, W-P AFB, Ohio, Niar 1970.

46. S. D. Conte, "Elemeritary Numerical Analysis," Ne:Giav-Hill. 1965, p 65, ₹. 222.

47. 1. K. Spink, "Principles and Practices of Flow Meter Enginering," (Ninith Edition), Forboro, Massachusetts: Foroboro Compary, 1967.

48. ACME Research Committeo on Fluid Meters, "Fluid Meters, Their Theory and Application," (Fifth Edition), New York: Amirisan Socieiy of Mechanical Engineers, 1959.

49. i. S. Han, "Pressure Distribution in a Channel with Porous Walis," AF, DL Technical Report (To be published).

50. C. L. Stauffer, "Water Operations and Overland Braking Test Report oi Air Cushion Landing System (LA-4)," Technical Repert AFFDL-TR-69-125, Textron's Boll Aerosystems Co., Dec 1969. 